\title{
Proteomic Analysis of Lonicera japonica Thunb. Immature Flower Buds using Combinatorial Peptide Ligand Libraries and Polyethylene Glycol Fractionation
}

Wei Zhu ${ }^{1,2}$, Xiaobao Xu ${ }^{2}$, Jingkui Tian ${ }^{2}$, Lin Zhang ${ }^{2, ~ *}$, and Setsuko Komatsu ${ }^{1, *}$

${ }^{1}$ National Institute of Crop Science, National Agriculture and Food Research Organization, Tsukuba 305-8518, Japan

${ }^{2}$ College of Biomedical Engineering \& Instrument Science, Zhejiang University, Hangzhou 310027, China

\section{Supporting Information}

Supporting information includes supplemental figures and supplemental tables:

Supplemental Figure 1. Schematic workflow of combinatorial peptide ligand libraries fractionation of proteins in L. japonica immature flower buds.

Supplemental Figure 2. Schematic workflow for polyethylene glycol fractionation of proteins in $L$. japonica immature flower buds.

Supplemental Figure 3. Proteins identified in L. japonica immature flower buds using the combinatorial peptide ligand libraries fractionation (CPLL) method with different elution pHs.

Supplemental Figure 4. Major and minor proteins identified in L. japonica immature flower buds using the combinatorial peptide ligand libraries fractionation (CPLL) method.

Supplemental Figure 5. Proteins identified in L. japonica immature flower buds using polyethylene glycol fractionation.

Supplemental Table 1. Proteins Identified in L. japonica Immature Flower Buds at Fraction of Elution of pH 4 Fraction using Combinatorial Peptide Ligand Libraries Fractionation. P. S8

Supplemental Table 2. Proteins Identified in L. japonica Immature Flower Buds in the Flowthrough pH 4 Fraction using Combinatorial Peptide Ligand Libraries Fractionation. P. S16

Supplemental Table 3. Proteins of L. japonica Immature Flower Buds Identified in the $\mathrm{pH} 7$

Elution Fraction from Combinatorial Peptide Ligand Libraries Fractionation.

P. S25

Supplemental Table 4. Proteins of L. japonica Immature Flower Buds Identified in the Flow- 
through Fraction at pH 7 using Combinatorial Peptide Ligand Libraries Fractionation.

Supplemental Table 5. Proteins of L. japonica Immature Flower Buds Identified in the pH 9 Elution Fraction using Combinatorial Peptide Ligand Libraries Fractionation.

Supplemental Table 6. Proteins of L. japonica Immature Flower Buds Identified in in the Flowthrough pH 9 Fraction using Combinatorial Peptide Ligand Libraries Fractionation. P. S51

Supplemental Table 7. Proteins of L. japonica Immature Flower Buds Identified in Fraction 1 using Polyethylene Glycol Fractionation.

Supplemental Table 8. Proteins of L. japonica Immature Flower Buds Identified in Fraction 2 using Polyethylene Glycol Fractionation.

Supplemental Table 9. Proteins of L. japonica Immature Flower Buds Identified in Fraction 3 using Polyethylene Glycol Fractionation.

Supplemental Table 10. Crude Extract Proteins Identified in L. japonica Immature Flower Buds.

Supplemental Table 11. Proteins Identified in L. japonica Immature Flower Buds using Combinatorial Peptide Ligand Libraries Fractionation.

Supplemental Table 12. Proteins Identified in L. japonica Immature Flower Buds using Polyethylene Glycol Fractionation.

Supplemental Table 13. Proteins Identified in Cerasus Flower Buds. 
Supplemental Figure 1

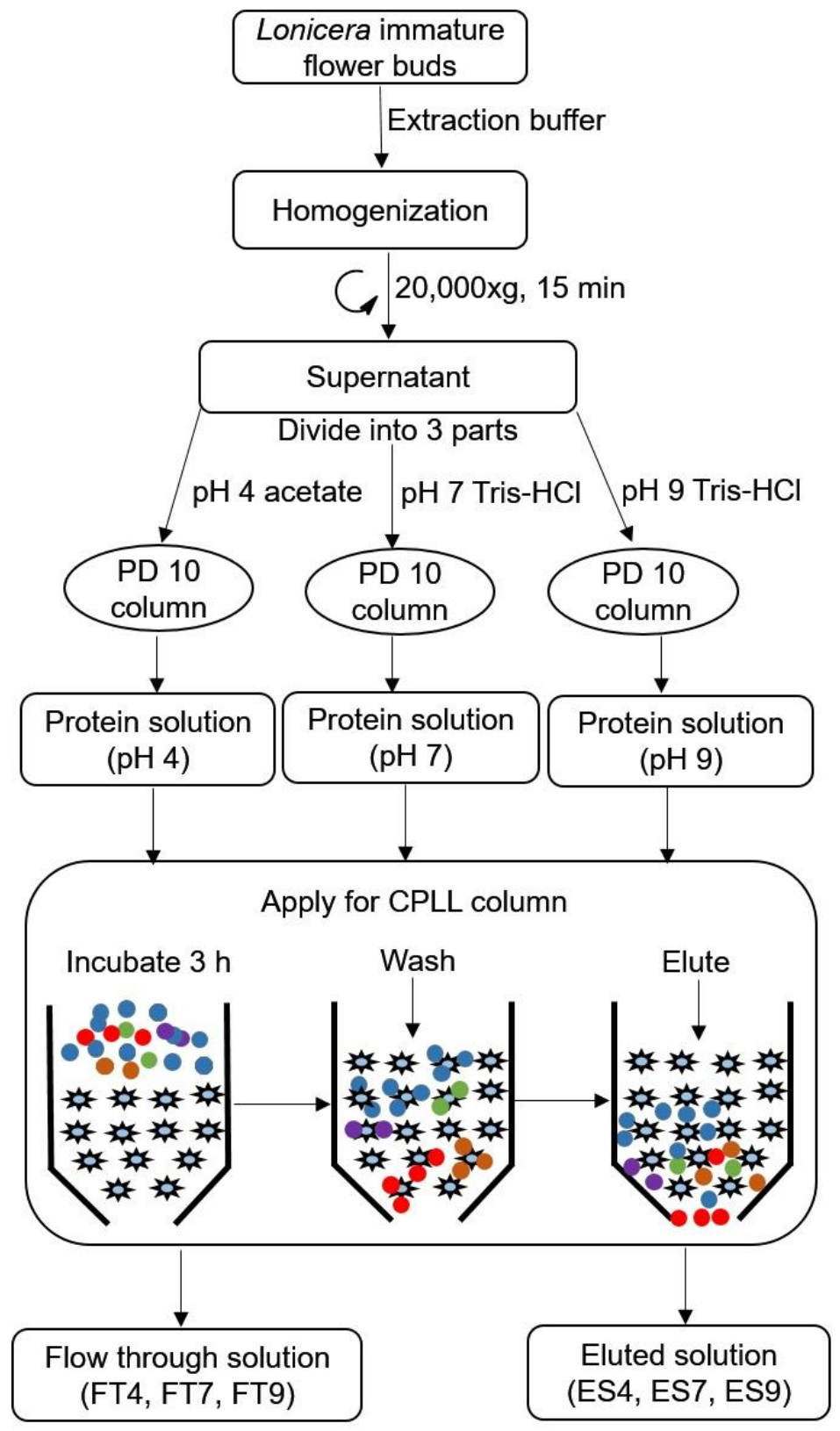

Supplemental Figure 1. Schematic workflow of the combinatorial peptide ligand libraries (CPLL) fractionation of proteins in $L$. japonicra immature flower buds. L. japonicra immature flower buds were ground to a powder in liquid nitrogen and homogenized in extraction buffer. After centrifugation, the supernatant was separated into 3 equal parts (for fractionation at $\mathrm{pH} 4,7$, and 9) for buffer exchange in PD 10 columns and was incubated in the CPLL columns for $3 \mathrm{~h}$. After centrifugation, the solution from CPLL columns was collected as flow-through solution (FT) and SDS solution was added to the columns to obtain eluted solution (ES). 
Supplemental Figure 2

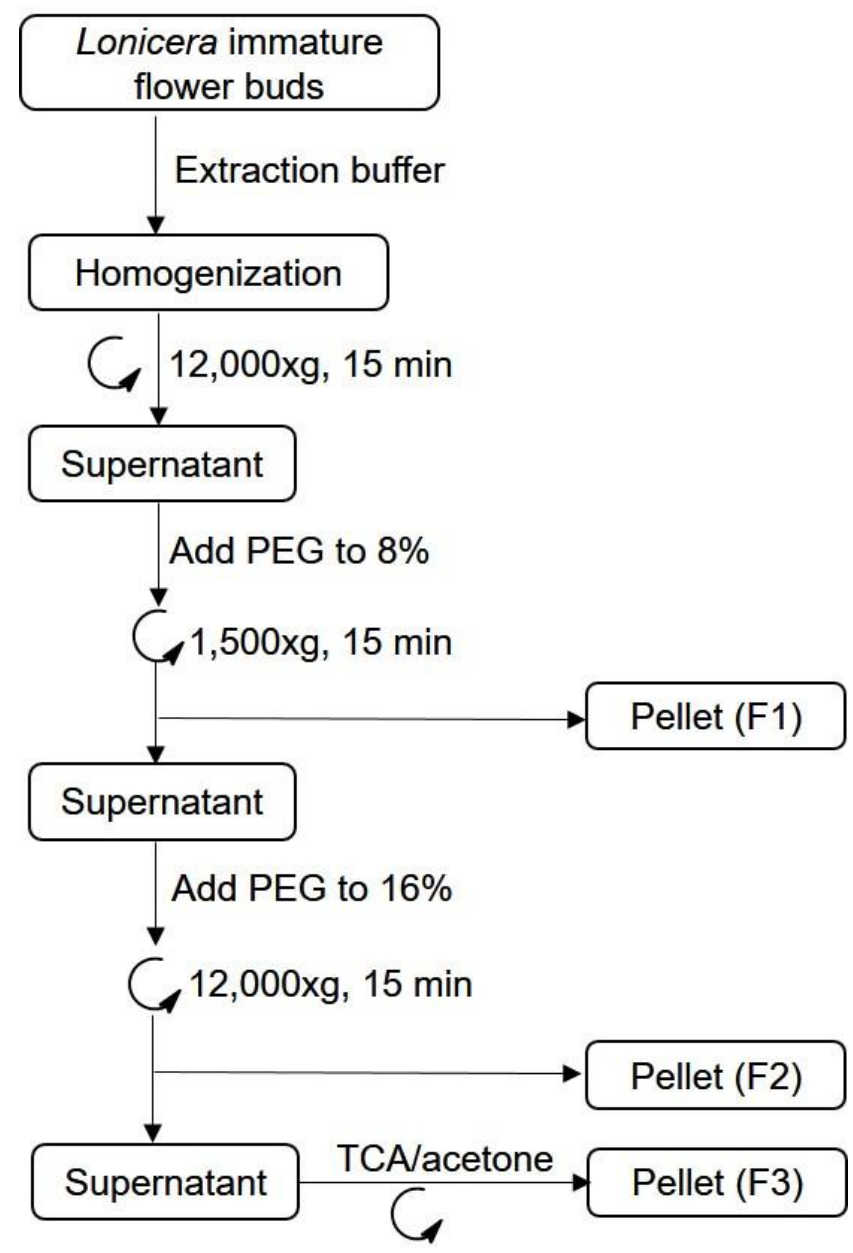

Supplemental Figure 2. Schematic workflow of the polyethylene glycol fractionation of proteins in L. japonicra immature flower buds. L. japonicra immature flower buds were ground to a powder in liquid nitrogen and homogenized in extraction buffer. After homogenization, the supernatant was mixed with $8 \%$ PEG and 16\% PEG, and 8\% PEG pellet (F1), 16\% PEG pellet (F2), and supernatant fractions (F3) were then collected 


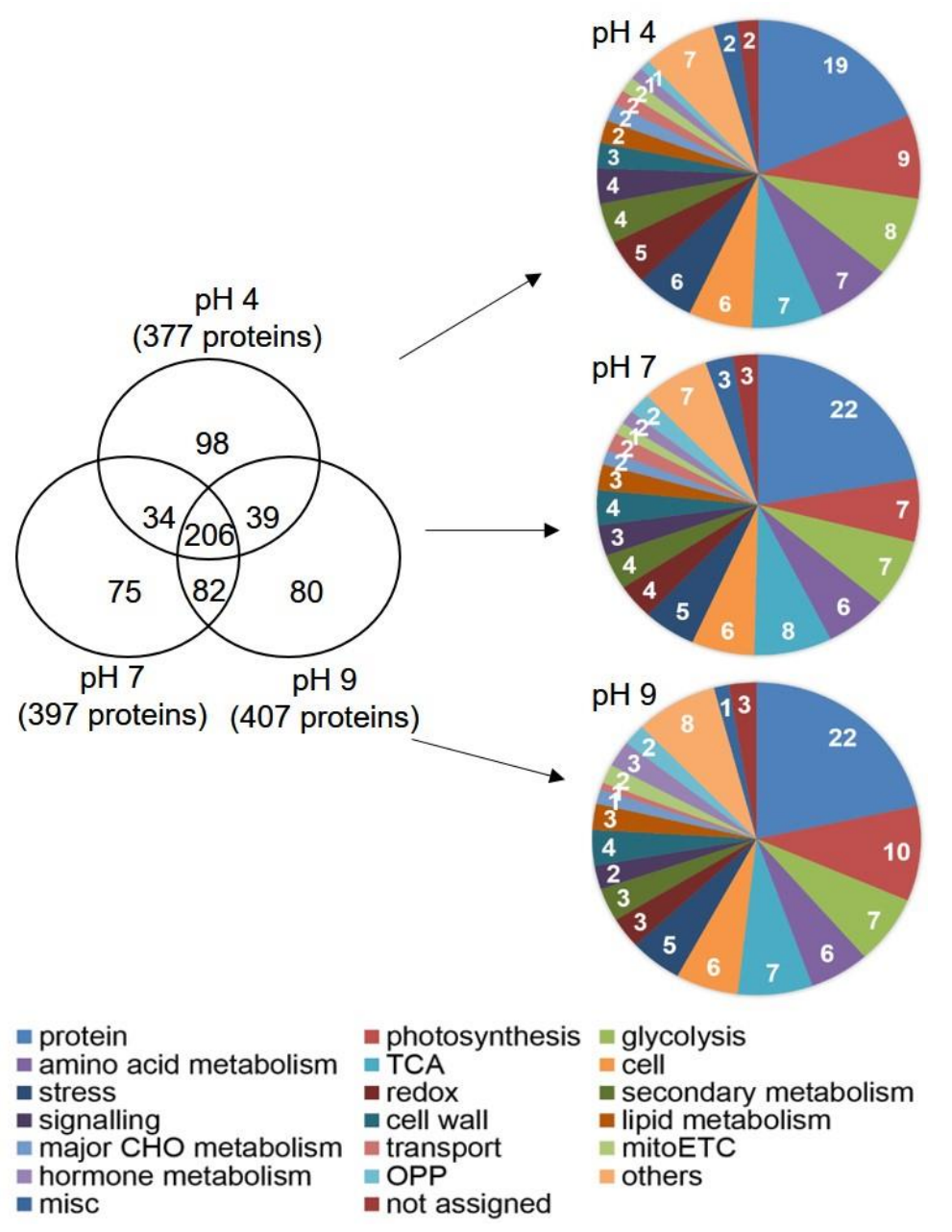

Supplemental Figure 3. Proteins identified in L. japonicra immature flower buds using the combinatorial peptide ligand libraries fractionation (CPLL) method with different elution pHs. Venn diagram showing the number of proteins that were identified among the three $\mathrm{pH}$ fractions. The identified proteins were categorized using MapMan bin codes. Pie graphs showing the percentage of proteins in each functional category. Abbreviations: protein, protein synthesis/posttranslational modification/folding/degradation; TCA, tricarboxylic acid cycle; cell, cell organization/vesicle transport/cycle/division; redox, redox ascorbate/glutathione metabolism; $\mathrm{CHO}$, carbohydrates; mitoETC, mitochondrial electron transport chains; OPP, oxidative pentose phosphate; others, containing C1-metabolism, co-factor/vitamin metabolism, development, gluconeogenesis, metal handling binding, minor $\mathrm{CHO}$ metabolism, N-metabolism, RNA and tetrapyrrole synthesis; and misc, miscellaneous 
Supplemental Figure 4

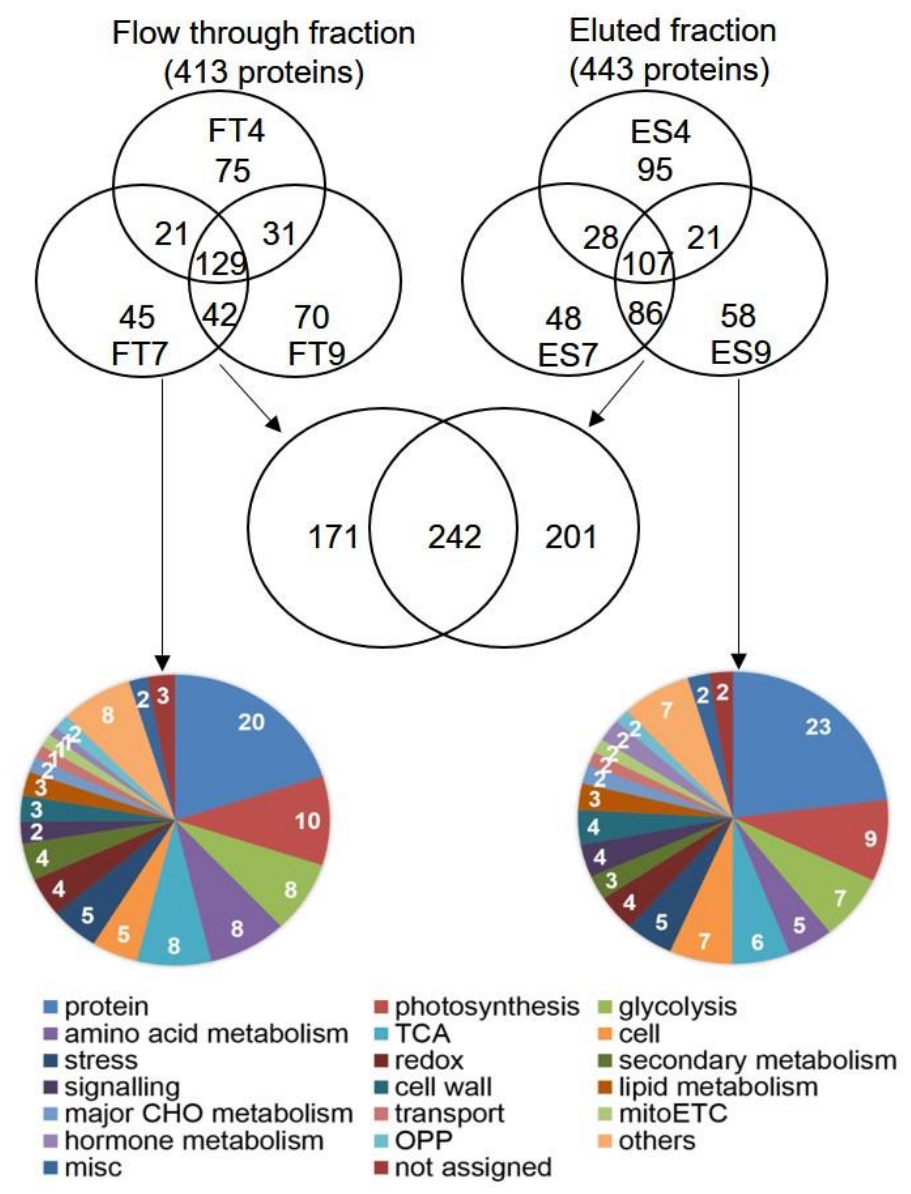

Supplemental Figure 4. Major and minor proteins identified in L. japonicra immature flower buds using the combinatorial peptide ligand libraries fractionation (CPLL) method. Venn diagram showing the number of proteins that were commonly identified in different flow-through fractions as major proteins and in eluted fractions as minor proteins. The identified major and minor proteins were categorized using MapMan bin codes. Pie graphs showing the percentage of proteins in each functional category. Abbreviations: protein, protein synthesis/posttranslational modification/folding/degradation; TCA, tricarboxylic acid cycle; cell, cell, cell organization/vesicle transport/cycle/division; redox, redox ascorbate/glutathione metabolism; $\mathrm{CHO}$, carbohydrates; mitoETC, mitochondrial electron transport chains; OPP, oxidative pentose phosphate; others, containing C1-metabolism, co-factor/vitamin metabolism, development, gluconeogenesis, metal handling binding, minor $\mathrm{CHO}$ metabolism, N-metabolism, RNA, and tetrapyrrole synthesis; and misc, miscellaneous. 
Supplemental Figure 5

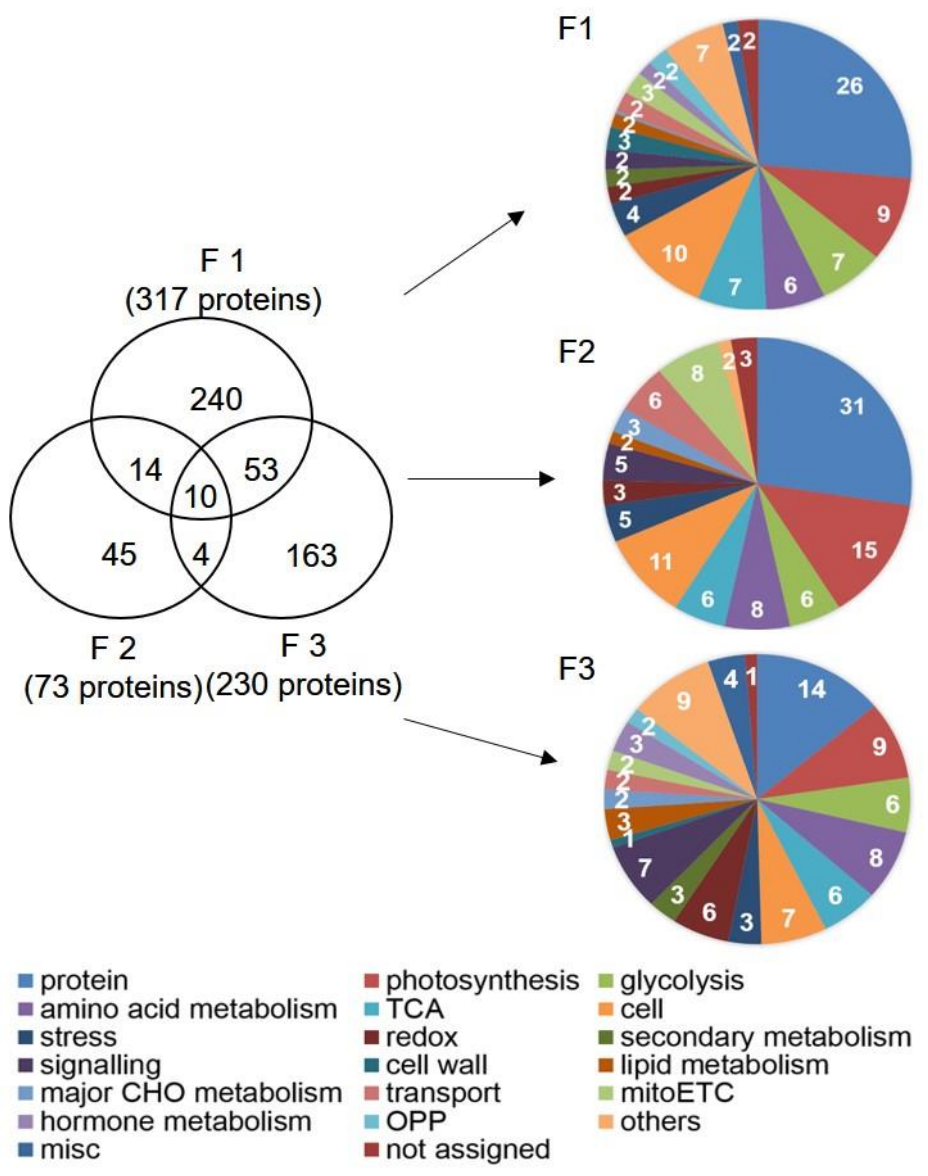

Supplemental Figure 5. Proteins identified in L. japonica flower buds using polyethylene glycol fractionation. Proteins extracted from L. japonica flower buds were fractioned using $8 \%$ and $16 \%$ PEG, and 8\% PEG pellet (F1), 16\% PEG pellet (F2), and supernatant fractions (F3) were collected. Proteins were reduced, alkylated, digested, and then analyzed using nanoLC-MS/MS. Venn diagram showing the number of proteins that were identified among the three fractions. The identified proteins were categorized using MapMan bin codes. Pie graphs showed the percentage of proteins in each functional category. Abbreviations: protein, protein synthesis/posttranslational modification/folding/degradation; TCA, tricarboxylic acid cycle; cell, cell organization/vesicle transport/cycle/division; redox, redox ascorbate/glutathione metabolism; CHO, carbohydrates; mitoETC, mitochondrial electron transport chains; OPP, oxidative pentose phosphate; others, containing C1-metabolism, co-factor/vitamin metabolism, development, gluconeogenesis, metal handling binding, minor $\mathrm{CHO}$ metabolism, $\mathrm{N}$-metabolism, RNA and tetrapyrrole synthesis; and misc, miscellaneous. 
Supplemental Table 1. Proteins Identified in L. japonica Immature Flower Buds at Fraction of Elution of pH 4 using Combinatorial Peptide Ligand Libraries Fractionation.

\begin{tabular}{|c|c|c|c|c|c|c|c|c|c|}
\hline No. & Protein ID ${ }^{\mathrm{a}}$ & Description & Species & M.P. ${ }^{\mathrm{b}}$ & Score & $\operatorname{Mol}(\%)^{\mathrm{c}}$ & $\mathrm{pI}$ & Mass (Da) & Function $^{\mathrm{d}}$ \\
\hline 1 & O81976 & 14-3-3 Protein & G. $\max$ & 30 & 395 & 3.73 & 4.21 & 7619 & signalling \\
\hline 2 & Q9ZQT0 & Actin & G. echinata & 22 & 282 & 1.88 & 6.19 & 17082 & cell \\
\hline 3 & E0D6S0 & Actin & G. bicolor & 63 & 1195 & 1.80 & 5.31 & 41913 & cell \\
\hline 4 & H1ZY49 & Translation elongation factor 1-alpha & L. maackii & 24 & 176 & 1.74 & 5.00 & 11619 & protein \\
\hline 5 & C4B8E5 & Glyceraldehyde-3-phosphate dehydrogenase & T. gesneriana & 122 & 3731 & 1.40 & 7.96 & 20964 & glycolysis \\
\hline 6 & B6TJ90 & Histone $\mathrm{H} 4$ & Z. mays & 19 & 201 & 1.38 & 9.67 & 20120 & cell \\
\hline 7 & Q6PKU2 & Putative glyceraldehyde-3-phosphate dehydrogenase & O. minor & 80 & 2047 & 1.18 & 7.09 & 22122 & glycolysis \\
\hline 8 & Q5GLI7 & Ribulose 1,5-bisphosphate carboxylase/oxygenase large subunit & E. ghellinckii & 80 & 785 & 1.10 & 6.89 & 48283 & photosynthesis \\
\hline 9 & Q75ZE0 & 14-3-3 e-1 protein & N. tabacum & 44 & 490 & 0.91 & 4.78 & 29479 & signalling \\
\hline 10 & D6C638 & Ribulose-1,5-bisphosphate carboxylase/oxygenase large subunit & Psychotria & 55 & 629 & 0.88 & 6.58 & 50770 & photosynthesis \\
\hline 11 & F8WL60 & Beta actin & R. stylosa & 35 & 774 & 0.87 & 5.27 & 33424 & cell \\
\hline 12 & Q9FX54 & At1g13440/F13B4_8 & A. thaliana & 132 & 3915 & 0.80 & 7.18 & 37004 & glycolysis \\
\hline 13 & J3RTS9 & Glyceraldehyde-3-phosphate dehydrogenase & C. pentagona & 148 & 3716 & 0.80 & 6.72 & 32382 & glycolysis \\
\hline 14 & F2D 884 & Predicted protein & H. vulgare & 108 & 1974 & 0.78 & 5.19 & 71476 & stress \\
\hline 15 & Q9M6B3 & Malate dehydrogenase & $V$. vinifera & 54 & 1609 & 0.74 & 8.62 & 37137 & TCA \\
\hline 16 & F2CYA6 & Predicted protein & H. vulgare & 93 & 1319 & 0.57 & 6.06 & 17119 & misc \\
\hline 17 & Q9SW73 & Isocitrate dehydrogenase [NADP] & C. limon & 21 & 400 & 0.56 & 6.95 & 46823 & TCA \\
\hline 18 & Q9FEM4 & Catalase & B. pendula & 12 & 113 & 0.56 & 5.48 & 17247 & redox \\
\hline 19 & Q9TKG9 & Ribulose 1,5-bisphosphate carboxylase large subunit & R. tomentosum & 72 & 622 & 0.55 & 7.11 & 52772 & photosynthesis \\
\hline 20 & F2CRF1 & Predicted protein & H. vulgare & 46 & 387 & 0.54 & 4.88 & 29361 & signalling \\
\hline 21 & B3TLP8 & Ubiquitin extension protein-like protein & E. guineensis & 92 & 1286 & 0.53 & 9.79 & 17902 & protein \\
\hline 22 & B4FNW1 & Triosephosphate isomerase & Z. mays & 28 & 266 & 0.50 & 5.68 & 27236 & glycolysis \\
\hline 23 & Q08II7 & Heat shock protein 70 -like protein & L. sativa & 4 & 116 & 0.49 & 10.33 & 12539 & stress \\
\hline 24 & Q6B443 & $26 \mathrm{~S}$ proteasome beta subunit & L. minor & 4 & 87 & 0.49 & 9.72 & 12530 & protein \\
\hline 25 & Q4H1G1 & Adenosylhomocysteinase & B. vulgaris & 80 & 1044 & 0.48 & 6.19 & 54052 & amino acid metabolism \\
\hline 26 & D7NHW9 & 2-phospho-D-glycerate hydrolase & P. trifoliata & 54 & 1300 & 0.48 & 5.78 & 47986 & glycolysis \\
\hline 27 & K4ACE3 & Uncharacterized protein & S. italica & 59 & 999 & 0.46 & 6.09 & 35803 & TCA \\
\hline 28 & Q6T379 & Triosephosphate isomerase & S. chacoense & 21 & 390 & 0.44 & 5.99 & 27251 & glycolysis \\
\hline 29 & B3TLQ5 & $60 S$ ribosomal protein $\mathrm{L} 11$ & E. guineensis & 10 & 156 & 0.44 & 9.92 & 20950 & protein \\
\hline 30 & D0ELH5 & Peptidyl-prolyl cis-trans isomerase & C. sinensis & 10 & 178 & 0.44 & 8.46 & 18310 & cell \\
\hline 31 & A9PD17 & Predicted protein & P. trichocarpa & 30 & 771 & 0.43 & 5.82 & 47103 & protein \\
\hline
\end{tabular}




\begin{tabular}{|c|c|c|c|c|c|c|c|c|c|}
\hline 32 & D9ZBV9 & Malate dehydrogenase & S. globosa & 8 & 122 & 0.43 & 4.92 & 13766 & TCA \\
\hline 33 & K3XJN7 & Uncharacterized protein & S. italica & 25 & 309 & 0.43 & 7.85 & 35629 & TCA \\
\hline 34 & K4A6U5 & Uncharacterized protein & S. italica & 55 & 920 & 0.42 & 5.34 & 71710 & stress \\
\hline 35 & A9P8Q7 & Predicted protein & P. trichocarpa & 36 & 406 & 0.41 & 4.84 & 28824 & signalling \\
\hline 36 & D0EJY9 & Molecular chaperone Hsp90-3 & N. benthamiana & 60 & 901 & 0.40 & 5.02 & 80638 & stress \\
\hline 37 & B6TCZ3 & Malate dehydrogenase & Z. mays & 19 & 427 & 0.40 & 7.74 & 41394 & TCA \\
\hline 38 & $\mathrm{~F} 2 \mathrm{E} 4 \mathrm{C} 2$ & Predicted protein & H. vulgare & 59 & 895 & 0.40 & 5.25 & 72202 & stress \\
\hline 39 & B3TLL4 & Triosephosphate isomerase & E. guineensis & 26 & 214 & 0.39 & 6.23 & 27476 & glycolysis \\
\hline 40 & Q8L4S4 & Phosphoglycerate kinase & A. speltoides & 19 & 123 & 0.36 & 5.01 & 31433 & photosynthesis \\
\hline 41 & B2VQE0 & Methionine synthase & O. ramosa & 148 & 2387 & 0.36 & 6.40 & 84862 & amino acid metabolism \\
\hline 42 & B6EBD6 & Heat shock protein $90-2$ & G. $\max$ & 59 & 739 & 0.35 & 5.07 & 80392 & stress \\
\hline 43 & Q40463 & NTGB2 & N. tabacum & 5 & 178 & 0.35 & 6.19 & 15827 & signalling \\
\hline 44 & D2CJB9 & ATP synthase subunit beta & B. multifida & 21 & 395 & 0.35 & 5.05 & 51275 & photosynthesis \\
\hline 45 & O81830 & Putative uncharacterized protein AT4g27270 & A. thaliana & 10 & 219 & 0.34 & 6.79 & 22355 & lipid metabolism \\
\hline 46 & K3ZUR5 & Uncharacterized protein & S. italica & 11 & 325 & 0.33 & 6.24 & 36749 & co-factor and vitamine metabolism \\
\hline 47 & F2DAC4 & Predicted protein & H. vulgare & 3 & 82 & 0.31 & 7.18 & 12136 & not assigned \\
\hline 48 & B6SJZ8 & Glyceraldehyde-3-phosphate dehydrogenase, cytosolic & Z. mays & 83 & 2198 & 0.31 & 6.96 & 36614 & glycolysis \\
\hline 49 & $\mathrm{O} 22331$ & Glutamine synthetase & H. brasiliensis & 15 & 309 & 0.30 & 6.20 & 39504 & $\mathrm{~N}$-metabolism \\
\hline 50 & Q19TV8 & UDP-glucose pyrophosphorylase & C. melo & 30 & 402 & 0.30 & 7.17 & 52201 & glycolysis \\
\hline 51 & B6SHD3 & Malate dehydrogenase & Z. mays & 16 & 312 & 0.30 & 8.32 & 42623 & TCA \\
\hline 52 & D6PAY2 & Peptidyl-prolyl cis-trans isomerase & V. hybrid cultivar & 13 & 208 & 0.29 & 8.46 & 18582 & cell \\
\hline 53 & F2CQQ3 & Predicted protein & H. vulgare & 17 & 330 & 0.28 & 5.16 & 54310 & transport \\
\hline 54 & F2CR08 & Predicted protein & H. vulgare & 39 & 605 & 0.28 & 5.55 & 48427 & glycolysis \\
\hline 55 & A2V880 & $\mathrm{G}$ protein beta-subunit-like protein & N. tabacum & 5 & 74 & 0.28 & 7.09 & 16100 & signalling \\
\hline 56 & A8JJQ6 & Histone H2B & C. reinhardtii & 8 & 70 & 0.28 & 9.85 & 16544 & DNA \\
\hline 57 & O81609 & Nodule-enhanced malate dehydrogenase & P. sativum & 14 & 451 & 0.28 & 7.75 & 42106 & TCA \\
\hline 58 & Q5IA96 & Cytochrome oxidase subunit II & A. trichopoda & 2 & 98 & 0.27 & 4.75 & 9794 & mitoETC \\
\hline 59 & K3Z913 & Uncharacterized protein & S. italica & 15 & 289 & 0.27 & 9.47 & 26793 & cell \\
\hline 60 & A3QQY2 & Sucrose synthase & C. intybus & 50 & 387 & 0.26 & 6.39 & 92338 & major $\mathrm{CHO}$ metabolism \\
\hline 61 & G5DVX2 & Phosphoglycerate kinase & S. latifolia & 32 & 593 & 0.26 & 6.58 & 51352 & photosynthesis \\
\hline 62 & B3H4P2 & Glyceraldehyde-3-phosphate dehydrogenase (NADP+) (Phosphorylating) & A. thaliana & 13 & 192 & 0.26 & 7.77 & 37929 & photosynthesis \\
\hline 63 & H9NIE1 & Cell division cycle protein 48 & C. sinensis & 38 & 1116 & 0.26 & 5.29 & 90644 & cell \\
\hline
\end{tabular}




\begin{tabular}{|c|c|c|c|c|c|c|c|c|c|}
\hline 64 & F2D8B8 & Proteasome subunit alpha type & H. vulgare & 9 & 124 & 0.26 & 7.43 & 27434 & protein \\
\hline 65 & K3XL57 & Uncharacterized protein & S. italica & 22 & 202 & 0.26 & 5.48 & 27435 & glycolysis \\
\hline 66 & J9QAL9 & Ribosomal protein $\mathrm{L} 2$ & A. corniculatum & 7 & 94 & 0.25 & 10.23 & 21703 & protein \\
\hline 67 & E4MXI2 & mRNA, clone: RTFL01-39-D20 & T. halophila & 43 & 685 & 0.25 & 5.21 & 73890 & stress \\
\hline 68 & Q1KUX5 & 6-phosphogluconate dehydrogenase, decarboxylating & C. spinosa & 28 & 247 & 0.24 & 6.06 & 53777 & OPP \\
\hline 69 & A2Q5W0 & Alpha-tubulin & M. truncatula & 22 & 694 & 0.24 & 5.10 & 50457 & cell \\
\hline 70 & $\mathrm{O} 04428$ & Putative uncharacterized protein & C. paradisi & 34 & 396 & 0.24 & 5.59 & 32737 & biodegradation of xenobiotics. \\
\hline 71 & E4MWV7 & mRNA, clone: RTFL01-09-M07 & T. halophila & 2 & 106 & 0.24 & 7.42 & 10792 & protein \\
\hline 72 & Q069K2 & 5-methyltetrahydropteroyltriglutamate--homocysteine methyltransferase & N. suaveolens & 71 & 1087 & 0.24 & 6.62 & 85060 & amino acid metabolism \\
\hline 73 & B6T9G3 & Alpha-1,4-glucan-protein synthase 1 & Z. mays & 20 & 455 & 0.23 & 7.05 & 41278 & cell wall \\
\hline 74 & K3Z7G1 & Uncharacterized protein & S. italica & 11 & 130 & 0.22 & 6.60 & 39285 & biodegradation of xenobiotics. \\
\hline 75 & K3YQW5 & Uncharacterized protein & S. italica & 15 & 371 & 0.22 & 5.39 & 68637 & transport \\
\hline 76 & $\mathrm{O} 23254$ & Serine hydroxymethyltransferase & A. thaliana & 22 & 641 & 0.21 & 7.23 & 52141 & C1-metabolism \\
\hline 77 & Q9SRV5 & 5-methyltetrahydropteroyltriglutamate--homocysteine methyltransferase & A. thaliana & 94 & 1377 & 0.21 & 6.51 & 84873 & amino acid metabolism \\
\hline 78 & F2DTB2 & Triosephosphate isomerase & H. vulgare & 18 & 139 & 0.21 & 7.42 & 32679 & photosynthesis \\
\hline 79 & Q5ZF83 & Putative uncharacterized protein & P. major & 12 & 79 & 0.21 & 7.28 & 25390 & cell wall \\
\hline 80 & D7SY81 & Putative uncharacterized protein & $V$. vinifera & 4 & 88 & 0.21 & 5.48 & 12099 & stress \\
\hline 81 & Q9AXR6 & ATP:citrate lyase & C. аппиит & 23 & 360 & 0.20 & 7.39 & 66376 & TCA \\
\hline 82 & G9MA91 & Fructose-bisphosphate aldolase & L. grandiflorum & 8 & 260 & 0.20 & 6.28 & 37690 & glycolysis \\
\hline 83 & G5DXP1 & Fructose-bisphosphate aldolase & S. latifolia & 7 & 242 & 0.20 & 6.16 & 42771 & photosynthesis \\
\hline 84 & Q9SXX4 & Fructose-bisphosphate aldolase & N. paniculata & 9 & 300 & 0.20 & 7.33 & 42832 & photosynthesis \\
\hline 85 & B3TM36 & 40S ribosomal protein $\mathrm{S} 8$ & E. guineensis & 8 & 227 & 0.20 & 10.43 & 25347 & protein \\
\hline 86 & Q9ZV36 & Putative nucleotide-sugar dehydratase & A. thaliana & 15 & 245 & 0.20 & 8.46 & 38768 & cell wall \\
\hline 87 & A1BLP6 & Thioredoxin & M. truncatula & 9 & 50 & 0.19 & 6.16 & 12904 & redox \\
\hline 88 & Q9ATF4 & Ribosomal protein L33 & C. sativa & 6 & 199 & 0.19 & 10.58 & 12831 & protein \\
\hline 89 & K3XFR6 & Uncharacterized protein & S. italica & 31 & 421 & 0.19 & 7.46 & 66448 & TCA \\
\hline 90 & Q8MCY2 & Ribulose bisphosphate carboxylase large chain & V. baccifera & 22 & 223 & 0.19 & 6.64 & 48739 & photosynthesis \\
\hline 91 & E4MVY3 & mRNA, clone: RTFL01-01-M18 & T. halophila & 43 & 766 & 0.18 & 6.25 & 94734 & protein \\
\hline 92 & K3YR06 & Uncharacterized protein & S. italica & 20 & 258 & 0.18 & 6.16 & 64225 & protein \\
\hline 93 & K4A9V6 & Uncharacterized protein & S. italica & 25 & 528 & 0.18 & 5.10 & 50519 & cell \\
\hline 94 & D3GQL1 & Aconitate hydratase 3 & C. clementina & 40 & 577 & 0.18 & 6.30 & 98669 & TCA \\
\hline 95 & F2CQP8 & Predicted protein & H. vulgare & 13 & 142 & 0.18 & 5.49 & 32811 & biodegradation of xenobiotics. \\
\hline
\end{tabular}




\begin{tabular}{|c|c|c|c|c|c|c|c|c|c|}
\hline 96 & A0EJL8 & GDP-D-mannose-3',5'-epimerase & M. glabra & 28 & 283 & 0.18 & 6.21 & 42927 & redox \\
\hline 97 & B4G0K4 & Phosphoglycerate kinase & Z. mays & 15 & 296 & 0.18 & 5.87 & 42470 & glycolysis \\
\hline 98 & K3XXC5 & Uncharacterized protein & S. italica & 26 & 286 & 0.18 & 7.06 & 42409 & glycolysis \\
\hline 99 & K4A7I4 & Uncharacterized protein & S. italica & 15 & 400 & 0.17 & 5.40 & 61666 & photosynthesis \\
\hline 100 & F2CTZ5 & Predicted protein & H. vulgare & 12 & 433 & 0.17 & 5.23 & 68752 & transport \\
\hline 101 & Q9FE12 & Peroxiredoxin & P. vulgaris & 13 & 238 & 0.17 & 5.33 & 28776 & redox \\
\hline 102 & Q8LG68 & UDP-glucose 6-dehydrogenase & A. thaliana & 15 & 293 & 0.17 & 6.18 & 53591 & stress \\
\hline 103 & Q8LC80 & Putative calcium-binding protein, calreticulin & A. thaliana & 19 & 472 & 0.17 & 4.53 & 48385 & signalling \\
\hline 104 & G7L7D9 & Profilin & M. truncatula & 8 & 40 & 0.17 & 5.40 & 14401 & cell \\
\hline 105 & Q4H1G2 & Methionine synthase & B. vulgaris & 77 & 1436 & 0.17 & 6.46 & 88546 & amino acid metabolism \\
\hline 106 & $\mathrm{~A} 2 \mathrm{PZC} 2$ & UDP-Glucose:protein transglucosylase & C. reinhardtii & 9 & 75 & 0.16 & 6.33 & 39846 & cell wall \\
\hline 107 & B4FPK8 & Cytochrome b5 & Z. mays & 4 & 92 & 0.16 & 5.30 & 14958 & redox \\
\hline 108 & K3ZW60 & Uncharacterized protein & S. italica & 14 & 172 & 0.16 & 9.76 & 29913 & protein \\
\hline 109 & H2BBB1 & ATP synthase subunit alpha, chloroplastic & P. diguetii & 11 & 189 & 0.16 & 5.74 & 55710 & photosynthesis \\
\hline 110 & G3JZV4 & Sucrose synthase & O. ramosa & 40 & 562 & 0.16 & 6.74 & 92420 & major $\mathrm{CHO}$ metabolism \\
\hline 111 & K3YR24 & Uncharacterized protein & S. italica & 11 & 226 & 0.16 & 7.91 & 62012 & protein \\
\hline 112 & Q8GZR6 & GcpE & S. lycopersicum & 22 & 205 & 0.16 & 6.11 & 82805 & secondary metabolism \\
\hline 113 & G0WP59 & Superoxide dismutase $[\mathrm{Cu}-\mathrm{Zn}]$ & W. somnifera & 8 & 192 & 0.15 & 9.04 & 15782 & redox \\
\hline 114 & E5GBS0 & 40 S ribosomal protein $\mathrm{S} 24$ & C. melo & 6 & 136 & 0.15 & 10.64 & 15761 & protein \\
\hline 115 & Q0W9E2 & Beta chaperonin 60 & S. commersonii & 18 & 396 & 0.15 & 5.64 & 63333 & protein \\
\hline 116 & B4FH62 & NAD-dependent epimerase/dehydratase & Z. mays & 10 & 220 & 0.15 & 7.27 & 31917 & not assigned \\
\hline 117 & A5JPK7 & Monodehydroascorbate reductase & V. vinifera & 24 & 157 & 0.15 & 6.24 & 47478 & redox \\
\hline 118 & A8MRE8 & Triosephosphate isomerase & A. thaliana & 13 & 316 & 0.15 & 7.49 & 32458 & photosynthesis \\
\hline 119 & B3TLL5 & 40S ribosomal protein S19 & E. guineensis & 10 & 57 & 0.15 & 10.04 & 16202 & protein \\
\hline 120 & K3YZ28 & Uncharacterized protein & S. italica & 4 & 149 & 0.15 & 10.59 & 15971 & protein \\
\hline 121 & F2DAU4 & Predicted protein & H. vulgare & 28 & 258 & 0.14 & 7.56 & 62696 & protein \\
\hline 122 & F2CWX1 & Predicted protein & H. vulgare & 25 & 299 & 0.14 & 6.10 & 86113 & protein \\
\hline 123 & B7UCR9 & 1-deoxy-d-xylulose 5-phosphate reductoisomerase & S. miltiorrhiza & 10 & 107 & 0.14 & 6.40 & 51960 & secondary metabolism \\
\hline 124 & Q8GZD5 & Xyloglucan endotransglycosylase & P. tremula & 16 & 246 & 0.14 & 7.75 & 34481 & cell wall \\
\hline 125 & K3ZW85 & Uncharacterized protein & S. italica & 15 & 74 & 0.14 & 10.35 & 29312 & protein \\
\hline 126 & B9RZK1 & Major allergen Pru ar, putative & R. communis & 2 & 32 & 0.14 & 4.98 & 17294 & stress \\
\hline 127 & Q7XAE2 & Putative fructokinase 2 & P. integrifolia & 23 & 214 & 0.14 & 5.35 & 35181 & major $\mathrm{CHO}$ metabolism \\
\hline
\end{tabular}




\begin{tabular}{|c|c|c|c|c|c|c|c|c|c|}
\hline 128 & B3TM23 & 40S ribosomal protein $\mathrm{S} 4$ & E. guineensis & 23 & 250 & 0.14 & 10.24 & 30023 & protein \\
\hline 129 & A1YUL9 & Importin subunit alpha & N. benthamiana & 8 & 166 & 0.13 & 5.38 & 59114 & protein \\
\hline 130 & Q7XJH9 & Transaldolase & S. lycopersicum & 13 & 101 & 0.13 & 5.96 & 48062 & OPP \\
\hline 131 & E4MXI1 & mRNA, clone: RTFL01-39-B07 & T. halophila & 11 & 249 & 0.13 & 6.98 & 48063 & photosynthesis \\
\hline 132 & $\mathrm{~F} 2 \mathrm{CW} 31$ & Predicted protein & H. vulgare & 12 & 229 & 0.13 & 6.21 & 63075 & glycolysis \\
\hline 133 & E3NYI6 & Peptidyl-prolyl cis-trans isomerase & A. diogoi & 10 & 59 & 0.13 & 8.44 & 18332 & cell \\
\hline 134 & B6TPE4 & Glutamate-1-semialdehyde 2,1-aminomutase & Z. mays & 7 & 88 & 0.13 & 6.55 & 50223 & tetrapyrrole synthesis \\
\hline 135 & K3YSB2 & Uncharacterized protein & S. italica & 10 & 80 & 0.13 & 8.63 & 50388 & amino acid metabolism \\
\hline 136 & F2EKH0 & Predicted protein & H. vulgare & 7 & 159 & 0.12 & 4.60 & 31771 & protein \\
\hline 137 & F2DLC2 & Proteasome subunit alpha type & H. vulgare & 7 & 173 & 0.12 & 4.68 & 26087 & protein \\
\hline 138 & E4MVQ7 & mRNA, clone: RTFL01-03-C18 & T. halophila & 12 & 211 & 0.12 & 6.60 & 46631 & redox \\
\hline 139 & Q1HG95 & Sucrose synthase & V. album & 33 & 162 & 0.12 & 6.09 & 92607 & major $\mathrm{CHO}$ metabolism \\
\hline 140 & E4MX73 & mRNA, clone: RTFL01-10-N01 & T. halophila & 5 & 135 & 0.12 & 5.83 & 39684 & TCA \\
\hline 141 & O65156 & Glutathione peroxidase & Z. aethiopica & 4 & 51 & 0.11 & 9.73 & 26916 & redox \\
\hline 142 & B6THG9 & $60 \mathrm{~S}$ ribosomal protein $\mathrm{L} 5-1$ & Z. mays & 10 & 183 & 0.11 & 9.32 & 34462 & protein \\
\hline 143 & E4MWP5 & mRNA, clone: RTFL01-15-G22 & T. halophila & 10 & 42 & 0.11 & 6.16 & 35258 & photosynthesis \\
\hline 144 & A8MRH4 & $60 \mathrm{~S}$ ribosomal protein $\mathrm{L} 7-2$ & A. thaliana & 5 & 40 & 0.11 & 10.10 & 21540 & protein \\
\hline 145 & K3Y5R7 & Uncharacterized protein & S. italica & 14 & 93 & 0.11 & 9.55 & 73000 & glycolysis \\
\hline 146 & Q9SXR9 & LeArcA1 protein & S. lycopersicum & 7 & 75 & 0.11 & 7.12 & 36363 & signalling \\
\hline 147 & А2PYH3 & Alpha chain of nascent polypeptide associated complex & N. benthamiana & 4 & 134 & 0.11 & 4.50 & 21911 & protein \\
\hline 148 & E4MWY6 & Glucose-6-phosphate isomerase & T. halophila & 7 & 185 & 0.11 & 5.60 & 67312 & glycolysis \\
\hline 149 & Q0MYQ7 & Germin-like protein 2 & $V$. vinifera & 2 & 117 & 0.10 & 9.58 & 22832 & stress \\
\hline 150 & K3XZE3 & Uncharacterized protein & S. italica & 2 & 118 & 0.10 & 5.27 & 23153 & protein \\
\hline 151 & Q2F951 & NADH dehydrogenase subunit 9 & O. sativa & 7 & 29 & 0.10 & 8.41 & 22694 & mitoETC \\
\hline 152 & K3XZA2 & Uncharacterized protein & S. italica & 8 & 82 & 0.10 & 7.82 & 23221 & cell \\
\hline 153 & Q34691 & ATP synthase subunit alpha & H. annuus & 12 & 139 & 0.10 & 6.60 & 55766 & mitoETC \\
\hline 154 & D7LW57 & Ketol-acid reductoisomerase & A. lyrata & 7 & 295 & 0.10 & 6.80 & 64346 & amino acid metabolism \\
\hline 155 & F2E797 & Predicted protein & H. vulgare & 6 & 90 & 0.10 & 4.98 & 47111 & TCA \\
\hline 156 & Q9SCB1 & Rab11 GTPase & S. lycopersicum & 8 & 81 & 0.10 & 5.72 & 24289 & signalling \\
\hline 157 & E5LCX1 & C14 cysteine protease & S. demissum & 3 & 157 & 0.10 & 11.49 & 23974 & protein \\
\hline 158 & K3ZWX8 & Uncharacterized protein & S. italica & 14 & 56 & 0.10 & 6.00 & 24135 & protein \\
\hline 159 & B3VDR8 & Plasma membrane proton pump & C. sativus & 22 & 120 & 0.10 & 6.96 & 105581 & transport \\
\hline
\end{tabular}




\begin{tabular}{|c|c|c|c|c|c|c|c|c|c|}
\hline 160 & E5F725 & Putative uncharacterized protein & E. parvulum & 3 & 180 & 0.10 & 7.11 & 42041 & cell wall \\
\hline 161 & B3TLP3 & Translation elongation factor EF-1 beta chain & E. guineensis & 10 & 112 & 0.09 & 6.89 & 24523 & protein \\
\hline 162 & B3TLY3 & Ribosomal protein L15 & E. guineensis & 6 & 242 & 0.09 & 4.69 & 24371 & protein \\
\hline 163 & H6VUT7 & Translation elongation factor & A. mongolicus & 6 & 152 & 0.09 & 11.49 & 25300 & protein \\
\hline 164 & K3Y9Y1 & Uncharacterized protein & S. italica & 5 & 41 & 0.09 & 6.23 & 24396 & protein \\
\hline 165 & B6TGL7 & Enolase & Z. mays & 2 & 101 & 0.09 & 4.63 & 50512 & glycolysis \\
\hline 166 & A9PAK9 & Putative uncharacterized protein & P. trichocarpa & 5 & 45 & 0.09 & 10.07 & 50623 & amino acid metabolism \\
\hline 167 & K3XVH0 & Uncharacterized protein & S. italica & 18 & 160 & 0.09 & 9.76 & 80203 & photosynthesis \\
\hline 168 & O78327 & Transketolase 1 & C. аппиит & 25 & 146 & 0.09 & 8.63 & 80398 & photosynthesis \\
\hline 169 & F2DTH4 & Adenylate kinase & H. vulgare & 5 & 99 & 0.09 & 7.81 & 26477 & nucleotide metabolism \\
\hline 170 & F2DP49 & Predicted protein & H. vulgare & 3 & 75 & 0.09 & 5.63 & 25913 & hormone metabolism \\
\hline 171 & B6ZK00 & Peroxisomal biogenesis factor 11 family protein & G. $\max$ & 2 & 110 & 0.09 & 6.62 & 26114 & cell \\
\hline 172 & G8HAB2 & PLP-dependent aminotransferase & P. somniferum & 7 & 130 & 0.09 & 6.62 & 53501 & amino acid metabolism \\
\hline 173 & F2DLJ7 & Predicted protein & H. vulgare & 2 & 110 & 0.09 & 7.77 & 44423 & hormone metabolism \\
\hline 174 & E4MY36 & mRNA, clone: RTFL01-37-B16 & T. halophila & 14 & 158 & 0.09 & 6.25 & 63555 & glycolysis \\
\hline 175 & Q9XEN8 & Annexin & N. tabacum & 7 & 124 & 0.09 & 5.53 & 36029 & cell \\
\hline 176 & С0Р9J6 & Aminoaldehyde dehydrogenase 1 & Z. mays & 4 & 83 & 0.08 & 5.76 & 55733 & secondary metabolism \\
\hline 177 & I6RZD4 & Putative NAD-dependent dehydrogenase 2 & E. coca & 2 & 71 & 0.08 & 5.45 & 27370 & protein \\
\hline 178 & B3TLW0 & Glutathione S-transferase & E. guineensis & 3 & 43 & 0.08 & 5.83 & 27569 & misc \\
\hline 179 & F2D3S8 & Serine hydroxymethyltransferase & H. vulgare & 17 & 63 & 0.08 & 8.21 & 56215 & C1-metabolism \\
\hline 180 & Q8H6B6 & Chaperone protein HtpG & X. viscosa & 21 & 335 & 0.08 & 5.02 & 92957 & stress \\
\hline 181 & C4NZX3 & Lipoxygenase & C. sinensis & 14 & 165 & 0.08 & 6.83 & 102365 & hormone metabolism \\
\hline 182 & Q8LDN8 & Atlg63180 & A. thaliana & 8 & 63 & 0.08 & 6.73 & 39171 & cell wall \\
\hline 183 & K3ZI40 & Uncharacterized protein & S. italica & 7 & 56 & 0.08 & 5.01 & 56750 & redox \\
\hline 184 & Q645M9 & Glyoxisomal malate dehydrogenase & S. lycopersicum & 5 & 125 & 0.08 & 7.94 & 38007 & gluconeogenesis \\
\hline 185 & B6U0V6 & Endoplasmin & Z. mays & 18 & 324 & 0.07 & 4.97 & 92831 & stress \\
\hline 186 & B7SA66 & Hsp90-like protein & D. glomerata & 15 & 266 & 0.07 & 4.97 & 92918 & stress \\
\hline 187 & E4MXR5 & mRNA, clone: RTFL01-40-M04 & T. halophila & 10 & 203 & 0.07 & 5.90 & 73489 & stress \\
\hline 188 & F2D269 & Predicted protein & H. vulgare & 20 & 240 & 0.07 & 5.04 & 73306 & stress \\
\hline 189 & Q6VWJ5 & Fructokinase 3 & S. lycopersicum & 5 & 90 & 0.07 & 5.80 & 41803 & major $\mathrm{CHO}$ metabolism \\
\hline 190 & F2DGF3 & Predicted protein & H. vulgare & 13 & 159 & 0.07 & 6.81 & 115601 & TCA \\
\hline 191 & Q40270 & RNA-binding protein & M. crystallinum & 2 & 47 & 0.07 & 7.49 & 32001 & RNA \\
\hline
\end{tabular}




\begin{tabular}{|c|c|c|c|c|c|c|c|c|c|}
\hline 192 & F6HGZ1 & Pectinesterase & V. vinifera & 6 & 74 & 0.07 & 4.79 & 63329 & cell wall \\
\hline 193 & Q6SKP4 & Ribosomal protein L3 & S. lycopersicum & 14 & 123 & 0.07 & 10.08 & 44836 & protein \\
\hline 194 & A0FKE6 & Chloroplast threonine deaminase 1 & S. lycopersicum & 5 & 58 & 0.07 & 6.37 & 66653 & amino acid metabolism \\
\hline 195 & K4AAQ4 & Uncharacterized protein & S. italica & 6 & 128 & 0.07 & 10.56 & 44399 & protein \\
\hline 196 & K4A6V8 & Uncharacterized protein & S. italica & 9 & 116 & 0.07 & 7.03 & 70549 & glycolysis \\
\hline 197 & F2CQX6 & Thioredoxin reductase & H. vulgare & 3 & 59 & 0.07 & 6.23 & 35031 & redox \\
\hline 198 & I0J3D8 & Proteasome subunit beta type & A. halleri & 2 & 103 & 0.07 & 5.87 & 33367 & protein \\
\hline 199 & F2E397 & Predicted protein & H. vulgare & 6 & 206 & 0.07 & 6.06 & 34933 & major $\mathrm{CHO}$ metabolism \\
\hline 200 & I6LE48 & Uncharacterized protein & D. excelsa & 4 & 48 & 0.07 & 5.03 & 33659 & development \\
\hline 201 & B6SUH6 & Non-cyanogenic beta-glucosidase & Z. mays & 21 & 47 & 0.07 & 5.80 & 56909 & misc \\
\hline 202 & F2E2M4 & Predicted protein & H. vulgare & 8 & 166 & 0.07 & 5.29 & 92002 & stress \\
\hline 203 & K3XJZ1 & Uncharacterized protein & S. italica & 4 & 33 & 0.06 & 9.39 & 35932 & protein \\
\hline 204 & Q93YG1 & Monodehydroascorbate reductase & M. crystallinum & 28 & 164 & 0.06 & 6.81 & 51944 & redox \\
\hline 205 & P93698 & Lipoxygenase & V. unguiculata & 13 & 179 & 0.06 & 6.49 & 102731 & hormone metabolism \\
\hline 206 & Q39986 & Cysteine proteinase & H. $s p$. & 3 & 55 & 0.06 & 5.50 & 40006 & protein \\
\hline 207 & H9C956 & 3-deoxy-D-arabino-heptulosonate 7-phosphate synthase & N. benthamiana & 9 & 117 & 0.05 & 8.28 & 53191 & amino acid metabolism \\
\hline 208 & Q9ZNX1 & NAD-dependent isocitrate dehydrogenase & N. tabacum & 2 & 90 & 0.05 & 6.68 & 40651 & TCA \\
\hline 209 & A9ZMZ5 & Acetyl-CoA C-acetyltransferase & H. brasiliensis & 13 & 489 & 0.05 & 7.66 & 42039 & secondary metabolism \\
\hline 210 & B4FYM0 & Acetyl-CoA acetyltransferase, cytosolic 2 & Z. mays & 5 & 111 & 0.05 & 6.11 & 43450 & secondary metabolism \\
\hline 211 & F6H3T7 & Putative uncharacterized protein & V. vinifera & 10 & 104 & 0.05 & 7.52 & 40651 & not assigned \\
\hline 212 & B9HJ23 & Predicted protein & P. trichocarpa & 9 & 154 & 0.05 & 6.54 & 101700 & misc \\
\hline 213 & Q39834 & Clathrin heavy chain & G. $\max$ & 12 & 154 & 0.05 & 5.55 & 194430 & cell \\
\hline 214 & B6TVN4 & Phospholipase C & Z. mays & 3 & 51 & 0.05 & 7.99 & 46868 & signalling \\
\hline 215 & A9P9A2 & Putative uncharacterized protein & P. trichocarpa & 18 & 190 & 0.05 & 9.36 & 46272 & RNA \\
\hline 216 & E1Z980 & Putative uncharacterized protein & C. variabilis & 3 & 54 & 0.05 & 8.43 & 44716 & protein \\
\hline 217 & Q6L619 & Beta-galactosidase & R. sativus & 6 & 124 & 0.05 & 6.49 & 93664 & misc \\
\hline 218 & A8J6J6 & Acetyl-CoA acyltransferase & C. reinhardtii & 8 & 65 & 0.05 & 8.38 & 47670 & amino acid metabolism \\
\hline 219 & H9A1W2 & Acyl-activating enzyme 10 & C. sativa & 4 & 55 & 0.05 & 6.49 & 62013 & lipid metabolism \\
\hline 220 & G8HAB1 & PLP-dependent aminotransferase & P. somniferum & 3 & 49 & 0.04 & 7.28 & 50587 & secondary metabolism \\
\hline 221 & K4A9K9 & Uncharacterized protein & S. italica & 2 & 37 & 0.04 & 7.65 & 52168 & secondary metabolism \\
\hline 222 & C5WQZ5 & Putative uncharacterized protein Sb01g041210 & S. bicolor & 3 & 117 & 0.04 & 5.17 & 51802 & protein \\
\hline 223 & G3CHK8 & Eukaryotic translation initiation factor 3 subunit $\mathrm{E}$ & P. trifoliata & 3 & 41 & 0.04 & 6.14 & 51916 & protein \\
\hline
\end{tabular}




\begin{tabular}{|c|c|c|c|c|c|c|c|c|c|}
\hline 224 & $\mathrm{~K} 3 \mathrm{XX} 43$ & Uncharacterized protein & S. italica & 9 & 110 & 0.04 & 7.09 & 48119 & protein \\
\hline 225 & F2E6F3 & Coatomer subunit gamma & H. vulgare & 4 & 165 & 0.04 & 6.29 & 100353 & cell \\
\hline 226 & B9S7T6 & Transaminase mtnE, putative & R. communis & 7 & 71 & 0.04 & 5.83 & 50909 & amino acid metabolism \\
\hline 227 & Q43057 & Aspartate aminotransferase & P. miliaceum & 4 & 33 & 0.04 & 7.33 & 48154 & amino acid metabolism \\
\hline 228 & B5LAT6 & Dihydrolipoyl dehydrogenase & C. аппиит & 7 & 52 & 0.04 & 6.86 & 54133 & TCA \\
\hline 229 & K3YRV8 & Uncharacterized protein & S. italica & 8 & 61 & 0.04 & 7.56 & 55802 & TCA \\
\hline 230 & $\mathrm{O} 24135$ & Citrate synthase & N. tabacum & 5 & 123 & 0.04 & 8.76 & 52744 & TCA \\
\hline 231 & K4A938 & Uncharacterized protein & S. italica & 2 & 49 & 0.04 & 6.60 & 53547 & nucleotide metabolism \\
\hline 232 & F2EG33 & Predicted protein & H. vulgare & 9 & 92 & 0.04 & 7.27 & 52947 & lipid metabolism \\
\hline 233 & F2CS51 & Pyruvate kinase & H. vulgare & 7 & 23 & 0.04 & 6.79 & 56102 & glycolysis \\
\hline 234 & K3XWI6 & Uncharacterized protein & S. italica & 8 & 161 & 0.04 & 7.85 & 55489 & glycolysis \\
\hline 235 & Q9S768 & Alanine aminotransferase & Oryza sativa & 7 & 185 & 0.04 & 6.65 & 53130 & amino acid metabolism \\
\hline 236 & B2KNE6 & Phospholipase D & H. anпииs & 10 & 162 & 0.04 & 5.52 & 92315 & lipid metabolism \\
\hline 237 & K3Y4M8 & Uncharacterized protein & S. italica & 6 & 151 & 0.04 & 5.39 & 194893 & cell \\
\hline 238 & J3MFV3 & Lipoxygenase & O. brachyantha & 11 & 35 & 0.04 & 6.77 & 103742 & hormone metabolism \\
\hline 239 & K3YPS9 & Uncharacterized protein & S. italica & 12 & 166 & 0.04 & 5.24 & 97789 & protein \\
\hline 240 & B2Z6P0 & 4-Coumarate:CoA ligase & P. trichocarpa & 2 & 53 & 0.04 & 5.69 & 59541 & secondary metabolism \\
\hline 241 & F2DUH3 & Predicted protein & H. vulgare & 4 & 59 & 0.04 & 5.63 & 60964 & protein \\
\hline 242 & Q9SA89 & FAD-binding and BBE domain-containing protein & A. thaliana & 4 & 54 & 0.04 & 8.60 & 60060 & not assigned \\
\hline 243 & Q8L5C2 & $110 \mathrm{kDa} 4 \mathrm{SNc}$-Tudor domain protein & P. sativum & 5 & 63 & 0.03 & 7.36 & 108600 & RNA \\
\hline 244 & Q38766 & Victorin binding protein & A. sativa & 10 & 82 & 0.03 & 6.96 & 112298 & photosynthesis \\
\hline 245 & J9Z414 & Beta-D-glucan exohydolase & E. guineensis & 2 & 46 & 0.03 & 7.88 & 69462 & cell wall \\
\hline 246 & K4A6E4 & Uncharacterized protein & S. italica & 5 & 31 & 0.03 & 5.16 & 83215 & protein \\
\hline 247 & Q2LFC1 & AGO4-2 & N. benthamiana & 7 & 80 & 0.02 & 8.09 & 102331 & RNA \\
\hline 248 & Q9ZVY6 & $\mathrm{T} 25 \mathrm{~N} 20.17$ & A. thaliana & 5 & 48 & 0.02 & 6.52 & 90732 & protein \\
\hline 249 & A2JGX1 & Beta-galactosidase & S. lycopersicum & 4 & 51 & 0.02 & 8.78 & 98181 & misc \\
\hline 250 & Q5BLY1 & Plastid alpha-amylase & A. chinensis & 8 & 59 & 0.02 & 6.30 & 101618 & major $\mathrm{CHO}$ metabolism \\
\hline 251 & A8II37 & Predicted protein & C. reinhardtii & 11 & 86 & 0.02 & 5.58 & 101172 & fermentation \\
\hline
\end{tabular}

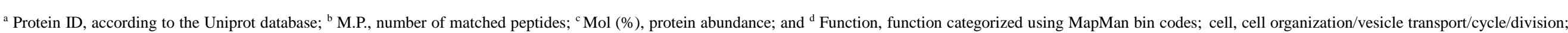

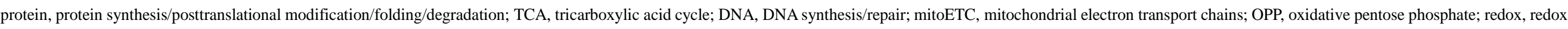
ascorbate/glutathione metabolism; RNA, RNA processing/regulation of transcription; and misc, miscellaneous. 
Supplemental Table 2. Proteins Identified in L. japonica Immature Flower Buds at Fraction of Flow Through of pH 4 using Combinatorial Peptide Ligand Libraries Fractionation.

\begin{tabular}{|c|c|c|c|c|c|c|c|c|c|}
\hline No. & Protein ID ${ }^{\mathrm{a}}$ & Description & Species & M.P. ${ }^{b}$ & Score & $\operatorname{Mol}(\%)^{\mathrm{c}}$ & $\mathrm{pI}$ & Mass (Da) & Function $^{\mathrm{d}}$ \\
\hline 1 & C4B8E5 & Glyceraldehyde-3-phosphate dehydrogenase & T. gesneriana & 144 & 4605 & 1.69 & 7.96 & 20964 & glycolysis \\
\hline 2 & H1ZY49 & Translation elongation factor 1-alpha & L. maackii & 20 & 96 & 1.48 & 5.00 & 11619 & protein \\
\hline 3 & Q6PKU2 & Putative glyceraldehyde-3-phosphate dehydrogenase & O. minor & 91 & 2316 & 1.28 & 7.09 & 22122 & glycolysis \\
\hline 4 & C6GFP3 & ATP synthase subunit B. & G. hirsutum & 148 & 2953 & 1.21 & 6.29 & 59987 & mitoETC \\
\hline 5 & Q9M6B3 & Malate dehydrogenase & V. vinifera & 133 & 3075 & 1.16 & 8.62 & 37137 & TCA \\
\hline 6 & Q41534 & ATP synthase subunit B. & T. aestivum & 132 & 2910 & 1.10 & 5.86 & 59326 & mitoETC \\
\hline 7 & H6T8A4 & ATP synthase subunit B. & M. poeppigii & 50 & 1017 & 0.96 & 6.33 & 52110 & photosynthesis \\
\hline 8 & Q08II7 & Heat shock protein 70 -like protein & L. sativa & 8 & 405 & 0.92 & 10.33 & 12539 & stress \\
\hline 9 & F2D884 & Predicted protein & H. vulgare & 98 & 1736 & 0.84 & 5.19 & 71476 & stress \\
\hline 10 & Q944T3 & Glyceraldehyde 3-phosphate dehydrogenase 2 & F. ananassa & 81 & 2694 & 0.78 & 9.20 & 13954 & glycolysis \\
\hline 11 & E5GBV9 & Glyceraldehyde-3-phosphate dehydrogenase & C. melo & 149 & 2823 & 0.73 & 6.62 & 36471 & glycolysis \\
\hline 12 & Q1A5W2 & Ribulose-1,5-bisphosphate carboxylase/oxygenase large subunit & B. hatcheri & 69 & 542 & 0.71 & 6.43 & 47148 & photosynthesis \\
\hline 13 & K4ACE3 & Uncharacterized protein & S. italica & 93 & 1641 & 0.70 & 6.09 & 35803 & TCA \\
\hline 14 & J3RTS9 & Glyceraldehyde-3-phosphate dehydrogenase & C. pentagona & 166 & 4476 & 0.69 & 6.73 & 32382 & glycolysis \\
\hline 15 & Q645N1 & Malate dehydrogenase & S. lycopersicum & 65 & 1409 & 0.68 & 8.72 & 36357 & TCA \\
\hline 16 & B4FNW1 & Triosephosphate isomerase & Z. mays & 28 & 344 & 0.63 & 5.68 & 27236 & glycolysis \\
\hline 17 & E5GBV8 & Glyceraldehyde-3-phosphate dehydrogenase & C. melo & 109 & 2853 & 0.59 & 7.12 & 36754 & glycolysis \\
\hline 18 & Q8L4S4 & Phosphoglycerate kinase & A. speltoides & 32 & 396 & 0.57 & 5.01 & 31433 & photosynthesis \\
\hline 19 & K3XJN7 & Uncharacterized protein & S. italica & 65 & 1004 & 0.56 & 7.85 & 35629 & TCA \\
\hline 20 & Q5ZFR7 & Malate dehydrogenase & P. major & 81 & 1429 & 0.55 & 6.54 & 36026 & TCA \\
\hline 21 & K3Z4G6 & Uncharacterized protein & S. italica & 83 & 1517 & 0.52 & 5.25 & 71367 & stress \\
\hline 22 & E4MW44 & mRNA, clone: RTFL01-05-I15 & T. halophila & 36 & 462 & 0.52 & 5.58 & 42078 & cell \\
\hline 23 & Q6T379 & Triosephosphate isomerase & S. chacoense & 23 & 470 & 0.52 & 5.99 & 27251 & glycolysis \\
\hline 24 & B3TLL4 & Triosephosphate isomerase & E. guineensis & 24 & 247 & 0.51 & 6.23 & 27476 & glycolysis \\
\hline 25 & K3YQW5 & Uncharacterized protein & S. italica & 34 & 805 & 0.51 & 5.39 & 68637 & transport \\
\hline 26 & Q9T2N3 & Chaperonin-60 alpha 1 fragment & B. napus & 2 & 45 & 0.49 & 4.01 & 6261 & protein \\
\hline 27 & Q40463 & NTGB2 & N. tabacum & 7 & 153 & 0.47 & 6.19 & 15827 & signalling \\
\hline 28 & A0ZQA9 & ATP synthase subunit B. & P. baccatum & 31 & 334 & 0.46 & 5.40 & 52897 & photosynthesis \\
\hline 29 & K3ZUR5 & Uncharacterized protein & S. italica & 22 & 758 & 0.46 & 6.24 & 36749 & co-factor and vitamine metabolism \\
\hline 30 & D9ZBV9 & Malate dehydrogenase & S. globosa & 13 & 265 & 0.45 & 4.92 & 13766 & TCA \\
\hline 31 & B6SHD3 & Malate dehydrogenase & Z. mays & 62 & 767 & 0.44 & 8.32 & 42623 & TCA \\
\hline
\end{tabular}




\begin{tabular}{|c|c|c|c|c|c|c|c|c|c|}
\hline 32 & B2VQE0 & Methionine synthase & O. ramosa & 171 & 2629 & 0.40 & 6.40 & 84862 & amino acid metabolism \\
\hline 33 & Q04130 & G.-rich protein & S. lycopersicum & 2 & 32 & 0.40 & 9.98 & 7327 & not assigned \\
\hline 34 & Q19TV8 & UDP-glucose pyrophosphorylase & C. melo & 29 & 455 & 0.39 & 7.17 & 52201 & glycolysis \\
\hline 35 & Q9SAT7 & Superoxide dismutase $[\mathrm{Cu}-\mathrm{Zn}]$ & P. tremuloides & 6 & 77 & 0.39 & 5.82 & 15420 & redox \\
\hline 36 & I3NVX2 & V-type ATPase subunit A & S. europaea & 31 & 548 & 0.38 & 5.20 & 68845 & transport \\
\hline 37 & G0WP59 & Superoxide dismutase $[\mathrm{Cu}-\mathrm{Zn}]$ & W. somnifera & 10 & 224 & 0.38 & 5.64 & 15782 & redox \\
\hline 38 & Q7DM38 & Ribulose bisphosphate carboxylase small chain & P. vulgaris & 28 & 116 & 0.37 & 8.43 & 15877 & photosynthesis \\
\hline 39 & K3XL57 & Uncharacterized protein & S. italica & 21 & 171 & 0.36 & 5.48 & 27435 & glycolysis \\
\hline 40 & Q8VWP3 & Glyceraldehyde-3-phosphate dehydrogenase & C. аппиит & 9 & 122 & 0.35 & 6.70 & 33465 & photosynthesis \\
\hline 41 & O81609 & Nodule-enhanced malate dehydrogenase & P. sativum & 25 & 804 & 0.35 & 7.75 & 42106 & TCA \\
\hline 42 & B6SJZ8 & Glyceraldehyde-3-phosphate dehydrogenase, cytosolic & Z. mays & 100 & 2845 & 0.35 & 6.96 & 36614 & glycolysis \\
\hline 43 & A8MQP1 & Adenosylhomocysteinase 1 & A. thaliana & 35 & 283 & 0.33 & 5.16 & 36022 & amino acid metabolism \\
\hline 44 & G5DVX2 & Phosphoglycerate kinase & S. latifolia & 54 & 964 & 0.32 & 6.58 & 51352 & photosynthesis \\
\hline 45 & Q8LC80 & Putative calcium-binding protein, calreticulin & A. thaliana & 35 & 742 & 0.32 & 4.53 & 48385 & signalling \\
\hline 46 & D0ELH5 & Peptidyl-prolyl cis-trans isomerase & C. sinensis & 10 & 209 & 0.31 & 8.46 & 18310 & cell \\
\hline 47 & K3Z5U9 & Uncharacterized protein & S. italica & 31 & 465 & 0.31 & 6.47 & 49826 & photosynthesis \\
\hline 48 & D6PAY2 & Peptidyl-prolyl cis-trans isomerase & V. hybrid & 15 & 217 & 0.31 & 8.46 & 18582 & cell \\
\hline 49 & O50036 & Heat shock 70 protein & S. oleracea & 41 & 393 & 0.30 & 4.67 & 76266 & stress \\
\hline 50 & B4FL17 & Translationally-controlled tumor protein & Z. mays & 16 & 123 & 0.30 & 5.30 & 18773 & development \\
\hline 51 & O81830 & Putative uncharacterized protein AT4g27270 & A. thaliana & 9 & 223 & 0.30 & 6.79 & 22355 & lipid metabolism \\
\hline 52 & K3YR06 & Uncharacterized protein & S. italica & 28 & 828 & 0.30 & 6.16 & 64225 & protein \\
\hline 53 & $\mathrm{O} 48903$ & Malate dehydrogenase & M. sativa & 31 & 780 & 0.30 & 8.29 & 38397 & gluconeogenesis \\
\hline 54 & A2V880 & G protein B.-subunit-like protein & N. tabacum & 5 & 91 & 0.30 & 7.09 & 16100 & signalling \\
\hline 55 & $\mathrm{~F} 2 \mathrm{E} 4 \mathrm{C} 2$ & Predicted protein & H. vulgare & 60 & 838 & 0.29 & 5.25 & 72202 & stress \\
\hline 56 & G5DXP1 & Fructose-bisphosphate aldolase & S. latifolia & 14 & 410 & 0.29 & 6.16 & 42771 & photosynthesis \\
\hline 57 & Q9SXX4 & Fructose-bisphosphate aldolase & N. paniculata & 16 & 376 & 0.29 & 7.33 & 42832 & photosynthesis \\
\hline 58 & K3Z913 & Uncharacterized protein & S. italica & 16 & 316 & 0.28 & 9.47 & 26793 & cell \\
\hline 59 & A9PD17 & Predicted protein & P. trichocarpa & 39 & 1072 & 0.28 & 5.82 & 47103 & protein \\
\hline 60 & A4UMS1 & Myo-inositol-1-phosphate synthase isoform 1 & $X . v i s c o s a$ & 27 & 479 & 0.28 & 5.41 & 56899 & minor $\mathrm{CHO}$ metabolism \\
\hline 61 & E4MXI2 & mRNA, clone: RTFL01-39-D20 & T. halophila & 34 & 348 & 0.28 & 5.21 & 73890 & stress \\
\hline 62 & A7WMM1 & Alanine:glyoxylate aminotransferase & L. caprifolium & 6 & 38 & 0.28 & 8.43 & 13805 & amino acid metabolism \\
\hline 63 & Q8GZD5 & Xyloglucan endotransglycosylase & P. tremula & 39 & 430 & 0.27 & 7.75 & 34481 & cell wall \\
\hline
\end{tabular}




\begin{tabular}{|c|c|c|c|c|c|c|c|c|c|}
\hline 64 & Q8MCY2 & Ribulose bisphosphate carboxylase large chain & V. baccifera & 36 & 346 & 0.27 & 6.64 & 48739 & photosynthesis \\
\hline 65 & K3XFR6 & Uncharacterized protein & S. italica & 27 & 337 & 0.27 & 7.46 & 66448 & TCA \\
\hline 66 & B6TWN7 & Elongation factor 1-alpha & Z. mays & 21 & 72 & 0.26 & 9.07 & 49594 & protein \\
\hline 67 & B3TLQ5 & 60S ribosomal protein L11 & E. guineensis & 6 & 154 & 0.26 & 9.92 & 20950 & protein \\
\hline 68 & B4G0K4 & Phosphoglycerate kinase & Z. mays & 38 & 569 & 0.26 & 5.87 & 42470 & glycolysis \\
\hline 69 & K3XXC5 & Uncharacterized protein & S. italica & 59 & 577 & 0.26 & 7.06 & 42409 & glycolysis \\
\hline 70 & Q069K2 & 5-methyltetrahydropteroyltriglutamate--homocysteine methyltransferase & N. suaveolens & 72 & 857 & 0.26 & 6.62 & 85060 & amino acid metabolism \\
\hline 71 & Q84RD8 & Adenosylhomocysteinase & M. truncatula & 37 & 427 & 0.26 & 6.01 & 53790 & amino acid metabolism \\
\hline 72 & B6TPE4 & Glutamate-1-semialdehyde 2,1-aminomutase & Z. mays & 16 & 163 & 0.26 & 6.55 & 50223 & tetrapyrrole synthesis \\
\hline 73 & Q06Z74 & Fibrillin 4 & C. canephora & 11 & 288 & 0.26 & 8.88 & 32064 & cell \\
\hline 74 & A8MRE8 & Triosephosphate isomerase & A. thaliana & 15 & 185 & 0.26 & 7.49 & 32458 & photosynthesis \\
\hline 75 & E4MXI1 & mRNA, clone: RTFL01-39-B07 & T. halophila & 20 & 348 & 0.26 & 6.98 & 48063 & photosynthesis \\
\hline 76 & Q7XJH9 & Transaldolase & S. lycopersicum & 17 & 198 & 0.25 & 5.96 & 48062 & OPP \\
\hline 77 & D3GQL2 & Aconitate hydratase 2 & C. clementina & 80 & 828 & 0.25 & 6.54 & 98475 & TCA \\
\hline 78 & D3GQL1 & Aconitate hydratase 3 & C. clementina & 64 & 936 & 0.24 & 6.30 & 98669 & TCA \\
\hline 79 & Q6VWJ5 & Fructokinase 3 & S. lycopersicum & 14 & 221 & 0.24 & 5.80 & 41803 & major $\mathrm{CHO}$ metabolism \\
\hline 80 & K3XIT0 & Uncharacterized protein & S. italica & 16 & 445 & 0.24 & 8.78 & 41915 & photosynthesis \\
\hline 81 & Q9AXR6 & ATP:citrate lyase & C. аппиит & 26 & 360 & 0.24 & 7.39 & 66376 & TCA \\
\hline 82 & Q7XAE2 & Putative fructokinase 2 & P. integrifolia & 33 & 477 & 0.23 & 5.35 & 35181 & major $\mathrm{CHO}$ metabolism \\
\hline 83 & A8J195 & Small rab-related GTPase & C. reinhardtii & 5 & 124 & 0.23 & 7.46 & 23825 & signalling \\
\hline 84 & E1ZKK0 & Putative uncharacterized protein & C. variabilis & 9 & 167 & 0.23 & 7.20 & 35948 & photosynthesis \\
\hline 85 & Q9SRV5 & 5-methyltetrahydropteroyltriglutamate--homocysteine methyltransferase & A. thaliana & 83 & 1029 & 0.23 & 6.51 & 84873 & amino acid metabolism \\
\hline 86 & K3XEI1 & Uncharacterized protein & S. italica & 50 & 561 & 0.22 & 6.27 & 94876 & protein \\
\hline 87 & Q5ZF83 & Putative uncharacterized protein & P. major & 15 & 120 & 0.22 & 7.28 & 25390 & cell wall \\
\hline 88 & G1DVA7 & Non-specific lipid-transfer protein & H. annииs & 4 & 42 & 0.22 & 8.41 & 12193 & lipid metabolism \\
\hline 89 & Q6QND3 & Putative pyridoxine biosynthesis protein isoform A & N. tabacum & 6 & 122 & 0.21 & 6.32 & 33353 & co-factor and vitamine metabolism \\
\hline 90 & A1YUL9 & Importin subunit alpha & N. benthamiana & 16 & 345 & 0.21 & 5.38 & 59114 & protein \\
\hline 91 & B6TGL7 & Enolase & Z. mays & 14 & 502 & 0.21 & 5.10 & 50512 & glycolysis \\
\hline 92 & G9MA91 & Fructose-bisphosphate aldolase & L. grandiflorum & 8 & 266 & 0.21 & 6.23 & 37690 & glycolysis \\
\hline 93 & Q645M9 & Glyoxisomal malate dehydrogenase & S. lycopersicum & 17 & 395 & 0.21 & 6.28 & 38007 & gluconeogenesis \\
\hline 94 & A2Q5W0 & Alpha-tubulin & M. truncatula & 18 & 384 & 0.21 & 7.94 & 50457 & cell \\
\hline 95 & E4MVY3 & mRNA, clone: RTFL01-01-M18 & T. halophila & 43 & 764 & 0.21 & 6.25 & 94734 & protein \\
\hline
\end{tabular}




\begin{tabular}{|c|c|c|c|c|c|c|c|c|c|}
\hline 96 & F2D690 & Predicted protein & H. vulgare & 2 & 156 & 0.21 & 4.37 & 12678 & protein \\
\hline 97 & O04975 & Fructose-bisphosphate aldolase & M. crystalL. & 12 & 182 & 0.21 & 6.92 & 38362 & glycolysis \\
\hline 98 & F2EE28 & Predicted protein & H. vulgare & 24 & 401 & 0.21 & 5.55 & 61280 & protein \\
\hline 99 & A1BLP6 & Thioredoxin & M. truncatula & 11 & 69 & 0.20 & 6.16 & 12904 & redox \\
\hline 100 & Q9ATF4 & Ribosomal protein L33 & C. sativa & 3 & 116 & 0.20 & 10.58 & 12831 & protein \\
\hline 101 & D0EJY9 & Molecular chaperone Hsp90-3 & N. benthamiana & 31 & 582 & 0.20 & 5.02 & 80638 & stress \\
\hline 102 & Q8LG68 & UDP-glucose 6-dehydrogenase & A. thaliana & 19 & 393 & 0.20 & 6.18 & 53591 & stress \\
\hline 103 & F2DAU4 & Predicted protein & H. vulgare & 35 & 398 & 0.20 & 7.56 & 62696 & protein \\
\hline 104 & Q42425 & Aspartate aminotransferase & P. miliaceum & 17 & 124 & 0.19 & 8.48 & 50477 & amino acid metabolism \\
\hline 105 & F2DTB2 & Triosephosphate isomerase & H. vulgare & 19 & 104 & 0.19 & 6.80 & 32679 & photosynthesis \\
\hline 106 & A1BQW9 & Transketolase & N. attenuata & 28 & 170 & 0.19 & 7.42 & 32653 & OPP \\
\hline 107 & H2BBB1 & ATP synthase subunit alpha, chloroplastic & P. diguetii & 14 & 316 & 0.19 & 5.74 & 55710 & photosynthesis \\
\hline 108 & Q34691 & ATP synthase subunit alpha & H. annuиs & 22 & 314 & 0.19 & 6.60 & 55766 & mitoETC \\
\hline 109 & B5LAU5 & DH putative B.-hydroxyacyl-ACP dehydratase & C. аппиит & 8 & 78 & 0.18 & 9.29 & 24080 & lipid metabolism \\
\hline 110 & K3YG24 & Uncharacterized protein & S. italica & 58 & 561 & 0.18 & 7.31 & 107135 & TCA \\
\hline 111 & Q9FE12 & Peroxiredoxin & P. vulgaris & 7 & 223 & 0.18 & 5.33 & 28776 & redox \\
\hline 112 & Q0W9E2 & B. chaperonin 60 & S. commersonii & 17 & 478 & 0.18 & 7.27 & 63333 & protein \\
\hline 113 & K3YR95 & Uncharacterized protein & S. italica & 26 & 322 & 0.18 & 7.65 & 58776 & protein \\
\hline 114 & H9NIE1 & Cell division cycle protein 48 & C. sinensis & 29 & 551 & 0.17 & 5.29 & 90644 & cell \\
\hline 115 & O04946 & Enoyl-ACP reductase & N. tabacum & 12 & 159 & 0.17 & 8.78 & 41925 & lipid metabolism \\
\hline 116 & B4FPK8 & Cytochrome b5 & Z. mays & 4 & 101 & 0.17 & 5.30 & 14958 & redox \\
\hline 117 & F2CQ27 & Predicted protein & H. vulgare & 11 & 54 & 0.17 & 6.66 & 25460 & signalling \\
\hline 118 & K4A7I4 & Uncharacterized protein & S. italica & 19 & 477 & 0.17 & 4.84 & 61666 & photosynthesis \\
\hline 119 & A8MQR4 & $60 \mathrm{~S}$ acidic ribosomal protein $\mathrm{P} 0-2$ & A. thaliana & 10 & 170 & 0.17 & 10.17 & 30656 & protein \\
\hline 120 & F2D448 & Predicted protein & H. vulgare & 11 & 127 & 0.17 & 5.40 & 30607 & protein \\
\hline 121 & A9Z0Q0 & Catalase & P. ginseng & 20 & 388 & 0.17 & 7.18 & 57016 & redox \\
\hline 122 & Q4H1G2 & Methionine synthase & B. vulgaris & 89 & 1267 & 0.17 & 6.46 & 88546 & amino acid metabolism \\
\hline 123 & B6T6Q8 & Alcohol dehydrogenase class 3 & Z. mays & 8 & 143 & 0.17 & 7.02 & 41639 & misc \\
\hline 124 & A9ZMZ5 & Acetyl-CoA C-acetyltransferase & H. brasiliensis & 29 & 789 & 0.17 & 6.68 & 42039 & secondary metabolism \\
\hline 125 & Q1KUX5 & 6-phosphogluconate dehydrogenase, decarboxylating & C. spinosa & 22 & 184 & 0.17 & 6.06 & 53777 & OPP \\
\hline 126 & F2EKH0 & Predicted protein & H. vulgare & 9 & 258 & 0.16 & 4.60 & 31771 & protein \\
\hline 127 & K3ZIK0 & Uncharacterized protein & S. italica & 17 & 474 & 0.16 & 6.61 & 47729 & photosynthesis \\
\hline
\end{tabular}




\begin{tabular}{|c|c|c|c|c|c|c|c|c|c|}
\hline 128 & B3TLL5 & 40S ribosomal protein S19 & E. guineensis & 13 & 58 & 0.16 & 10.04 & 16202 & protein \\
\hline 129 & C6T859 & B.-form rubisco activase & G. $\max$ & 21 & 571 & 0.16 & 7.24 & 48764 & photosynthesis \\
\hline 130 & A5JVC6 & Putative uncharacterized protein & B. campestris & 7 & 142 & 0.15 & 6.25 & 34259 & cell wall \\
\hline 131 & $\mathrm{O} 24511$ & Catalase & N. tabacum & 19 & 133 & 0.15 & 6.86 & 57225 & redox \\
\hline 132 & Q96350 & Catalase & B. napus & 13 & 152 & 0.15 & 7.81 & 57170 & redox \\
\hline 133 & F2DBE0 & Predicted protein & H. vulgare & 6 & 87 & 0.15 & 8.22 & 34595 & TCA \\
\hline 134 & Q8LBY1 & Hydroxymethyltransferase & A. thaliana & 19 & 457 & 0.15 & 7.02 & 52129 & C1-metabolism \\
\hline 135 & A9CM22 & Voltage-dependent anion channel & N. tabacum & 5 & 101 & 0.15 & 8.32 & 29694 & transport \\
\hline 136 & F2E397 & Predicted protein & H. vulgare & 10 & 262 & 0.14 & 4.98 & 34933 & major $\mathrm{CHO}$ metabolism \\
\hline 137 & B9RZK1 & Major allergen Pru ar, putative & R. communis & 2 & 46 & 0.14 & 5.20 & 17294 & stress \\
\hline 138 & D2DMF5 & Chloroplast plastocyanin & N. benthamiana & 12 & 28 & 0.14 & 5.87 & 17202 & photosynthesis \\
\hline 139 & Q9SXR9 & LeArcA1 protein & S. lycopersicum & 6 & 54 & 0.14 & 7.12 & 36363 & signalling \\
\hline 140 & J7MPY1 & ATP synthase subunit alpha & M. domestica & 18 & 125 & 0.14 & 6.61 & 55600 & mitoETC \\
\hline 141 & D7LW57 & Ketol-acid reductoisomerase & A. lyrata & 11 & 300 & 0.13 & 6.80 & 64346 & amino acid metabolism \\
\hline 142 & D7UPN0 & SEC13 family protein & L. japonicus & 3 & 71 & 0.13 & 6.01 & 32940 & protein \\
\hline 143 & E4MX73 & mRNA, clone: RTFL01-10-N01 & T. halophila & 12 & 399 & 0.13 & 7.44 & 39684 & TCA \\
\hline 144 & K3XVH0 & Uncharacterized protein & S. italica & 35 & 313 & 0.13 & 5.83 & 80203 & photosynthesis \\
\hline 145 & C1M2W0 & Somatic embryogenesis cinnamyl alcohol dehydrogenase 1 & C. sativus & 11 & 203 & 0.13 & 6.62 & 39462 & secondary metabolism \\
\hline 146 & K3ZSX0 & Uncharacterized protein & S. italica & 6 & 120 & 0.13 & 7.64 & 53564 & amino acid metabolism \\
\hline 147 & E4MWY6 & Glucose-6-phosphate isomerase & T. halophila & 8 & 233 & 0.13 & 5.99 & 67312 & glycolysis \\
\hline 148 & B6SWT4 & Glucose-6-phosphate isomerase & Z. mays & 8 & 273 & 0.13 & 5.60 & 67980 & glycolysis \\
\hline 149 & F6H3T7 & Putative uncharacterized protein & V. vinifera & 16 & 85 & 0.12 & 6.11 & 40651 & not assigned \\
\hline 150 & E5F725 & Putative uncharacterized protein & E. parvulum & 6 & 255 & 0.12 & 7.11 & 42041 & cell wall \\
\hline 151 & D8L9Q5 & Fructose-bisphosphate aldolase & T. aestivum & 13 & 351 & 0.12 & 8.09 & 41564 & photosynthesis \\
\hline 152 & E4MWP5 & mRNA, clone: RTFL01-15-G22 & T. halophila & 20 & 58 & 0.12 & 6.16 & 35258 & photosynthesis \\
\hline 153 & K3ZU91 & Uncharacterized protein & S. italica & 6 & 71 & 0.12 & 6.81 & 42567 & protein \\
\hline 154 & K3XFY9 & Uncharacterized protein & S. italica & 14 & 175 & 0.12 & 8.70 & 64010 & cell \\
\hline 155 & Q9SQI8 & 2-oxoacid dehydrogenases acyltransferase family protein & A. thaliana & 9 & 154 & 0.12 & 8.37 & 50106 & lipid metabolism \\
\hline 156 & E4MXR5 & mRNA, clone: RTFL01-40-M04 & T. halophila & 24 & 373 & 0.12 & 5.90 & 73489 & stress \\
\hline 157 & O78327 & Transketolase 1 & С. аппиит & 38 & 338 & 0.11 & 6.62 & 80398 & OPP \\
\hline 158 & E5GBD5 & $60 \mathrm{~S}$ ribosomal protein 19 & C. melo & 3 & 72 & 0.11 & 9.45 & 21994 & protein \\
\hline 159 & D2XNF3 & Chaperone protein DnaK & P. patens & 13 & 136 & 0.11 & 5.50 & 75674 & stress \\
\hline
\end{tabular}




\begin{tabular}{|c|c|c|c|c|c|c|c|c|c|}
\hline 160 & Q0MYQ7 & Germin-like protein 2 & V. vinifera & 3 & 157 & 0.11 & 9.58 & 22832 & stress \\
\hline 161 & K3XZE3 & Uncharacterized protein & S. italica & 3 & 147 & 0.11 & 5.27 & 23153 & protein \\
\hline 162 & K3XZA2 & Uncharacterized protein & S. italica & 13 & 178 & 0.11 & 5.96 & 23221 & cell \\
\hline 163 & $\mathrm{O} 22143$ & At2g45290/F4L23.20 & A. thaliana & 14 & 218 & 0.11 & 8.41 & 69264 & photosynthesis \\
\hline 164 & $\mathrm{O} 23963$ & Elongation factor $\mathrm{Tu}$ & G. $\max$ & 21 & 190 & 0.11 & 5.14 & 38044 & protein \\
\hline 165 & C1IC54 & Cinnamyl alcohol dehydrogenase & G. hirsutum & 9 & 48 & 0.10 & 6.25 & 39574 & secondary metabolism \\
\hline 166 & E4MY36 & mRNA, clone: RTFL01-37-B16 & T. halophila & 10 & 80 & 0.10 & 6.25 & 63555 & glycolysis \\
\hline 167 & F2CQP8 & Predicted protein & H. vulgare & 9 & 61 & 0.10 & 5.49 & 32811 & biodegradation of xenobiotics. \\
\hline 168 & O04428 & Putative uncharacterized protein & C. paradisi & 15 & 236 & 0.10 & 5.59 & 32737 & biodegradation of xenobiotics. \\
\hline 169 & E5LCX1 & C14 cysteine protease & S. demissum & 6 & 195 & 0.10 & 8.67 & 23974 & protein \\
\hline 170 & B6SVI5 & Putative uncharacterized protein & Z. mays & 8 & 32 & 0.10 & 4.98 & 24337 & not assigned \\
\hline 171 & E5GBJ6 & Alcohol dehydrogenase & C. melo & 3 & 131 & 0.10 & 8.47 & 40658 & misc \\
\hline 172 & B6UDL5 & Hydroxymethylbutenyl 4-diphosphate synthase & Z. mays & 29 & 412 & 0.10 & 5.97 & 82691 & secondary metabolism \\
\hline 173 & B3TLP3 & Translation elongation factor EF-1 B. chain & E. guineensis & 6 & 179 & 0.10 & 4.69 & 24523 & protein \\
\hline 174 & E4MWC3 & Aspartate aminotransferase & T. halophila & 8 & 66 & 0.10 & 9.25 & 49678 & amino acid metabolism \\
\hline 175 & H6VUT7 & Translation elongation factor & A. mongolicus & 2 & 111 & 0.10 & 6.89 & 25300 & protein \\
\hline 176 & А9РАK9 & Putative uncharacterized protein & P. trichocarpa & 11 & 198 & 0.10 & 4.63 & 50623 & amino acid metabolism \\
\hline 177 & Q39860 & GTPase & G. $\max$ & 7 & 47 & 0.10 & 5.34 & 24700 & signalling \\
\hline 178 & Q706C9 & GDP dissociation inhibitor & M. truncatula & 5 & 84 & 0.10 & 5.88 & 50211 & signalling \\
\hline 179 & A3QVW8 & Glucose-6-phosphate isomerase & M. polymorpha & 10 & 68 & 0.10 & 7.18 & 68171 & glycolysis \\
\hline 180 & F2DLC2 & Proteasome subunit alpha type & H. vulgare & 4 & 186 & 0.09 & 8.05 & 26087 & protein \\
\hline 181 & F2DP49 & Predicted protein & H. vulgare & 12 & 116 & 0.09 & 4.68 & 25913 & hormone metabolism \\
\hline 182 & F2CXV7 & Predicted protein & H. vulgare & 2 & 106 & 0.09 & 8.63 & 25809 & protein \\
\hline 183 & B6TGG2 & 2,3-bisphosphoglycerate-independent phosphoglycerate mutase & Z. mays & 15 & 174 & 0.09 & 5.53 & 60811 & glycolysis \\
\hline 184 & G8HAB2 & PLP-dependent aminotransferase & P. somniferum & 8 & 144 & 0.09 & 5.63 & 53501 & amino acid metabolism \\
\hline 185 & F2CW31 & Predicted protein & H. vulgare & 14 & 205 & 0.09 & 6.21 & 63075 & glycolysis \\
\hline 186 & B5LAT6 & Dihydrolipoyl dehydrogenase & C. аппиит & 5 & 36 & 0.09 & 6.86 & 54133 & TCA \\
\hline 187 & F2D3S8 & Serine hydroxymethyltransferase & H. vulgare & 20 & 77 & 0.09 & 8.21 & 56215 & C1-metabolism \\
\hline 188 & Q93WQ1 & Dihydrolipoyl dehydrogenase & B. gymnorhiza & 6 & 108 & 0.09 & 7.17 & 54389 & TCA \\
\hline 189 & B5LAV3 & Putative isopropylmalate synthase & C. аппиит & 5 & 119 & 0.09 & 6.54 & 66084 & amino acid metabolism \\
\hline 190 & A9PE52 & Gamma class glutathione transferase EF1Bgamma2 & P. trichocarpa & 7 & 141 & 0.08 & 6.04 & 48127 & protein \\
\hline 191 & K3ZI40 & Uncharacterized protein & S. italica & 6 & 90 & 0.08 & 11.14 & 56750 & redox \\
\hline
\end{tabular}




\begin{tabular}{|c|c|c|c|c|c|c|c|c|c|}
\hline 192 & F2D9E0 & Predicted protein & H. vulgare & 4 & 110 & 0.08 & 5.01 & 28037 & protein \\
\hline 193 & Q5GAQ3 & Putative alanine aminotransferase & Z. mays & 11 & 163 & 0.08 & 5.64 & 57003 & amino acid metabolism \\
\hline 194 & K3XHU5 & Uncharacterized protein & S. italica & 15 & 228 & 0.08 & 6.43 & 48590 & metal handling \\
\hline 195 & K4A6V8 & Uncharacterized protein & S. italica & 13 & 138 & 0.08 & 7.03 & 70549 & glycolysis \\
\hline 196 & Q8L8H6 & Phytoene desaturase & T. erecta & 4 & 57 & 0.08 & 6.70 & 38216 & secondary metabolism \\
\hline 197 & K3Z3M1 & Uncharacterized protein & S. italica & 14 & 247 & 0.08 & 7.06 & 102144 & protein \\
\hline 198 & K3ZW60 & Uncharacterized protein & S. italica & 4 & 28 & 0.08 & 5.40 & 29913 & protein \\
\hline 199 & H9A1W2 & Acyl-activating enzyme 10 & C. sativa & 9 & 150 & 0.08 & 6.49 & 62013 & lipid metabolism \\
\hline 200 & B6TNX7 & Proteasome subunit alpha type & Z. mays & 5 & 147 & 0.08 & 9.76 & 30582 & protein \\
\hline 201 & K3XWI6 & Uncharacterized protein & S. italica & 10 & 162 & 0.07 & 6.60 & 55489 & glycolysis \\
\hline 202 & B0M1B8 & Citrate synthase & G. $\max$ & 4 & 55 & 0.07 & 8.09 & 54427 & gluconeogenesis \\
\hline 203 & I0J3D8 & Proteasome subunit B. type & A. halleri & 2 & 111 & 0.07 & 6.06 & 33367 & protein \\
\hline 204 & K3XKD1 & Uncharacterized protein & S. italica & 3 & 99 & 0.07 & 6.76 & 34429 & protein \\
\hline 205 & K3Z5E2 & Uncharacterized protein & S. italica & 5 & 110 & 0.07 & 8.09 & 56837 & glycolysis \\
\hline 206 & K3ZR86 & Uncharacterized protein & S. italica & 6 & 35 & 0.07 & 9.01 & 74682 & fermentation \\
\hline 207 & Q58H58 & Chloroplast photosynthetic oxygen-evolving protein $33 \mathrm{kDa}$ subunit & N. benthamiana & 3 & 34 & 0.07 & 5.66 & 35374 & photosynthesis \\
\hline 208 & Q39986 & Cysteine proteinase & Hemerocallis sp. & 4 & 155 & 0.06 & 8.18 & 40006 & protein \\
\hline 209 & E2FYC3 & Caffeic acid O-methyltransferase & C. sinensis & 2 & 80 & 0.06 & 6.05 & 40149 & secondary metabolism \\
\hline 210 & B9RI89 & Serine-threonine protein kinase, plant-type, putative & R. communis & 4 & 107 & 0.06 & 5.50 & 40375 & not assigned \\
\hline 211 & K4A8Y9 & Uncharacterized protein & S. italica & 4 & 192 & 0.06 & 7.61 & 53531 & TCA \\
\hline 212 & Q110X5 & Pyruvate kinase & C. аппиит & 10 & 44 & 0.06 & 6.84 & 56114 & glycolysis \\
\hline 213 & B4FYM0 & Acetyl-CoA acetyltransferase, cytosolic 2 & Z. mays & 7 & 205 & 0.06 & 4.88 & 43450 & secondary metabolism \\
\hline 214 & A8I7T1 & Heat shock protein $90 \mathrm{~B}$ & C. reinhardtii & 9 & 203 & 0.06 & 7.66 & 87547 & stress \\
\hline 215 & B9HJ23 & Predicted protein & P. trichocarpa & 12 & 179 & 0.05 & 6.54 & 101700 & misc \\
\hline 216 & Q1KUM7 & Putative uncharacterized protein & C. spinosa & 5 & 110 & 0.05 & 6.62 & 59525 & protein \\
\hline 217 & A9P9A2 & Putative uncharacterized protein & P. trichocarpa & 14 & 163 & 0.05 & 9.36 & 46272 & RNA \\
\hline 218 & F2DLJ7 & Predicted protein & H. vulgare & 2 & 143 & 0.05 & 7.77 & 44423 & hormone metabolism \\
\hline 219 & Q42737 & NADP-malate dehydrogenase & F. trinervia & 2 & 79 & 0.05 & 6.44 & 46155 & TCA \\
\hline 220 & K3XIH5 & Uncharacterized protein & S. italica & 4 & 28 & 0.05 & 6.46 & 44308 & secondary metabolism \\
\hline 221 & E4MVQ7 & mRNA, clone: RTFL01-03-C18 & T. halophila & 7 & 125 & 0.05 & 6.60 & 46631 & redox \\
\hline 222 & Q66PF9 & Monodehydroascorbate reductase I & P. sativum & 11 & 96 & 0.05 & 6.11 & 47436 & redox \\
\hline 223 & Q8LGJ6 & Phosphoglycerate dehydrogenase-like protein & A. thaliana & 6 & 116 & 0.05 & 6.76 & 63556 & amino acid metabolism \\
\hline
\end{tabular}




\begin{tabular}{|c|c|c|c|c|c|c|c|c|c|}
\hline 224 & G8HAB1 & PLP-dependent aminotransferase & P. somniferum & 4 & 78 & 0.05 & 7.87 & 50587 & secondary metabolism \\
\hline 225 & Q93YG1 & Monodehydroascorbate reductase & M. crystalL. & 15 & 115 & 0.05 & 7.15 & 51944 & redox \\
\hline 226 & F2DDG9 & Predicted protein & H. vulgare & 2 & 38 & 0.05 & 5.66 & 52441 & cell \\
\hline 227 & A9RZ64 & Predicted protein & P. patens & 2 & 154 & 0.05 & 7.09 & 50746 & amino acid metabolism \\
\hline 228 & F2DAD8 & Predicted protein & H. vulgare & 6 & 54 & 0.05 & 6.81 & 50084 & amino acid metabolism \\
\hline 229 & A0FKE6 & Chloroplast threonine deaminase 1 & S. lycopersicum & 9 & 173 & 0.05 & 6.37 & 66653 & amino acid metabolism \\
\hline 230 & F2DAP7 & Malic enzyme & H. vulgare & 6 & 170 & 0.05 & 5.78 & 69436 & TCA \\
\hline 231 & K3YRV8 & Uncharacterized protein & S. italica & 4 & 53 & 0.04 & 6.92 & 55802 & TCA \\
\hline 232 & С0Р9J6 & Aminoaldehyde dehydrogenase 1 & Z. mays & 4 & 49 & 0.04 & 5.45 & 55733 & secondary metabolism \\
\hline 233 & K4A938 & Uncharacterized protein & S. italica & 2 & 55 & 0.04 & 7.27 & 53547 & nucleotide metabolism \\
\hline 234 & Q9S768 & Alanine aminotransferase & O. sativa & 6 & 207 & 0.04 & 6.79 & 53130 & amino acid metabolism \\
\hline 235 & Q6XXZ2 & Gamma-glutamylcysteine synthetase & L. japonicus & 9 & 184 & 0.04 & 6.67 & 56232 & redox \\
\hline 236 & Q9FRW7 & Aspartic proteinase 3 & N. alata & 2 & 85 & 0.04 & 5.22 & 55666 & protein \\
\hline 237 & B6THZ8 & Threonine synthase & Z. mays & 2 & 48 & 0.04 & 6.65 & 58091 & amino acid metabolism \\
\hline 238 & Q6L619 & B.-galactosidase & R. sativus & 6 & 101 & 0.04 & 8.38 & 93664 & misc \\
\hline 239 & Q9SA89 & FAD-binding and BBE domain-containing protein & A. thaliana & 8 & 38 & 0.04 & 6.76 & 60060 & not assigned \\
\hline 240 & F2Z9R1 & Glucose-6-phosphate 1-dehydrogenase & N. benthamiana & 12 & 68 & 0.04 & 6.83 & 58894 & OPP \\
\hline 241 & B6T715 & Aldehyde dehydrogenase & Z. mays & 3 & 80 & 0.04 & 6.49 & 59108 & fermentation \\
\hline 242 & E1ZNM5 & Putative uncharacterized protein & C. variabilis & 8 & 47 & 0.04 & 8.60 & 60332 & amino acid metabolism \\
\hline 243 & B5BLW2 & Carotenoid cleavage dioxygenase 1 & M. truncatula & 5 & 58 & 0.04 & 6.79 & 61063 & secondary metabolism \\
\hline 244 & Q9ZVV4 & At1g55570/T5A14_1 & A. thaliana & 4 & 32 & 0.04 & 8.91 & 62687 & misc \\
\hline 245 & Q38766 & Victorin binding protein & A. sativa & 5 & 57 & 0.03 & 6.96 & 112298 & photosynthesis \\
\hline 246 & $\mathrm{~J} 9 \mathrm{Z} 414$ & B.-D-glucan exohydolase & E. guineensis & 4 & 111 & 0.03 & 5.03 & 69462 & cell wall \\
\hline 247 & B6TLT1 & Serine/threonine-protein phosphatase $2 \mathrm{~A} 65 \mathrm{kDa}$ regulatory subunit $\mathrm{AB}$. isoform & Z. mays & 4 & 43 & 0.03 & 7.88 & 66318 & protein \\
\hline 248 & K3YKV0 & Uncharacterized protein & S. italica & 8 & 117 & 0.03 & 5.54 & 87462 & cell \\
\hline 249 & B2KNE6 & Phospholipase D & H. annuиs & 8 & 142 & 0.02 & 6.16 & 92315 & lipid metabolism \\
\hline 250 & Q9XFX7 & Phospholipase D & C. plantagineum & 7 & 35 & 0.02 & 5.52 & 92228 & lipid metabolism \\
\hline 251 & A5AW47 & Profilin & $V$. vinifera & 17 & 71 & 0.02 & 5.66 & 92852 & cell \\
\hline 252 & F2CRM6 & Predicted protein & H. vulgare & 4 & 97 & 0.02 & 6.28 & 121394 & protein \\
\hline 253 & E1Z839 & Putative uncharacterized protein & C. variabilis & 11 & 38 & 0.02 & 5.52 & 111491 & DNA \\
\hline 254 & Q9ARB0 & Nbi-D protein & L. usitatissimum & 19 & 39 & 0.02 & 6.93 & 127688 & stress \\
\hline 255 & E4MXV2 & mRNA, clone: RTFL01-41-F15 & T. halophila & 10 & 90 & - & 6.09 & 64768 & secondary metabolism \\
\hline
\end{tabular}




$37 \quad 6.64$

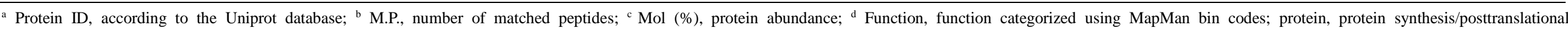

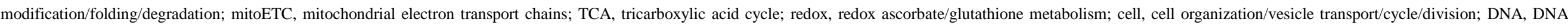
synthesis/repair; OPP, oxidative pentose phosphate; RNA, RNA processing/regulation of transcription; and misc, miscellaneous 
Supplemental Table 3. Proteins Identified in L. japonica Immature Flower Buds at Fraction of Elution of $\mathrm{pH} 7$ using Combinatorial Peptide Ligand Libraries Fractionation.

\begin{tabular}{|c|c|c|c|c|c|c|c|c|c|}
\hline No. & Protein ID ${ }^{a}$ & Description & Species & M.P. ${ }^{b}$ & Score & $\operatorname{Mol}(\%)^{\mathrm{c}}$ & $\mathrm{pI}$ & Mass (Da) & Function $^{\mathrm{d}}$ \\
\hline 1 & C4B8E5 & Glyceraldehyde-3-phosphate dehydrogenase & T. gesneriana & 102 & 3465 & 1.49 & 7.96 & 20964 & glycolysis \\
\hline 2 & H1ZY49 & Translation elongation factor 1-alpha & L. maackii & 42 & 459 & 1.35 & 5.00 & 11619 & protein \\
\hline 3 & E1ZL24 & Putative uncharacterized protein & C. variabilis & 39 & 398 & 1.19 & 6.93 & 20995 & protein \\
\hline 4 & B5LBN4 & Ribulose bisphosphate carboxylase large chain & B. alleghaniensis & 50 & 370 & 0.99 & 6.05 & 22478 & photosynthesis \\
\hline 5 & B3TLP4 & Ribosomal protein L30 & E. guineensis & 9 & 163 & 0.98 & 9.72 & 12451 & protein \\
\hline 6 & Q9SAQ0 & ATP synthase subunit beta & N. sylvestris & 74 & 1333 & 0.87 & 6.06 & 59597 & mitoETC \\
\hline 7 & Q3LGW4 & Ribulose-1,5-bisphosphate carboxylase/oxygenase large subunit & F. benjamina & 10 & 181 & 0.83 & 4.67 & 9052 & photosynthesis \\
\hline 8 & Q6PKU2 & Putative glyceraldehyde-3-phosphate dehydrogenase & O. minor & 57 & 1615 & 0.82 & 7.09 & 22122 & glycolysis \\
\hline 9 & F2D884 & Predicted protein & H. vulgare & 96 & 1453 & 0.76 & 5.19 & 71476 & stress \\
\hline 10 & A7KQH1 & Beta-tubulin & E. grandis & 69 & 882 & 0.74 & 4.86 & 51031 & cell \\
\hline 11 & Q9FX54 & At1g13440/F13B4_8 & A. thaliana & 108 & 3717 & 0.71 & 7.18 & 37004 & glycolysis \\
\hline 12 & Q40463 & NTGB2 & N. tabacum & 8 & 169 & 0.68 & 6.19 & 15827 & signalling \\
\hline 13 & B0M1B1 & Peroxisomal glycolate oxidase & G. $\max$ & 33 & 275 & 0.66 & 9.13 & 40825 & photosynthesis \\
\hline 14 & Q5KTY2 & Ribulose-1,5-bisphosphate carboxylase large subunit & A. sutepensis & 94 & 853 & 0.64 & 6.92 & 51143 & photosynthesis \\
\hline 15 & J3RTS9 & Glyceraldehyde-3-phosphate dehydrogenase & C. pentagona & 122 & 3475 & 0.62 & 7.23 & 32382 & glycolysis \\
\hline 16 & Q9FEM4 & Catalase & B. pendula & 26 & 331 & 0.61 & 5.48 & 17247 & redox \\
\hline 17 & J9QAL9 & Ribosomal protein L2 & A. corniculatum & 15 & 245 & 0.59 & 10.23 & 21703 & protein \\
\hline 18 & A7KQH2 & Beta-tubulin & E. grandis & 63 & 782 & 0.59 & 4.84 & 50255 & cell \\
\hline 19 & A8MSE8 & Elongation factor 1-alpha & A. thaliana & 44 & 490 & 0.58 & 8.34 & 44670 & protein \\
\hline 20 & K3Z4G6 & Uncharacterized protein & S. italica & 53 & 836 & 0.57 & 5.25 & 71367 & stress \\
\hline 21 & A9PD17 & Predicted protein & P. trichocarpa & 52 & 1182 & 0.55 & 5.82 & 47103 & protein \\
\hline 22 & D2CJB9 & ATP synthase subunit beta & B. multifida & 28 & 685 & 0.54 & 5.05 & 51275 & photosynthesis \\
\hline 23 & Q5ZF83 & Putative uncharacterized protein & P. major & 24 & 357 & 0.53 & 7.28 & 25390 & cell wall \\
\hline 24 & Q08II7 & Heat shock protein 70 -like protein & L. sativa & 4 & 210 & 0.53 & 10.33 & 12539 & stress \\
\hline 25 & Q4H1G1 & Adenosylhomocysteinase & B. vulgaris & 50 & 543 & 0.53 & 6.19 & 54052 & amino acid metabolism \\
\hline 26 & Q9TKG9 & Ribulose 1,5-bisphosphate carboxylase large subunit & R. tomentosum & 108 & 1123 & 0.51 & 7.11 & 52772 & photosynthesis \\
\hline 27 & Q9TKH9 & Ribulose 1,5-bisphosphate carboxylase large subunit & H. albomarginata & 82 & 1057 & 0.50 & 6.37 & 51833 & photosynthesis \\
\hline 28 & Q8MCX1 & Ribulose-1,5-bisphosphate carboxylase large subunit & C. axillaris & 89 & 803 & 0.49 & 6.13 & 52386 & photosynthesis \\
\hline 29 & Q6H3X8 & $14-3-3$ protein isoform $16 \mathrm{R}$ & S. tuberosum & 20 & 209 & 0.45 & 4.78 & 29032 & signalling \\
\hline 30 & G1DVA7 & Non-specific lipid-transfer protein & H. аппииs & 9 & 49 & 0.44 & 8.41 & 12193 & lipid metabolism \\
\hline 31 & F2CT86 & Predicted protein & H. vulgare & 7 & 140 & 0.44 & 7.47 & 22104 & signalling \\
\hline
\end{tabular}




\begin{tabular}{|c|c|c|c|c|c|c|c|c|c|}
\hline 32 & B6EBD6 & Heat shock protein $90-2$ & G. $\max$ & 73 & 925 & 0.43 & 5.07 & 80392 & stress \\
\hline 33 & F2EG92 & Predicted protein & H. vulgar & 4 & 120 & 0.40 & 12.12 & 7602 & protein \\
\hline 34 & A2V880 & $\mathrm{G}$ protein beta-subunit-like protein & N. tabacum & 13 & 174 & 0.38 & 7.09 & 16100 & signalling \\
\hline 35 & B3TLL5 & 40S ribosomal protein S19 & E. guineensis & 19 & 58 & 0.38 & 10.04 & 16202 & protein \\
\hline 36 & K3Y9X5 & Uncharacterized protein & S. italica & 13 & 364 & 0.37 & 10.39 & 24963 & protein \\
\hline 37 & F2DAU4 & Predicted protein & H. vulgar & 31 & 459 & 0.37 & 7.56 & 62696 & protein \\
\hline 38 & F2CQQ3 & Predicted protein & H. vulgare & 15 & 569 & 0.37 & 5.16 & 54310 & transport \\
\hline 39 & $\mathrm{~F} 2 \mathrm{E} 4 \mathrm{C} 2$ & Predicted protein & H. vulgar & 34 & 510 & 0.36 & 5.25 & 72202 & stress \\
\hline 40 & K4A6U5 & Uncharacterized protein & S. italica & 29 & 511 & 0.36 & 5.34 & 71710 & stress \\
\hline 41 & Q9M6B3 & Malate dehydrogenase & $V$. vinifera & 18 & 335 & 0.36 & 8.62 & 37137 & TCA \\
\hline 42 & A0ZQA9 & ATP synthase subunit beta & P. baccatum & 32 & 414 & 0.35 & 5.40 & 52897 & photosynthesis \\
\hline 43 & B3TLQ5 & $60 \mathrm{~S}$ ribosomal protein $\mathrm{L} 11$ & E. guineensis & 10 & 180 & 0.34 & 9.92 & 20950 & protein \\
\hline 44 & K3XFR6 & Uncharacterized protein & S. italica & 31 & 709 & 0.34 & 7.46 & 66448 & TCA \\
\hline 45 & A8J597 & Ribosomal protein L12 & C. reinhardtii & 11 & 125 & 0.34 & 9.06 & 17822 & protein \\
\hline 46 & D0ELH5 & Peptidyl-prolyl cis-trans isomerase & C. sinensis & 10 & 223 & 0.33 & 8.46 & 18310 & cell \\
\hline 47 & K4A9V6 & Uncharacterized protein & S. italica & 33 & 425 & 0.32 & 5.10 & 50519 & cell \\
\hline 48 & B6T9G3 & Alpha-1,4-glucan-protein synthase 1 & Z. mays & 31 & 487 & 0.32 & 7.05 & 41278 & cell wall \\
\hline 49 & F2D861 & Predicted protein & H. vulgare & 15 & 471 & 0.32 & 10.43 & 15765 & protein \\
\hline 50 & F2CQ27 & Predicted protein & H. vulgare & 11 & 105 & 0.31 & 6.66 & 25460 & signalling \\
\hline 51 & G5DVX2 & Phosphoglycerate kinase & S. latifolia & 36 & 429 & 0.31 & 6.58 & 51352 & photosynthesis \\
\hline 52 & A9P8Q7 & Predicted protein & P. trichocarpa & 17 & 318 & 0.31 & 4.84 & 28824 & signalling \\
\hline 53 & $\mathrm{O} 48903$ & Malate dehydrogenase & M. sativa & 22 & 487 & 0.31 & 8.29 & 38397 & gluconeogenesis \\
\hline 54 & B4YYB3 & ST103-2 & T. halophila & 5 & 60 & 0.31 & 9.70 & 9286 & stress \\
\hline 55 & F2E3I7 & Glutamine synthetase & H. vulgare & 27 & 266 & 0.30 & 5.48 & 39331 & N-metabolism \\
\hline 56 & K3Z5U9 & Uncharacterized protein & S. italica & 23 & 249 & 0.30 & 6.47 & 49826 & photosynthesis \\
\hline 57 & K3Z913 & Uncharacterized protein & S. italica & 15 & 284 & 0.30 & 9.47 & 26793 & cell \\
\hline 58 & D7NHW9 & 2-phospho-D-glycerate hydrolase & P. trifoliata & 27 & 467 & 0.29 & 5.78 & 47986 & glycolysis \\
\hline 59 & B6TI65 & Fructose-bisphosphate aldolase & Z. mays & 13 & 176 & 0.28 & 7.83 & 41924 & photosynthesis \\
\hline 60 & $\mathrm{~K} 3 \mathrm{XXC5}$ & Uncharacterized protein & S. italica & 20 & 253 & 0.28 & 5.58 & 42409 & glycolysis \\
\hline 61 & E4MW44 & mRNA, clone: RTFL01-05-I15 & T. halophila & 20 & 307 & 0.28 & 7.06 & 42078 & cell \\
\hline 62 & D6PPS8 & AT3G02360-like protein & C. grandiflora & 9 & 256 & 0.28 & 9.22 & 21010 & OPP \\
\hline 63 & K4ACE3 & Uncharacterized protein & S. italica & 29 & 631 & 0.28 & 6.09 & 35803 & TCA \\
\hline
\end{tabular}




\begin{tabular}{|c|c|c|c|c|c|c|c|c|c|}
\hline 64 & Q1KUX5 & 6-phosphogluconate dehydrogenase, decarboxylating & C. spinosa & 27 & 309 & 0.28 & 6.06 & 53777 & OPP \\
\hline 65 & B6SJZ8 & Glyceraldehyde-3-phosphate dehydrogenase, cytosolic & Z. mays & 71 & 2121 & 0.27 & 6.96 & 36614 & glycolysis \\
\hline 66 & B2VQE0 & Methionine synthase & O. ramosa & 62 & 671 & 0.27 & 6.40 & 84862 & amino acid metabolism \\
\hline 67 & Q52UN0 & Peptidyl-prolyl cis-trans isomerase & C. sativus & 13 & 52 & 0.26 & 8.44 & 18340 & cell \\
\hline 68 & O81830 & Putative uncharacterized protein AT4g27270 & A. thaliana & 6 & 122 & 0.26 & 6.79 & 22355 & lipid metabolism \\
\hline 69 & B3H4P2 & Glyceraldehyde-3-phosphate dehydrogenase (NADP+) & A. thaliana & 10 & 80 & 0.26 & 7.77 & 37929 & photosynthesis \\
\hline 70 & A8MR50 & $60 \mathrm{~S}$ ribosomal protein $\mathrm{L} 34-1$ & A. thaliana & 2 & 107 & 0.26 & 11.84 & 11023 & cell wall \\
\hline 71 & Q7XTK9 & Catalase & P. persica & 41 & 662 & 0.26 & 7.12 & 57262 & redox \\
\hline 72 & B5TM95 & NADH dehydrogenase subunit 7 & A. thaliana & 10 & 202 & 0.26 & 7.25 & 45555 & mitoETC \\
\hline 73 & Q6T379 & Triosephosphate isomerase & S. chacoense & 8 & 131 & 0.25 & 5.99 & 27251 & glycolysis \\
\hline 74 & Q9AXR6 & ATP:citrate lyase & C. аппиит & 30 & 450 & 0.25 & 7.39 & 66376 & TCA \\
\hline 75 & F2E7L1 & Predicted protein & H. vulgare & 17 & 197 & 0.25 & 9.95 & 50919 & cell \\
\hline 76 & $\mathrm{~A} 2 \mathrm{PZC} 2$ & UDP-Glucose:protein transglucosylase & C. reinhardtii & 12 & 82 & 0.24 & 6.33 & 39846 & cell wall \\
\hline 77 & E4MXI2 & mRNA, clone: RTFL01-39-D20 & T. halophila & 31 & 573 & 0.23 & 5.21 & 73890 & stress \\
\hline 78 & Q645M9 & Glyoxisomal malate dehydrogenase & S. lycopersicum & 24 & 540 & 0.23 & 7.94 & 38007 & gluconeogenesis \\
\hline 79 & E4MVY3 & mRNA, clone: RTFL01-01-M18 & T. halophila & 43 & 852 & 0.22 & 6.25 & 94734 & protein \\
\hline 80 & K3YQW5 & Uncharacterized protein & S. italica & 13 & 369 & 0.22 & 5.39 & 68637 & transport \\
\hline 81 & A5JVC6 & Putative uncharacterized protein & B. campestris & 20 & 116 & 0.22 & 6.25 & 34259 & cell wall \\
\hline 82 & B6THG9 & $60 \mathrm{~S}$ ribosomal protein $\mathrm{L} 5-1$ & Z. mays & 18 & 341 & 0.22 & 9.32 & 34462 & protein \\
\hline 83 & K3ZTJ3 & Uncharacterized protein & S. italica & 14 & 269 & 0.22 & 8.94 & 47124 & photosynthesis \\
\hline 84 & Q0VYL5 & GASA & F. sylvatica & 2 & 44 & 0.22 & 9.03 & 12634 & hormone metabolism \\
\hline 85 & $\mathrm{O} 23254$ & Serine hydroxymethyltransferase & A. thaliana & 19 & 467 & 0.22 & 7.23 & 52141 & C1-metabolism \\
\hline 86 & $\mathrm{O} 24511$ & Catalase & N. tabacum & 29 & 369 & 0.22 & 6.86 & 57225 & redox \\
\hline 87 & A1BLP6 & Thioredoxin & M. truncatula & 9 & 88 & 0.22 & 6.16 & 12904 & redox \\
\hline 88 & K3XH89 & Uncharacterized protein & S. italica & 16 & 129 & 0.22 & 5.87 & 52978 & OPP \\
\hline 89 & Q6SKP4 & Ribosomal protein L3 & S. lycopersicum & 16 & 115 & 0.21 & 10.08 & 44836 & protein \\
\hline 90 & G5DWK9 & 3-dehydroquinate synthase & S. latifolia & 3 & 37 & 0.21 & 7.03 & 13075 & $\begin{array}{l}\text { co-factor and vitamine } \\
\text { metabolism }\end{array}$ \\
\hline 91 & A0EJL8 & GDP-D-mannose-3',5'-epimerase & M. glabra & 18 & 157 & 0.20 & 6.21 & 42927 & redox \\
\hline 92 & G5DXP1 & Fructose-bisphosphate aldolase & S. latifolia & 7 & 259 & 0.20 & 6.16 & 42771 & photosynthesis \\
\hline 93 & K4A7I4 & Uncharacterized protein & S. italica & 7 & 356 & 0.20 & 5.40 & 61666 & photosynthesis \\
\hline 94 & B5LAU9 & Glutamine synthetase & С. аппиит & 25 & 126 & 0.20 & 6.93 & 47926 & $\mathrm{~N}$-metabolism \\
\hline 95 & F2CQ90 & Predicted protein & H. vulgare & 2 & 52 & 0.20 & 7.12 & 14005 & protein \\
\hline
\end{tabular}




\begin{tabular}{|c|c|c|c|c|c|c|c|c|c|}
\hline 96 & B5LAU5 & DH putative beta-hydroxyacyl-ACP dehydratase & C. аппиит & 8 & 69 & 0.19 & 9.29 & 24080 & lipid metabolism \\
\hline 97 & Q9FE12 & Peroxiredoxin & P. vulgaris & 12 & 180 & 0.19 & 5.33 & 28776 & redox \\
\hline 98 & Q9SRV5 & 5-methyltetrahydropteroyltriglutamate--homocysteine methyltransferase & A. thaliana & 51 & 506 & 0.19 & 6.51 & 84873 & amino acid metabolism \\
\hline 99 & K3ZW60 & Uncharacterized protein & S. italica & 14 & 316 & 0.18 & 9.76 & 29913 & protein \\
\hline 100 & F2D 448 & Predicted protein & H. vulgare & 16 & 316 & 0.18 & 10.17 & 30607 & protein \\
\hline 101 & P93133 & Isocitrate dehydrogenase [NADP] & E. globulus & 10 & 172 & 0.18 & 6.93 & 47106 & TCA \\
\hline 102 & Q9SXR9 & LeArcA1 protein & S. lycopersicum & 28 & 446 & 0.18 & 7.12 & 36363 & signalling \\
\hline 103 & K3XGJ6 & Uncharacterized protein & S. italica & 13 & 138 & 0.17 & 5.22 & 58129 & protein \\
\hline 104 & O81609 & Nodule-enhanced malate dehydrogenase & P. sativum & 8 & 235 & 0.17 & 7.75 & 42106 & TCA \\
\hline 105 & Q8LG68 & UDP-glucose 6-dehydrogenase & A. thaliana & 21 & 508 & 0.17 & 6.18 & 53591 & stress \\
\hline 106 & Q9LF33 & UDP-glucose 6-dehydrogenase & A. thaliana & 15 & 466 & 0.17 & 6.04 & 53653 & cell wall \\
\hline 107 & H9C956 & 3-deoxy-D-arabino-heptulosonate 7-phosphate synthase & N. benthamiana & 19 & 234 & 0.17 & 8.28 & 53191 & amino acid metabolism \\
\hline 108 & E5GB77 & Adenine nucleotide translocator & C. melo & 5 & 104 & 0.17 & 9.77 & 42664 & transport \\
\hline 109 & A8MRE8 & Triosephosphate isomerase & A. thaliana & 6 & 132 & 0.17 & 7.49 & 32458 & photosynthesis \\
\hline 110 & E5GBS0 & 40S ribosomal protein $\mathrm{S} 24$ & C. melo & 6 & 115 & 0.17 & 10.64 & 15761 & protein \\
\hline 111 & K3XZW6 & Uncharacterized protein & S. italica & 3 & 36 & 0.17 & 10.55 & 15740 & protein \\
\hline 112 & B3TLV4 & 60 S ribosomal protein $\mathrm{L} 27$ & E. guineensis & 4 & 38 & 0.17 & 10.40 & 15833 & protein \\
\hline 113 & B9N744 & Gamma class glutathione transferase EF1Bgamma3 & P. trichocarpa & 18 & 300 & 0.17 & 6.05 & 48353 & protein \\
\hline 114 & K3YZ28 & Uncharacterized protein & S. italica & 5 & 125 & 0.17 & 10.59 & 15971 & protein \\
\hline 115 & A1KXW2 & 1-deoxy-D-xylulose 5-phosphate reductoisomerase & H. brasiliensis & 22 & 154 & 0.16 & 6.00 & 51428 & secondary metabolism \\
\hline 116 & K3XZA2 & Uncharacterized protein & S. italica & 19 & 180 & 0.16 & 9.58 & 23221 & cell \\
\hline 117 & F6H3T7 & Putative uncharacterized protein & V. vinifera & 19 & 192 & 0.16 & 6.11 & 40651 & not assigned \\
\hline 118 & Q8GZR6 & GcpE & S. lycopersicum & 18 & 209 & 0.15 & 6.11 & 82805 & secondary metabolism \\
\hline 119 & E4MXI1 & mRNA, clone: RTFL01-39-B07 & T. halophila & 12 & 172 & 0.15 & 6.98 & 48063 & photosynthesis \\
\hline 120 & E5GCD6 & Multicopper oxidase & C. melo & 29 & 135 & 0.15 & 9.32 & 60810 & not assigned \\
\hline 121 & E5F725 & Putative uncharacterized protein & E. parvulum & 4 & 224 & 0.15 & 7.11 & 42041 & cell wall \\
\hline 122 & B3TLY3 & Ribosomal protein L15 & E. guineensis & 8 & 277 & 0.15 & 11.49 & 24371 & protein \\
\hline 123 & F2D1A7 & Predicted protein & H. vulgare & 35 & 254 & 0.15 & 9.80 & 17886 & protein \\
\hline 124 & K3ZUR5 & Uncharacterized protein & S. italica & 5 & 68 & 0.15 & 6.24 & 36749 & $\begin{array}{l}\text { co-factor and vitamine } \\
\text { metabolism }\end{array}$ \\
\hline 125 & B3F8H6 & Sucrose synthase & N. langsdorffii & 40 & 513 & 0.15 & 6.40 & 92814 & major $\mathrm{CHO}$ metabolism \\
\hline 126 & D6PAY2 & Peptidyl-prolyl cis-trans isomerase & V. hybrid cultivar & 10 & 173 & 0.14 & 8.46 & 18582 & cell \\
\hline 127 & Q4H1G2 & Methionine synthase & B. vulgaris & 49 & 754 & 0.14 & 6.46 & 88546 & amino acid metabolism \\
\hline
\end{tabular}




\begin{tabular}{|c|c|c|c|c|c|c|c|c|c|}
\hline 128 & F2EKH0 & Predicted protein & H. vulgare & 5 & 90 & 0.14 & 4.60 & 31771 & protein \\
\hline 129 & F2CVG4 & Predicted protein & H. vulgare & 27 & 371 & 0.14 & 6.73 & 76549 & cell wall \\
\hline 130 & K3ZA95 & Uncharacterized protein & S. italica & 35 & 69 & 0.14 & 8.53 & 19471 & photosynthesis \\
\hline 131 & Q40556 & Protein phosphatase $2 \mathrm{~A}$ & N. tabacum & 17 & 81 & 0.14 & 5.17 & 66079 & protein \\
\hline 132 & A1YUL9 & Importin subunit alpha & N. benthamiana & 7 & 98 & 0.14 & 5.38 & 59114 & protein \\
\hline 133 & F2DTH4 & Adenylate kinase & H. vulgare & 5 & 111 & 0.13 & 7.81 & 26477 & nucleotide metabolism \\
\hline 134 & F2DGF3 & Predicted protein & H. vulgare & 16 & 372 & 0.13 & 6.81 & 115601 & TCA \\
\hline 135 & B6TJD7 & Isocitrate dehydrogenase subunit 1 & Z. mays & 4 & 55 & 0.13 & 7.14 & 40798 & TCA \\
\hline 136 & Q9ZNX1 & NAD-dependent isocitrate dehydrogenase & N. tabacum & 3 & 154 & 0.13 & 7.52 & 40651 & TCA \\
\hline 137 & A9ZMZ5 & Acetyl-CoA C-acetyltransferase & H. brasiliensis & 12 & 371 & 0.12 & 10.10 & 42039 & secondary metabolism \\
\hline 138 & A8MRH4 & $60 \mathrm{~S}$ ribosomal protein $\mathrm{L} 7-2$ & A. thaliana & 7 & 42 & 0.12 & 6.68 & 21540 & protein \\
\hline 139 & K3YR06 & Uncharacterized protein & S. italica & 16 & 252 & 0.12 & 6.80 & 64225 & protein \\
\hline 140 & K3ZX77 & Uncharacterized protein & S. italica & 10 & 73 & 0.12 & 6.16 & 21045 & protein \\
\hline 141 & D7LW57 & Ketol-acid reductoisomerase & A. lyrata & 9 & 306 & 0.12 & 11.34 & 64346 & amino acid metabolism \\
\hline 142 & K3YR95 & Uncharacterized protein & S. italica & 10 & 172 & 0.12 & 7.65 & 58776 & protein \\
\hline 143 & Q1KUM7 & Putative uncharacterized protein & C. spinosa & 8 & 143 & 0.12 & 6.62 & 59525 & protein \\
\hline 144 & Q9STA4 & Cysteine protease & M. sativa & 2 & 38 & 0.11 & 5.17 & 22875 & protein \\
\hline 145 & Q1EMR1 & Nucleoside-diphopshate-sugar dehydratase & P. major & 9 & 42 & 0.11 & 5.57 & 22710 & cell wall \\
\hline 146 & K3XZE3 & Uncharacterized protein & S. italica & 2 & 105 & 0.11 & 5.27 & 23153 & protein \\
\hline 147 & A8ASG2 & Phosphoenolpyruvate carboxylase & A. arborescens & 21 & 226 & 0.11 & 6.46 & 110813 & glycolysis \\
\hline 148 & B6T0C0 & Heme-binding protein 2 & Z. mays & 2 & 142 & 0.11 & 11.00 & 23860 & tetrapyrrole synthesis \\
\hline 149 & A8MQA1 & $60 \mathrm{~S}$ ribosomal protein $\mathrm{L} 13$ & A. thaliana & 3 & 78 & 0.11 & 5.80 & 23525 & protein \\
\hline 150 & A9PE52 & Gamma class glutathione transferase EF1Bgamma2 & P. trichocarpa & 14 & 124 & 0.11 & 4.78 & 48127 & protein \\
\hline 151 & E5LCX1 & C14 cysteine protease & S. demissum & 6 & 133 & 0.11 & 4.98 & 23974 & protein \\
\hline 152 & E4MY36 & mRNA, clone: RTFL01-37-B16 & T. halophila & 10 & 179 & 0.11 & 6.25 & 63555 & glycolysis \\
\hline 153 & B3TLP3 & Translation elongation factor EF-1 beta chain & E. guineensis & 4 & 83 & 0.11 & 4.69 & 24523 & protein \\
\hline 154 & E3WHD5 & Lipoxygenase & V. hybrid cultivar & 26 & 546 & 0.11 & 8.29 & 100738 & hormone metabolism \\
\hline 155 & C8YNG6 & 3-ketoacyl CoA thiolase 1 & P. hybrida & 13 & 97 & 0.11 & 6.55 & 49405 & amino acid metabolism \\
\hline 156 & B6TPE4 & Glutamate-1-semialdehyde 2,1-aminomutase & Z. mays & 6 & 75 & 0.10 & 6.55 & 50223 & tetrapyrrole synthesis \\
\hline 157 & Q6R9J5 & ATPase subunit 4 & Z. mays & 4 & 39 & 0.10 & 8.48 & 24941 & mitoETC \\
\hline 158 & Q2LFC3 & AGO1-2 & N. benthamiana & 19 & 261 & 0.10 & 9.31 & 109989 & development \\
\hline 159 & Q9LLR1 & Acetyl-CoA carboxylase & G. $\max$ & 10 & 223 & 0.10 & 7.50 & 59306 & lipid metabolism \\
\hline
\end{tabular}




\begin{tabular}{|c|c|c|c|c|c|c|c|c|c|}
\hline 160 & K4A6V8 & Uncharacterized protein & S. italica & 6 & 151 & 0.10 & 7.03 & 70549 & glycolysis \\
\hline 161 & E5GBL8 & ATP-dependent clp protease & C. melo & 19 & 256 & 0.10 & 7.23 & 103416 & protein \\
\hline 162 & B9H5E3 & Predicted protein & P. trichocarpa & 15 & 98 & 0.10 & 9.01 & 61685 & not assigned \\
\hline 163 & Q9FZG9 & Putative pectinesterase & A. thaliana & 19 & 111 & 0.10 & 9.45 & 60580 & cell wall \\
\hline 164 & K3ZD99 & Uncharacterized protein & S. italica & 10 & 64 & 0.10 & 4.68 & 26325 & transport \\
\hline 165 & F2DLC2 & Proteasome subunit alpha type & H. vulgare & 4 & 177 & 0.10 & 8.63 & 26087 & protein \\
\hline 166 & F2DP49 & Predicted protein & H. vulgare & 8 & 104 & 0.10 & 9.16 & 25913 & hormone metabolism \\
\hline 167 & K3ZSX0 & Uncharacterized protein & S. italica & 6 & 72 & 0.10 & 7.64 & 53564 & amino acid metabolism \\
\hline 168 & Q9XEN8 & Annexin & N. tabacum & 6 & 43 & 0.10 & 5.53 & 36029 & cell \\
\hline 169 & D3GQL1 & Aconitate hydratase 3 & C. clementina & 36 & 529 & 0.10 & 6.30 & 98669 & TCA \\
\hline 170 & Q2LFC4 & AGO1-1 & $N$. benthamiana & 19 & 154 & 0.10 & 9.45 & 118109 & development \\
\hline 171 & Q1HG95 & Sucrose synthase & V. album & 26 & 100 & 0.10 & 6.09 & 92607 & major $\mathrm{CHO}$ metabolism \\
\hline 172 & F2D8B8 & Proteasome subunit alpha type & H. vulgare & 3 & 111 & 0.09 & 7.43 & 27434 & protein \\
\hline 173 & F2EKQ2 & Predicted protein & H. vulgare & 9 & 58 & 0.09 & 10.33 & 28399 & protein \\
\hline 174 & Q5GAQ3 & Putative alanine aminotransferase & Z. mays & 6 & 110 & 0.09 & 5.64 & 57003 & amino acid metabolism \\
\hline 175 & F2Z9R1 & Glucose-6-phosphate 1-dehydrogenase & N. benthamiana & 13 & 123 & 0.09 & 6.49 & 58894 & OPP \\
\hline 176 & G5DW29 & Putative calreticulin & S. latifolia & 6 & 54 & 0.09 & 6.23 & 48820 & signalling \\
\hline 177 & K3XVH0 & Uncharacterized protein & S. italica & 13 & 193 & 0.09 & 6.62 & 80203 & photosynthesis \\
\hline 178 & Q8H945 & Phosphoenolpyruvate carboxylase & L. japonicus & 15 & 206 & 0.09 & 5.88 & 111020 & glycolysis \\
\hline 179 & B6U0V6 & Endoplasmin & Z. mays & 18 & 409 & 0.09 & 4.97 & 92831 & stress \\
\hline 180 & B7SA66 & Hsp90-like protein & D. glomerata & 21 & 373 & 0.09 & 4.97 & 92918 & stress \\
\hline 181 & F2E2M4 & Predicted protein & H. vulgare & 12 & 144 & 0.09 & 8.51 & 92002 & stress \\
\hline 182 & Q8H6B6 & Chaperone protein $\mathrm{HtpG}$ & $X . v i s c o s a$ & 25 & 344 & 0.09 & 5.29 & 92957 & stress \\
\hline 183 & K017G7 & Thioredoxin peroxidase & N. tabacum & 8 & 64 & 0.09 & 8.03 & 29972 & redox \\
\hline 184 & F2CT97 & Predicted protein & H. vulgare & 2 & 43 & 0.09 & 5.02 & 30094 & not assigned \\
\hline 185 & Q9M2T8 & 2-oxoglutarate dehydrogenase, E1 subunit-like protein & A. thaliana & 12 & 139 & 0.08 & 7.14 & 114888 & TCA \\
\hline 186 & B6TLT1 & Serine/threonine-protein phosphatase $2 \mathrm{~A} 65 \mathrm{kDa}$ regulatory subunit A beta isoform & Z. mays & 20 & 79 & 0.08 & 5.03 & 66318 & protein \\
\hline 187 & F6HGZ1 & Pectinesterase & V. vinifera & 9 & 143 & 0.08 & 7.49 & 63329 & cell wall \\
\hline 188 & Q39834 & Clathrin heavy chain & G. $\max$ & 22 & 249 & 0.08 & 5.55 & 194430 & cell \\
\hline 189 & E4MWY0 & mRNA, clone: RTFL01-19-L03 & T. halophila & 19 & 102 & 0.08 & 9.39 & 120492 & development \\
\hline 190 & Q2LFC1 & AGO4-2 & N. benthamiana & 13 & 136 & 0.08 & 6.37 & 102331 & RNA \\
\hline 191 & I0J3D8 & Proteasome subunit beta type & A. halleri & 3 & 54 & 0.08 & 6.06 & 33367 & protein \\
\hline
\end{tabular}




\begin{tabular}{|c|c|c|c|c|c|c|c|c|c|}
\hline 192 & I6LE48 & Uncharacterized protein & D. excelsa & 7 & 62 & 0.08 & 5.03 & 33659 & development \\
\hline 193 & A0FKE6 & Chloroplast threonine deaminase 1 & S. lycopersicum & 5 & 135 & 0.08 & 8.78 & 66653 & amino acid metabolism \\
\hline 194 & А9P9A2 & Putative uncharacterized protein & P. trichocarpa & 23 & 268 & 0.08 & 9.36 & 46272 & RNA \\
\hline 195 & Q8LDR5 & Putative GTP-binding protein & A. thaliana & 9 & 46 & 0.08 & 6.81 & 44776 & OPP \\
\hline 196 & K3Y4M8 & Uncharacterized protein & S. italica & 18 & 256 & 0.07 & 5.39 & 194893 & cell \\
\hline 197 & F2E797 & Predicted protein & H. vulgare & 12 & 79 & 0.07 & 5.71 & 47111 & TCA \\
\hline 198 & $\mathrm{C} 5 \mathrm{X} 277$ & Putative uncharacterized protein $\mathrm{Sb} 02 \mathrm{~g} 026300$ & S. bicolor & 3 & 97 & 0.07 & 5.72 & 47330 & redox \\
\hline 199 & F2CTR2 & Predicted protein & H. vulgare & 8 & 81 & 0.07 & 6.99 & 59167 & protein \\
\hline 200 & A8MRF3 & $60 \mathrm{~S}$ ribosomal protein $\mathrm{L} 18 \mathrm{a}-1$ & A. thaliana & 3 & 77 & 0.07 & 9.73 & 36337 & misc \\
\hline 201 & В6ТВ29 & Fructokinase-2 & Z. mays & 10 & 87 & 0.07 & 5.58 & 35875 & major $\mathrm{CHO}$ metabolism \\
\hline 202 & Q9SF16 & AT3g11830/F26K24_12 & A. thaliana & 9 & 92 & 0.07 & 6.39 & 60195 & protein \\
\hline 203 & Q7XJH9 & Transaldolase & S. lycopersicum & 9 & 55 & 0.07 & 5.96 & 48062 & OPP \\
\hline 204 & K4A9K9 & Uncharacterized protein & S. italica & 3 & 63 & 0.07 & 5.83 & 52168 & secondary metabolism \\
\hline 205 & Q19TV8 & UDP-glucose pyrophosphorylase & C. melo & 11 & 61 & 0.07 & 7.17 & 52201 & glycolysis \\
\hline 206 & $\mathrm{~K} 4 \mathrm{ACC} 4$ & Uncharacterized protein & S. italica & 8 & 64 & 0.06 & 8.18 & 38305 & protein \\
\hline 207 & Q39986 & Cysteine proteinase & Hemerocallis sp. & 2 & 90 & 0.06 & 5.66 & 40006 & protein \\
\hline 208 & O78327 & Transketolase 1 & C. аппиит & 10 & 103 & 0.06 & 6.62 & 80398 & OPP \\
\hline 209 & B9RI89 & Serine-threonine protein kinase, plant-type, putative & R. communis & 11 & 144 & 0.06 & 5.50 & 40375 & not assigned \\
\hline 210 & K4A8Y9 & Uncharacterized protein & S. italica & 3 & 157 & 0.06 & 7.61 & 53531 & TCA \\
\hline 211 & E4MXV1 & mRNA, clone: RTFL01-35-G02 & T. halophila & 2 & 50 & 0.06 & 6.00 & 40626 & protein \\
\hline 212 & K4A6E4 & Uncharacterized protein & S. italica & 8 & 64 & 0.06 & 5.16 & 83215 & protein \\
\hline 213 & E4MWY6 & Glucose-6-phosphate isomerase & T. halophila & 4 & 69 & 0.06 & 5.60 & 67312 & glycolysis \\
\hline 214 & В9HJ23 & Predicted protein & P. trichocarpa & 13 & 199 & 0.06 & 6.54 & 101700 & misc \\
\hline 215 & B4FYM0 & Acetyl-CoA acetyltransferase, cytosolic 2 & Z. mays & 2 & 99 & 0.06 & 6.77 & 43450 & secondary metabolism \\
\hline 216 & B0M183 & Acetylcholinesterase & M. atropurpureum & 4 & 117 & 0.06 & 7.66 & 43320 & misc \\
\hline 217 & E4MXR5 & mRNA, clone: RTFL01-40-M04 & T. halophila & 15 & 153 & 0.06 & 5.90 & 73489 & stress \\
\hline 218 & Q2I826 & Monodehydroascorbate reductase III & P. patens & 4 & 29 & 0.06 & 5.92 & 46597 & redox \\
\hline 219 & O65879 & Translation initiation factor & P. sativum & 7 & 75 & 0.06 & 7.46 & 46184 & protein \\
\hline 220 & E5GBW6 & $26 \mathrm{~S}$ proteasome non-ATPase regulatory subunit & C. melo & 2 & 76 & 0.06 & 5.82 & 44456 & glycolysis \\
\hline 221 & B6TVN4 & Phospholipase C & Z. mays & 7 & 129 & 0.05 & 8.43 & 46868 & signalling \\
\hline 222 & K3XFX7 & Uncharacterized protein & S. italica & 2 & 96 & 0.05 & 6.68 & 64026 & glycolysis \\
\hline 223 & Q8LGJ6 & Phosphoglycerate dehydrogenase-like protein & A. thaliana & 3 & 103 & 0.05 & 6.76 & 63556 & amino acid metabolism \\
\hline
\end{tabular}




\begin{tabular}{|c|c|c|c|c|c|c|c|c|c|}
\hline 224 & Q93YG1 & Monodehydroascorbate reductase & M. crystallinum & 6 & 107 & 0.05 & 7.65 & 51944 & redox \\
\hline 225 & C5WQZ5 & Putative uncharacterized protein Sb01g041210 & S. bicolor & 2 & 110 & 0.05 & 5.66 & 51802 & protein \\
\hline 226 & F2E941 & Predicted protein & H. vulgare & 8 & 140 & 0.05 & 6.67 & 52201 & protein \\
\hline 227 & G3CHK8 & Eukaryotic translation initiation factor 3 subunit $\mathrm{E}$ & P. trifoliata & 5 & 41 & 0.05 & 6.14 & 51916 & protein \\
\hline 228 & F2DDG9 & Predicted protein & H. vulgare & 3 & 81 & 0.05 & 7.33 & 52441 & cell \\
\hline 229 & Q43057 & Aspartate aminotransferase & P. miliaceum & 4 & 47 & 0.05 & 6.81 & 48154 & amino acid metabolism \\
\hline 230 & F2CTK4 & Predicted protein & H. vulgare & 16 & 65 & 0.05 & 9.04 & 83843 & lipid metabolism \\
\hline 231 & F2DIS4 & Malic enzyme & H. vulgare & 2 & 48 & 0.05 & 7.40 & 68411 & TCA \\
\hline 232 & $\mathrm{O} 24135$ & Citrate synthase & N. tabacum & 5 & 100 & 0.05 & 7.85 & 52744 & TCA \\
\hline 233 & Q9LLA9 & Fatty acid hydroperoxide lyase & S. lycopersicum & 3 & 124 & 0.05 & 6.81 & 53737 & misc \\
\hline 234 & Q9S768 & Alanine aminotransferase & O. sativa & 3 & 180 & 0.05 & 6.65 & 53130 & amino acid metabolism \\
\hline 235 & K3YRV8 & Uncharacterized protein & S. italica & 5 & 28 & 0.04 & 6.84 & 55802 & TCA \\
\hline 236 & Q38766 & Victorin binding protein & A. sativa & 9 & 114 & 0.04 & 8.21 & 112298 & photosynthesis \\
\hline 237 & G2XK63 & Putative uncharacterized protein & Z. mays & 3 & 68 & 0.04 & 5.82 & 57701 & protein \\
\hline 238 & Q9SZ27 & Putative aspartate-tRNA ligase & A. thaliana & 2 & 41 & 0.04 & 6.60 & 57768 & protein \\
\hline 239 & E4MX61 & mRNA, clone: RTFL01-20-P01 & T. halophila & 10 & 72 & 0.04 & 7.27 & 53948 & glycolysis \\
\hline 240 & K3XWI6 & Uncharacterized protein & S. italica & 10 & 166 & 0.04 & 6.96 & 55489 & glycolysis \\
\hline 241 & F2D3S8 & Serine hydroxymethyltransferase & H. vulgare & 7 & 44 & 0.04 & 6.37 & 56215 & C1-metabolism \\
\hline 242 & B5BLW2 & Carotenoid cleavage dioxygenase 1 & M. truncatula & 5 & 39 & 0.04 & 5.83 & 61063 & secondary metabolism \\
\hline 243 & A8J1X8 & Aspartyl-tRNA synthetase & C. reinhardtii & 3 & 45 & 0.04 & 6.35 & 60686 & protein \\
\hline 244 & E1Z4U0 & Putative uncharacterized protein & C. variabilis & 15 & 165 & 0.04 & 6.79 & 60152 & protein \\
\hline 245 & F2DUH3 & Predicted protein & H. vulgare & 5 & 47 & 0.04 & 6.76 & 60964 & protein \\
\hline 246 & B4FQM2 & Pyrophosphate--fructose 6-phosphate 1-phosphotransferase beta subunit & Z. mays & 9 & 80 & 0.04 & 6.95 & 61607 & glycolysis \\
\hline 247 & B6T715 & Aldehyde dehydrogenase & Z. mays & 2 & 97 & 0.04 & 5.63 & 59108 & fermentation \\
\hline 248 & F2E6F3 & Coatomer subunit gamma & H. vulgare & 2 & 159 & 0.04 & 5.17 & 100353 & cell \\
\hline 249 & K3XVB0 & Uncharacterized protein & S. italica & 6 & 125 & 0.04 & 7.81 & 87653 & protein \\
\hline 250 & Q8L5C2 & $110 \mathrm{kDa} 4 \mathrm{SNc}-T u d o r$ domain protein & P. sativum & 4 & 95 & 0.04 & 7.36 & 108600 & RNA \\
\hline 251 & K3XE87 & Uncharacterized protein & S. italica & 6 & 70 & 0.04 & 5.90 & 106127 & cell \\
\hline 252 & E5GBL0 & Short-chain dehydrogenase/reductase & C. melo & 9 & 78 & 0.04 & 6.89 & 108844 & RNA \\
\hline 253 & Q9LLB8 & Exoglucanase & Z. mays & 9 & 138 & 0.04 & 7.34 & 67356 & cell wall \\
\hline 254 & K4A4Z8 & Uncharacterized protein & S. italica & 7 & 40 & 0.03 & 6.96 & 137642 & cell \\
\hline 255 & B6SVF4 & Oligopeptide transporter 9 & Z. mays & 6 & 44 & 0.03 & 6.47 & 87421 & transport \\
\hline
\end{tabular}




\begin{tabular}{|c|c|c|c|c|c|c|c|c|c|}
\hline 256 & K3YKV0 & Uncharacterized protein & S. italica & 16 & 127 & 0.03 & 5.54 & 87462 & cell \\
\hline 257 & A6MIZ2 & Trehalose-phosphate synthase 5 & P. patens & 5 & 104 & 0.03 & 8.09 & 97638 & signalling \\
\hline 258 & Q9ZVY6 & $\mathrm{T} 25 \mathrm{~N} 20.17$ & A. thaliana & 2 & 46 & 0.03 & 5.58 & 90732 & protein \\
\hline 259 & A2JGX1 & Beta-galactosidase & S. lycopersicum & 3 & 55 & 0.03 & 5.29 & 98181 & misc \\
\hline 260 & B2KNE6 & Phospholipase D & H. annииs & 7 & 95 & 0.03 & 5.52 & 92315 & lipid metabolism \\
\hline 261 & E4MVK9 & mRNA, clone: RTFL01-02-O16 & T. halophila & 3 & 128 & 0.03 & 8.41 & 92372 & lipid metabolism \\
\hline 262 & Q9XFX7 & Phospholipase D & C. plantagineum & 5 & 37 & 0.03 & 5.66 & 92228 & lipid metabolism \\
\hline 263 & A8IM71 & Coatomer subunit gamma & C. reinhardtii & 4 & 38 & 0.03 & 5.58 & 97872 & cell \\
\hline 264 & F6HZ11 & Lipoxygenase & V. vinifera & 5 & 74 & 0.02 & 6.04 & 99463 & hormone metabolism \\
\hline 265 & J3MFV3 & Lipoxygenase & O. brachyantha & 8 & 43 & 0.02 & 6.77 & 103742 & hormone metabolism \\
\hline 266 & Q4LDR0 & Heat shock protein & S. lycopersicum & 5 & 81 & 0.02 & 6.55 & 110564 & stress \\
\hline 267 & Q42793 & Acetyl CoA carboxylase & G. $\max$ & 5 & 46 & 0.01 & 6.32 & 253225 & lipid metabolism \\
\hline 268 & E4MXV2 & mRNA, clone: RTFL01-41-F15 & T. halophila & 11 & 60 & - & 10.49 & 64768 & secondary metabolism \\
\hline 269 & В3ТМ07 & Ribosomal protein L17 & E. guineensis & 13 & 88 & - & 6.09 & 20744 & protein \\
\hline
\end{tabular}

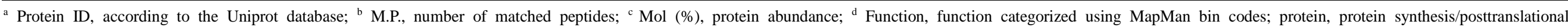

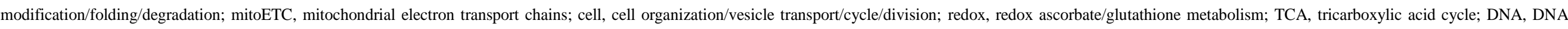
synthesis/repair; OPP, oxidative pentose phosphate; RNA, RNA processing/regulation of transcription; and misc, miscellaneous. 
Supplemental Table 4. Proteins Identified in L. japonica Immature Flower Buds at Fraction of Flow Through of pH 7 using Combinatorial Peptide Ligand Libraries Fractionation.

\begin{tabular}{|c|c|c|c|c|c|c|c|c|c|}
\hline No. & Protein ID $^{\text {a }}$ & Description & Species & M.P. ${ }^{\mathrm{b}}$ & Score & $\operatorname{Mol}(\%)^{\mathrm{c}}$ & $\mathrm{pI}$ & Mass (Da) & Function $^{\mathrm{d}}$ \\
\hline 1 & Q9M6B3 & Malate dehydrogenase & V. vinifera & 141 & 3168 & 1.53 & 8.62 & 37137 & TCA \\
\hline 2 & C4B8E5 & Glyceraldehyde-3-phosphate dehydrogenase & T. gesneriana & 101 & 2728 & 1.50 & 7.96 & 20964 & glycolysis \\
\hline 3 & Q9GD57 & Ribulose 5-bisphosphate carboxylase, large subunit & P. yemenensis & 183 & 1688 & 1.44 & 6.89 & 51857 & photosynthesis \\
\hline 4 & Q6PKU2 & Putative glyceraldehyde-3-phosphate dehydrogenase & O. minor & 84 & 2093 & 1.24 & 7.09 & 22122 & glycolysis \\
\hline 5 & H1ZY49 & Translation elongation factor 1-alpha & L. maackii & 15 & 104 & 1.21 & 5.00 & 11619 & protein \\
\hline 6 & C6GFP3 & ATP synthase subunit B. & G. hirsutum & 120 & 2270 & 1.12 & 6.29 & 59987 & mitoETC \\
\hline 8 & F2D884 & Predicted protein & H. vulgare & 90 & 1448 & 0.90 & 5.19 & 71476 & stress \\
\hline 9 & Q645N1 & Malate dehydrogenase & S. lycopersicum & 60 & 1475 & 0.90 & 8.72 & 36357 & TCA \\
\hline 10 & Q5KTY2 & Ribulose-1,5-bisphosphate carboxylase large subunit & A. sutepensis & 119 & 1409 & 0.89 & 6.92 & 51143 & photosynthesis \\
\hline 11 & Q8MCX1 & Ribulose-1,5-bisphosphate carboxylase large subunit & C. axillaris & 120 & 1282 & 0.86 & 6.58 & 52386 & photosynthesis \\
\hline 12 & K4ACE3 & Uncharacterized protein & S. italica & 116 & 1946 & 0.80 & 6.09 & 35803 & TCA \\
\hline 13 & K3XJN7 & Uncharacterized protein & S. italica & 49 & 938 & 0.75 & 7.85 & 35629 & TCA \\
\hline 14 & Q9TKH9 & Ribulose 1,5-bisphosphate carboxylase large subunit & H. albomarginata & 108 & 1548 & 0.75 & 6.37 & 51833 & photosynthesis \\
\hline 16 & Q9TKG9 & Ribulose 1,5-bisphosphate carboxylase large subunit & R. tomentosum & 136 & 1336 & 0.69 & 7.11 & 52772 & photosynthesis \\
\hline 17 & J3RTS9 & Glyceraldehyde-3-phosphate dehydrogenase & C. pentagona & 123 & 2686 & 0.68 & 6.72 & 32382 & glycolysis \\
\hline 18 & Q9FEM4 & Catalase & B. pendula & 25 & 219 & 0.66 & 5.48 & 17247 & redox \\
\hline 19 & Q7DM38 & Ribulose bisphosphate carboxylase small chain & P. vulgaris & 34 & 172 & 0.64 & 8.43 & 15877 & photosynthesis \\
\hline 20 & F2D6I8 & Predicted protein & H. vulgare & 67 & 1731 & 0.62 & 7.14 & 36874 & glycolysis \\
\hline 21 & B3TLL4 & Triosephosphate isomerase & E. guineensis & 22 & 160 & 0.59 & 6.23 & 27476 & glycolysis \\
\hline 22 & Q08II7 & Heat shock protein 70 -like protein & L. sativa & 10 & 366 & 0.58 & 10.33 & 12539 & stress \\
\hline 23 & E5GBV9 & Glyceraldehyde-3-phosphate dehydrogenase & C. melo & 103 & 1310 & 0.58 & 6.62 & 36471 & glycolysis \\
\hline 24 & Q5EBY7 & Heat shock protein 70 & Z. mays & 46 & 680 & 0.56 & 4.78 & 41240 & stress \\
\hline 25 & Q5ZFR7 & Malate dehydrogenase & P. major & 79 & 1670 & 0.53 & 6.54 & 36026 & TCA \\
\hline 26 & D7NHW9 & 2-phospho-D-glycerate hydrolase & P. trifoliata & 61 & 1595 & 0.53 & 5.78 & 47986 & glycolysis \\
\hline 27 & $\mathrm{~F} 2 \mathrm{E} 4 \mathrm{C} 2$ & Predicted protein & H. vulgare & 64 & 1096 & 0.47 & 5.25 & 72202 & stress \\
\hline 28 & O81830 & Putative uncharacterized protein AT4g27270 & A. thaliana & 11 & 123 & 0.47 & 6.79 & 22355 & lipid metabolism \\
\hline 31 & E0D6S1 & Glyceraldehyde 3-phosphate dehydrogenase & G. bicolor & 61 & 1472 & 0.43 & 6.57 & 36957 & glycolysis \\
\hline
\end{tabular}




\begin{tabular}{|c|c|c|c|c|c|c|c|c|c|}
\hline 32 & B6SHD3 & Malate dehydrogenase & Z. mays & 76 & 788 & 0.43 & 8.32 & 42623 & TCA \\
\hline 33 & G0WP59 & Superoxide dismutase $[\mathrm{Cu}-\mathrm{Zn}]$ & W. somnifera & 17 & 332 & 0.43 & 5.64 & 15782 & redox \\
\hline 34 & K3Z913 & Uncharacterized protein & S. italica & 28 & 543 & 0.43 & 9.47 & 26793 & cell \\
\hline 35 & Q40463 & NTGB2 & N. tabacum & 7 & 140 & 0.42 & 6.19 & 15827 & signalling \\
\hline 36 & A4ZSZ4 & Actin & P. trichocarpa & 22 & 330 & 0.41 & 5.49 & 41913 & cell \\
\hline 37 & Q2TFP1 & Tubulin B4 & G. $\max$ & 35 & 465 & 0.40 & 4.83 & 51083 & cell \\
\hline 38 & E5GBV8 & Glyceraldehyde-3-phosphate dehydrogenase & C. melo & 66 & 1258 & 0.40 & 7.12 & 36754 & glycolysis \\
\hline 39 & K3ZUR5 & Uncharacterized protein & S. italica & 24 & 676 & 0.39 & 6.24 & 36749 & co-factor and vitamine metabolism \\
\hline 40 & Q06Z74 & Fibrillin 4 & C. canephora & 14 & 343 & 0.38 & 8.88 & 32064 & cell \\
\hline 41 & F2CTZ5 & Predicted protein & H. vulgare & 29 & 803 & 0.37 & 5.38 & 68752 & transport \\
\hline 42 & B3TLQ5 & $60 \mathrm{~S}$ ribosomal protein $\mathrm{L} 11$ & E. guineensis & 14 & 203 & 0.37 & 9.92 & 20950 & protein \\
\hline 43 & B6T1W9 & $60 \mathrm{~S}$ ribosomal protein $\mathrm{L} 12$ & Z. mays & 9 & 152 & 0.37 & 9.85 & 17770 & protein \\
\hline 44 & F2DAC4 & Predicted protein & H. vulgare & 3 & 73 & 0.36 & 7.18 & 12136 & not assigned \\
\hline 45 & E4MXI2 & mRNA, clone: RTFL01-39-D20 & T. halophila & 41 & 682 & 0.36 & 5.21 & 73890 & stress \\
\hline 46 & Q6T379 & Triosephosphate isomerase & S. chacoense & 17 & 334 & 0.36 & 5.99 & 27251 & glycolysis \\
\hline 47 & D0ELH5 & Peptidyl-prolyl cis-trans isomerase & C. sinensis & 12 & 274 & 0.35 & 8.46 & 18310 & cell \\
\hline 48 & D6PAY2 & Peptidyl-prolyl cis-trans isomerase & V. hybrid cultivar & 14 & 240 & 0.34 & 8.46 & 18582 & cell \\
\hline 49 & Q9SW73 & Isocitrate dehydrogenase [NADP] & C. limon & 14 & 237 & 0.34 & 6.95 & 46823 & TCA \\
\hline 50 & O81609 & Nodule-enhanced malate dehydrogenase & P. sativum & 17 & 520 & 0.33 & 7.75 & 42106 & TCA \\
\hline 51 & B2VQE0 & Methionine synthase & O. ramosa & 106 & 1285 & 0.33 & 6.40 & 84862 & amino acid metabolism \\
\hline 52 & A8MQP1 & Adenosylhomocysteinase 1 & A. thaliana & 27 & 248 & 0.33 & 5.16 & 36022 & amino acid metabolism \\
\hline 53 & O22402 & GDP dissociation inhibitor & N. tabacum & 21 & 104 & 0.32 & 5.67 & 50127 & signalling \\
\hline 54 & B6SJZ8 & Glyceraldehyde-3-phosphate dehydrogenase, cytosolic & Z. mays & 66 & 1231 & 0.32 & 6.96 & 36614 & glycolysis \\
\hline 55 & Q5IA96 & Cytochrome oxidase subunit II & A. trichopoda & 2 & 91 & 0.32 & 4.75 & 9794 & mitoETC \\
\hline 56 & G5DVX2 & Phosphoglycerate kinase & S. latifolia & 48 & 1078 & 0.31 & 7.05 & 51352 & photosynthesis \\
\hline 57 & B6T9G3 & Alpha-1,4-glucan-protein synthase 1 & Z. mays & 34 & 411 & 0.31 & 6.58 & 41278 & cell wall \\
\hline 58 & Q19TV8 & UDP-glucose pyrophosphorylase & C. melo & 33 & 300 & 0.31 & 7.17 & 52201 & glycolysis \\
\hline 59 & K3XL57 & Uncharacterized protein & S. italica & 17 & 155 & 0.31 & 5.48 & 27435 & glycolysis \\
\hline 60 & K3XFR6 & Uncharacterized protein & S. italica & 40 & 569 & 0.30 & 7.46 & 66448 & TCA \\
\hline 61 & Q8MCY2 & Ribulose bisphosphate carboxylase large chain & V. baccifera & 44 & 444 & 0.30 & 6.64 & 48739 & photosynthesis \\
\hline 62 & A4UMS1 & Myo-inositol-1-phosphate synthase isoform 1 & $X$. viscosa & 37 & 655 & 0.29 & 5.41 & 56899 & minor CHO metabolism \\
\hline 63 & I3NVX2 & V-type ATPase subunit A & S. europaea & 38 & 506 & 0.29 & 5.20 & 68845 & transport \\
\hline
\end{tabular}




\begin{tabular}{|c|c|c|c|c|c|c|c|c|c|}
\hline 64 & B6EBD6 & Heat shock protein $90-2$ & G. $\max$ & 43 & 448 & 0.29 & 5.07 & 80392 & stress \\
\hline 65 & F2DAU4 & Predicted protein & H. vulgare & 24 & 404 & 0.28 & 7.56 & 62696 & protein \\
\hline 66 & Q7XJH9 & Transaldolase & S. lycopersicum & 15 & 286 & 0.28 & 5.96 & 48062 & OPP \\
\hline 67 & $\mathrm{O} 23254$ & Serine hydroxymethyltransferase & A. thaliana & 50 & 647 & 0.28 & 7.23 & 52141 & C1-metabolism \\
\hline 68 & H9NIE1 & Cell division cycle protein 48 & C. sinensis & 37 & 811 & 0.28 & 5.29 & 90644 & cell \\
\hline 69 & Q8GZD5 & Xyloglucan endotransglycosylase & P. tremula & 31 & 368 & 0.27 & 7.75 & 34481 & cell wall \\
\hline 70 & Q6ZXH7 & Putative ascorbate peroxidase & P. canadensis & 12 & 153 & 0.27 & 4.97 & 22506 & redox \\
\hline 71 & A9ZMZ5 & Acetyl-CoA C-acetyltransferase & H. brasiliensis & 34 & 1072 & 0.27 & 6.68 & 42039 & secondary metabolism \\
\hline 72 & B6TPE4 & Glutamate-1-semialdehyde 2,1-aminomutase & Z. mays & 15 & 158 & 0.27 & 6.55 & 50223 & tetrapyrrole synthesis \\
\hline 73 & Q9AXR6 & ATP:citrate lyase & C. аппиит & 33 & 551 & 0.26 & 7.39 & 66376 & TCA \\
\hline 74 & Q8LC80 & Putative calcium-binding protein, calreticulin & A. thaliana & 37 & 660 & 0.25 & 4.53 & 48385 & signalling \\
\hline 75 & A1BQW9 & Transketolase & N. attenuata & 23 & 58 & 0.25 & 6.80 & 32653 & OPP \\
\hline 76 & B6TWN7 & Elongation factor 1-alpha & Z. mays & 17 & 103 & 0.25 & 9.07 & 49594 & protein \\
\hline 77 & O48903 & Malate dehydrogenase & M. sativa & 28 & 607 & 0.24 & 8.29 & 38397 & gluconeogenesis \\
\hline 78 & F2DBW7 & Predicted protein & H. vulgare & 5 & 31 & 0.24 & 5.10 & 12439 & protein \\
\hline 79 & G9MA91 & Fructose-bisphosphate aldolase & L. grandiflorum & 9 & 295 & 0.24 & 9.55 & 37690 & glycolysis \\
\hline 80 & A2Q5W0 & Alpha-tubulin & M. truncatula & 15 & 349 & 0.24 & 6.28 & 50457 & cell \\
\hline 81 & Q5ZF83 & Putative uncharacterized protein & P. major & 23 & 116 & 0.24 & 4.37 & 25390 & cell wall \\
\hline 82 & F2D690 & Predicted protein & H. vulgare & 4 & 139 & 0.24 & 7.28 & 12678 & protein \\
\hline 83 & Q9ATF4 & Ribosomal protein L33 & C. sativa & 3 & 172 & 0.23 & 10.58 & 12831 & protein \\
\hline 84 & D0EJY9 & Molecular chaperone Hsp90-3 & N. benthamiana & 33 & 439 & 0.22 & 5.02 & 80638 & stress \\
\hline 85 & Q84RD8 & Adenosylhomocysteinase & M. truncatula & 37 & 407 & 0.22 & 6.01 & 53790 & amino acid metabolism \\
\hline 86 & B6TGL7 & Enolase & Z. mays & 10 & 321 & 0.22 & 6.23 & 50512 & glycolysis \\
\hline 87 & B6TN41 & Regulator of ribonuclease activity A & Z. mays & 4 & 63 & 0.22 & 6.18 & 18390 & C1-metabolism \\
\hline 88 & K3XZA2 & Uncharacterized protein & S. italica & 18 & 232 & 0.21 & 9.58 & 23221 & cell \\
\hline 89 & Q645M9 & Glyoxisomal malate dehydrogenase & S. lycopersicum & 17 & 323 & 0.21 & 7.94 & 38007 & gluconeogenesis \\
\hline 90 & K3XXC5 & Uncharacterized protein & S. italica & 55 & 773 & 0.21 & 7.06 & 42409 & glycolysis \\
\hline 91 & Q9SXX5 & Fructose-bisphosphate aldolase & N. paniculata & 9 & 307 & 0.20 & 6.79 & 43074 & photosynthesis \\
\hline 92 & Q4H1G1 & Adenosylhomocysteinase & B. vulgaris & 32 & 204 & 0.20 & 6.19 & 54052 & amino acid metabolism \\
\hline 93 & A9CM22 & Voltage-dependent anion channel & N. tabacum & 4 & 142 & 0.20 & 8.32 & 29694 & transport \\
\hline 94 & K3XVH0 & Uncharacterized protein & S. italica & 52 & 176 & 0.20 & 6.62 & 80203 & photosynthesis \\
\hline 95 & Q7XAE2 & Putative fructokinase 2 & $P$. integrifolia & 39 & 319 & 0.20 & 5.35 & 35181 & major CHO metabolism \\
\hline
\end{tabular}




\begin{tabular}{|c|c|c|c|c|c|c|c|c|c|}
\hline 96 & Q9SP13 & Nucleoside diphosphate kinase & P. sativum & 8 & 66 & 0.19 & 9.32 & 25362 & not assigned \\
\hline 97 & B4FPK8 & Cytochrome b5 & Z. mays & 4 & 85 & 0.19 & 5.30 & 14958 & redox \\
\hline 98 & Q8GZD8 & Leucine aminopeptidase 2, chloroplastic & S. lycopersicum & 10 & 134 & 0.19 & 7.80 & 60813 & protein \\
\hline 99 & F2CQ27 & Predicted protein & H. vulgare & 8 & 67 & 0.19 & 6.66 & 25460 & signalling \\
\hline 100 & K3YVE3 & Uncharacterized protein & S. italica & 20 & 204 & 0.19 & 5.53 & 25864 & protein \\
\hline 101 & Q6VWJ5 & Fructokinase 3 & S. lycopersicum & 15 & 124 & 0.19 & 5.80 & 41803 & major $\mathrm{CHO}$ metabolism \\
\hline 102 & F2D448 & Predicted protein & H. vulgare & 16 & 125 & 0.19 & 4.84 & 30607 & protein \\
\hline 103 & A8MQR4 & $60 \mathrm{~S}$ acidic ribosomal protein $\mathrm{P} 0-2$ & A. thaliana & 11 & 160 & 0.19 & 10.17 & 30656 & protein \\
\hline 104 & D3GQL1 & Aconitate hydratase 3 & C. clementina & 44 & 820 & 0.19 & 6.30 & 98669 & TCA \\
\hline 105 & Q3KN68 & Isoflavone reductase-like protein 5 & V. vinifera & 17 & 170 & 0.19 & 6.10 & 33865 & secondary metabolism \\
\hline 106 & G5DXP1 & Fructose-bisphosphate aldolase & S. latifolia & 7 & 285 & 0.18 & 6.16 & 42771 & photosynthesis \\
\hline 107 & E5GBS0 & 40 S ribosomal protein $\mathrm{S} 24$ & C. melo & 3 & 85 & 0.18 & 10.64 & 15761 & protein \\
\hline 108 & Q1KUX5 & 6-phosphogluconate dehydrogenase, decarboxylating & C. spinosa & 20 & 176 & 0.18 & 6.06 & 53777 & OPP \\
\hline 109 & B3TLL5 & 40S ribosomal protein S19 & E. guineensis & 10 & 98 & 0.18 & 10.04 & 16202 & protein \\
\hline 110 & K3YZ28 & Uncharacterized protein & S. italica & 2 & 78 & 0.18 & 10.59 & 15971 & protein \\
\hline 111 & O04428 & Putative uncharacterized protein & C. paradisi & 7 & 146 & 0.18 & 5.59 & 32737 & biodegradation of xenobiotics. \\
\hline 112 & D7UPN0 & SEC13 family protein & L. japonicus & 5 & 100 & 0.17 & 6.01 & 32940 & protein \\
\hline 113 & Q84QC6 & Ubiquitin-conjugating enzyme & H. vulgare & 7 & 43 & 0.17 & 7.02 & 16667 & protein \\
\hline 114 & A5JVC6 & Putative uncharacterized protein & B. campestris & 3 & 54 & 0.17 & 6.25 & 34259 & cell wall \\
\hline 115 & K3ZSX0 & Uncharacterized protein & S. italica & 10 & 171 & 0.16 & 7.64 & 53564 & amino acid metabolism \\
\hline 116 & B3F8H6 & Sucrose synthase & N. langsdorffii & 36 & 453 & 0.16 & 6.40 & 92814 & major $\mathrm{CHO}$ metabolism \\
\hline 117 & Q4H1G2 & Methionine synthase & B. vulgaris & 58 & 926 & 0.16 & 5.59 & 88546 & amino acid metabolism \\
\hline 118 & A5C113 & Putative uncharacterized protein & V. vinifera & 4 & 90 & 0.16 & 6.46 & 17365 & fermentation \\
\hline 119 & E4MVY3 & mRNA, clone: RTFL01-01-M18 & T. halophila & 20 & 373 & 0.16 & 6.25 & 94734 & protein \\
\hline 120 & E4MVL4 & mRNA, clone: RTFL01-01-E20 & T. halophila & 7 & 126 & 0.16 & 5.58 & 54789 & protein \\
\hline 121 & K3YG24 & Uncharacterized protein & S. italica & 48 & 423 & 0.15 & 6.89 & 107135 & TCA \\
\hline 122 & A8MRE8 & Triosephosphate isomerase & A. thaliana & 6 & 95 & 0.15 & 7.49 & 32458 & photosynthesis \\
\hline 123 & F2DM28 & Predicted protein & H. vulgare & 8 & 84 & 0.15 & 7.33 & 38865 & biodegradation of xenobiotics. \\
\hline 124 & E4MX73 & mRNA, clone: RTFL01-10-N01 & T. halophila & 14 & 605 & 0.14 & 8.40 & 39684 & TCA \\
\hline 125 & C1IC54 & Cinnamyl alcohol dehydrogenase & G. hirsutum & 9 & 108 & 0.14 & 6.25 & 39574 & secondary metabolism \\
\hline 126 & A4UTS5 & Chloroplast ferredoxin-NADP+ reductase & P. sativum & 11 & 180 & 0.14 & 5.83 & 40392 & photosynthesis \\
\hline 127 & K4A7Q9 & Uncharacterized protein & S. italica & 13 & 197 & 0.14 & 5.74 & 60770 & protein \\
\hline
\end{tabular}




\begin{tabular}{|c|c|c|c|c|c|c|c|c|c|}
\hline 128 & E4MWY6 & Glucose-6-phosphate isomerase & T. halophila & 9 & 177 & 0.14 & 5.60 & 67312 & glycolysis \\
\hline 129 & Q9SH69 & 6-phosphogluconate dehydrogenase, decarboxylating & A. thaliana & 14 & 246 & 0.14 & 5.45 & 53686 & OPP \\
\hline 130 & D2XNF3 & Chaperone protein DnaK & P. patens & 16 & 125 & 0.14 & 5.50 & 75674 & stress \\
\hline 131 & F6H3T7 & Putative uncharacterized protein & V. vinifera & 15 & 94 & 0.14 & 5.97 & 40651 & not assigned \\
\hline 132 & B6UDL5 & Hydroxymethylbutenyl 4-diphosphate synthase & Z. mays & 35 & 505 & 0.14 & 6.11 & 82691 & secondary metabolism \\
\hline 133 & K4A7N1 & Uncharacterized protein & S. italica & 13 & 166 & 0.14 & 6.02 & 61055 & protein \\
\hline 134 & B6T6Q8 & Alcohol dehydrogenase class 3 & Z. mays & 10 & 212 & 0.14 & 7.02 & 41639 & misc \\
\hline 135 & E4MWP5 & mRNA, clone: RTFL01-15-G22 & T. halophila & 11 & 64 & 0.13 & 6.16 & 35258 & photosynthesis \\
\hline 136 & A0EJL8 & GDP-D-mannose-3',5'-epimerase & M. glabra & 14 & 137 & 0.13 & 6.21 & 42927 & redox \\
\hline 137 & K3YR06 & Uncharacterized protein & S. italica & 13 & 413 & 0.13 & 6.70 & 64225 & protein \\
\hline 138 & C0LQA1 & GDP-D-mannose-3',5'-epimerase & M. domestica & 13 & 114 & 0.13 & 6.16 & 42854 & redox \\
\hline 139 & K3ZU91 & Uncharacterized protein & S. italica & 6 & 92 & 0.13 & 8.70 & 42567 & protein \\
\hline 140 & A2PYH3 & Alpha chain of nascent polypeptide associated complex & N. benthamiana & 4 & 225 & 0.13 & 4.50 & 21911 & protein \\
\hline 141 & K4A7I4 & Uncharacterized protein & S. italica & 9 & 221 & 0.12 & 5.40 & 61666 & photosynthesis \\
\hline 142 & K3XZE3 & Uncharacterized protein & S. italica & 3 & 158 & 0.12 & 6.57 & 23153 & protein \\
\hline 143 & F2EL27 & Predicted protein & H. vulgare & 9 & 98 & 0.12 & 5.27 & 68608 & TCA \\
\hline 144 & Q0MYQ7 & Germin-like protein 2 & V. vinifera & 4 & 226 & 0.12 & 8.41 & 22832 & stress \\
\hline 145 & F2CW31 & Predicted protein & H. vulgare & 16 & 199 & 0.12 & 6.21 & 63075 & glycolysis \\
\hline 146 & Q93WQ1 & Dihydrolipoyl dehydrogenase & B. gymnorhiza & 9 & 88 & 0.12 & 7.17 & 54389 & TCA \\
\hline 147 & O78327 & Transketolase 1 & C. аппиит & 32 & 204 & 0.12 & 6.62 & 80398 & OPP \\
\hline 148 & E4MXI1 & mRNA, clone: RTFL01-39-B07 & T. halophila & 13 & 255 & 0.11 & 9.29 & 48063 & photosynthesis \\
\hline 149 & E5LCX1 & C14 cysteine protease & S. demissum & 5 & 283 & 0.11 & 6.93 & 23974 & protein \\
\hline 150 & B5LAU9 & Glutamine synthetase & C. аппиит & 22 & 152 & 0.11 & 6.98 & 47926 & N-metabolism \\
\hline 151 & B5LAU5 & DH putative B.-hydroxyacyl-ACP dehydratase & C. аппиит & 5 & 58 & 0.11 & 4.98 & 24080 & lipid metabolism \\
\hline 152 & B3TLP3 & Translation elongation factor EF-1 B. chain & E. guineensis & 6 & 209 & 0.11 & 4.69 & 24523 & protein \\
\hline 153 & D2KZ81 & S-adenosylmethionine synthase & M. domestica & 3 & 141 & 0.11 & 4.94 & 24831 & amino acid metabolism \\
\hline 154 & F2E397 & Predicted protein & H. vulgar & 10 & 247 & 0.11 & 5.87 & 34933 & major CHO metabolism \\
\hline 155 & F2DLC2 & Proteasome subunit alpha type & H. vulgare & 2 & 178 & 0.10 & 9.52 & 26087 & protein \\
\hline 156 & F2DP49 & Predicted protein & H. vulgare & 2 & 100 & 0.10 & 4.68 & 25913 & hormone metabolism \\
\hline 157 & F2CQY1 & Predicted protein & H. vulgare & 3 & 113 & 0.10 & 8.63 & 25529 & protein \\
\hline 158 & K3XZ10 & Uncharacterized protein & S. italica & 7 & 140 & 0.10 & 5.63 & 26286 & protein \\
\hline 159 & G8HAB2 & PLP-dependent aminotransferase & P. somniferum & 11 & 171 & 0.10 & 6.20 & 53501 & amino acid metabolism \\
\hline
\end{tabular}




\begin{tabular}{|c|c|c|c|c|c|c|c|c|c|}
\hline 160 & K4A8Y9 & Uncharacterized protein & S. italica & 6 & 194 & 0.10 & 7.61 & 53531 & TCA \\
\hline 161 & E1Z9U8 & Putative uncharacterized protein & C. variabilis & 2 & 102 & 0.10 & 5.67 & 27282 & protein \\
\hline 162 & Q5GAQ3 & Putative alanine aminotransferase & Z. mays & 17 & 213 & 0.09 & 6.54 & 57003 & amino acid metabolism \\
\hline 163 & A8MR12 & $26 \mathrm{~S}$ proteasome non-ATPase regulatory subunit 14 & A. thaliana & 6 & 75 & 0.09 & 5.64 & 29246 & protein \\
\hline 164 & A9PAK9 & Putative uncharacterized protein & P. trichocarpa & 8 & 115 & 0.09 & 6.89 & 50623 & amino acid metabolism \\
\hline 165 & B3TM23 & 40S ribosomal protein $\mathrm{S} 4$ & E. guineensis & 20 & 125 & 0.09 & 10.24 & 30023 & protein \\
\hline 166 & B6TNX7 & Proteasome subunit alpha type & Z. mays & 4 & 54 & 0.09 & 5.40 & 30582 & protein \\
\hline 167 & F2E2M4 & Predicted protein & H. vulgare & 13 & 244 & 0.09 & 5.29 & 92002 & stress \\
\hline 168 & E4MXR5 & mRNA, clone: RTFL01-40-M04 & T. halophila & 17 & 301 & 0.09 & 5.90 & 73489 & stress \\
\hline 169 & E5F725 & Putative uncharacterized protein & E. parvulum & 2 & 180 & 0.08 & 7.11 & 42041 & cell wall \\
\hline 170 & Q40270 & RNA-binding protein & M. crystallinum & 2 & 46 & 0.08 & 9.04 & 32001 & RNA \\
\hline 171 & B4FH62 & NAD-dependent epimerase/dehydratase & Z. mays & 7 & 68 & 0.08 & 8.82 & 31917 & not assigned \\
\hline 172 & F2CUK9 & Predicted protein & H. vulgare & 15 & 27 & 0.08 & 4.79 & 31275 & misc \\
\hline 173 & I0J3D8 & Proteasome subunit B. type & A. halleri & 4 & 98 & 0.08 & 8.54 & 33367 & protein \\
\hline 174 & F6HXK8 & Pectinesterase & V. vinifera & 6 & 92 & 0.08 & 6.06 & 69782 & cell wall \\
\hline 175 & В3H477 & Fumarate hydratase 2 & A. thaliana & 2 & 128 & 0.08 & 6.76 & 45707 & TCA \\
\hline 176 & E1ZNM5 & Putative uncharacterized protein & C. variabilis & 8 & 61 & 0.07 & 6.83 & 60332 & amino acid metabolism \\
\hline 177 & В6ТВ29 & Fructokinase-2 & Z. mays & 14 & 129 & 0.07 & 5.58 & 35875 & major $\mathrm{CHO}$ metabolism \\
\hline 178 & F2DAD8 & Predicted protein & H. vulgare & 6 & 49 & 0.07 & 7.15 & 50084 & amino acid metabolism \\
\hline 179 & Q0W9E2 & B. chaperonin 60 & S. commersonii & 5 & 66 & 0.07 & 7.27 & 63333 & protein \\
\hline 180 & Q39986 & Cysteine proteinase & Hemerocallis sp. & 4 & 163 & 0.07 & 8.18 & 40006 & protein \\
\hline 181 & B9RI89 & Serine-threonine protein kinase, plant-type, putative & R. communis & 2 & 71 & 0.07 & 6.05 & 40375 & not assigned \\
\hline 182 & E2FYC3 & Caffeic acid O-methyltransferase & C. sinensis & 8 & 101 & 0.07 & 5.83 & 40149 & secondary metabolism \\
\hline 183 & G5DWR0 & Branched-chain-amino-acid aminotransferase & S. latifolia & 4 & 33 & 0.07 & 5.50 & 38581 & amino acid metabolism \\
\hline 184 & $\mathrm{O} 24135$ & Citrate synthase & N. tabacum & 6 & 108 & 0.07 & 7.85 & 52744 & TCA \\
\hline 185 & K3XWI6 & Uncharacterized protein & S. italica & 12 & 174 & 0.07 & 8.21 & 55489 & glycolysis \\
\hline 186 & F2D3S8 & Serine hydroxymethyltransferase & H. vulgare & 25 & 137 & 0.07 & 6.60 & 56215 & C1-metabolism \\
\hline 187 & Q948V5 & Mitochondrial processing peptidase alpha subunit & M. alba & 3 & 38 & 0.06 & 8.25 & 54978 & protein \\
\hline 188 & B4FYM0 & Acetyl-CoA acetyltransferase, cytosolic 2 & Z. mays & 8 & 351 & 0.06 & 4.88 & 43450 & secondary metabolism \\
\hline 189 & E5GBJ6 & Alcohol dehydrogenase & C. melo & 2 & 76 & 0.06 & 7.66 & 40658 & misc \\
\hline 190 & E5GCI7 & Ornithine carbamoyltransferase & C. melo & 3 & 123 & 0.06 & 8.47 & 42175 & amino acid metabolism \\
\hline 191 & A8I7T1 & Heat shock protein $90 \mathrm{~B}$ & C. reinhardtii & 10 & 169 & 0.06 & 8.40 & 87547 & stress \\
\hline
\end{tabular}




\begin{tabular}{|c|c|c|c|c|c|c|c|c|c|}
\hline 192 & B7SA66 & Hsp90-like protein & D. glomerata & 17 & 189 & 0.06 & 8.09 & 92918 & stress \\
\hline 193 & Q8H6B6 & Chaperone protein HtpG & X. viscosa & 20 & 179 & 0.06 & 9.36 & 92957 & stress \\
\hline 194 & A9P9A2 & Putative uncharacterized protein & P. trichocarpa & 15 & 169 & 0.06 & 4.97 & 46272 & RNA \\
\hline 195 & A7WPL0 & Putative reductase & N. tabacum & 13 & 142 & 0.06 & 6.60 & 44428 & TCA \\
\hline 196 & I1LCM5 & Uncharacterized protein & G. $\max$ & 4 & 65 & 0.06 & 8.32 & 47008 & TCA \\
\hline 197 & E4MVQ7 & mRNA, clone: RTFL01-03-C18 & T. halophila & 3 & 37 & 0.06 & 8.72 & 46631 & redox \\
\hline 198 & F2D642 & Predicted protein & H. vulgare & 5 & 40 & 0.06 & 7.77 & 44355 & not assigned \\
\hline 199 & F2DLJ7 & Predicted protein & H. vulgare & 2 & 147 & 0.06 & 6.96 & 44423 & hormone metabolism \\
\hline 200 & F2CSU5 & Predicted protein & H. vulgare & 4 & 60 & 0.06 & 5.02 & 45209 & amino acid metabolism \\
\hline 201 & H9A1W2 & Acyl-activating enzyme 10 & C. sativa & 4 & 125 & 0.06 & 6.49 & 62013 & lipid metabolism \\
\hline 202 & I6YMA7 & Uncharacterized protein & L. usitatissimum & 7 & 34 & 0.06 & 6.39 & 89937 & signalling \\
\hline 203 & F2DGF3 & Predicted protein & H. vulgare & 7 & 94 & 0.05 & 6.81 & 115601 & TCA \\
\hline 204 & Q93YG1 & Monodehydroascorbate reductase & M. crystallinum & 5 & 113 & 0.05 & 6.54 & 51944 & redox \\
\hline 205 & F2E941 & Predicted protein & H. vulgare & 8 & 140 & 0.05 & 6.67 & 52201 & protein \\
\hline 206 & B9HJ23 & Predicted protein & P. trichocarpa & 6 & 123 & 0.05 & 7.09 & 101700 & misc \\
\hline 207 & G8HAB1 & PLP-dependent aminotransferase & P. somniferum & 6 & 71 & 0.05 & 5.96 & 50587 & secondary metabolism \\
\hline 208 & Q8VWP8 & Acyltransferase-like protein & G. hirsutum & 3 & 103 & 0.05 & 6.81 & 48286 & not assigned \\
\hline 209 & F2DIS4 & Malic enzyme & H. vulgare & 4 & 35 & 0.05 & 7.40 & 68411 & TCA \\
\hline 210 & B2KNE6 & Phospholipase D & H. annuus & 15 & 131 & 0.05 & 5.52 & 92315 & lipid metabolism \\
\hline 211 & Q1HG95 & Sucrose synthase & V. album & 15 & 50 & 0.05 & 6.09 & 92607 & major $\mathrm{CHO}$ metabolism \\
\hline 212 & K3YRV8 & Uncharacterized protein & S. italica & 5 & 78 & 0.05 & 6.92 & 55802 & TCA \\
\hline 213 & Q38766 & Victorin binding protein & A. sativa & 11 & 142 & 0.05 & 8.28 & 112298 & photosynthesis \\
\hline 214 & H9C956 & 3-deoxy-D-arabino-heptulosonate 7-phosphate synthase & N. benthamiana & 5 & 98 & 0.05 & 7.27 & 53191 & amino acid metabolism \\
\hline 215 & Q9S768 & Alanine aminotransferase & O. sativa & 4 & 201 & 0.05 & 6.79 & 53130 & amino acid metabolism \\
\hline 216 & K4A938 & Uncharacterized protein & S. italica & 5 & 63 & 0.05 & 6.96 & 53547 & nucleotide metabolism \\
\hline 217 & B6THZ8 & Threonine synthase & Z. mays & 2 & 58 & 0.05 & 6.65 & 58091 & amino acid metabolism \\
\hline 218 & Q2LFC1 & AGO4-2 & N. benthamiana & 9 & 92 & 0.04 & 8.78 & 102331 & RNA \\
\hline 219 & B5BLW2 & Carotenoid cleavage dioxygenase 1 & M. truncatula & 4 & 43 & 0.04 & 8.76 & 61063 & secondary metabolism \\
\hline 220 & B1NYI4 & Phytoene desaturase & N. benthamiana & 5 & 65 & 0.04 & 6.74 & 65942 & secondary metabolism \\
\hline 221 & B2Z6P0 & 4-Coumarate:CoA ligase & P. trichocarpa & 3 & 63 & 0.04 & 5.69 & 59541 & secondary metabolism \\
\hline 222 & Q9ZVV4 & At1g55570/T5A14_1 & A. thaliana & 5 & 66 & 0.04 & 6.79 & 62687 & misc \\
\hline 223 & A8MQH4 & Methylmalonate-semialdehyde dehydrogenase & A. thaliana & 2 & 90 & 0.04 & 8.91 & 65229 & amino acid metabolism \\
\hline
\end{tabular}




\begin{tabular}{|c|c|c|c|c|c|c|c|c|c|}
\hline 224 & B6TLT1 & Serine/threonine-protein phosphatase $2 \mathrm{~A} 65 \mathrm{kDa}$ regulatory subunit $\mathrm{AB}$. isoform & Z. mays & 2 & 40 & 0.04 & 5.03 & 66318 & protein \\
\hline 225 & K4A6V8 & Uncharacterized protein & S. italica & 9 & 125 & 0.04 & 8.70 & 70549 & glycolysis \\
\hline 226 & F2DAP7 & Malic enzyme & H. vulgare & 7 & 172 & 0.04 & 5.78 & 69436 & TCA \\
\hline 227 & F2CRY1 & Predicted protein & H. vulgare & 3 & 34 & 0.04 & 7.88 & 69011 & misc \\
\hline 228 & J9Z414 & B.-D-glucan exohydolase & E. guineensis & 2 & 97 & 0.04 & 7.03 & 69462 & cell wall \\
\hline 229 & Q6L619 & B.-galactosidase & R. sativus & 4 & 78 & 0.04 & 8.38 & 93664 & misc \\
\hline 230 & Q8L5C2 & $110 \mathrm{kDa} 4 \mathrm{SNc}-$ Tudor domain protein & P. sativum & 4 & 30 & 0.03 & 7.36 & 108600 & RNA \\
\hline 231 & K3YKV0 & Uncharacterized protein & S. italica & 13 & 169 & 0.03 & 5.54 & 87462 & cell \\
\hline 232 & K3YQC4 & Uncharacterized protein & S. italica & 7 & 49 & 0.03 & 6.33 & 78439 & secondary metabolism \\
\hline 233 & Q9XFX7 & Phospholipase D & C. plantagineum & 7 & 31 & 0.03 & 6.16 & 92228 & lipid metabolism \\
\hline 234 & B6U237 & Heat shock $70 \mathrm{kDa}$ protein 4 & Z. mays & 8 & 109 & 0.03 & 5.43 & 94309 & stress \\
\hline 235 & Q9XFX8 & Phospholipase D & C. plantagineum & 3 & 99 & 0.03 & 5.66 & 92155 & lipid metabolism \\
\hline 236 & A5AW47 & Profilin & $V$. vinifera & 26 & 81 & 0.03 & 5.44 & 92852 & cell \\
\hline 237 & E4MXV2 & mRNA, clone: RTFL01-41-F15 & T. halophila & 12 & 70 & - & 6.09 & 64768 & secondary metabolism \\
\hline
\end{tabular}

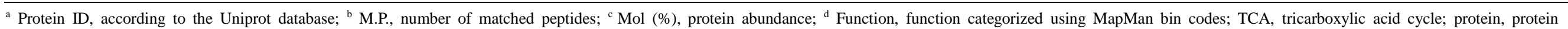

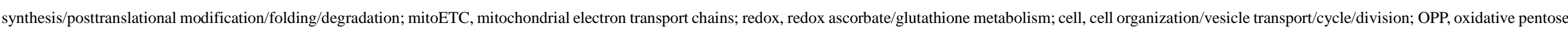
phosphate; DNA, DNA synthesis/repair; RNA, RNA processing/regulation of transcription; and misc, miscellaneous. 
Supplemental Table 5. Proteins Identified in L. japonica Immature Flower Buds at Fraction of Elution of pH 9 using Combinatorial Peptide Ligand Libraries Fractionation.

\begin{tabular}{|c|c|c|c|c|c|c|c|c|c|}
\hline No. & Protein ID ${ }^{a}$ & Description & Species & M.P. ${ }^{b}$ & Score & $\operatorname{Mol}(\%)^{\mathrm{c}}$ & $\mathrm{pI}$ & Mass (Da) & Function $^{\mathrm{d}}$ \\
\hline 1 & C4B8E5 & Glyceraldehyde-3-phosphate dehydrogenase & T. gesneriana & 109 & 3559 & 1.80 & 7.96 & 20964 & glycolysis \\
\hline 2 & Q3LGW4 & Ribulose-1,5-bisphosphate carboxylase/oxygenase large subunit & F. benjamina & 12 & 166 & 1.15 & 4.67 & 9052 & photosynthesis \\
\hline 3 & D0F109 & Ribulose bisphosphate carboxylase large chain & S. dariensis & 36 & 269 & 1.15 & 7.68 & 19294 & photosynthesis \\
\hline 4 & Q9GD57 & Ribulose 5-bisphosphate carboxylase, large subunit & P. yemenensis & 170 & 1291 & 1.11 & 6.89 & 51857 & photosynthesis \\
\hline 5 & H1ZY49 & Translation elongation factor 1-alpha & L. maackii & 37 & 289 & 1.11 & 5.00 & 11619 & protein \\
\hline 6 & Q8MCW6 & Ribulose-1,5-bisphosphate carboxylase large subunit & M. racemosa & 160 & 1680 & 1.00 & 6.58 & 51631 & photosynthesis \\
\hline 7 & $\mathrm{O} 03843$ & Ribulose-bisphosphate carboxylase & T. glandulifera & 133 & 1566 & 0.89 & 6.04 & 53346 & photosynthesis \\
\hline 8 & B3TLP4 & Ribosomal protein L30 & E. guineensis & 7 & 132 & 0.84 & 9.72 & 12451 & protein \\
\hline 9 & Q6PKU2 & Putative glyceraldehyde-3-phosphate dehydrogenase & O. minor & 59 & 1614 & 0.74 & 7.09 & 22122 & glycolysis \\
\hline 10 & Q5KTY2 & Ribulose-1,5-bisphosphate carboxylase large subunit & A. sutepensis & 93 & 836 & 0.72 & 6.92 & 51143 & photosynthesis \\
\hline 11 & Q40463 & NTGB2 & N. tabacum & 6 & 135 & 0.70 & 6.19 & 15827 & signalling \\
\hline 12 & J9QAL9 & Ribosomal protein $\mathrm{L} 2$ & A. corniculatum & 11 & 161 & 0.68 & 10.23 & 21703 & protein \\
\hline 13 & Q9FX54 & At1g13440/F13B4_8 & A. thaliana & 112 & 3676 & 0.63 & 7.18 & 37004 & glycolysis \\
\hline 14 & Q9FEM4 & Catalase & B. pendula & 18 & 290 & 0.62 & 5.48 & 17247 & redox \\
\hline 15 & D2CJB9 & ATP synthase subunit beta & B. multifida & 26 & 671 & 0.61 & 5.05 & 51275 & photosynthesis \\
\hline 16 & A7KQH2 & Beta-tubulin & E. grandis & 59 & 784 & 0.61 & 4.84 & 50255 & cell \\
\hline 17 & Q9TKH9 & Ribulose 1,5-bisphosphate carboxylase large subunit & H. albomarginata & 84 & 861 & 0.60 & 6.37 & 51833 & photosynthesis \\
\hline 18 & Q8MCX1 & Ribulose-1,5-bisphosphate carboxylase large subunit & C. axillaris & 86 & 754 & 0.60 & 6.58 & 52386 & photosynthesis \\
\hline 19 & E5GBV9 & Glyceraldehyde-3-phosphate dehydrogenase & C. melo & 109 & 2148 & 0.59 & 6.62 & 36471 & glycolysis \\
\hline 20 & J3RTS9 & Glyceraldehyde-3-phosphate dehydrogenase & C. pentagona & 122 & 3463 & 0.58 & 7.23 & 32382 & glycolysis \\
\hline 21 & Q6Y1C4 & $40 \mathrm{~S}$ ribosomal protein $\mathrm{S} 9$ & L. saligna & 23 & 58 & 0.58 & 10.95 & 14138 & protein \\
\hline 22 & B3TM39 & Ribosomal protein S14 & E. guineensis & 6 & 89 & 0.57 & 10.70 & 16428 & protein \\
\hline 23 & D9ZC79 & Malate dehydrogenase & S. globosa & 8 & 213 & 0.56 & 5.02 & 12369 & TCA \\
\hline 24 & Q5ZF83 & Putative uncharacterized protein & P. major & 26 & 420 & 0.55 & 7.28 & 25390 & cell wall \\
\hline 25 & Q08II7 & Heat shock protein 70 -like protein & L. sativa & 6 & 183 & 0.54 & 10.33 & 12539 & stress \\
\hline 26 & B0M1B1 & Peroxisomal glycolate oxidase & G. $\max$ & 35 & 399 & 0.54 & 9.13 & 40825 & photosynthesis \\
\hline 27 & B3H7J6 & $40 \mathrm{~S}$ ribosomal protein $\mathrm{S} 9-1$ & A. thaliana & 27 & 202 & 0.54 & 9.80 & 19090 & protein \\
\hline 28 & Q4H1G1 & Adenosylhomocysteinase & B. vulgaris & 52 & 460 & 0.54 & 6.19 & 54052 & amino acid metabolism \\
\hline 29 & Q9XEW9 & Elongation factor 1-alpha & L. longiflorum & 61 & 388 & 0.54 & 9.07 & 49711 & protein \\
\hline 30 & Q6H3X8 & $14-3-3$ protein isoform $16 \mathrm{R}$ & S. tuberosum & 15 & 254 & 0.53 & 4.78 & 29032 & signalling \\
\hline 31 & A9PD17 & Predicted protein & P. trichocarpa & 45 & 765 & 0.48 & 5.82 & 47103 & protein \\
\hline
\end{tabular}




\begin{tabular}{|c|c|c|c|c|c|c|c|c|c|}
\hline 32 & Q84RD8 & Adenosylhomocysteinase & M. truncatula & 53 & 541 & 0.48 & 6.01 & 53790 & amino acid metabolism \\
\hline 33 & B6EBD6 & Heat shock protein $90-2$ & G. $\max$ & 70 & 808 & 0.46 & 5.07 & 80392 & stress \\
\hline 34 & G1DVA7 & Non-specific lipid-transfer protein & H. annиus & 12 & 52 & 0.46 & 8.41 & 12193 & lipid metabolism \\
\hline 35 & B6SZ69 & Heat shock cognate $70 \mathrm{kDa}$ protein 2 & Z. mays & 43 & 712 & 0.45 & 5.19 & 71493 & stress \\
\hline 36 & F2CT86 & Predicted protein & H. vulgare & 6 & 139 & 0.45 & 7.47 & 22104 & signalling \\
\hline 37 & Q39640 & Glycolate oxidase & C. Kurokawa & 30 & 334 & 0.44 & 8.79 & 40345 & photosynthesis \\
\hline 38 & B6UGQ7 & $60 \mathrm{~S}$ ribosomal protein $\mathrm{L} 23$ & Z. mays & 19 & 405 & 0.43 & 10.39 & 15232 & protein \\
\hline 39 & Q9M6B3 & Malate dehydrogenase & V. vinifera & 11 & 329 & 0.42 & 8.62 & 37137 & TCA \\
\hline 40 & F2EG92 & Predicted protein & H. vulgare & 4 & 87 & 0.41 & 12.12 & 7602 & protein \\
\hline 41 & $\mathrm{O} 22331$ & Glutamine synthetase & H. brasiliensis & 27 & 291 & 0.41 & 6.20 & 39504 & $\mathrm{~N}$-metabolism \\
\hline 42 & E1AXT8 & Glycolate oxidase & N. benthamiana & 19 & 128 & 0.41 & 9.00 & 40627 & photosynthesis \\
\hline 43 & A2V880 & G protein beta-subunit-like protein & N. tabacum & 12 & 234 & 0.39 & 7.09 & 16100 & signalling \\
\hline 44 & B3TLN9 & Cytoplasmic ribosomal protein $\mathrm{S} 15 \mathrm{a}$ & E. guineensis & 8 & 82 & 0.38 & 9.89 & 14862 & protein \\
\hline 45 & Q8GZD5 & Xyloglucan endotransglycosylase & P. tremula & 52 & 537 & 0.37 & 7.75 & 34481 & cell wall \\
\hline 46 & A0ZQA9 & ATP synthase subunit beta & P. baccatum & 25 & 440 & 0.36 & 5.40 & 52897 & photosynthesis \\
\hline 47 & D0EJY9 & Molecular chaperone Hsp90-3 & N. benthamiana & 55 & 637 & 0.36 & 5.02 & 80638 & stress \\
\hline 48 & K4ACE3 & Uncharacterized protein & S. italica & 26 & 630 & 0.35 & 9.47 & 35803 & TCA \\
\hline 49 & K3Z913 & Uncharacterized protein & S. italica & 19 & 304 & 0.35 & 6.09 & 26793 & cell \\
\hline 50 & A8J597 & Ribosomal protein L12 & C. reinhardtii & 11 & 119 & 0.35 & 9.06 & 17822 & protein \\
\hline 51 & Q6T379 & Triosephosphate isomerase & S. chacoense & 9 & 211 & 0.34 & 5.99 & 27251 & glycolysis \\
\hline 52 & F2DAU4 & Predicted protein & H. vulgare & 32 & 477 & 0.34 & 7.56 & 62696 & protein \\
\hline 53 & D0ELH5 & Peptidyl-prolyl cis-trans isomerase & C. sinensis & 13 & 261 & 0.34 & 8.46 & 18310 & cell \\
\hline 54 & O81830 & Putative uncharacterized protein AT4g27270 & A. thaliana & 7 & 121 & 0.33 & 6.79 & 22355 & lipid metabolism \\
\hline 55 & $\mathrm{O} 48903$ & Malate dehydrogenase & M. sativa & 23 & 719 & 0.32 & 8.29 & 38397 & gluconeogenesis \\
\hline 56 & F2E3I7 & Glutamine synthetase & H. vulgare & 22 & 212 & 0.31 & 5.48 & 39331 & $\mathrm{~N}$-metabolism \\
\hline 57 & Q2V3V9 & Putative peroxisomal (S)-2-hydroxy-acid oxidase 2 & A. thaliana & 14 & 302 & 0.31 & 8.80 & 40332 & photosynthesis \\
\hline 58 & Q7XTK9 & Catalase & P. persica & 31 & 649 & 0.30 & 7.25 & 57262 & redox \\
\hline 59 & Q5IA96 & Cytochrome oxidase subunit II & A. trichopoda & 2 & 88 & 0.30 & 4.75 & 9794 & mitoETC \\
\hline 60 & D7NHW9 & 2-phospho-D-glycerate hydrolase & P. trifoliata & 24 & 503 & 0.30 & 5.78 & 47986 & glycolysis \\
\hline 61 & Q1WFH5 & Phosphoenolpyruvate carboxylase isoform 3 & C. hilariana & 18 & 125 & 0.29 & 8.94 & 41462 & glycolysis \\
\hline 62 & K3XFR6 & Uncharacterized protein & S. italica & 36 & 493 & 0.29 & 7.46 & 66448 & TCA \\
\hline 63 & K3XXC5 & Uncharacterized protein & S. italica & 16 & 273 & 0.29 & 7.06 & 42409 & glycolysis \\
\hline
\end{tabular}




\begin{tabular}{|c|c|c|c|c|c|c|c|c|c|}
\hline 64 & B2VQE0 & Methionine synthase & O. ramosa & 37 & 430 & 0.29 & 6.40 & 84862 & amino acid metabolism \\
\hline 65 & D6PPS9 & AT3G02360-like protein & C. grandiflora & 12 & 177 & 0.29 & 9.22 & 21022 & OPP \\
\hline 66 & B6SJZ8 & Glyceraldehyde-3-phosphate dehydrogenase, cytosolic & Z. mays & 71 & 2090 & 0.28 & 6.96 & 36614 & glycolysis \\
\hline 67 & F2CQ27 & Predicted protein & H. vulgare & 9 & 105 & 0.28 & 6.66 & 25460 & signalling \\
\hline 68 & B3TM23 & $40 \mathrm{~S}$ ribosomal protein $\mathrm{S} 4$ & E. guineensis & 24 & 267 & 0.27 & 10.24 & 30023 & protein \\
\hline 69 & A8MR50 & $60 \mathrm{~S}$ ribosomal protein $\mathrm{L} 34-1$ & A. thaliana & 4 & 97 & 0.26 & 11.84 & 11023 & cell wall \\
\hline 70 & F2CQQ3 & Predicted protein & H. vulgare & 17 & 467 & 0.26 & 5.16 & 54310 & transport \\
\hline 71 & Q9SW73 & Isocitrate dehydrogenase [NADP] & C. limon & 11 & 190 & 0.25 & 6.95 & 46823 & TCA \\
\hline 72 & K3XZA2 & Uncharacterized protein & S. italica & 17 & 236 & 0.25 & 9.58 & 23221 & cell \\
\hline 73 & $\mathrm{O} 24511$ & Catalase & N. tabacum & 23 & 324 & 0.24 & 6.86 & 57225 & redox \\
\hline 74 & Q9SXR9 & LeArcA1 protein & S. lycopersicum & 29 & 518 & 0.24 & 7.12 & 36363 & signalling \\
\hline 75 & B3TLL5 & 40S ribosomal protein S19 & E. guineensis & 21 & 47 & 0.24 & 10.04 & 16202 & protein \\
\hline 76 & F8WLB7 & $40 \mathrm{~S}$ ribosomal protein $\mathrm{S} 3$ & C. unshiu & 4 & 106 & 0.24 & 9.63 & 20599 & protein \\
\hline 77 & B5TM95 & NADH dehydrogenase subunit 7 & A. thaliana & 11 & 217 & 0.24 & 7.12 & 45555 & mitoETC \\
\hline 78 & E4MXI2 & mRNA, clone: RTFL01-39-D20 & T. halophila & 34 & 462 & 0.24 & 5.21 & 73890 & stress \\
\hline 79 & K3Z5U9 & Uncharacterized protein & S. italica & 19 & 296 & 0.24 & 5.48 & 49826 & photosynthesis \\
\hline 80 & F2DAC4 & Predicted protein & H. vulgare & 3 & 53 & 0.24 & 7.18 & 12136 & not assigned \\
\hline 81 & D7SY81 & Putative uncharacterized protein & V. vinifera & 4 & 66 & 0.24 & 6.47 & 12099 & stress \\
\hline 82 & B6T6Q5 & GAST1 protein & Z. mays & 8 & 35 & 0.23 & 9.10 & 12384 & hormone metabolism \\
\hline 83 & Q0VYL5 & GASA & F. sylvatica & 5 & 39 & 0.22 & 9.03 & 12634 & hormone metabolism \\
\hline 84 & $\mathrm{O} 23254$ & Serine hydroxymethyltransferase & A. thaliana & 20 & 496 & 0.22 & 7.23 & 52141 & C1-metabolism \\
\hline 85 & A1BLP6 & Thioredoxin & M. truncatula & 7 & 70 & 0.22 & 6.16 & 12904 & redox \\
\hline 86 & Q9ATF4 & Ribosomal protein L33 & C. sativa & 2 & 107 & 0.22 & 10.58 & 12831 & protein \\
\hline 87 & Q9AXR6 & ATP:citrate lyase & C. аппиит & 31 & 420 & 0.22 & 7.39 & 66376 & TCA \\
\hline 88 & Q8LG68 & UDP-glucose 6-dehydrogenase & A. thaliana & 23 & 503 & 0.22 & 6.33 & 53591 & stress \\
\hline 89 & $\mathrm{~A} 2 \mathrm{PZC} 2$ & UDP-Glucose:protein transglucosylase & C. reinhardtii & 10 & 130 & 0.22 & 6.18 & 39846 & cell wall \\
\hline 90 & Q9LF33 & UDP-glucose 6-dehydrogenase & A. thaliana & 16 & 497 & 0.22 & 6.04 & 53653 & cell wall \\
\hline 91 & G5DWK9 & 3-dehydroquinate synthase & S. latifolia & 2 & 37 & 0.22 & 7.03 & 13075 & $\begin{array}{l}\text { co-factor and vitamine } \\
\text { metabolism }\end{array}$ \\
\hline 92 & K4A9V6 & Uncharacterized protein & S. italica & 30 & 353 & 0.21 & 5.10 & 50519 & cell \\
\hline 93 & Q52UN0 & Peptidyl-prolyl cis-trans isomerase & C. sativus & 13 & 87 & 0.21 & 8.44 & 18340 & cell \\
\hline 94 & A8MRE8 & Triosephosphate isomerase & A. thaliana & 8 & 136 & 0.21 & 7.49 & 32458 & photosynthesis \\
\hline 95 & G5DVX2 & Phosphoglycerate kinase & S. latifolia & 29 & 423 & 0.20 & 6.58 & 51352 & photosynthesis \\
\hline
\end{tabular}




\begin{tabular}{|c|c|c|c|c|c|c|c|c|c|}
\hline 96 & K3ZTJ3 & Uncharacterized protein & S. italica & 17 & 474 & 0.20 & 8.94 & 47124 & photosynthesis \\
\hline 97 & Q9SRV5 & 5-methyltetrahydropteroyltriglutamate--homocysteine methyltransferase & A. thaliana & 41 & 400 & 0.20 & 6.51 & 84873 & amino acid metabolism \\
\hline 98 & A0EJL8 & GDP-D-mannose-3',5'-epimerase & M. glabra & 21 & 255 & 0.20 & 6.21 & 42927 & redox \\
\hline 99 & G5DXP1 & Fructose-bisphosphate aldolase & S. latifolia & 10 & 311 & 0.20 & 5.58 & 42771 & photosynthesis \\
\hline 100 & Q9SXX4 & Fructose-bisphosphate aldolase & N. paniculata & 10 & 247 & 0.20 & 6.16 & 42832 & photosynthesis \\
\hline 101 & E4MW44 & mRNA, clone: RTFL01-05-I15 & T. halophila & 13 & 157 & 0.20 & 7.33 & 42078 & cell \\
\hline 102 & B5LAU9 & Glutamine synthetase & C. аппиит & 15 & 75 & 0.20 & 6.93 & 47926 & N-metabolism \\
\hline 103 & B5LAU5 & DH putative beta-hydroxyacyl-ACP dehydratase & C. аппиит & 6 & 52 & 0.20 & 9.29 & 24080 & lipid metabolism \\
\hline 104 & A9P8Q7 & Predicted protein & P. trichocarpa & 17 & 249 & 0.20 & 4.84 & 28824 & signalling \\
\hline 105 & B4FPK8 & Cytochrome b5 & Z. mays & 4 & 81 & 0.19 & 5.30 & 14958 & redox \\
\hline 106 & K3ZW60 & Uncharacterized protein & S. italica & 19 & 275 & 0.19 & 9.76 & 29913 & protein \\
\hline 107 & K3ZUR5 & Uncharacterized protein & S. italica & 7 & 137 & 0.19 & 6.24 & 36749 & $\begin{array}{l}\text { co-factor and vitamine } \\
\text { metabolism }\end{array}$ \\
\hline 108 & A8MQR4 & $60 \mathrm{~S}$ acidic ribosomal protein $\mathrm{P} 0-2$ & A. thaliana & 5 & 84 & 0.18 & 4.84 & 30656 & protein \\
\hline 109 & F2D448 & Predicted protein & H. vulgare & 15 & 221 & 0.18 & 10.17 & 30607 & protein \\
\hline 110 & E4MVY3 & mRNA, clone: RTFL01-01-M18 & T. halophila & 35 & 347 & 0.18 & 6.25 & 94734 & protein \\
\hline 111 & K3YQW5 & Uncharacterized protein & S. italica & 14 & 286 & 0.18 & 5.39 & 68637 & transport \\
\hline 112 & E5GBS0 & 40 S ribosomal protein S24 & C. melo & 6 & 131 & 0.18 & 10.64 & 15761 & protein \\
\hline 113 & F2D861 & Predicted protein & H. vulgare & 12 & 480 & 0.18 & 10.43 & 15765 & protein \\
\hline 114 & B9N744 & Gamma class glutathione transferase EF1Bgamma3 & P. trichocarpa & 17 & 298 & 0.17 & 6.05 & 48353 & protein \\
\hline 115 & K3YZ28 & Uncharacterized protein & S. italica & 5 & 128 & 0.17 & 10.59 & 15971 & protein \\
\hline 116 & Q6SKP4 & Ribosomal protein L3 & S. lycopersicum & 17 & 96 & 0.17 & 10.08 & 44836 & protein \\
\hline 117 & K4A7I4 & Uncharacterized protein & S. italica & 13 & 348 & 0.17 & 5.40 & 61666 & photosynthesis \\
\hline 118 & B6THG9 & $60 \mathrm{~S}$ ribosomal protein $\mathrm{L} 5-1$ & Z. mays & 11 & 325 & 0.16 & 9.32 & 34462 & protein \\
\hline 119 & В6Т3Р9 & Enolase & Z. mays & 10 & 352 & 0.15 & 5.82 & 48386 & glycolysis \\
\hline 120 & O81609 & Nodule-enhanced malate dehydrogenase & P. sativum & 6 & 183 & 0.15 & 7.11 & 42106 & TCA \\
\hline 121 & E5F725 & Putative uncharacterized protein & E. parvulum & 3 & 120 & 0.15 & 7.75 & 42041 & cell wall \\
\hline 122 & E5GCD6 & Multicopper oxidase & C. melo & 34 & 153 & 0.15 & 9.32 & 60810 & not assigned \\
\hline 123 & B3TLY3 & Ribosomal protein L15 & E. guineensis & 6 & 297 & 0.15 & 11.49 & 24371 & protein \\
\hline 124 & Q8LC80 & Putative calcium-binding protein, calreticulin & A. thaliana & 8 & 195 & 0.15 & 4.53 & 48385 & signalling \\
\hline 125 & F2D1A7 & Predicted protein & H. vulgare & 31 & 309 & 0.15 & 4.53 & 17886 & protein \\
\hline 126 & A8MRF3 & $60 \mathrm{~S}$ ribosomal protein L18a-1 & A. thaliana & 4 & 100 & 0.15 & 9.73 & 36337 & misc \\
\hline 127 & A2V884 & Translationally controlled tumor protein like protein & N. tabacum & 4 & 46 & 0.15 & 9.80 & 18052 & development \\
\hline
\end{tabular}




\begin{tabular}{|c|c|c|c|c|c|c|c|c|c|}
\hline 128 & D3GQL1 & Aconitate hydratase 3 & C. clementina & 35 & 384 & 0.15 & 6.30 & 98669 & TCA \\
\hline 129 & K3ZA87 & Uncharacterized protein & S. italica & 5 & 42 & 0.15 & 8.46 & 18363 & stress \\
\hline 130 & D6PAY2 & Peptidyl-prolyl cis-trans isomerase & V. hybrid cultivar & 9 & 215 & 0.15 & 8.87 & 18582 & cell \\
\hline 131 & Q645M9 & Glyoxisomal malate dehydrogenase & S. lycopersicum & 12 & 326 & 0.15 & 7.94 & 38007 & gluconeogenesis \\
\hline 132 & Q4H1G2 & Methionine synthase & B. vulgaris & 36 & 575 & 0.15 & 6.46 & 88546 & amino acid metabolism \\
\hline 133 & K3ZA95 & Uncharacterized protein & S. italica & 38 & 94 & 0.14 & 5.22 & 19471 & photosynthesis \\
\hline 134 & K3XGJ6 & Uncharacterized protein & S. italica & 10 & 140 & 0.14 & 8.53 & 58129 & protein \\
\hline 135 & F2DTH4 & Adenylate kinase & H. vulgare & 4 & 69 & 0.14 & 7.81 & 26477 & nucleotide metabolism \\
\hline 136 & K3Z566 & Uncharacterized protein & S. italica & 16 & 243 & 0.14 & 7.77 & 59005 & C1-metabolism \\
\hline 137 & B3F8H6 & Sucrose synthase & N. langsdorffii & 34 & 528 & 0.14 & 6.40 & 92814 & major $\mathrm{CHO}$ metabolism \\
\hline 138 & K3ZVI3 & Uncharacterized protein & S. italica & 11 & 80 & 0.14 & 5.16 & 33393 & protein \\
\hline 139 & Q9ZNX1 & NAD-dependent isocitrate dehydrogenase & N. tabacum & 3 & 83 & 0.13 & 7.52 & 40651 & TCA \\
\hline 140 & B6UDN1 & Fructose-bisphosphate aldolase & Z. mays & 9 & 173 & 0.13 & 7.96 & 41956 & photosynthesis \\
\hline 141 & F2DTL1 & Fructose-bisphosphate aldolase & H. vulgare & 9 & 185 & 0.13 & 8.35 & 41916 & photosynthesis \\
\hline 142 & B3TLQ5 & $60 \mathrm{~S}$ ribosomal protein $\mathrm{L} 11$ & E. guineensis & 10 & 158 & 0.13 & 9.92 & 20950 & protein \\
\hline 143 & K3YR06 & Uncharacterized protein & S. italica & 13 & 239 & 0.13 & 6.16 & 64225 & protein \\
\hline 144 & A0FKE6 & Chloroplast threonine deaminase 1 & S. lycopersicum & 6 & 193 & 0.12 & 6.37 & 66653 & amino acid metabolism \\
\hline 145 & Q1KUM7 & Putative uncharacterized protein & C. spinosa & 7 & 133 & 0.12 & 6.62 & 59525 & protein \\
\hline 146 & B4FRS3 & Uncharacterized protein & Z. mays & 3 & 31 & 0.12 & 8.06 & 22134 & not assigned \\
\hline 147 & H9C956 & 3-deoxy-D-arabino-heptulosonate 7-phosphate synthase & N. benthamiana & 17 & 217 & 0.12 & 8.28 & 53191 & amino acid metabolism \\
\hline 148 & Q0MYQ7 & Germin-like protein 2 & V. vinifera & 3 & 133 & 0.12 & 9.36 & 22832 & stress \\
\hline 149 & A9P9A2 & Putative uncharacterized protein & P. trichocarpa & 24 & 332 & 0.12 & 8.41 & 46272 & RNA \\
\hline 150 & Q9STA4 & Cysteine protease & M. sativa & 2 & 30 & 0.12 & 5.17 & 22875 & protein \\
\hline 151 & Q1EMR1 & Nucleoside-diphopshate-sugar dehydratase & P. major & 10 & 44 & 0.12 & 5.57 & 22710 & cell wall \\
\hline 152 & K3ZSX0 & Uncharacterized protein & S. italica & 6 & 124 & 0.12 & 7.64 & 53564 & amino acid metabolism \\
\hline 153 & K3XZE3 & Uncharacterized protein & S. italica & 2 & 101 & 0.12 & 5.27 & 23153 & protein \\
\hline 154 & A9PE52 & Gamma class glutathione transferase EF1Bgamma2 & P. trichocarpa & 13 & 139 & 0.11 & 5.80 & 48127 & protein \\
\hline 155 & E5LCX1 & $\mathrm{C} 14$ cysteine protease & S. demissum & 5 & 127 & 0.11 & 8.67 & 23974 & protein \\
\hline 156 & $\mathrm{~K} 3 \mathrm{XX} 30$ & Uncharacterized protein & S. italica & 9 & 73 & 0.11 & 4.98 & 47991 & protein \\
\hline 157 & B6SVI5 & Putative uncharacterized protein & Z. mays & 9 & 39 & 0.11 & 5.07 & 24337 & not assigned \\
\hline 158 & F2DGF3 & Predicted protein & H. vulgare & 14 & 376 & 0.11 & 6.81 & 115601 & TCA \\
\hline 159 & B3TLP3 & Translation elongation factor EF-1 beta chain & E. guineensis & 4 & 94 & 0.11 & 4.69 & 24523 & protein \\
\hline
\end{tabular}




\begin{tabular}{|c|c|c|c|c|c|c|c|c|c|}
\hline 160 & K3Y9Y1 & Uncharacterized protein & S. italica & 2 & 55 & 0.11 & 10.07 & 24396 & protein \\
\hline 161 & B6TPE4 & Glutamate-1-semialdehyde 2,1-aminomutase & Z. mays & 6 & 62 & 0.11 & 6.55 & 50223 & tetrapyrrole synthesis \\
\hline 162 & E5GCQ7 & Glutamate-1-semialdehyde 2,1-aminomutase & C. melo & 6 & 158 & 0.11 & 6.61 & 50339 & tetrapyrrole synthesis \\
\hline 163 & Q6R9J5 & ATPase subunit 4 & Z. mays & 4 & 48 & 0.11 & 8.48 & 24941 & mitoETC \\
\hline 164 & F2DBE0 & Predicted protein & H. vulgare & 4 & 40 & 0.10 & 8.22 & 34595 & TCA \\
\hline 165 & Q9FZG9 & Putative pectinesterase & A. thaliana & 18 & 139 & 0.10 & 9.45 & 60580 & cell wall \\
\hline 166 & A7BG58 & 2-C-methyl-D-erythritol 2,4-cyclodiphosphate synthase & C. jambhiri & 3 & 38 & 0.10 & 5.48 & 25632 & secondary metabolism \\
\hline 167 & A2Y926 & Proteasome subunit beta type & O. sativa & 2 & 63 & 0.10 & 8.78 & 26357 & protein \\
\hline 168 & F2DLC2 & Proteasome subunit alpha type & H. vulgare & 2 & 169 & 0.10 & 4.68 & 26087 & protein \\
\hline 169 & F2DP49 & Predicted protein & H. vulgare & 5 & 53 & 0.10 & 8.63 & 25913 & hormone metabolism \\
\hline 170 & B9H5E3 & Predicted protein & P. trichocarpa & 19 & 126 & 0.10 & 9.01 & 61685 & not assigned \\
\hline 171 & Q9C6U3 & Putative uncharacterized protein T8G24.2 & A. thaliana & 6 & 120 & 0.10 & 7.59 & 34907 & not assigned \\
\hline 172 & Q40556 & Protein phosphatase $2 \mathrm{~A}$ & N. tabacum & 13 & 85 & 0.10 & 5.17 & 66079 & protein \\
\hline 173 & G8HAA9 & PLP-dependent aminotransferase & P. somniferum & 5 & 67 & 0.10 & 6.46 & 53606 & photosynthesis \\
\hline 174 & A8ASG2 & Phosphoenolpyruvate carboxylase & A. arborescens & 20 & 160 & 0.10 & 6.57 & 110813 & glycolysis \\
\hline 175 & C4NZX3 & Lipoxygenase & C. sinensis & 31 & 471 & 0.10 & 6.83 & 102365 & hormone metabolism \\
\hline 176 & Q5GAQ3 & Putative alanine aminotransferase & Z. mays & 5 & 85 & 0.09 & 5.64 & 57003 & amino acid metabolism \\
\hline 177 & $\mathrm{O} 23963$ & Elongation factor $\mathrm{Tu}$ & G. $\max$ & 9 & 174 & 0.09 & 5.14 & 38044 & protein \\
\hline 178 & Q2LFC3 & AGO1-2 & N. benthamiana & 14 & 236 & 0.09 & 9.31 & 109989 & development \\
\hline 179 & E3WHD5 & Lipoxygenase & V. hybrid cultivar & 31 & 519 & 0.09 & 6.55 & 100738 & hormone metabolism \\
\hline 180 & B6U0V6 & Endoplasmin & Z. mays & 13 & 289 & 0.09 & 4.97 & 92831 & stress \\
\hline 181 & B7SA66 & Hsp90-like protein & D. glomerata & 15 & 269 & 0.09 & 4.97 & 92918 & stress \\
\hline 182 & K0I7G7 & Thioredoxin peroxidase & N. tabacum & 9 & 55 & 0.09 & 8.03 & 29972 & redox \\
\hline 183 & K3Y4M8 & Uncharacterized protein & S. italica & 24 & 305 & 0.09 & 5.39 & 194893 & cell \\
\hline 184 & B6T6Q8 & Alcohol dehydrogenase class 3 & Z. mays & 4 & 155 & 0.09 & 7.02 & 41639 & misc \\
\hline 185 & F6H3T7 & Putative uncharacterized protein & V. vinifera & 16 & 153 & 0.09 & 6.11 & 40651 & not assigned \\
\hline 186 & K4A8Y9 & Uncharacterized protein & S. italica & 3 & 194 & 0.08 & 7.61 & 53531 & TCA \\
\hline 187 & Q5PY86 & NADH:cytochrome b5 reductase & V. fordii & 2 & 49 & 0.08 & 9.04 & 31341 & redox \\
\hline 188 & B6TLT1 & Serine/threonine-protein phosphatase $2 \mathrm{~A} 65 \mathrm{kDa}$ regulatory subunit A beta isoform & Z. mays & 16 & 93 & 0.08 & 5.03 & 66318 & protein \\
\hline 189 & Q0W9E2 & Beta chaperonin 60 & S. commersonii & 12 & 112 & 0.08 & 7.27 & 63333 & protein \\
\hline 190 & B4FH62 & NAD-dependent epimerase/dehydratase & Z. mays & 4 & 69 & 0.08 & 5.55 & 31917 & not assigned \\
\hline 191 & Q39834 & Clathrin heavy chain & G. $\max$ & 23 & 337 & 0.08 & 7.15 & 194430 & cell \\
\hline
\end{tabular}




\begin{tabular}{|c|c|c|c|c|c|c|c|c|c|}
\hline 192 & K3XVH0 & Uncharacterized protein & S. italica & 13 & 110 & 0.08 & 6.62 & 80203 & photosynthesis \\
\hline 193 & A8I1Q9 & Ser/thr protein kinase & C. reinhardtii & 3 & 68 & 0.08 & 7.14 & 33293 & protein \\
\hline 194 & I6LE48 & Uncharacterized protein & D. excelsa & 4 & 51 & 0.08 & 5.03 & 33659 & development \\
\hline 195 & F2DLJ7 & Predicted protein & H. vulgare & 10 & 190 & 0.08 & 7.77 & 44423 & hormone metabolism \\
\hline 196 & F2DWM5 & Predicted protein & H. vulgare & 13 & 134 & 0.08 & 9.22 & 92351 & development \\
\hline 197 & F2E2M4 & Predicted protein & H. vulgare & 16 & 156 & 0.08 & 5.29 & 92002 & stress \\
\hline 198 & Q8L994 & T-complex protein 1 subunit delta & A. thaliana & 3 & 69 & 0.08 & 7.68 & 58177 & protein \\
\hline 199 & E5RDE1 & $26 \mathrm{~S}$ proteasome non-ATPase regulatory subunit & C. melo & 4 & 53 & 0.08 & 4.73 & 34256 & protein \\
\hline 200 & I1LCM5 & Uncharacterized protein & G. $\max$ & 4 & 31 & 0.08 & 6.96 & 47008 & TCA \\
\hline 201 & Q8LAW8 & Serine/threonine-protein phosphatase & A. thaliana & 4 & 141 & 0.07 & 5.58 & 35539 & protein \\
\hline 202 & В6ТВ29 & Fructokinase-2 & Z. mays & 15 & 74 & 0.07 & 4.96 & 35875 & major $\mathrm{CHO}$ metabolism \\
\hline 203 & C8YNG6 & 3-ketoacyl CoA thiolase 1 & P. hybrida & 10 & 90 & 0.07 & 8.29 & 49405 & amino acid metabolism \\
\hline 204 & Q39986 & Cysteine proteinase & Hemerocallis sp. & 2 & 117 & 0.07 & 8.18 & 40006 & protein \\
\hline 205 & B9RI89 & Serine-threonine protein kinase, plant-type, putative & R. communis & 8 & 171 & 0.07 & 5.14 & 40375 & not assigned \\
\hline 206 & E1ZSN8 & Putative uncharacterized protein & C. variabilis & 5 & 55 & 0.07 & 5.50 & 39874 & cell \\
\hline 207 & E4MWY6 & Glucose-6-phosphate isomerase & T. halophila & 4 & 72 & 0.07 & 5.60 & 67312 & glycolysis \\
\hline 208 & F2DDZ5 & Predicted protein & H. vulgare & 2 & 71 & 0.06 & 6.00 & 40470 & TCA \\
\hline 209 & E4MXV1 & mRNA, clone: RTFL01-35-G02 & T. halophila & 2 & 40 & 0.06 & 5.48 & 40626 & protein \\
\hline 210 & K4A6E4 & Uncharacterized protein & S. italica & 10 & 68 & 0.06 & 5.16 & 83215 & protein \\
\hline 211 & B4FYM0 & Acetyl-CoA acetyltransferase, cytosolic 2 & Z. mays & 4 & 141 & 0.06 & 7.66 & 43450 & secondary metabolism \\
\hline 212 & K3XIP8 & Uncharacterized protein & S. italica & 4 & 50 & 0.06 & 6.40 & 43030 & protein \\
\hline 213 & Q6VWJ5 & Fructokinase 3 & S. lycopersicum & 3 & 101 & 0.06 & 5.80 & 41803 & major $\mathrm{CHO}$ metabolism \\
\hline 214 & B9HJ23 & Predicted protein & P. trichocarpa & 15 & 177 & 0.06 & 6.54 & 101700 & misc \\
\hline 215 & Q9M2T8 & 2-oxoglutarate dehydrogenase, E1 subunit-like protein & A. thaliana & 11 & 201 & 0.06 & 7.14 & 114888 & TCA \\
\hline 216 & O65879 & Translation initiation factor & P. sativum & 5 & 111 & 0.06 & 5.92 & 46184 & protein \\
\hline 217 & Q4ZJ73 & 12-oxophytodienoate reductase & H. brasiliensis & 5 & 34 & 0.06 & 7.46 & 44571 & hormone metabolism \\
\hline 218 & E5GBW6 & $26 \mathrm{~S}$ proteasome non-ATPase regulatory subunit & C. melo & 2 & 46 & 0.06 & 8.72 & 44456 & glycolysis \\
\hline 219 & Q8LGJ6 & Phosphoglycerate dehydrogenase-like protein & A. thaliana & 3 & 80 & 0.05 & 6.76 & 63556 & amino acid metabolism \\
\hline 220 & F2E797 & Predicted protein & H. vulgare & 5 & 89 & 0.05 & 8.43 & 47111 & TCA \\
\hline 221 & B6TVN4 & Phospholipase C & Z. mays & 10 & 161 & 0.05 & 5.71 & 46868 & signalling \\
\hline 222 & C5X277 & Putative uncharacterized protein $\mathrm{Sb} 02 \mathrm{~g} 026300$ & S. bicolor & 2 & 50 & 0.05 & 5.72 & 47330 & redox \\
\hline 223 & Q8H945 & Phosphoenolpyruvate carboxylase & L. japonicus & 16 & 107 & 0.05 & 5.88 & 111020 & glycolysis \\
\hline
\end{tabular}




\begin{tabular}{|c|c|c|c|c|c|c|c|c|c|}
\hline 224 & Q2LFC1 & AGO4-2 & N. benthamiana & 7 & 105 & 0.05 & 8.78 & 102331 & RNA \\
\hline 225 & K4A9K9 & Uncharacterized protein & S. italica & 2 & 34 & 0.05 & 7.65 & 52168 & secondary metabolism \\
\hline 226 & Q93YG1 & Monodehydroascorbate reductase & M. crystallinum & 7 & 114 & 0.05 & 5.66 & 51944 & redox \\
\hline 227 & C5WQZ5 & Putative uncharacterized protein Sb01g041210 & S. bicolor & 3 & 97 & 0.05 & 5.17 & 51802 & protein \\
\hline 228 & K3YPS9 & Uncharacterized protein & S. italica & 11 & 188 & 0.05 & 7.36 & 97789 & protein \\
\hline 229 & Q7XJH9 & Transaldolase & S. lycopersicum & 7 & 105 & 0.05 & 5.24 & 48062 & OPP \\
\hline 230 & I1LJS6 & Lipoxygenase & G. $\max$ & 14 & 304 & 0.05 & 5.83 & 104140 & hormone metabolism \\
\hline 231 & F2DDG9 & Predicted protein & H. vulgare & 4 & 86 & 0.05 & 7.33 & 52441 & cell \\
\hline 232 & F2E6F3 & Coatomer subunit gamma & H. vulgare & 4 & 156 & 0.05 & 5.96 & 100353 & cell \\
\hline 233 & Q43057 & Aspartate aminotransferase & P. miliaceum & 6 & 74 & 0.05 & 6.81 & 48154 & amino acid metabolism \\
\hline 234 & F2DIS4 & Malic enzyme & H. vulgare & 7 & 75 & 0.05 & 7.40 & 68411 & TCA \\
\hline 235 & Q9S768 & Alanine aminotransferase & O. sativa & 4 & 157 & 0.05 & 6.65 & 53130 & amino acid metabolism \\
\hline 236 & K3YRV8 & Uncharacterized protein & S. italica & 4 & 44 & 0.05 & 6.84 & 55802 & TCA \\
\hline 237 & Q38766 & Victorin binding protein & A. sativa & 8 & 90 & 0.05 & 8.21 & 112298 & photosynthesis \\
\hline 238 & E4MX61 & mRNA, clone: RTFL01-20-P01 & T. halophila & 6 & 72 & 0.05 & 6.60 & 53948 & glycolysis \\
\hline 239 & K3XWI6 & Uncharacterized protein & S. italica & 6 & 167 & 0.05 & 5.99 & 55489 & glycolysis \\
\hline 240 & F2D3S8 & Serine hydroxymethyltransferase & H. vulgare & 10 & 41 & 0.05 & 7.27 & 56215 & C1-metabolism \\
\hline 241 & K3YH94 & Uncharacterized protein & S. italica & 14 & 195 & 0.05 & 6.96 & 56064 & amino acid metabolism \\
\hline 242 & B5BLW2 & Carotenoid cleavage dioxygenase 1 & M. truncatula & 5 & 32 & 0.04 & 6.35 & 61063 & secondary metabolism \\
\hline 243 & E1Z4U0 & Putative uncharacterized protein & C. variabilis & 15 & 161 & 0.04 & 6.79 & 60152 & protein \\
\hline 244 & F2CTR2 & Predicted protein & H. vulgare & 8 & 110 & 0.04 & 6.76 & 59167 & protein \\
\hline 245 & F2DUH3 & Predicted protein & H. vulgare & 4 & 63 & 0.04 & 6.95 & 60964 & protein \\
\hline 246 & F2Z9R1 & Glucose-6-phosphate 1-dehydrogenase & N. benthamiana & 11 & 99 & 0.04 & 6.99 & 58894 & OPP \\
\hline 247 & K3ZS26 & Uncharacterized protein & S. italica & 4 & 33 & 0.04 & 5.63 & 60854 & mitoETC \\
\hline 248 & H9A1W2 & Acyl-activating enzyme 10 & C. sativa & 2 & 40 & 0.04 & 6.49 & 62013 & lipid metabolism \\
\hline 249 & B4FQM2 & Pyrophosphate--fructose 6-phosphate 1-phosphotransferase & Z. mays & 11 & 46 & 0.04 & 6.49 & 61607 & glycolysis \\
\hline 250 & B6T715 & Aldehyde dehydrogenase & Z. mays & 2 & 47 & 0.04 & 8.02 & 59108 & fermentation \\
\hline 251 & K3XFX7 & Uncharacterized protein & S. italica & 4 & 22 & 0.04 & 6.68 & 64026 & glycolysis \\
\hline 252 & K4A4Z8 & Uncharacterized protein & S. italica & 8 & 63 & 0.04 & 6.96 & 137642 & cell \\
\hline 253 & B2KNE6 & Phospholipase D & H. anпиия & 7 & 83 & 0.04 & 5.52 & 92315 & lipid metabolism \\
\hline 254 & Q4LDR0 & Heat shock protein & S. lycopersicum & 4 & 57 & 0.04 & 6.55 & 110564 & stress \\
\hline 255 & Q9LLB8 & Exoglucanase & Z. mays & 4 & 127 & 0.04 & 7.34 & 67356 & cell wall \\
\hline
\end{tabular}




\begin{tabular}{|c|c|c|c|c|c|c|c|c|c|}
\hline 256 & Q8L5C2 & $110 \mathrm{kDa} 4 \mathrm{SNc}-$ Tudor domain protein & P. sativum & 4 & 69 & 0.03 & 7.36 & 108600 & RNA \\
\hline 257 & A8IXU7 & Phototropin & C. reinhardtii & 12 & 74 & 0.03 & 8.47 & 81928 & signalling \\
\hline 258 & K3XVB0 & Uncharacterized protein & S. italica & 6 & 154 & 0.03 & 9.17 & 87653 & protein \\
\hline 259 & B6SXV4 & Peroxisomal fatty acid beta-oxidation multifunctional protein & Z. mays & 5 & 79 & 0.03 & 7.81 & 79104 & lipid metabolism \\
\hline 260 & K3YKV0 & Uncharacterized protein & S. italica & 12 & 101 & 0.03 & 5.54 & 87462 & cell \\
\hline 261 & E5GBL0 & Short-chain dehydrogenase/reductase & C. melo & 7 & 63 & 0.03 & 6.89 & 108844 & RNA \\
\hline 262 & Q9ZVY6 & $\mathrm{T} 25 \mathrm{~N} 20.17$ & A. thaliana & 2 & 50 & 0.03 & 8.09 & 90732 & protein \\
\hline 263 & A2JGX1 & Beta-galactosidase & S. lycopersicum & 3 & 38 & 0.03 & 5.29 & 98181 & misc \\
\hline 264 & Q9XFX7 & Phospholipase D & C. plantagineum & 6 & 36 & 0.03 & 7.74 & 92228 & lipid metabolism \\
\hline 265 & $\mathrm{~B} 1 \mathrm{P} 2 \mathrm{~T} 2$ & Cellulose synthase-like CslF3 & H. vulgare & 24 & 34 & 0.03 & 5.66 & 96260 & cell wall \\
\hline 266 & A8IM71 & Coatomer subunit gamma & C. reinhardtii & 4 & 37 & 0.03 & 5.58 & 97872 & cell \\
\hline 267 & F6HZ11 & Lipoxygenase & V. vinifera & 3 & 89 & 0.02 & 6.04 & 99463 & hormone metabolism \\
\hline 268 & J3MFV3 & Lipoxygenase & O. brachyantha & 7 & 54 & 0.02 & 6.77 & 103742 & hormone metabolism \\
\hline 269 & Q43248 & Acetyl CoA carboxylase & Z. mays & 8 & 37 & 0.02 & 6.28 & 188959 & lipid metabolism \\
\hline 270 & Q42793 & Acetyl CoA carboxylase & G. $\max$ & 7 & 63 & 0.01 & 6.32 & 253225 & lipid metabolism \\
\hline 271 & E4MXV2 & mRNA, clone: RTFL01-41-F15 & T. halophila & 7 & 67 & - & 10.49 & 64768 & secondary metabolism \\
\hline 272 & B3TM07 & Ribosomal protein L17 & E. guineensis & 10 & 66 & - & 6.09 & 20744 & protein \\
\hline
\end{tabular}

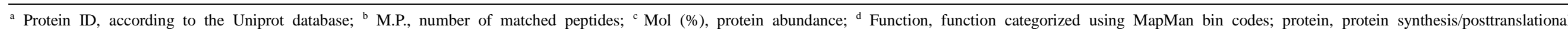

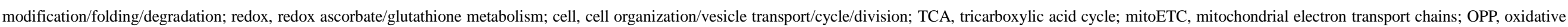
pentose phosphate; DNA, DNA synthesis/repair; RNA, RNA processing/regulation of transcription; and misc, miscellaneous. 
Supplemental Table 6. Proteins Identified in L. japonica Immature Flower Buds at Fraction of Flow Through of pH 9 using Combinatorial Peptide Ligand Libraries Fractionation.

\begin{tabular}{|c|c|c|c|c|c|c|c|c|c|}
\hline No. & Protein ID ${ }^{\text {a }}$ & Description & Species & M.P. ${ }^{\mathrm{b}}$ & Score & $\operatorname{Mol}(\%)^{\mathrm{c}}$ & $\mathrm{pI}$ & Mass (Da) & Function $^{\mathrm{d}}$ \\
\hline 1 & C4B8E5 & Glyceraldehyde-3-phosphate dehydrogenase & T. gesneriana & 122 & 3774 & 1.38 & 7.96 & 20964 & glycolysis \\
\hline 2 & Q9M6B3 & Malate dehydrogenase & V. vinifera & 115 & 2958 & 1.15 & 8.62 & 37137 & TCA \\
\hline 3 & Q5GLI7 & Ribulose 1,5-bisphosphate carboxylase/oxygenase large subunit & E. ghellinckii & 153 & 1484 & 0.95 & 6.89 & 48283 & photosynthesis \\
\hline 4 & B3TLP4 & Ribosomal protein L30 & E. guineensis & 7 & 68 & 0.94 & 9.72 & 12451 & protein \\
\hline 5 & Q41534 & ATP synthase subunit B. & T. aestivum & 93 & 2260 & 0.92 & 5.86 & 59326 & mitoETC \\
\hline 6 & Q6PKU2 & Putative glyceraldehyde-3-phosphate dehydrogenase & O. minor & 87 & 2395 & 0.87 & 7.09 & 22122 & glycolysis \\
\hline 7 & H1ZY49 & Translation elongation factor 1-alpha & L. maackii & 22 & 195 & 0.86 & 5.00 & 11619 & protein \\
\hline 8 & A8MQP1 & Adenosylhomocysteinase 1 & A. thaliana & 45 & 643 & 0.85 & 5.16 & 36022 & amino acid metabolism \\
\hline 9 & F2D884 & Predicted protein & H. vulgare & 102 & 1755 & 0.84 & 5.19 & 71476 & stress \\
\hline 10 & $\mathrm{O} 03843$ & Ribulose-bisphosphate carboxylase & T. glandulifera & 150 & 1940 & 0.80 & 6.04 & 53346 & photosynthesis \\
\hline 11 & C6GFP3 & ATP synthase subunit B. & G. hirsutum & 91 & 2315 & 0.80 & 6.29 & 59987 & mitoETC \\
\hline 12 & D6C638 & Ribulose-1,5-bisphosphate carboxylase/oxygenase large subunit & P. sp. PSN 1 & 91 & 904 & 0.76 & 6.58 & 50770 & photosynthesis \\
\hline 13 & K4ACE3 & Uncharacterized protein & S. italica & 100 & 1960 & 0.70 & 6.09 & 35803 & TCA \\
\hline 14 & Q645N1 & Malate dehydrogenase & S. lycopersicum & 47 & 1378 & 0.68 & 8.72 & 36357 & TCA \\
\hline 15 & Q5KTY2 & Ribulose-1,5-bisphosphate carboxylase large subunit & A. sutepensis & 118 & 1093 & 0.67 & 6.92 & 51143 & photosynthesis \\
\hline 16 & Q08II7 & Heat shock protein 70-like protein & L. sativa & 9 & 381 & 0.65 & 10.33 & 12539 & stress \\
\hline 17 & J3RTS9 & Glyceraldehyde-3-phosphate dehydrogenase & C. pentagona & 140 & 3564 & 0.65 & 7.23 & 32382 & glycolysis \\
\hline 18 & I6QZA8 & Malate dehydrogenase & E.m coca & 84 & 1936 & 0.60 & 7.62 & 39915 & TCA \\
\hline 19 & F2D6I8 & Predicted protein & H. vulgare & 69 & 2095 & 0.59 & 7.14 & 36874 & glycolysis \\
\hline 20 & Q9TKG9 & Ribulose 1,5-bisphosphate carboxylase large subunit & R. tomentosum & 139 & 1244 & 0.59 & 7.11 & 52772 & photosynthesis \\
\hline 21 & Q9FEM4 & Catalase & B. pendula & 28 & 320 & 0.58 & 5.48 & 17247 & redox \\
\hline 22 & Q9TKH9 & Ribulose 1,5-bisphosphate carboxylase large subunit & H. albomarginata & 102 & 1237 & 0.57 & 6.37 & 51833 & photosynthesis \\
\hline 23 & K3XJN7 & Uncharacterized protein & S. italica & 48 & 878 & 0.56 & 7.85 & 35629 & TCA \\
\hline 24 & Q4H1G1 & Adenosylhomocysteinase & B. vulgaris & 54 & 675 & 0.56 & 6.19 & 54052 & amino acid metabolism \\
\hline 25 & D9ZBV9 & Malate dehydrogenase & S. globosa & 18 & 369 & 0.56 & 4.92 & 13766 & TCA \\
\hline 26 & B4FNW1 & Triosephosphate isomerase & Z. mays & 18 & 211 & 0.52 & 5.68 & 27236 & glycolysis \\
\hline 27 & Q6B443 & $26 \mathrm{~S}$ proteasome B. subunit & L. minor & 11 & 248 & 0.51 & 9.72 & 12530 & protein \\
\hline 28 & Q84RD8 & Adenosylhomocysteinase & M. truncatula & 47 & 685 & 0.48 & 6.01 & 53790 & amino acid metabolism \\
\hline 29 & Q7DM38 & Ribulose bisphosphate carboxylase small chain & P. vulgaris & 39 & 183 & 0.48 & 8.43 & 15877 & photosynthesis \\
\hline 30 & K3Z913 & Uncharacterized protein & S. italica & 28 & 543 & 0.48 & 9.47 & 26793 & cell \\
\hline 31 & E5GBV8 & Glyceraldehyde-3-phosphate dehydrogenase & C. melo & 63 & 1591 & 0.47 & 7.12 & 36754 & glycolysis \\
\hline
\end{tabular}




\begin{tabular}{|c|c|c|c|c|c|c|c|c|c|}
\hline 32 & D7NHW9 & 2-phospho-D-glycerate hydrolase & P. trifoliata & 73 & 1972 & 0.47 & 5.78 & 47986 & glycolysis \\
\hline 33 & E0D6S1 & Glyceraldehyde 3-phosphate dehydrogenase & G. bicolor & 62 & 1771 & 0.46 & 6.13 & 36957 & glycolysis \\
\hline 34 & D6PPS8 & AT3G02360-like protein & C. grandiflora & 9 & 161 & 0.45 & 9.22 & 21010 & OPP \\
\hline 35 & Q9SAT7 & Superoxide dismutase $[\mathrm{Cu}-\mathrm{Zn}]$ & P. tremuloides & 11 & 114 & 0.42 & 5.82 & 15420 & redox \\
\hline 36 & A4ZSZ4 & Actin & P. trichocarpa & 23 & 595 & 0.42 & 5.49 & 41913 & cell \\
\hline 37 & Q6T379 & Triosephosphate isomerase & S. chacoense & 17 & 275 & 0.41 & 5.99 & 27251 & glycolysis \\
\hline 38 & F2CQQ3 & Predicted protein & H. vulgare & 32 & 909 & 0.40 & 5.16 & 54310 & transport \\
\hline 39 & B6SJZ8 & Glyceraldehyde-3-phosphate dehydrogenase, cytosolic & Z. mays & 74 & 1821 & 0.39 & 6.96 & 36614 & glycolysis \\
\hline 40 & B6TCZ3 & Malate dehydrogenase & Z. mays & 47 & 676 & 0.39 & 7.74 & 41394 & TCA \\
\hline 41 & Q9SW73 & Isocitrate dehydrogenase [NADP] & C. limon & 19 & 281 & 0.39 & 6.95 & 46823 & TCA \\
\hline 42 & $\mathrm{~F} 2 \mathrm{E} 4 \mathrm{C} 2$ & Predicted protein & H. vulgare & 69 & 1062 & 0.38 & 5.25 & 72202 & stress \\
\hline 43 & G0WP59 & Superoxide dismutase $[\mathrm{Cu}-\mathrm{Zn}]$ & W. somnifera & 24 & 529 & 0.38 & 5.64 & 15782 & redox \\
\hline 44 & Q40463 & NTGB2 & N.tabacum & 6 & 183 & 0.38 & 6.19 & 15827 & signalling \\
\hline 45 & B2VQE0 & Methionine synthase & O. ramosa & 113 & 1733 & 0.37 & 6.40 & 84862 & amino acid metabolism \\
\hline 46 & K3YQW5 & Uncharacterized protein & S. italica & 31 & 587 & 0.37 & 5.39 & 68637 & transport \\
\hline 47 & B3TLL5 & 40S ribosomal protein S19 & E. guineensis & 21 & 99 & 0.37 & 10.04 & 16202 & protein \\
\hline 48 & Q9SN95 & AT3G46440 protein & A. thaliana & 20 & 344 & 0.37 & 7.52 & 38593 & cell wall \\
\hline 49 & B3TLL4 & Triosephosphate isomerase & E. guineensis & 17 & 159 & 0.36 & 6.23 & 27476 & glycolysis \\
\hline 50 & O81830 & Putative uncharacterized protein AT4g27270 & A. thaliana & 6 & 174 & 0.36 & 6.79 & 22355 & lipid metabolism \\
\hline 51 & A8DSD0 & Alpha-tubulin 7 & P. tremuloides & 23 & 337 & 0.36 & 5.15 & 50382 & cell \\
\hline 52 & K3ZUR5 & Uncharacterized protein & S. italica & 16 & 545 & 0.35 & 6.24 & 36749 & co-factor and vitamine metabolism \\
\hline 53 & Q5ZF83 & Putative uncharacterized protein & P. major & 19 & 185 & 0.35 & 7.28 & 25390 & cell wall \\
\hline 54 & E4MXI2 & mRNA, clone: RTFL01-39-D20 & T. halophila & 44 & 680 & 0.34 & 5.21 & 73890 & stress \\
\hline 55 & B3TLQ5 & $60 \mathrm{~S}$ ribosomal protein $\mathrm{L} 11$ & E. guineensis & 10 & 241 & 0.33 & 9.92 & 20950 & protein \\
\hline 56 & K3XFR6 & Uncharacterized protein & S. italica & 25 & 462 & 0.33 & 7.46 & 66448 & TCA \\
\hline 57 & Q8LC80 & Putative calcium-binding protein, calreticulin & A. thaliana & 52 & 887 & 0.33 & 4.53 & 48385 & signalling \\
\hline 58 & B6SHD3 & Malate dehydrogenase & Z. mays & 46 & 584 & 0.32 & 8.32 & 42623 & TCA \\
\hline 59 & Q19TV8 & UDP-glucose pyrophosphorylase & C. melo & 23 & 305 & 0.32 & 7.17 & 52201 & glycolysis \\
\hline 60 & D0ELH5 & Peptidyl-prolyl cis-trans isomerase & C. sinensis & 17 & 328 & 0.31 & 8.46 & 18310 & cell \\
\hline 61 & H6VP90 & ATP synthase subunit B. & C. lobatus & 19 & 387 & 0.30 & 5.19 & 47813 & photosynthesis \\
\hline 62 & G5DVX2 & Phosphoglycerate kinase & S. latifolia & 45 & 913 & 0.30 & 6.58 & 51352 & photosynthesis \\
\hline 63 & B6EBD6 & Heat shock protein $90-2$ & G. $\max$ & 46 & 606 & 0.30 & 5.07 & 80392 & stress \\
\hline
\end{tabular}




\begin{tabular}{|c|c|c|c|c|c|c|c|c|c|}
\hline 64 & Q9ATF4 & Ribosomal protein L33 & C. sativa & 6 & 216 & 0.30 & 10.58 & 12831 & protein \\
\hline 65 & A2V880 & G protein B.-subunit-like protein & N.tabacum & 4 & 50 & 0.30 & 7.09 & 16100 & signalling \\
\hline 66 & Q1KUX5 & 6-phosphogluconate dehydrogenase, decarboxylating & C. spinosa & 27 & 224 & 0.29 & 6.06 & 53777 & OPP \\
\hline 67 & Q5IA96 & Cytochrome oxidase subunit II & A. trichopoda & 2 & 87 & 0.28 & 4.75 & 9794 & mitoETC \\
\hline 68 & D0EJY9 & Molecular chaperone Hsp90-3 & N.benthamiana & 38 & 466 & 0.27 & 5.02 & 80638 & stress \\
\hline 69 & K3XEI1 & Uncharacterized protein & S. italica & 52 & 720 & 0.27 & 6.27 & 94876 & protein \\
\hline 70 & Q06Z74 & Fibrillin 4 & C. canephora & 11 & 260 & 0.26 & 8.88 & 32064 & cell \\
\hline 71 & H9NIE1 & Cell division cycle protein 48 & C. sinensis & 44 & 1090 & 0.26 & 5.29 & 90644 & cell \\
\hline 72 & $\mathrm{D} 2 \mathrm{X} 5 \mathrm{~K} 2$ & DEAD-box RNA helicase-like protein & P. persica & 27 & 772 & 0.26 & 5.69 & 47191 & protein \\
\hline 73 & F2EL23 & Predicted protein & H. vulgare & 37 & 937 & 0.26 & 5.29 & 90854 & cell \\
\hline 74 & B6TN41 & Regulator of ribonuclease activity A & Z. mays & 2 & 30 & 0.26 & 6.18 & 18390 & C1-metabolism \\
\hline 75 & J9QAL9 & Ribosomal protein L2 & A. corniculatum & 5 & 128 & 0.25 & 10.23 & 21703 & protein \\
\hline 76 & Q9AXR6 & ATP:citrate lyase & C. аппиит & 28 & 625 & 0.25 & 7.39 & 66376 & TCA \\
\hline 77 & D6PAY2 & Peptidyl-prolyl cis-trans isomerase & V. hybrid cultivar & 27 & 313 & 0.25 & 8.46 & 18582 & cell \\
\hline 78 & F2DAU4 & Predicted protein & H. vulgare & 33 & 464 & 0.25 & 7.56 & 62696 & protein \\
\hline 79 & $\mathrm{O} 23254$ & Serine hydroxymethyltransferase & A. thaliana & 50 & 863 & 0.25 & 7.23 & 52141 & C1-metabolism \\
\hline 80 & Q7XTK8 & Catalase & $P$. persica & 39 & 658 & 0.25 & 7.43 & 57289 & redox \\
\hline 81 & O81609 & Nodule-enhanced malate dehydrogenase & P. sativum & 15 & 486 & 0.24 & 7.75 & 42106 & TCA \\
\hline 82 & Q8GZD5 & Xyloglucan endotransglycosylase & P. tremula & 29 & 400 & 0.24 & 10.20 & 34481 & cell wall \\
\hline 83 & Q6H8J2 & $40 \mathrm{~S}$ ribosomal protein $\mathrm{S} 9$ & C. roseus & 13 & 65 & 0.24 & 7.75 & 23015 & protein \\
\hline 84 & B6TJ90 & Histone H4 & Z. mays & 9 & 119 & 0.23 & 9.67 & 20120 & cell \\
\hline 85 & A4UMS1 & Myo-inositol-1-phosphate synthase isoform 1 & X. viscosa & 24 & 582 & 0.23 & 5.41 & 56899 & minor $\mathrm{CHO}$ metabolism \\
\hline 86 & B5LAU5 & DH putative B.-hydroxyacyl-ACP dehydratase & C. аппиит & 7 & 42 & 0.23 & 9.29 & 24080 & lipid metabolism \\
\hline 87 & Q8MCY2 & Ribulose bisphosphate carboxylase large chain & V. baccifera & 45 & 415 & 0.23 & 6.64 & 48739 & photosynthesis \\
\hline 88 & A1BQW9 & Transketolase & N.attenuata & 24 & 92 & 0.23 & 6.80 & 32653 & OPP \\
\hline 89 & F2CQ27 & Predicted protein & H. vulgare & 8 & 49 & 0.22 & 6.66 & 25460 & signalling \\
\hline 90 & K3Z5U9 & Uncharacterized protein & S. italica & 36 & 584 & 0.22 & 6.47 & 49826 & photosynthesis \\
\hline 91 & A2Q5W0 & Alpha-tubulin & M. truncatula & 18 & 460 & 0.22 & 5.10 & 50457 & cell \\
\hline 92 & K3YSB2 & Uncharacterized protein & S. italica & 21 & 133 & 0.22 & 8.63 & 50388 & amino acid metabolism \\
\hline 93 & Q9SRV5 & 5-methyltetrahydropteroyltriglutamate--homocysteine methyltransferase & A. thaliana & 73 & 1170 & 0.21 & 6.62 & 84873 & amino acid metabolism \\
\hline 94 & Q069K2 & 5-methyltetrahydropteroyltriglutamate--homocysteine methyltransferase & N.suaveolens & 54 & 883 & 0.21 & 6.51 & 85060 & amino acid metabolism \\
\hline 95 & Q8RVT3 & Elongation factor EF-2 & P. sativum & 35 & 340 & 0.21 & 6.18 & 55623 & protein \\
\hline
\end{tabular}




\begin{tabular}{|c|c|c|c|c|c|c|c|c|c|}
\hline 96 & $\mathrm{O} 48903$ & Malate dehydrogenase & M. sativa & 19 & 431 & 0.21 & 8.29 & 38397 & gluconeogenesis \\
\hline 97 & G5DXP1 & Fructose-bisphosphate aldolase & S. latifolia & 11 & 464 & 0.21 & 6.16 & 42771 & photosynthesis \\
\hline 98 & Q9SXX4 & Fructose-bisphosphate aldolase & N.paniculata & 10 & 327 & 0.21 & 7.33 & 42832 & photosynthesis \\
\hline 99 & G5DXR5 & UDP-glucuronic acid decarboxylase & S. latifolia & 15 & 323 & 0.21 & 7.27 & 38756 & cell wall \\
\hline 100 & F2D690 & Predicted protein & H. vulgare & 4 & 129 & 0.21 & 4.37 & 12678 & protein \\
\hline 101 & A3QQY2 & Sucrose synthase & C. intybus & 41 & 363 & 0.21 & 5.53 & 92338 & major $\mathrm{CHO}$ metabolism \\
\hline 102 & K3YVE3 & Uncharacterized protein & S. italica & 8 & 213 & 0.21 & 6.39 & 25864 & protein \\
\hline 103 & A1BLP6 & Thioredoxin & M. truncatula & 11 & 33 & 0.21 & 6.16 & 12904 & redox \\
\hline 104 & $\mathrm{~A} 2 \mathrm{PZC} 2$ & UDP-Glucose:protein transglucosylase & C. reinhardtii & 7 & 111 & 0.20 & 6.33 & 39846 & cell wall \\
\hline 105 & B6TGL7 & Enolase & Z. mays & 12 & 467 & 0.20 & 6.23 & 50512 & glycolysis \\
\hline 106 & H2BBB1 & ATP synthase subunit alpha, chloroplastic & P. diguetii & 12 & 125 & 0.20 & 5.74 & 55710 & photosynthesis \\
\hline 107 & В3H4P2 & Glyceraldehyde-3-phosphate dehydrogenase (NADP+) & A. thaliana & 6 & 64 & 0.19 & 7.77 & 37929 & photosynthesis \\
\hline 108 & $\mathrm{~K} 3 \mathrm{XXC5}$ & Uncharacterized protein & S. italica & 52 & 558 & 0.19 & 6.68 & 42409 & glycolysis \\
\hline 109 & A9ZMZ5 & Acetyl-CoA C-acetyltransferase & H. brasiliensis & 29 & 978 & 0.19 & 7.06 & 42039 & secondary metabolism \\
\hline 110 & Q9SH69 & 6-phosphogluconate dehydrogenase, decarboxylating & A. thaliana & 12 & 289 & 0.18 & 5.45 & 53686 & OPP \\
\hline 111 & E4MVY3 & mRNA, clone: RTFL01-01-M18 & T. halophila & 24 & 491 & 0.18 & 6.25 & 94734 & protein \\
\hline 112 & D3GQL1 & Aconitate hydratase 3 & C. clementina & 60 & 957 & 0.18 & 6.30 & 98669 & TCA \\
\hline 113 & B6TPE4 & Glutamate-1-semialdehyde 2,1-aminomutase & Z. mays & 8 & 62 & 0.18 & 6.55 & 50223 & tetrapyrrole synthesis \\
\hline 114 & K3YG24 & Uncharacterized protein & S. italica & 40 & 523 & 0.18 & 7.31 & 107135 & TCA \\
\hline 115 & B4FPK8 & Cytochrome b5 & Z. mays & 4 & 84 & 0.18 & 5.30 & 14958 & redox \\
\hline 116 & A8MQR4 & $60 \mathrm{~S}$ acidic ribosomal protein $\mathrm{P} 0-2$ & A. thaliana & 11 & 160 & 0.17 & 4.84 & 30656 & protein \\
\hline 117 & F2D448 & Predicted protein & H. vulgare & 24 & 330 & 0.17 & 10.17 & 30607 & protein \\
\hline 118 & B6T6Q8 & Alcohol dehydrogenase class 3 & Z. mays & 11 & 322 & 0.17 & 7.02 & 41639 & misc \\
\hline 119 & K3XVH0 & Uncharacterized protein & S. italica & 43 & 200 & 0.17 & 6.62 & 80203 & photosynthesis \\
\hline 120 & Q3KN68 & Isoflavone reductase-like protein 5 & V. vinifera & 15 & 82 & 0.17 & 6.10 & 33865 & secondary metabolism \\
\hline 121 & Q645M9 & Glyoxisomal malate dehydrogenase & S. lycopersicum & 15 & 365 & 0.17 & 7.94 & 38007 & gluconeogenesis \\
\hline 122 & K3YR95 & Uncharacterized protein & S. italica & 20 & 181 & 0.16 & 7.65 & 58776 & protein \\
\hline 123 & Q8LG68 & UDP-glucose 6-dehydrogenase & A. thaliana & 16 & 206 & 0.16 & 6.18 & 53591 & stress \\
\hline 124 & E5GBS0 & $40 \mathrm{~S}$ ribosomal protein $\mathrm{S} 24$ & C. melo & 7 & 157 & 0.16 & 10.64 & 15761 & protein \\
\hline 125 & F2D861 & Predicted protein & H. vulgare & 8 & 384 & 0.16 & 10.43 & 15765 & protein \\
\hline 126 & K3YZ28 & Uncharacterized protein & S. italica & 4 & 154 & 0.16 & 5.49 & 15971 & protein \\
\hline 127 & F2CQP8 & Predicted protein & H. vulgare & 7 & 88 & 0.16 & 10.59 & 32811 & biodegradation of xenobiotics. \\
\hline
\end{tabular}




\begin{tabular}{|c|c|c|c|c|c|c|c|c|c|}
\hline 128 & O04428 & Putative uncharacterized protein & C. paradisi & 13 & 212 & 0.16 & 5.59 & 32737 & biodegradation of xenobiotics. \\
\hline 129 & D7UPN0 & SEC13 family protein & L. japonicus & 5 & 60 & 0.16 & 6.01 & 32940 & protein \\
\hline 130 & F2EE28 & Predicted protein & H. vulgare & 12 & 192 & 0.16 & 5.55 & 61280 & protein \\
\hline 131 & K4A9V6 & Uncharacterized protein & S. italica & 17 & 193 & 0.16 & 5.10 & 50519 & cell \\
\hline 132 & Q84QC6 & Ubiquitin-conjugating enzyme & H. vulgare & 6 & 26 & 0.16 & 7.02 & 16667 & protein \\
\hline 133 & $\mathrm{~F} 2 \mathrm{CYN} 2$ & Predicted protein & H. vulgare & 52 & 794 & 0.15 & 6.18 & 84718 & amino acid metabolism \\
\hline 134 & A5JVC6 & Putative uncharacterized protein & B. campestris & 4 & 79 & 0.15 & 6.25 & 34259 & cell wall \\
\hline 135 & K3XZA2 & Uncharacterized protein & S. italica & 19 & 235 & 0.15 & 9.58 & 23221 & cell \\
\hline 136 & F2DBE0 & Predicted protein & H. vulgare & 6 & 116 & 0.15 & 8.22 & 34595 & TCA \\
\hline 137 & K3ZSX0 & Uncharacterized protein & S. italica & 8 & 239 & 0.15 & 7.64 & 53564 & amino acid metabolism \\
\hline 138 & K3Z566 & Uncharacterized protein & S. italica & 29 & 401 & 0.15 & 7.77 & 59005 & C1-metabolism \\
\hline 139 & Q7XAE2 & Putative fructokinase 2 & P. integrifolia & 23 & 220 & 0.15 & 5.35 & 35181 & major $\mathrm{CHO}$ metabolism \\
\hline 140 & Q6VWJ5 & Fructokinase 3 & S. lycopersicum & 7 & 142 & 0.15 & 5.80 & 41803 & major $\mathrm{CHO}$ metabolism \\
\hline 141 & Q4H1G2 & Methionine synthase & B. vulgaris & 65 & 1342 & 0.15 & 6.46 & 88546 & amino acid metabolism \\
\hline 142 & E4MXI1 & mRNA, clone: RTFL01-39-B07 & T. halophila & 10 & 270 & 0.15 & 6.98 & 48063 & photosynthesis \\
\hline 143 & E5F725 & Putative uncharacterized protein & E. parvulum & 5 & 201 & 0.14 & 7.11 & 42041 & cell wall \\
\hline 144 & В3ТM23 & $40 \mathrm{~S}$ ribosomal protein $\mathrm{S} 4$ & E. guineensis & 27 & 263 & 0.14 & 10.24 & 30023 & protein \\
\hline 145 & A0EJL8 & GDP-D-mannose-3',5'-epimerase & M. glabra & 23 & 157 & 0.14 & 6.21 & 42927 & redox \\
\hline 146 & Q9SP13 & Nucleoside diphosphate kinase & P. sativum & 8 & 47 & 0.13 & 9.32 & 25362 & not assigned \\
\hline 147 & K3XGJ6 & Uncharacterized protein & S. italica & 11 & 103 & 0.13 & 5.22 & 58129 & protein \\
\hline 148 & B7UCR9 & 1-deoxy-d-xylulose 5-phosphate reductoisomerase & S. miltiorrhiza & 12 & 152 & 0.13 & 6.40 & 51960 & secondary metabolism \\
\hline 149 & K3ZA95 & Uncharacterized protein & S. italica & 26 & 35 & 0.13 & 7.33 & 19471 & photosynthesis \\
\hline 150 & F2DM28 & Predicted protein & H. vulgare & 4 & 121 & 0.13 & 8.53 & 38865 & biodegradation of xenobiotics. \\
\hline 151 & Q8L8H6 & Phytoene desaturase & T. erecta & 2 & 44 & 0.13 & 6.70 & 38216 & secondary metabolism \\
\hline 152 & C1IC54 & Cinnamyl alcohol dehydrogenase & G. hirsutum & 9 & 79 & 0.13 & 6.25 & 39574 & secondary metabolism \\
\hline 153 & $\mathrm{C} 1 \mathrm{M} 2 \mathrm{~W} 0$ & Somatic embryogenesis cinnamyl alcohol dehydrogenase 1 & C. sativus & 17 & 297 & 0.13 & 7.44 & 39462 & secondary metabolism \\
\hline 154 & E4MX73 & mRNA, clone: RTFL01-10-N01 & T. halophila & 10 & 249 & 0.13 & 5.83 & 39684 & TCA \\
\hline 155 & E4MWY6 & Glucose-6-phosphate isomerase & T. halophila & 7 & 191 & 0.13 & 5.60 & 67312 & glycolysis \\
\hline 156 & Q7XJH9 & Transaldolase & S. lycopersicum & 7 & 105 & 0.13 & 5.96 & 48062 & OPP \\
\hline 157 & D2XNF3 & Chaperone protein DnaK & P. patens & 12 & 77 & 0.13 & 5.50 & 75674 & stress \\
\hline 158 & F6H3T7 & Putative uncharacterized protein & $V$. vinifera & 19 & 158 & 0.12 & 8.09 & 40651 & not assigned \\
\hline 159 & D8L9Q5 & Fructose-bisphosphate aldolase & T. aestivum & 12 & 294 & 0.12 & 6.11 & 41564 & photosynthesis \\
\hline
\end{tabular}




\begin{tabular}{|c|c|c|c|c|c|c|c|c|c|}
\hline 160 & F2D3S8 & Serine hydroxymethyltransferase & H. vulgare & 29 & 228 & 0.12 & 8.21 & 56215 & C1-metabolism \\
\hline 161 & K3YR06 & Uncharacterized protein & S. italica & 12 & 499 & 0.12 & 6.81 & 64225 & protein \\
\hline 162 & K3XFY9 & Uncharacterized protein & S. italica & 19 & 218 & 0.12 & 6.16 & 64010 & cell \\
\hline 163 & F2DWX8 & Predicted protein & H. vulgare & 2 & 27 & 0.12 & 6.39 & 21692 & lipid metabolism \\
\hline 164 & K3ZW60 & Uncharacterized protein & S. italica & 7 & 65 & 0.11 & 9.76 & 29913 & protein \\
\hline 165 & K4A7I4 & Uncharacterized protein & S. italica & 7 & 230 & 0.11 & 5.40 & 61666 & photosynthesis \\
\hline 166 & Q0MYQ7 & Germin-like protein 2 & V. vinifera & 4 & 229 & 0.11 & 8.41 & 22832 & stress \\
\hline 167 & Q1EMR1 & Nucleoside-diphopshate-sugar dehydratase & P. major & 7 & 69 & 0.11 & 5.17 & 22710 & cell wall \\
\hline 168 & Q9STA4 & Cysteine protease & M. sativa & 2 & 27 & 0.11 & 5.57 & 22875 & protein \\
\hline 169 & K3XZE3 & Uncharacterized protein & S. italica & 4 & 160 & 0.11 & 5.27 & 23153 & protein \\
\hline 170 & O78327 & Transketolase 1 & C. аппиит & 33 & 174 & 0.11 & 6.62 & 80398 & OPP \\
\hline 171 & A4UTS5 & Chloroplast ferredoxin-NADP+ reductase & P. sativum & 11 & 225 & 0.11 & 8.40 & 40392 & photosynthesis \\
\hline 172 & J7MPY1 & ATP synthase subunit alpha & M. domestica & 15 & 37 & 0.11 & 6.61 & 55600 & mitoETC \\
\hline 173 & B6TGB2 & Elongation factor 1-gamma 3 & Z. mays & 11 & 75 & 0.11 & 6.37 & 47357 & protein \\
\hline 174 & A8MRE8 & Triosephosphate isomerase & A. thaliana & 5 & 133 & 0.11 & 7.49 & 32458 & photosynthesis \\
\hline 175 & B4FH62 & NAD-dependent epimerase/dehydratase & Z. mays & 8 & 194 & 0.11 & 9.04 & 31917 & not assigned \\
\hline 176 & A8JHR9 & Glyceraldehyde 3-phosphate dehydrogenase, dominant splicing variant & C. reinhardtii & 6 & 157 & 0.11 & 8.95 & 39963 & photosynthesis \\
\hline 177 & B5LAU9 & Glutamine synthetase & C. аппиит & 17 & 212 & 0.10 & 11.00 & 47926 & N-metabolism \\
\hline 178 & E5LCX1 & C14 cysteine protease & S. demissum & 5 & 145 & 0.10 & 6.93 & 23974 & protein \\
\hline 179 & $\mathrm{~K} 3 \mathrm{XX} 30$ & Uncharacterized protein & S. italica & 7 & 93 & 0.10 & 5.90 & 47991 & protein \\
\hline 180 & A8MQA1 & $60 \mathrm{~S}$ ribosomal protein $\mathrm{L} 13$ & A. thaliana & 3 & 114 & 0.10 & 4.98 & 23525 & protein \\
\hline 181 & E4MXR5 & mRNA, clone: RTFL01-40-M04 & T. halophila & 21 & 283 & 0.10 & 5.07 & 73489 & stress \\
\hline 182 & B3TLP3 & Translation elongation factor EF-1 B. chain & E. guineensis & 8 & 209 & 0.10 & 4.69 & 24523 & protein \\
\hline 183 & B3TLY3 & Ribosomal protein L15 & E. guineensis & 2 & 64 & 0.10 & 11.49 & 24371 & protein \\
\hline 184 & K3ZU91 & Uncharacterized protein & S. italica & 14 & 107 & 0.10 & 8.70 & 42567 & protein \\
\hline 185 & A9PAK9 & Putative uncharacterized protein & P. trichocarpa & 7 & 187 & 0.10 & 6.89 & 50623 & amino acid metabolism \\
\hline 186 & H6VUT7 & Translation elongation factor & A. mongolicus & 4 & 104 & 0.10 & 4.63 & 25300 & protein \\
\hline 187 & F2DP49 & Predicted protein & H. vulgare & 6 & 109 & 0.10 & 8.63 & 25913 & hormone metabolism \\
\hline 188 & K4A8Y9 & Uncharacterized protein & S. italica & 6 & 205 & 0.10 & 5.63 & 53531 & TCA \\
\hline 189 & G8HAB2 & PLP-dependent aminotransferase & P. somniferum & 9 & 199 & 0.10 & 9.16 & 53501 & amino acid metabolism \\
\hline 190 & K3ZD99 & Uncharacterized protein & S. italica & 3 & 49 & 0.10 & 7.61 & 26325 & transport \\
\hline 191 & H9C956 & 3-deoxy-D-arabino-heptulosonate 7-phosphate synthase & N.benthamiana & 14 & 132 & 0.10 & 8.28 & 53191 & amino acid metabolism \\
\hline
\end{tabular}




\begin{tabular}{|c|c|c|c|c|c|c|c|c|c|}
\hline 192 & E4MWP5 & mRNA, clone: RTFL01-15-G22 & T. halophila & 17 & 64 & 0.09 & 6.16 & 35258 & photosynthesis \\
\hline 193 & E3WHD5 & Lipoxygenase & V. hybrid cultivar & 15 & 279 & 0.09 & 6.55 & 100738 & hormone metabolism \\
\hline 194 & F2E2M4 & Predicted protein & H. vulgare & 13 & 253 & 0.09 & 5.29 & 92002 & stress \\
\hline 195 & F2CW31 & Predicted protein & H. vulgare & 10 & 175 & 0.09 & 6.21 & 63075 & glycolysis \\
\hline 196 & С0Р9J6 & Aminoaldehyde dehydrogenase 1 & Z. mays & 5 & 109 & 0.09 & 5.45 & 55733 & secondary metabolism \\
\hline 197 & F2D5T2 & Predicted protein & H. vulgare & 3 & 110 & 0.09 & 8.09 & 27859 & mitoETC \\
\hline 198 & Q5GAQ3 & Putative alanine aminotransferase & Z. mays & 10 & 220 & 0.09 & 5.80 & 57003 & amino acid metabolism \\
\hline 199 & B6SUH6 & Non-cyanogenic B.-glucosidase & Z. mays & 27 & 66 & 0.09 & 11.14 & 56909 & misc \\
\hline 200 & F2D9E0 & Predicted protein & H. vulgare & 4 & 120 & 0.09 & 5.64 & 28037 & protein \\
\hline 201 & F2DGF3 & Predicted protein & H. vulgare & 10 & 145 & 0.09 & 6.81 & 115601 & TCA \\
\hline 202 & $\mathrm{O} 23963$ & Elongation factor $\mathrm{Tu}$ & G. $\max$ & 12 & 111 & 0.09 & 5.14 & 38044 & protein \\
\hline 203 & A9PE52 & Gamma class glutathione transferase EF1Bgamma2 & P. trichocarpa & 8 & 128 & 0.09 & 6.04 & 48127 & protein \\
\hline 204 & $\mathrm{O} 22143$ & At2g45290/F4L23.20 & A. thaliana & 12 & 167 & 0.08 & 5.96 & 69264 & OPP \\
\hline 205 & B4FQM2 & Pyrophosphate--fructose 6-phosphate 1-phosphotransferase B. subunit & Z. mays & 7 & 110 & 0.08 & 6.35 & 61607 & glycolysis \\
\hline 206 & H9A1W2 & Acyl-activating enzyme 10 & C. sativa & 4 & 110 & 0.08 & 6.49 & 62013 & lipid metabolism \\
\hline 207 & $\mathrm{O} 24135$ & Citrate synthase & N.tabacum & 7 & 188 & 0.08 & 7.85 & 52744 & TCA \\
\hline 208 & Q93YG1 & Monodehydroascorbate reductase & M. crystallinum & 5 & 111 & 0.08 & 6.81 & 51944 & redox \\
\hline 209 & B9GXZ8 & Pectinesterase & P. trichocarpa & 6 & 77 & 0.08 & 8.98 & 63820 & cell wall \\
\hline 210 & Q40270 & RNA-binding protein & M. crystallinum & 2 & 41 & 0.08 & 4.79 & 32001 & RNA \\
\hline 211 & Q9SIR5 & Expressed protein & A. thaliana & 4 & 42 & 0.08 & 6.84 & 32851 & not assigned \\
\hline 212 & K3XWI6 & Uncharacterized protein & S. italica & 12 & 208 & 0.07 & 6.60 & 55489 & glycolysis \\
\hline 213 & B9HJ23 & Predicted protein & P. trichocarpa & 11 & 195 & 0.07 & 6.54 & 101700 & misc \\
\hline 214 & A7WPL0 & Putative reductase & N.tabacum & 8 & 99 & 0.07 & 8.09 & 44428 & TCA \\
\hline 215 & A8JCE1 & Predicted protein & C. reinhardtii & 17 & 36 & 0.07 & 7.94 & 33842 & protein \\
\hline 216 & F2E397 & Predicted protein & H. vulgare & 4 & 134 & 0.07 & 5.87 & 34933 & major $\mathrm{CHO}$ metabolism \\
\hline 217 & K3XKD1 & Uncharacterized protein & S. italica & 4 & 86 & 0.07 & 6.76 & 34429 & protein \\
\hline 218 & F2D269 & Predicted protein & H. vulgare & 13 & 185 & 0.07 & 5.04 & 73306 & stress \\
\hline 219 & K3XJZ1 & Uncharacterized protein & S. italica & 6 & 170 & 0.07 & 9.39 & 35932 & protein \\
\hline 220 & Q9XEN8 & Annexin & N.tabacum & 5 & 44 & 0.07 & 5.53 & 36029 & cell \\
\hline 221 & F2DAD8 & Predicted protein & H. vulgare & 2 & 78 & 0.07 & 7.15 & 50084 & amino acid metabolism \\
\hline 222 & Q43057 & Aspartate aminotransferase & P. miliaceum & 6 & 49 & 0.07 & 8.70 & 48154 & amino acid metabolism \\
\hline 223 & K3Y7B0 & Uncharacterized protein & S. italica & 17 & 60 & 0.07 & 7.33 & 48942 & TCA \\
\hline
\end{tabular}




\begin{tabular}{|c|c|c|c|c|c|c|c|c|c|}
\hline 224 & Q9SUU0 & Serine hydroxymethyltransferase & A. thaliana & 6 & 85 & 0.07 & 8.05 & 50988 & C1-metabolism \\
\hline 225 & C8YNG6 & 3-ketoacyl CoA thiolase 1 & P. hybrida & 10 & 96 & 0.06 & 8.29 & 49405 & amino acid metabolism \\
\hline 226 & B9RI89 & Serine-threonine protein kinase, plant-type, putative & R. communis & 2 & 51 & 0.06 & 8.18 & 40375 & not assigned \\
\hline 227 & Q39986 & Cysteine proteinase & Hemerocallis sp. & 4 & 87 & 0.06 & 5.50 & 40006 & protein \\
\hline 228 & A0FKE6 & Chloroplast threonine deaminase 1 & S. lycopersicum & 4 & 38 & 0.06 & 6.37 & 66653 & amino acid metabolism \\
\hline 229 & K4A6V8 & Uncharacterized protein & S. italica & 7 & 121 & 0.06 & 7.03 & 70549 & glycolysis \\
\hline 230 & K3YRV8 & Uncharacterized protein & S. italica & 9 & 109 & 0.06 & 7.27 & 55802 & TCA \\
\hline 231 & B4FYM0 & Acetyl-CoA acetyltransferase, cytosolic 2 & Z. mays & 8 & 271 & 0.06 & 4.88 & 43450 & secondary metabolism \\
\hline 232 & A8I7T1 & Heat shock protein 90B & C. reinhardtii & 5 & 117 & 0.06 & 7.66 & 87547 & stress \\
\hline 233 & E5GCI7 & Ornithine carbamoyltransferase & C. melo & 2 & 127 & 0.06 & 8.40 & 42175 & amino acid metabolism \\
\hline 234 & Q42953 & Alcohol dehydrogenase & N.tabacum & 8 & 54 & 0.06 & 7.05 & 41981 & fermentation \\
\hline 235 & А9P9A2 & Putative uncharacterized protein & P. trichocarpa & 15 & 209 & 0.05 & 9.36 & 46272 & RNA \\
\hline 236 & B7SA66 & Hsp90-like protein & D. glomerata & 11 & 138 & 0.05 & 4.97 & 92918 & stress \\
\hline 237 & F2DDZ1 & Predicted protein & H. vulgare & 2 & 116 & 0.05 & 6.42 & 46133 & nucleotide metabolism \\
\hline 238 & Q42737 & NADP-malate dehydrogenase & F. trinervia & 3 & 54 & 0.05 & 6.46 & 46155 & TCA \\
\hline 239 & Q6L619 & B.-galactosidase & R. sativus & 4 & 95 & 0.05 & 8.38 & 93664 & misc \\
\hline 240 & Q8H6B6 & Chaperone protein HtpG & $X$. viscosa & 22 & 146 & 0.05 & 5.02 & 92957 & stress \\
\hline 241 & A6XMY8 & Methylenetetrahydrofolate reductase & T. monococcum & 13 & 146 & 0.05 & 6.20 & 65461 & C1-metabolism \\
\hline 242 & G8HAB1 & PLP-dependent aminotransferase & P. somniferum & 3 & 57 & 0.05 & 7.09 & 50587 & secondary metabolism \\
\hline 243 & K3XHS0 & Uncharacterized protein & S. italica & 2 & 37 & 0.05 & 5.81 & 48047 & hormone metabolism \\
\hline 244 & Q9S768 & Alanine aminotransferase & O. sativa & 5 & 283 & 0.04 & 6.55 & 53130 & amino acid metabolism \\
\hline 245 & B3H621 & Peptidase M1 family protein & A. thaliana & 5 & 121 & 0.04 & 6.79 & 106723 & protein \\
\hline 246 & K4A938 & Uncharacterized protein & S. italica & 4 & 73 & 0.04 & 6.65 & 53547 & nucleotide metabolism \\
\hline 247 & Q38766 & Victorin binding protein & A. sativa & 8 & 148 & 0.04 & 6.92 & 112298 & photosynthesis \\
\hline 248 & B6THZ8 & Threonine synthase & Z. mays & 4 & 50 & 0.04 & 5.54 & 58091 & amino acid metabolism \\
\hline 249 & E4MX84 & mRNA, clone: RTFL01-14-C02 & T. halophila & 2 & 30 & 0.04 & 6.35 & 55142 & protein \\
\hline 250 & F2EAT2 & Pyruvate kinase & H. vulgare & 2 & 40 & 0.04 & 7.56 & 55816 & glycolysis \\
\hline 251 & K3YH65 & Uncharacterized protein & S. italica & 2 & 40 & 0.04 & 6.96 & 56313 & amino acid metabolism \\
\hline 252 & B5BLW2 & Carotenoid cleavage dioxygenase 1 & M. truncatula & 2 & 55 & 0.04 & 5.69 & 61063 & secondary metabolism \\
\hline 253 & F2CTR2 & Predicted protein & H. vulgare & 3 & 120 & 0.04 & 6.79 & 59167 & protein \\
\hline 254 & F2DUH3 & Predicted protein & H. vulgare & 4 & 53 & 0.04 & 6.99 & 60964 & protein \\
\hline 255 & Q0W9E2 & B. chaperonin 60 & S. commersonii & 5 & 63 & 0.04 & 5.63 & 63333 & protein \\
\hline
\end{tabular}




\begin{tabular}{|c|c|c|c|c|c|c|c|c|c|}
\hline 256 & $\mathrm{~B} 2 \mathrm{Z} 6 \mathrm{P} 0$ & 4-Coumarate:CoA ligase & P. trichocarpa & 2 & 66 & 0.04 & 7.71 & 59541 & secondary metabolism \\
\hline 257 & F2WMV4 & Acetyl-CoA carboxylase BC subunit & J. curcas & 6 & 76 & 0.04 & 7.27 & 58747 & lipid metabolism \\
\hline 258 & K3YKV0 & Uncharacterized protein & S. italica & 18 & 221 & 0.04 & 5.54 & 87462 & cell \\
\hline 259 & Q8L5C2 & $110 \mathrm{kDa} 4 \mathrm{SNc}$-Tudor domain protein & P. sativum & 7 & 82 & 0.04 & 7.36 & 108600 & RNA \\
\hline 260 & B2KNE6 & Phospholipase D & H. annuиs & 15 & 170 & 0.03 & 5.52 & 92315 & lipid metabolism \\
\hline 261 & F2DIS4 & Malic enzyme & H. vulgare & 2 & 57 & 0.03 & 7.40 & 68411 & TCA \\
\hline 262 & J9Z414 & B.-D-glucan exohydolase & E. guineensis & 2 & 109 & 0.03 & 7.88 & 69462 & cell wall \\
\hline 263 & Q2LFC1 & AGO4-2 & N. benthamiana & 5 & 37 & 0.03 & 8.78 & 102331 & RNA \\
\hline 264 & K3ZH11 & Uncharacterized protein & S. italica & 12 & 61 & 0.03 & 5.38 & 118046 & protein \\
\hline 265 & K3YPS9 & Uncharacterized protein & S. italica & 13 & 134 & 0.03 & 5.43 & 97789 & protein \\
\hline 266 & Q9XFX7 & Phospholipase D & C. plantagineum & 4 & 36 & 0.03 & 5.24 & 92228 & lipid metabolism \\
\hline 267 & B6U237 & Heat shock $70 \mathrm{kDa}$ protein 4 & Z. mays & 7 & 115 & 0.03 & 5.66 & 94309 & stress \\
\hline 268 & F2DBR2 & Predicted protein & H. vulgare & 2 & 23 & 0.02 & 5.20 & 112909 & protein \\
\hline 269 & K3ZH26 & Uncharacterized protein & S. italica & 10 & 54 & 0.02 & 6.07 & 120414 & protein \\
\hline 270 & Q39834 & Clathrin heavy chain & G. $\max$ & 8 & 72 & 0.01 & 5.55 & 194430 & cell \\
\hline 271 & В3ТМ07 & Ribosomal protein L17 & E. guineensis & 11 & 36 & - & 10.49 & 20744 & protein \\
\hline 272 & E4MXV2 & mRNA, clone: RTFL01-41-F15 & T. halophila & 10 & 75 & - & 6.09 & 64768 & secondary metabolism \\
\hline
\end{tabular}

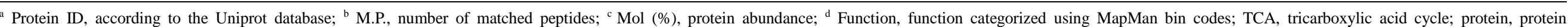

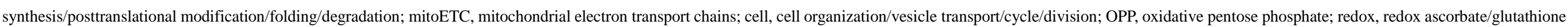
metabolism; DNA, DNA synthesis/repair; RNA, RNA processing/regulation of transcription; and misc, miscellaneous. 
Supplemental Table 7. Proteins Identified in L. japonica Immature Flower Buds at Fraction 1 using Polyethylene Glycol Fractionation.

\begin{tabular}{|c|c|c|c|c|c|c|c|c|c|}
\hline No. & Protein ID ${ }^{\text {a }}$ & Description & Species & M.P. ${ }^{b}$ & Score & $\operatorname{Mol}(\%)^{\mathrm{c}}$ & $\mathrm{pI}$ & Mass (Da) & Function $^{\mathrm{d}}$ \\
\hline 1 & Q2TFP1 & Tubulin B4 & G. $\max$ & 118 & 2374 & 1.52 & 4.83 & 51083 & cell \\
\hline 2 & B5M4B1 & Beta-tubulin & S. tuberosum & 95 & 1872 & 1.30 & 4.86 & 51166 & cell \\
\hline 3 & Q9ZQT0 & Actin & G. echinata & 30 & 707 & 1.25 & 6.19 & 17082 & cell \\
\hline 4 & K3Z679 & Uncharacterized protein & S. italica & 113 & 1832 & 1.16 & 4.88 & 50728 & cell \\
\hline 5 & Q8MCW3 & Ribulose bisphosphate carboxylase large chain & L. coelhoi & 65 & 1114 & 1.11 & 8.88 & 34008 & photosynthesis \\
\hline 6 & Q32121 & Ribulose bisphosphate carboxylase large chain & D. californica & 74 & 1208 & 0.95 & 8.18 & 40464 & photosynthesis \\
\hline 7 & C4B8E5 & Glyceraldehyde-3-phosphate dehydrogenase & T. gesneriana & 94 & 3314 & 0.94 & 7.96 & 20964 & glycolysis \\
\hline 8 & F2YP47 & Beta-tubulin & V. planifolia & 68 & 1217 & 0.89 & 4.87 & 50673 & cell \\
\hline 9 & H1ZY49 & Translation elongation factor 1-alpha & L. maackii & 33 & 467 & 0.82 & 5.00 & 11619 & protein \\
\hline 10 & В3ТМ39 & Ribosomal protein S14 & E. guineensis & 11 & 232 & 0.65 & 10.70 & 16428 & protein \\
\hline 11 & Q6PKU2 & Putative glyceraldehyde-3-phosphate dehydrogenase & O. minor & 60 & 1849 & 0.65 & 7.09 & 22122 & glycolysis \\
\hline 12 & Q0ZUN6 & Alpha-tubulin & C. scutata & 81 & 1802 & 0.65 & 6.19 & 44358 & cell \\
\hline 13 & O03843 & Ribulose-bisphosphate carboxylase & T. glandulifera & 114 & 1828 & 0.64 & 6.04 & 53346 & photosynthesis \\
\hline 14 & Q8H6N0 & Beta-tubulin 1 & G. hirsutum & 89 & 1476 & 0.61 & 4.87 & 50441 & cell \\
\hline 15 & Q7XAP7 & Glyceraldehyde-3-phosphate dehydrogenase & H. cordata & 59 & 2207 & 0.57 & 8.34 & 24077 & glycolysis \\
\hline 16 & $\mathrm{O} 23930$ & Phosphoenolpyruvate carboxylase & $F$. pringlei & 5 & 197 & 0.57 & 4.59 & 10308 & glycolysis \\
\hline 17 & Q9SAQ0 & ATP synthase subunit B. & N. sylvestris & 35 & 1110 & 0.55 & 6.06 & 59597 & mitoETC \\
\hline 18 & B6TJ90 & Histone $\mathrm{H} 4$ & Z. mays & 15 & 173 & 0.54 & 9.67 & 20120 & cell \\
\hline 19 & J9QAL9 & Ribosomal protein L2 & A. corniculatum & 13 & 343 & 0.54 & 10.23 & 21703 & protein \\
\hline 20 & O82564 & Actin 2 & A. phyllitidis & 56 & 706 & 0.53 & 5.49 & 41828 & cell \\
\hline 21 & F2E3Y2 & Predicted protein & H. vulgare & 77 & 1694 & 0.53 & 5.16 & 65885 & cell \\
\hline 22 & Q5KTY2 & Ribulose-1,5-bisphosphate carboxylase large subunit & A. sutepensis & 83 & 1025 & 0.53 & 6.92 & 51143 & photosynthesis \\
\hline 23 & A2Q5W0 & Alpha-tubulin & M. truncatula & 67 & 1809 & 0.47 & 5.10 & 50457 & cell \\
\hline 24 & K4A9V6 & Uncharacterized protein & S. italica & 58 & 1077 & 0.47 & 5.10 & 50519 & cell \\
\hline 25 & Q8L4S4 & Phosphoglycerate kinase & A. speltoides & 22 & 261 & 0.46 & 5.01 & 31433 & photosynthesis \\
\hline 26 & A7KQH0 & B.-tubulin & E. grandis & 81 & 1267 & 0.45 & 4.94 & 50615 & cell \\
\hline 27 & K4ABP3 & Uncharacterized protein & S. italica & 28 & 379 & 0.44 & 6.13 & 41769 & cell wall \\
\hline 28 & B3TLP4 & Ribosomal protein L30 & E. guineensis & 5 & 162 & 0.44 & 9.72 & 12451 & protein \\
\hline 29 & F2D6I8 & Predicted protein & H. vulgare & 69 & 2643 & 0.43 & 7.14 & 36874 & glycolysis \\
\hline 30 & B4FP40 & ADP-ribosylation factor & Z. mays & 9 & 204 & 0.41 & 6.95 & 20710 & protein \\
\hline 31 & F8WLB7 & $40 \mathrm{~S}$ ribosomal protein $\mathrm{S} 3$ & C. unshiu & 10 & 130 & 0.37 & 9.63 & 20599 & protein \\
\hline
\end{tabular}




\begin{tabular}{|c|c|c|c|c|c|c|c|c|c|}
\hline 32 & A6YIK6 & Alpha-tubulin 4 & P. tremuloides & 46 & 733 & 0.36 & 5.01 & 50481 & cell \\
\hline 33 & H9NIE1 & Cell division cycle protein 48 & C. sinensis & 79 & 1861 & 0.36 & 5.29 & 90644 & cell \\
\hline 34 & D6PPS8 & AT3G02360-like protein & C. grandiflora & 7 & 275 & 0.36 & 9.22 & 21010 & OPP \\
\hline 35 & Q08II7 & Heat shock protein 70 -like protein & L. sativa & 4 & 291 & 0.35 & 10.33 & 12539 & stress \\
\hline 36 & J3RTS9 & Glyceraldehyde-3-phosphate dehydrogenase & C. pentagona & 120 & 3365 & 0.34 & 7.23 & 32382 & glycolysis \\
\hline 37 & B6T9G3 & Alpha-1,4-glucan-protein synthase 1 & Z. mays & 26 & 499 & 0.34 & 7.05 & 41278 & cell wall \\
\hline 38 & Q8VWP3 & Glyceraldehyde-3-phosphate dehydrogenase & C. аппиит & 17 & 184 & 0.33 & 6.70 & 33465 & photosynthesis \\
\hline 39 & Q9AYS9 & Tat binding protein like protein & B. campestris & 30 & 724 & 0.33 & 5.03 & 47733 & protein \\
\hline 40 & F2CQQ3 & Predicted protein & H. vulgare & 18 & 688 & 0.33 & 5.16 & 54310 & transport \\
\hline 41 & F2DSZ0 & Predicted protein & H. vulgare & 32 & 617 & 0.33 & 5.14 & 50087 & cell \\
\hline 42 & E4MXI2 & mRNA, clone: RTFL01-39-D20 & T. halophila & 48 & 999 & 0.33 & 5.21 & 73890 & stress \\
\hline 43 & B6EBD6 & Heat shock protein $90-2$ & G. $\max$ & 51 & 851 & 0.32 & 5.07 & 80392 & stress \\
\hline 44 & Q5ZF83 & Putative uncharacterized protein & P. major & 14 & 224 & 0.32 & 7.28 & 25390 & cell wall \\
\hline 45 & D0EJY9 & Molecular chaperone Hsp90-3 & N. benthamiana & 38 & 732 & 0.30 & 5.02 & 80638 & stress \\
\hline 46 & F2DR86 & Predicted protein & H. vulgare & 42 & 429 & 0.29 & 5.48 & 41914 & cell \\
\hline 47 & E5GBV8 & Glyceraldehyde-3-phosphate dehydrogenase & C. melo & 62 & 2356 & 0.29 & 7.12 & 36754 & glycolysis \\
\hline 48 & Q9XEW9 & Elongation factor 1-alpha & L. longiflorum & 45 & 411 & 0.29 & 9.07 & 49711 & protein \\
\hline 49 & B2VQE0 & Methionine synthase & O. ramosa & 55 & 726 & 0.27 & 6.40 & 84862 & amino acid metabolism \\
\hline 50 & K3YQW5 & Uncharacterized protein & S. italica & 21 & 599 & 0.27 & 5.39 & 68637 & transport \\
\hline 51 & Q45Q23 & PHB2 & N. benthamiana & 7 & 170 & 0.27 & 9.50 & 31837 & mitoETC \\
\hline 52 & Q34691 & ATP synthase subunit alpha & H. annuиs & 33 & 516 & 0.27 & 6.60 & 55766 & mitoETC \\
\hline 53 & E4MVY3 & mRNA, clone: RTFL01-01-M18 & T. halophila & 54 & 1191 & 0.26 & 6.25 & 94734 & protein \\
\hline 54 & F2DAU4 & Predicted protein & H. vulgare & 39 & 678 & 0.26 & 7.56 & 62696 & protein \\
\hline 55 & Q43833 & Glyceraldehyde 3-phosphate dehydrogenase & S. tuberosum & 51 & 1238 & 0.26 & 6.44 & 32598 & glycolysis \\
\hline 56 & A2V880 & G protein B.-subunit-like protein & N. tabacum & 14 & 405 & 0.26 & 7.09 & 16100 & signalling \\
\hline 57 & A9PD17 & Predicted protein & P. trichocarpa & 35 & 615 & 0.25 & 5.82 & 47103 & protein \\
\hline 58 & K3XX30 & Uncharacterized protein & S. italica & 30 & 630 & 0.25 & 5.07 & 47991 & protein \\
\hline 59 & B3TLQ8 & Ribosomal protein & E. guineensis & 11 & 327 & 0.25 & 9.79 & 24908 & protein \\
\hline 60 & K3ZVI3 & Uncharacterized protein & S. italica & 11 & 271 & 0.25 & 5.16 & 33393 & protein \\
\hline 61 & A8MS83 & $60 \mathrm{~S}$ ribosomal protein $\mathrm{L} 23 \mathrm{a}-2$ & A. thaliana & 13 & 127 & 0.25 & 10.17 & 16733 & protein \\
\hline 62 & Q84RD8 & Adenosylhomocysteinase & M. truncatula & 43 & 629 & 0.25 & 6.01 & 53790 & amino acid metabolism \\
\hline 63 & B1PBX8 & Putative uncharacterized protein & A. lyrata & 11 & 243 & 0.24 & 8.94 & 19793 & protein \\
\hline
\end{tabular}




\begin{tabular}{|c|c|c|c|c|c|c|c|c|c|}
\hline 64 & D6PPX2 & AT3G08530-like protein & N. paniculata & 7 & 150 & 0.24 & 4.56 & 20434 & cell \\
\hline 65 & Q1WFH5 & Phosphoenolpyruvate carboxylase isoform 3 & C. hilariana & 17 & 139 & 0.23 & 8.94 & 41462 & glycolysis \\
\hline 66 & K4ACE3 & Uncharacterized protein & S. italica & 19 & 171 & 0.23 & 6.09 & 35803 & TCA \\
\hline 67 & B6SJZ8 & Glyceraldehyde-3-phosphate dehydrogenase, cytosolic & Z. mays & 57 & 2135 & 0.22 & 6.96 & 36614 & glycolysis \\
\hline 68 & K3ZTA6 & Uncharacterized protein & S. italica & 26 & 473 & 0.22 & 6.21 & 49589 & protein \\
\hline 69 & K3Y516 & Uncharacterized protein & S. italica & 41 & 803 & 0.22 & 6.61 & 102308 & protein \\
\hline 70 & D0ELH5 & Peptidyl-prolyl cis-trans isomerase & C. sinensis & 3 & 102 & 0.22 & 8.46 & 18310 & cell \\
\hline 71 & Q9M6B3 & Malate dehydrogenase & V. vinifera & 15 & 419 & 0.22 & 8.62 & 37137 & TCA \\
\hline 72 & Q1KUM7 & Putative uncharacterized protein & C. spinosa & 20 & 280 & 0.22 & 6.62 & 59525 & protein \\
\hline 73 & B4F8L7 & Glyceraldehyde-3-phosphate dehydrogenase B & Z. mays & 22 & 173 & 0.21 & 6.39 & 47663 & photosynthesis \\
\hline 74 & Q1KUX5 & 6-phosphogluconate dehydrogenase, decarboxylating & C. spinosa & 25 & 435 & 0.21 & 6.06 & 53777 & OPP \\
\hline 75 & D7NHW9 & 2-phospho-D-glycerate hydrolase & P. trifoliata & 18 & 453 & 0.21 & 5.78 & 47986 & glycolysis \\
\hline 76 & B6T416 & Ribulose bisphosphate carboxylase/oxygenase activase & Z. mays & 28 & 985 & 0.21 & 6.73 & 48079 & photosynthesis \\
\hline 77 & E4MXI1 & mRNA, clone: RTFL01-39-B07 & T. halophila & 19 & 295 & 0.21 & 6.98 & 48063 & photosynthesis \\
\hline 78 & B3TM23 & 40S ribosomal protein $\mathrm{S} 4$ & E. guineensis & 15 & 148 & 0.21 & 10.24 & 30023 & protein \\
\hline 79 & Q8LFN3 & Putative chaperonin & A. thaliana & 21 & 165 & 0.21 & 6.21 & 59466 & protein \\
\hline 80 & D4N5G0 & Alpha-form rubisco activase & G. $\max$ & 35 & 946 & 0.21 & 6.25 & 52531 & photosynthesis \\
\hline 81 & F2DJQ0 & Predicted protein & H. vulgare & 16 & 261 & 0.20 & 7.72 & 36458 & cell wall \\
\hline 82 & H6VP90 & ATP synthase subunit B. & C. lobatus & 14 & 263 & 0.20 & 5.19 & 47813 & photosynthesis \\
\hline 83 & E1Z5R3 & Putative uncharacterized protein & C. variabilis & 47 & 1069 & 0.20 & 5.12 & 93194 & cell \\
\hline 84 & G5DVX2 & Phosphoglycerate kinase & S. latifolia & 25 & 506 & 0.19 & 6.58 & 51352 & photosynthesis \\
\hline 85 & K3XFR6 & Uncharacterized protein & S. italica & 17 & 286 & 0.19 & 7.46 & 66448 & TCA \\
\hline 86 & K3ZXL7 & Eukaryotic translation initiation factor $5 \mathrm{~A}$ & S. italica & 4 & 115 & 0.19 & 5.99 & 17613 & protein \\
\hline 87 & Q7XTK9 & Catalase & P. persica & 20 & 422 & 0.19 & 7.25 & 57262 & redox \\
\hline 88 & D2KZ81 & S-adenosylmethionine synthase & M. domestica & 7 & 333 & 0.19 & 4.94 & 24831 & amino acid metabolism \\
\hline 89 & K3XX43 & Uncharacterized protein & S. italica & 21 & 404 & 0.18 & 6.29 & 48119 & protein \\
\hline 90 & G8GJ68 & Adenosylhomocysteinase & L. usitatissimum & 34 & 587 & 0.18 & 5.99 & 61872 & amino acid metabolism \\
\hline 91 & Q75ZE0 & 14-3-3 e-1 protein & N. tabacum & 9 & 145 & 0.18 & 4.78 & 29479 & signalling \\
\hline 92 & K3Z913 & Uncharacterized protein & S. italica & 5 & 131 & 0.18 & 9.47 & 26793 & cell \\
\hline 93 & $\mathrm{O} 23254$ & Serine hydroxymethyltransferase & A. thaliana & 23 & 441 & 0.18 & 7.23 & 52141 & C1-metabolism \\
\hline 94 & B3TLN9 & Cytoplasmic ribosomal protein $\mathrm{S} 15 \mathrm{a}$ & E. guineensis & 4 & 46 & 0.17 & 9.89 & 14862 & protein \\
\hline 95 & A0ZQA9 & ATP synthase subunit B. & P. baccatum & 18 & 255 & 0.17 & 5.40 & 52897 & photosynthesis \\
\hline
\end{tabular}




\begin{tabular}{|c|c|c|c|c|c|c|c|c|c|}
\hline 96 & B6TI65 & Fructose-bisphosphate aldolase & Z. mays & 7 & 345 & 0.17 & 7.83 & 41924 & photosynthesis \\
\hline 97 & Q5D6C3 & Adenosylhomocysteinase & A. thaliana & 51 & 772 & 0.17 & 6.10 & 53970 & amino acid metabolism \\
\hline 98 & F2DX25 & Predicted protein & H. vulgare & 27 & 446 & 0.17 & 5.06 & 80668 & stress \\
\hline 99 & B6THG9 & $60 \mathrm{~S}$ ribosomal protein $\mathrm{L} 5-1$ & Z. mays & 13 & 338 & 0.17 & 9.32 & 34462 & protein \\
\hline 100 & A1YUL9 & Importin subunit alpha & N. benthamiana & 17 & 363 & 0.17 & 5.38 & 59114 & protein \\
\hline 101 & Q9AXR6 & ATP:citrate lyase & C. аппиит & 22 & 502 & 0.17 & 7.39 & 66376 & TCA \\
\hline 102 & Q8H945 & Phosphoenolpyruvate carboxylase & L. japonicus & 43 & 527 & 0.17 & 5.88 & 111020 & glycolysis \\
\hline 103 & Q5MFD0 & ATPase subunit 8 & L. Bergthorsson & 3 & 60 & 0.16 & 8.84 & 15817 & mitoETC \\
\hline 104 & B4FD78 & $60 \mathrm{~S}$ acidic ribosomal protein $\mathrm{P} 2 \mathrm{~B}$ & Z. mays & 11 & 300 & 0.16 & 4.27 & 11693 & protein \\
\hline 105 & B3TLL5 & 40S ribosomal protein S19 & E. guineensis & 12 & 44 & 0.16 & 10.04 & 16202 & protein \\
\hline 106 & B5LAU5 & DH putative B.-hydroxyacyl-ACP dehydratase & С. аппиит & 9 & 48 & 0.16 & 9.29 & 24080 & lipid metabolism \\
\hline 107 & Q9FE12 & Peroxiredoxin & P. vulgaris & 10 & 178 & 0.16 & 5.33 & 28776 & redox \\
\hline 108 & K3Z5U9 & Uncharacterized protein & S. italica & 19 & 200 & 0.15 & 6.47 & 49826 & photosynthesis \\
\hline 109 & $\mathrm{O} 48903$ & Malate dehydrogenase & M. sativa & 16 & 171 & 0.15 & 8.29 & 38397 & gluconeogenesis \\
\hline 110 & B6TDT1 & $26 \mathrm{~S}$ protease regulatory subunit $6 \mathrm{~B}$ & Z. mays & 18 & 305 & 0.15 & 6.07 & 46827 & protein \\
\hline 111 & Q9SRV5 & 5-methyltetrahydropteroyltriglutamate--homocysteine methyltransferase & A. thaliana & 34 & 477 & 0.15 & 6.51 & 84873 & amino acid metabolism \\
\hline 112 & G9FCD2 & ATP synthase subunit alpha, chloroplastic & B. hygrometrica & 19 & 319 & 0.15 & 5.34 & 55392 & photosynthesis \\
\hline 113 & K3XI07 & Uncharacterized protein & S. italica & 11 & 247 & 0.15 & 8.73 & 47392 & cell wall \\
\hline 114 & B5LAW6 & Putative S-adenosylmethionine synthetase & C. аппиит & 14 & 229 & 0.15 & 6.05 & 43056 & amino acid metabolism \\
\hline 115 & В3ТМ36 & 40 S ribosomal protein $\mathrm{S} 8$ & E. guineensis & 3 & 175 & 0.15 & 10.43 & 25347 & protein \\
\hline 116 & Q7XJH9 & Transaldolase & S. lycopersicum & 10 & 248 & 0.15 & 5.96 & 48062 & OPP \\
\hline 117 & Q6B6L9 & UDP-D-glucuronate decarboxylase & H. vulgare & 10 & 346 & 0.15 & 8.76 & 44245 & cell wall \\
\hline 118 & F2DLC2 & Proteasome subunit alpha type & H. vulgare & 6 & 300 & 0.14 & 4.68 & 26087 & protein \\
\hline 119 & Q9ATF4 & Ribosomal protein $\mathrm{L} 33$ & C. sativa & 5 & 148 & 0.14 & 10.58 & 12831 & protein \\
\hline 120 & K3XH89 & Uncharacterized protein & S. italica & 19 & 310 & 0.14 & 5.87 & 52978 & OPP \\
\hline 121 & Q6ZXH7 & Putative ascorbate peroxidase & P. canadensis & 7 & 197 & 0.14 & 4.97 & 22506 & redox \\
\hline 122 & Q9SH69 & 6-phosphogluconate dehydrogenase, decarboxylating & A. thaliana & 14 & 474 & 0.14 & 5.45 & 53686 & OPP \\
\hline 123 & K3XL78 & Triosephosphate isomerase & S. italica & 7 & 162 & 0.14 & 5.49 & 27278 & glycolysis \\
\hline 124 & F2D0V3 & Predicted protein & H. vulgare & 11 & 116 & 0.14 & 9.61 & 32911 & transport \\
\hline 125 & $\mathrm{H} 2 \mathrm{BC} 31$ & 50S ribosomal protein L14, chloroplastic & B. liliputana & 2 & 160 & 0.14 & 10.32 & 13665 & protein \\
\hline 126 & B6TGB2 & Elongation factor 1-gamma 3 & Z. mays & 12 & 288 & 0.13 & 6.37 & 47357 & protein \\
\hline 127 & A0FKE6 & Chloroplast threonine deaminase 1 & S. lycopersicum & 20 & 387 & 0.13 & 6.37 & 66653 & amino acid metabolism \\
\hline
\end{tabular}




\begin{tabular}{|c|c|c|c|c|c|c|c|c|c|}
\hline 128 & F2DRC5 & Predicted protein & H. vulgare & 20 & 517 & 0.13 & 5.92 & 57700 & protein \\
\hline 129 & K3Z3J3 & Uncharacterized protein & S. italica & 34 & 482 & 0.13 & 6.64 & 105687 & transport \\
\hline 130 & B9N744 & Gamma class glutathione transferase EF1Bgamma3 & P. trichocarpa & 13 & 292 & 0.13 & 6.05 & 48353 & protein \\
\hline 131 & Q8GZR6 & GcpE & S. lycopersicum & 29 & 726 & 0.13 & 6.11 & 82805 & secondary metabolism \\
\hline 132 & F2Z9R1 & Glucose-6-phosphate 1-dehydrogenase & $N$. benthamiana & 18 & 141 & 0.13 & 6.49 & 58894 & OPP \\
\hline 133 & E1ZP98 & Putative uncharacterized protein & C. variabilis & 18 & 305 & 0.13 & 5.74 & 93462 & protein \\
\hline 134 & A8JGV6 & 14-3-3 protein & C. reinhardtii & 10 & 164 & 0.13 & 5.00 & 29667 & signalling \\
\hline 135 & Q4FGD9 & ATP synthase epsilon chain, chloroplastic & G. biloba & 2 & 106 & 0.12 & 6.11 & 14962 & photosynthesis \\
\hline 136 & F2D448 & Predicted protein & H. vulgare & 13 & 273 & 0.12 & 10.17 & 30607 & protein \\
\hline 137 & B5TM95 & NADH dehydrogenase subunit 7 & A. thaliana & 10 & 223 & 0.12 & 7.12 & 45555 & mitoETC \\
\hline 138 & K4A7I4 & Uncharacterized protein & S. italica & 15 & 393 & 0.12 & 5.40 & 61666 & photosynthesis \\
\hline 139 & J9XLE5 & Putative isopentenyl diphosphate isomerase & O. europaea & 11 & 111 & 0.12 & 5.38 & 25822 & secondary metabolism \\
\hline 140 & D3GQL2 & Aconitate hydratase 2 & C. clementina & 48 & 519 & 0.12 & 6.54 & 98475 & TCA \\
\hline 141 & O24511 & Catalase & N. tabacum & 19 & 236 & 0.12 & 7.33 & 57225 & redox \\
\hline 142 & K3ZD99 & Uncharacterized protein & S. italica & 3 & 90 & 0.12 & 9.16 & 26325 & transport \\
\hline 143 & B5LAU9 & Glutamine synthetase & C. аппиит & 15 & 169 & 0.12 & 6.93 & 47926 & N-metabolism \\
\hline 144 & F2EKH0 & Predicted protein & H. vulgare & 6 & 276 & 0.12 & 4.60 & 31771 & protein \\
\hline 145 & K3YR06 & Uncharacterized protein & S. italica & 11 & 356 & 0.12 & 6.16 & 64225 & protein \\
\hline 146 & B4G1C9 & Dihydrolipoyllysine-residue acetyltransferase component of pyruvatedehydrogenase & Z. mays & 9 & 216 & 0.12 & 8.10 & 47858 & TCA \\
\hline 147 & G5DXP1 & Fructose-bisphosphate aldolase & S. latifolia & 7 & 291 & 0.12 & 6.16 & 42771 & photosynthesis \\
\hline 148 & K3Y7B0 & Uncharacterized protein & S. italica & 25 & 151 & 0.12 & 8.70 & 48942 & TCA \\
\hline 149 & K3YH95 & Uncharacterized protein & S. italica & 15 & 229 & 0.12 & 7.05 & 53771 & glycolysis \\
\hline 150 & Q39834 & Clathrin heavy chain & G. $\max$ & 59 & 725 & 0.12 & 5.55 & 194430 & cell \\
\hline 151 & E5GBS0 & 40S ribosomal protein S24 & C. melo & 2 & 93 & 0.12 & 10.64 & 15761 & protein \\
\hline 152 & F2D861 & Predicted protein & H. vulgare & 6 & 245 & 0.12 & 10.43 & 15765 & protein \\
\hline 153 & Q9C8P0 & $\begin{array}{l}\text { Dihydrolipoyllysine-residue acetyltransferase component } 5 \text { of pyruvate } \\
\text { dehydrogenase complex, chloroplastic }\end{array}$ & A. thaliana & 9 & 276 & 0.12 & 8.78 & 48334 & $\mathrm{TCA}$ \\
\hline 154 & G5DXC9 & $26 \mathrm{~S}$ proteasome regulatory subunit $\mathrm{N} 1$ & S. latifolia & 19 & 502 & 0.11 & 5.16 & 97731 & protein \\
\hline 155 & В6Т3Р9 & Enolase & Z. mays & 8 & 291 & 0.11 & 5.82 & 48386 & glycolysis \\
\hline 156 & K3YZ28 & Uncharacterized protein & S. italica & 2 & 90 & 0.11 & 10.59 & 15971 & protein \\
\hline 157 & K3XXR2 & Uncharacterized protein & S. italica & 13 & 117 & 0.11 & 9.20 & 39036 & transport \\
\hline 158 & K3YG24 & Uncharacterized protein & S. italica & 30 & 496 & 0.11 & 7.31 & 107135 & TCA \\
\hline 159 & F2DGF3 & Predicted protein & H. vulgare & 24 & 641 & 0.11 & 6.81 & 115601 & TCA \\
\hline
\end{tabular}




\begin{tabular}{|c|c|c|c|c|c|c|c|c|c|}
\hline 160 & D3GQL1 & Aconitate hydratase 3 & C. clementina & 25 & 310 & 0.11 & 6.30 & 98669 & TCA \\
\hline 161 & B5LAS7 & Putative acetyl co-enzyme A carboxylase biotin carboxylase subunit & C. аппиит & 10 & 125 & 0.11 & 7.06 & 58977 & lipid metabolism \\
\hline 162 & Q0ZII1 & Joka20 & N. plumbaginifolia & 2 & 92 & 0.11 & 8.94 & 17109 & protein \\
\hline 163 & Q8L994 & T-complex protein 1 subunit delta & A. thaliana & 13 & 238 & 0.11 & 7.68 & 58177 & protein \\
\hline 164 & Q4H1G2 & Methionine synthase & B. vulgaris & 28 & 511 & 0.10 & 6.46 & 88546 & amino acid metabolism \\
\hline 165 & E5GBX7 & NADH-ubiquinone oxidoreductase protein & C. melo & 11 & 130 & 0.10 & 8.05 & 53578 & mitoETC \\
\hline 166 & F2D269 & Predicted protein & H. vulgare & 17 & 158 & 0.10 & 5.04 & 73306 & stress \\
\hline 167 & Q08130 & Branching enzyme & M. esculenta & 4 & 28 & 0.10 & 4.70 & 17765 & major $\mathrm{CHO}$ metabolism \\
\hline 168 & B4FUS2 & $40 \mathrm{~S}$ ribosomal protein $\mathrm{S} 18$ & Z. mays & 7 & 48 & 0.10 & 10.74 & 17741 & protein \\
\hline 169 & B6SHD3 & Malate dehydrogenase & Z. mays & 8 & 81 & 0.10 & 8.32 & 42623 & TCA \\
\hline 170 & D6PAY2 & Peptidyl-prolyl cis-trans isomerase & V. hybrid cultivar & 4 & 78 & 0.10 & 8.46 & 18582 & cell \\
\hline 171 & Q8LGJ6 & Phosphoglycerate dehydrogenase-like protein & A. thaliana & 7 & 152 & 0.10 & 6.76 & 63556 & amino acid metabolism \\
\hline 172 & K4A6N0 & Uncharacterized protein & S. italica & 10 & 173 & 0.10 & 7.78 & 75831 & misc \\
\hline 173 & Q0W9E2 & B. chaperonin 60 & S. commersonii & 12 & 340 & 0.10 & 7.27 & 63333 & protein \\
\hline 174 & Q940P8 & T-complex protein 1 subunit beta & A. thaliana & 19 & 336 & 0.09 & 5.87 & 57763 & protein \\
\hline 175 & F2CQ27 & Predicted protein & H. vulgare & 5 & 83 & 0.09 & 6.66 & 25460 & signalling \\
\hline 176 & F2E3I7 & Glutamine synthetase & H. vulgare & 12 & 127 & 0.09 & 5.48 & 39331 & $\mathrm{~N}$-metabolism \\
\hline 177 & K3XW85 & T-complex protein 1 subunit alpha & S. italica & 12 & 359 & 0.09 & 6.09 & 59355 & protein \\
\hline 178 & E4MX73 & mRNA, clone: RTFL01-10-N01 & T. halophila & 4 & 126 & 0.09 & 5.83 & 39684 & TCA \\
\hline 179 & B0M1B1 & Peroxisomal glycolate oxidase & G. $\max$ & 11 & 68 & 0.09 & 9.13 & 40825 & photosynthesis \\
\hline 180 & A9PE52 & Gamma class glutathione transferase EF1Bgamma2 & P. trichocarpa & 10 & 212 & 0.09 & 5.80 & 48127 & protein \\
\hline 181 & A5JVC6 & Putative uncharacterized protein & B. campestris & 9 & 64 & 0.09 & 6.25 & 34259 & cell wall \\
\hline 182 & F2DQ10 & Predicted protein & H. vulgare & 16 & 279 & 0.09 & 5.19 & 98573 & protein \\
\hline 183 & B6UDN1 & Fructose-bisphosphate aldolase & Z. mays & 6 & 189 & 0.09 & 7.96 & 41956 & photosynthesis \\
\hline 184 & Q9C6Z3 & Pyruvate dehydrogenase E1 component subunit beta-2, chloroplastic & A. thaliana & 6 & 95 & 0.09 & 6.35 & 44672 & TCA \\
\hline 185 & B9DH71 & Ketol-acid reductoisomerase & A. thaliana & 8 & 272 & 0.08 & 6.99 & 64142 & amino acid metabolism \\
\hline 186 & B9SGJ6 & Ketol-acid reductoisomerase, chloroplast, putative & R. communis & 9 & 293 & 0.08 & 6.80 & 63712 & amino acid metabolism \\
\hline 187 & Q5NKW4 & Photosystem I reaction center subunit II, $20 \mathrm{kDa}$ & C. reinhardtii & 4 & 55 & 0.08 & 10.30 & 21442 & photosynthesis \\
\hline 188 & A8MRH4 & $60 \mathrm{~S}$ ribosomal protein $\mathrm{L} 7-2$ & A. thaliana & 4 & 40 & 0.08 & 10.10 & 21540 & protein \\
\hline 189 & Q94KB0 & Importin subunit alpha & C. аппиит & 9 & 259 & 0.08 & 5.39 & 59910 & protein \\
\hline 190 & Q9XEN8 & Annexin & N. tabacum & 7 & 150 & 0.08 & 5.53 & 36029 & cell \\
\hline 191 & Q93XK4 & Chlorophyll a/b binding protein & P. contorta & 8 & 113 & 0.08 & 5.59 & 29128 & photosynthesis \\
\hline
\end{tabular}




\begin{tabular}{|c|c|c|c|c|c|c|c|c|c|}
\hline 192 & K3Z566 & Uncharacterized protein & S. italica & 10 & 177 & 0.08 & 7.77 & 59005 & C1-metabolism \\
\hline 193 & G9MA91 & Fructose-bisphosphate aldolase & L. grandiflorum & 5 & 202 & 0.08 & 6.28 & 37690 & glycolysis \\
\hline 194 & B3TM44 & Ribosomal protein S7 & E. guineensis & 2 & 68 & 0.08 & 9.77 & 21923 & protein \\
\hline 195 & Q7XJB0 & Eukaryotic translation initiation factor iso4E & L. sativa & 2 & 154 & 0.08 & 5.69 & 22125 & protein \\
\hline 196 & F2CT86 & Predicted protein & H. vulgare & 2 & 60 & 0.08 & 7.47 & 22104 & signalling \\
\hline 197 & Q645M9 & Glyoxisomal malate dehydrogenase & S. lycopersicum & 8 & 155 & 0.08 & 7.94 & 38007 & gluconeogenesis \\
\hline 198 & B4G1D2 & CBS domain protein & Z. mays & 4 & 56 & 0.08 & 9.33 & 22541 & not assigned \\
\hline 199 & A8MQP6 & Nascent polypeptide-associated complex subunit alpha-like protein 4 & A. thaliana & 4 & 112 & 0.08 & 4.42 & 23100 & protein \\
\hline 200 & Q0MYQ7 & Germin-like protein 2 & V. vinifera & 2 & 122 & 0.08 & 8.41 & 22832 & stress \\
\hline 201 & A9ZMZ5 & Acetyl-CoA C-acetyltransferase & H. brasiliensis & 3 & 117 & 0.07 & 6.68 & 42039 & secondary metabolism \\
\hline 202 & K3YSH1 & Uncharacterized protein & S. italica & 8 & 52 & 0.07 & 9.01 & 48860 & not assigned \\
\hline 203 & E5LCX1 & C14 cysteine protease & S. demissum & 2 & 125 & 0.07 & 4.98 & 23974 & protein \\
\hline 204 & I0CC95 & Type II peroxiredoxin & T. hispida & 2 & 50 & 0.07 & 8.31 & 24226 & redox \\
\hline 205 & Q9M2T8 & 2-oxoglutarate dehydrogenase, E1 subunit-like protein & A. thaliana & 19 & 377 & 0.07 & 7.14 & 114888 & TCA \\
\hline 206 & C8YNG6 & 3-ketoacyl CoA thiolase 1 & P. hybrida & 7 & 127 & 0.07 & 8.29 & 49405 & amino acid metabolism \\
\hline 207 & B3TLP3 & Translation elongation factor EF-1 B. chain & E. guineensis & 10 & 216 & 0.07 & 4.69 & 24523 & protein \\
\hline 208 & B3TLY3 & Ribosomal protein L15 & E. guineensis & 2 & 127 & 0.07 & 11.49 & 24371 & protein \\
\hline 209 & K4A699 & Uncharacterized protein & S. italica & 9 & 437 & 0.07 & 6.16 & 81644 & mitoETC \\
\hline 210 & A8MRE8 & Triosephosphate isomerase & A. thaliana & 5 & 119 & 0.07 & 7.49 & 32458 & photosynthesis \\
\hline 211 & B4FLQ8 & $26 \mathrm{~S}$ proteasome non-ATPase regulatory subunit 14 & Z. mays & 7 & 93 & 0.07 & 6.76 & 34443 & protein \\
\hline 212 & O81609 & Nodule-enhanced malate dehydrogenase & P. sativum & 4 & 173 & 0.07 & 7.75 & 42106 & TCA \\
\hline 213 & K3XVH0 & Uncharacterized protein & S. italica & 9 & 69 & 0.07 & 6.62 & 80203 & photosynthesis \\
\hline 214 & Q6R9J5 & ATPase subunit 4 & Z. mays & 2 & 74 & 0.07 & 8.48 & 24941 & mitoETC \\
\hline 215 & H6VUT7 & Translation elongation factor & A. mongolicus & 12 & 168 & 0.07 & 4.63 & 25300 & protein \\
\hline 216 & K3YKV0 & Uncharacterized protein & S. italica & 18 & 403 & 0.07 & 5.54 & 87462 & cell \\
\hline 217 & B6ZK00 & Peroxisomal biogenesis factor 11 family protein & G. $\max$ & 4 & 93 & 0.07 & 9.76 & 26114 & cell \\
\hline 218 & F2DP49 & Predicted protein & H. vulgare & 5 & 128 & 0.07 & 8.63 & 25913 & hormone metabolism \\
\hline 219 & Q9C6U3 & Putative uncharacterized protein T8G24.2 & A. thaliana & 5 & 135 & 0.07 & 7.59 & 34907 & not assigned \\
\hline 220 & Q9SR15 & Putative tryptophanyl-tRNA synthetase & A. thaliana & 3 & 108 & 0.07 & 5.95 & 46010 & protein \\
\hline 221 & Q93WQ1 & Dihydrolipoyl dehydrogenase & B. gymnorhiza & 8 & 82 & 0.07 & 7.17 & 54389 & TCA \\
\hline 222 & E4MY36 & mRNA, clone: RTFL01-37-B16 & T. halophila & 11 & 86 & 0.06 & 6.25 & 63555 & glycolysis \\
\hline 223 & Q6T379 & Triosephosphate isomerase & S. chacoense & 4 & 31 & 0.06 & 5.99 & 27251 & glycolysis \\
\hline
\end{tabular}




\begin{tabular}{|c|c|c|c|c|c|c|c|c|c|}
\hline 224 & Q8LG45 & Small nuclear ribonucleoprotein-associated protein & A. thaliana & 2 & 77 & 0.06 & 10.71 & 26994 & RNA \\
\hline 225 & Q8LG68 & UDP-glucose 6-dehydrogenase & A. thaliana & 8 & 199 & 0.06 & 6.18 & 53591 & stress \\
\hline 226 & F2E0C0 & Predicted protein & H. vulgare & 13 & 49 & 0.06 & 10.04 & 28326 & protein \\
\hline 227 & Q9MAM6 & Pyruvate dehydrogenase E1 component subunit alpha-3, chloroplastic & A. thaliana & 10 & 45 & 0.06 & 8.76 & 75745 & TCA \\
\hline 228 & Q8LFK9 & 3-dehydroquinate synthase-like protein & A. thaliana & 4 & 118 & 0.06 & 7.15 & 48377 & amino acid metabolism \\
\hline 229 & K4A6V8 & Uncharacterized protein & S. italica & 7 & 149 & 0.06 & 7.03 & 70549 & glycolysis \\
\hline 230 & F6H3T7 & Putative uncharacterized protein & V. vinifera & 15 & 174 & 0.06 & 6.11 & 40651 & not assigned \\
\hline 231 & K3ZS25 & Uncharacterized protein & S. italica & 5 & 145 & 0.06 & 6.39 & 61335 & misc \\
\hline 232 & F2CXX0 & Predicted protein & H. vulgare & 2 & 73 & 0.06 & 5.50 & 30839 & photosynthesis \\
\hline 233 & Q2V4Q4 & 50S ribosomal protein $\mathrm{L} 4$ & A. thaliana & 3 & 77 & 0.06 & 9.26 & 30124 & protein \\
\hline 234 & F2D319 & Citrate synthase & H. vulgare & 3 & 125 & 0.06 & 7.24 & 40252 & gluconeogenesis \\
\hline 235 & O04998 & Glutamine synthetase & M. truncatula & 5 & 59 & 0.06 & 5.73 & 39326 & $\mathrm{~N}$-metabolism \\
\hline 236 & B6U0V6 & Endoplasmin & Z. mays & 17 & 367 & 0.06 & 4.97 & 92831 & stress \\
\hline 237 & F2DAP7 & Malic enzyme & H. vulgare & 8 & 122 & 0.06 & 5.78 & 69436 & TCA \\
\hline 238 & E4MVN2 & mRNA, clone: RTFL01-06-F21 & T. halophila & 4 & 83 & 0.06 & 6.42 & 31122 & photosynthesis \\
\hline 239 & Q1I0X5 & Pyruvate kinase & C. аппиит & 8 & 40 & 0.05 & 6.84 & 56114 & glycolysis \\
\hline 240 & Q5GI04 & Hypersensitive-induced reaction protein & C. аппиит & 4 & 89 & 0.05 & 6.42 & 31614 & not assigned \\
\hline 241 & B6TLT1 & Serine/threonine-protein phosphatase $2 \mathrm{~A} 65 \mathrm{kDa}$ regulatory subunit $\mathrm{AB}$. isoform & Z. mays & 12 & 136 & 0.05 & 5.03 & 66318 & protein \\
\hline 242 & K4AAQ4 & Uncharacterized protein & S. italica & 7 & 204 & 0.05 & 10.56 & 44399 & protein \\
\hline 243 & G1JSJ5 & Atlg59610 & A. thaliana & 9 & 202 & 0.05 & 9.04 & 100452 & misc \\
\hline 244 & F2Z9R3 & Glucose-6-phosphate 1-dehydrogenase & N. benthamiana & 9 & 159 & 0.05 & 8.24 & 67846 & OPP \\
\hline 245 & I0J3D8 & Proteasome subunit B. type & A. halleri & 2 & 133 & 0.05 & 6.06 & 33367 & protein \\
\hline 246 & K4A6E4 & Uncharacterized protein & S. italica & 6 & 76 & 0.05 & 5.16 & 83215 & protein \\
\hline 247 & E5RDE1 & $26 \mathrm{~S}$ proteasome non-ATPase regulatory subunit & C. melo & 4 & 113 & 0.05 & 4.73 & 34256 & protein \\
\hline 248 & A8MRF3 & $60 \mathrm{~S}$ ribosomal protein $\mathrm{L} 18 \mathrm{a}-1$ & A. thaliana & 3 & 44 & 0.05 & 9.73 & 36337 & misc \\
\hline 249 & Q38766 & Victorin binding protein & A. sativa & 7 & 69 & 0.05 & 6.96 & 112298 & photosynthesis \\
\hline 250 & K3XVN4 & Uncharacterized protein & S. italica & 5 & 113 & 0.05 & 5.72 & 72670 & protein \\
\hline 251 & K3XJZ1 & Uncharacterized protein & S. italica & 2 & 51 & 0.05 & 9.39 & 35932 & protein \\
\hline 252 & K3XY79 & Uncharacterized protein & S. italica & 3 & 42 & 0.05 & 5.22 & 37046 & secondary metabolism \\
\hline 253 & А9РВТ8 & Putative uncharacterized protein & P. trichocarpa & 4 & 78 & 0.05 & 6.67 & 48985 & protein \\
\hline 254 & K4A5J5 & Uncharacterized protein & S. italica & 10 & 259 & 0.05 & 5.08 & 100819 & cell \\
\hline 255 & K3ZTJ3 & Uncharacterized protein & S. italica & 7 & 90 & 0.05 & 8.94 & 47124 & photosynthesis \\
\hline
\end{tabular}




\begin{tabular}{|c|c|c|c|c|c|c|c|c|c|}
\hline 256 & B9HJ23 & Predicted protein & P. trichocarpa & 9 & 158 & 0.05 & 6.54 & 101700 & misc \\
\hline 257 & Q39986 & Cysteine proteinase & Hemerocallis & 3 & 98 & 0.04 & 5.50 & 40006 & protein \\
\hline 258 & E4MVJ5 & mRNA, clone: RTFL01-06-D09 & T. halophila & 3 & 50 & 0.04 & 8.91 & 40486 & not assigned \\
\hline 259 & Q9ZNX1 & NAD-dependent isocitrate dehydrogenase & N. tabacum & 3 & 98 & 0.04 & 7.52 & 40651 & TCA \\
\hline 260 & Q2LFC3 & AGO1-2 & N. benthamiana & 8 & 201 & 0.04 & 9.31 & 109989 & development \\
\hline 261 & K4A4Z8 & Uncharacterized protein & S. italica & 14 & 193 & 0.04 & 6.96 & 137642 & cell \\
\hline 262 & B6UBQ9 & Ankyrin protein kinase-like & Z. mays & 11 & 178 & 0.04 & 9.03 & 68790 & stress \\
\hline 263 & B4FYM0 & Acetyl-CoA acetyltransferase, cytosolic 2 & Z. mays & 4 & 147 & 0.04 & 7.66 & 43450 & secondary metabolism \\
\hline 264 & E1ZPL7 & Putative uncharacterized protein & C. variabilis & 2 & 71 & 0.04 & 8.88 & 41498 & signalling \\
\hline 265 & P93698 & Lipoxygenase & V. unguiculata & 7 & 190 & 0.04 & 6.49 & 102731 & hormone metabolism \\
\hline 266 & A9NUN5 & Putative uncharacterized protein & P. sitchensis & 3 & 90 & 0.04 & 8.81 & 45137 & amino acid metabolism \\
\hline 267 & F2DLJ7 & Predicted protein & H. vulgare & 2 & 112 & 0.04 & 7.77 & 44423 & hormone metabolism \\
\hline 268 & A9P9A2 & Putative uncharacterized protein & P. trichocarpa & 10 & 109 & 0.04 & 9.36 & 46272 & RNA \\
\hline 269 & K3XE87 & Uncharacterized protein & S. italica & 7 & 139 & 0.04 & 5.90 & 106127 & cell \\
\hline 270 & Q9SMC2 & Acetolactate synthase small subunit & N. plumbaginifolia & 5 & 102 & 0.03 & 7.27 & 50423 & amino acid metabolism \\
\hline 271 & F2DY57 & Predicted protein & H. vulgare & 3 & 66 & 0.03 & 5.95 & 50880 & glycolysis \\
\hline 272 & I1LJS6 & Lipoxygenase & G. $\max$ & 4 & 107 & 0.03 & 7.36 & 104140 & hormone metabolism \\
\hline 273 & C5WQZ5 & Putative uncharacterized protein Sb01g041210 & S. bicolor & 3 & 155 & 0.03 & 7.65 & 51802 & protein \\
\hline 274 & K4A9K9 & Uncharacterized protein & S. italica & 2 & 41 & 0.03 & 5.83 & 52168 & secondary metabolism \\
\hline 275 & K3YHL2 & Uncharacterized protein & S. italica & 6 & 85 & 0.03 & 9.42 & 48818 & stress \\
\hline 276 & K3ZSX0 & Uncharacterized protein & S. italica & 8 & 156 & 0.03 & 7.64 & 53564 & amino acid metabolism \\
\hline 277 & Q9S768 & Alanine aminotransferase & O. sativa & 2 & 195 & 0.03 & 6.65 & 53130 & amino acid metabolism \\
\hline 278 & Q9FY49 & Leukotriene-A4 hydrolase & A. thaliana & 4 & 67 & 0.03 & 5.24 & 69621 & protein \\
\hline 279 & F2DIS4 & Malic enzyme & H. vulgare & 6 & 99 & 0.03 & 7.40 & 68411 & TCA \\
\hline 280 & B2KNE6 & Phospholipase D & H. аппии & 10 & 189 & 0.03 & 5.52 & 92315 & lipid metabolism \\
\hline 281 & K3XWI6 & Uncharacterized protein & S. italica & 8 & 210 & 0.03 & 6.60 & 55489 & glycolysis \\
\hline 282 & Q9SZ27 & Putative aspartate-tRNA ligase & A. thaliana & 2 & 128 & 0.03 & 6.37 & 57768 & protein \\
\hline 283 & Q9SPB1 & Leghemoglobin reductase & V. unguiculata & 5 & 62 & 0.03 & 8.05 & 56030 & TCA \\
\hline 284 & K3YH48 & Uncharacterized protein & S. italica & 5 & 56 & 0.03 & 8.66 & 56052 & TCA \\
\hline 285 & K3YGV5 & Uncharacterized protein & S. italica & 4 & 73 & 0.03 & 8.10 & 62283 & amino acid metabolism \\
\hline 286 & Q4ZJ74 & Allene oxide synthase & H. brasiliensis & 2 & 100 & 0.03 & 8.65 & 58783 & hormone metabolism \\
\hline 287 & Q41444 & Mitochondrial processing peptidase & S. tuberosum & 5 & 110 & 0.03 & 6.64 & 59463 & protein \\
\hline
\end{tabular}




\begin{tabular}{|c|c|c|c|c|c|c|c|c|c|}
\hline 288 & Q41445 & Mitochondrial processing peptidase & S. tuberosum & 2 & 37 & 0.03 & 6.73 & 60107 & protein \\
\hline 289 & Q9M084 & AT4G31180 protein & A. thaliana & 3 & 148 & 0.03 & 6.23 & 63391 & protein \\
\hline 290 & E1Z5Z1 & Putative uncharacterized protein & C. variabilis & 7 & 64 & 0.03 & 7.27 & 63288 & RNA \\
\hline 291 & B9ICC7 & Calnexin family protein & P. trichocarpa & 5 & 71 & 0.03 & 4.78 & 60802 & signalling \\
\hline 292 & O78327 & Transketolase 1 & С. аппиит & 4 & 89 & 0.03 & 6.62 & 80398 & photosynthesis \\
\hline 293 & Q4LDR0 & Heat shock protein & S. lycopersicum & 7 & 120 & 0.02 & 6.55 & 110564 & stress \\
\hline 294 & F2DN74 & Methionine--tRNA ligase & H. vulgare & 2 & 64 & 0.02 & 7.01 & 72371 & protein \\
\hline 295 & A9PII8 & Putative uncharacterized protein & P. trichocarpa & 5 & 61 & 0.02 & 8.29 & 67342 & redox \\
\hline 296 & A8IBE0 & Predicted protein & C. reinhardtii & 2 & 26 & 0.02 & 9.22 & 75068 & RNA \\
\hline 297 & Q42793 & Acetyl CoA carboxylase & G. $\max$ & 18 & 182 & 0.02 & 6.32 & 253225 & lipid metabolism \\
\hline 298 & Q2LFC1 & AGO4-2 & N. benthamiana & 8 & 40 & 0.02 & 8.78 & 102331 & RNA \\
\hline 299 & Q8L6J9 & Putative carbamoyl phosphate synthase large subunit & N. tabacum & 7 & 98 & 0.02 & 5.91 & 133241 & nucleotide metabolism \\
\hline 300 & B0FNB2 & Pyrroline-5-carboxylate synthetase & P. vulgaris & 4 & 47 & 0.02 & 6.62 & 78099 & amino acid metabolism \\
\hline 301 & F6H740 & Putative uncharacterized protein & V. vinifera & 2 & 31 & 0.02 & 7.99 & 84234 & cell wall \\
\hline 302 & Q5CCG4 & Acetyl-CoA carboxylase & A. myosuroides & 4 & 109 & 0.02 & 5.87 & 82215 & lipid metabolism \\
\hline 303 & G5DWG4 & DEAD-box ATP-dependent RNA helicase & S. latifolia & 5 & 28 & 0.02 & 6.02 & 85314 & RNA \\
\hline 304 & E5GBL0 & Short-chain dehydrogenase/reductase & C. melo & 4 & 38 & 0.02 & 6.89 & 108844 & RNA \\
\hline 305 & B5LAU8 & Putative ferredoxin-dependent glutamate synthase 1 & C. аппиит & 13 & 182 & 0.02 & 6.54 & 179205 & $\mathrm{~N}$-metabolism \\
\hline 306 & K3Z3P5 & Uncharacterized protein & S. italica & 11 & 98 & 0.02 & 6.25 & 95578 & protein \\
\hline 307 & B9HRI3 & V-type proton ATPase subunit a & P. trichocarpa & 6 & 86 & 0.02 & 6.10 & 93462 & transport \\
\hline 308 & Q40360 & NADH-dependent glutamate synthase & M. sativa & 12 & 81 & 0.02 & 6.27 & 242513 & $\mathrm{~N}$-metabolism \\
\hline 309 & K3XE63 & Uncharacterized protein & S. italica & 4 & 90 & 0.02 & 6.04 & 108302 & cell \\
\hline 310 & Q9SQH4 & Actin bundling protein $\mathrm{ABP} 135$ & L. longiflorum & 5 & 38 & 0.02 & 5.97 & 107053 & cell \\
\hline 311 & F2DKP3 & Beta-adaptin-like protein & H. vulgare & 2 & 39 & 0.02 & 5.10 & 99927 & cell \\
\hline 312 & Q8L5C2 & $110 \mathrm{kDa} 4 \mathrm{SNc}$-Tudor domain protein & P. sativum & 7 & 39 & 0.02 & 7.36 & 108600 & RNA \\
\hline 313 & Q9LY69 & Putative uncharacterized protein MAA21_90 & A. thaliana & 2 & 49 & 0.01 & 5.11 & 119971 & not assigned \\
\hline 314 & F2CRM6 & Predicted protein & H. vulgare & 2 & 163 & 0.01 & 5.52 & 121394 & protein \\
\hline 315 & Q9LY25 & Putative uncharacterized protein T2I1_60 & A. thaliana & 10 & 43 & 0.01 & 6.40 & 115126 & RNA \\
\hline 316 & Q9FVU8 & Putative UDP-glucose:glycoprotein glucosyltransferase; 101200-91134 & A. thaliana & 7 & 104 & 0.01 & 5.77 & 189722 & protein \\
\hline 317 & E1ZQ18 & Putative uncharacterized protein & C. variabilis & 4 & 26 & 0.01 & 5.39 & 183606 & cell \\
\hline
\end{tabular}

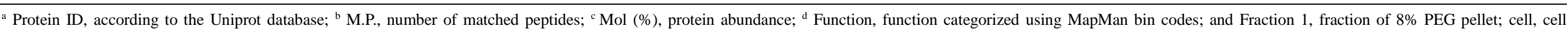

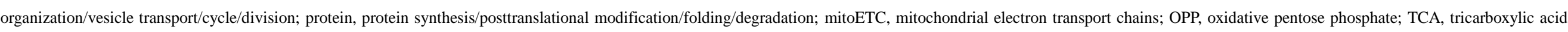
cycle; redox, redox ascorbate/glutathione metabolism; RNA, RNA processing/regulation of transcription; and misc, miscellaneous. 
Supplemental Table 8. Proteins Identified in L. japonica Immature Flower Buds at Fraction 2 using Polyethylene Glycol Fractionation.

\begin{tabular}{|c|c|c|c|c|c|c|c|c|c|}
\hline No. & Protein ID ${ }^{\text {a }}$ & Description & Species & M.P. ${ }^{\mathrm{b}}$ & Score & $\operatorname{Mol}(\%)^{\mathrm{c}}$ & $\mathrm{pI}$ & Mass (Da) & Function $^{\mathrm{d}}$ \\
\hline 1 & C3S8R0 & Ribulose-1,5-bisphosphate carboxylase/oxygenase large subunit & L. gracilis & 41 & 259 & 1.50 & 6.92 & 47818 & photosynthesis \\
\hline 2 & Q8MCW6 & Ribulose-1,5-bisphosphate carboxylase large subunit & M. racemosa & 43 & 349 & 1.42 & 6.58 & 51631 & photosynthesis \\
\hline 3 & B8PRL9 & Ribulose bisphosphate carboxylase large chain & A. canadensis & 39 & 278 & 1.37 & 5.62 & 52414 & photosynthesis \\
\hline 4 & F2VXU4 & Arf2 & H. brasiliensis & 8 & 205 & 1.27 & 6.43 & 20724 & protein \\
\hline 5 & Q8MCY5 & Ribulose bisphosphate carboxylase large chain & T. cymosum & 28 & 210 & 1.17 & 7.56 & 44221 & photosynthesis \\
\hline 6 & E0D6S0 & Actin & G. bicolor & 23 & 395 & 1.00 & 5.31 & 41913 & cell \\
\hline 7 & B5M4B1 & Beta-tubulin & S. tuberosum & 16 & 270 & 0.85 & 4.86 & 51166 & cell \\
\hline 8 & J9QAL9 & Ribosomal protein L2 & A. corniculatum & 4 & 90 & 0.81 & 10.23 & 21703 & protein \\
\hline 9 & K3ZA95 & Ribulose bisphosphate carboxylase small chain & S. italica & 9 & 59 & 0.76 & 8.53 & 19471 & photosynthesis \\
\hline 10 & C6GFP3 & ATP synthase subunit beta & G. hirsutum & 16 & 380 & 0.73 & 6.29 & 59987 & mitoETC \\
\hline 11 & G9FCM9 & ATP synthase subunit alpha & B. hygrometrica & 27 & 242 & 0.65 & 6.61 & 55364 & mitoETC \\
\hline 12 & Q5S817 & ATP synthase subunit alpha & H. madagascariensis & 33 & 553 & 0.65 & 6.3 & 46438 & mitoETC \\
\hline 13 & K3ZDK9 & Uncharacterized protein & S. italica & 2 & 112 & 0.57 & 5.05 & 15191 & protein \\
\hline 14 & B9H2U7 & Predicted protein & P. trichocarpa & 7 & 191 & 0.52 & 7.28 & 64204 & amino acid metabolism \\
\hline 15 & A2Y926 & Proteasome subunit beta type & O. sativa & 5 & 75 & 0.50 & 5.48 & 26357 & protein \\
\hline 16 & Q9FEM4 & Catalase & B. pendula & 5 & 99 & 0.46 & 5.48 & 17247 & redox \\
\hline 17 & Q9AVU8 & Putative vacuolar ATP Synthase subunit A & M. crystallinum & 13 & 314 & 0.46 & 5.21 & 69139 & transport \\
\hline 18 & H9NIE1 & Cell division cycle protein 48 & C. sinensis & 22 & 316 & 0.41 & 5.29 & 90644 & cell \\
\hline 19 & B6EBD7 & Heat shock protein $90-1$ & G. $\max$ & 22 & 194 & 0.40 & 5.02 & 80700 & stress \\
\hline 20 & E4MVY3 & mRNA, clone: RTFL01-01-M18 & T. halophila & 20 & 257 & 0.40 & 6.25 & 94734 & protein \\
\hline 21 & Q43106 & $\mathrm{H}(+)$-transporting ATPase & P. vulgaris & 16 & 262 & 0.39 & 7.21 & 104776 & transport \\
\hline 22 & F2DLC2 & Proteasome subunit alpha type & H. vulgare & 10 & 313 & 0.38 & 4.68 & 26087 & protein \\
\hline 23 & G9FCP1 & $\mathrm{NADH}$-quinone oxidoreductase subunit $\mathrm{D}$ & B. hygrometrica & 4 & 76 & 0.38 & 7.56 & 44776 & mitoETC \\
\hline 24 & $\mathrm{~A} 2 \mathrm{~V} 880$ & $\mathrm{G}$ protein beta-subunit-like protein & N. tabacum & 3 & 127 & 0.37 & 7.09 & 16100 & signalling \\
\hline 25 & Q9SC26 & ATP synthase subunit beta & S. californica & 10 & 229 & 0.37 & 5.24 & 53636 & photosynthesis \\
\hline 26 & K3XFR6 & Uncharacterized protein & S. italica & 10 & 163 & 0.36 & 7.46 & 66448 & TCA \\
\hline 27 & Q645M9 & Glyoxisomal malate dehydrogenase & S. lycopersicum & 6 & 241 & 0.34 & 7.94 & 38007 & gluconeogenesis \\
\hline 28 & Q9C5M0 & Mitochondrial dicarboxylate/tricarboxylate transporter DTC & A. thaliana & 6 & 70 & 0.33 & 9.23 & 32347 & transport \\
\hline 29 & A3QQY2 & Sucrose synthase & C. intybus & 11 & 92 & 0.33 & 6.39 & 92338 & major $\mathrm{CHO}$ metabolism \\
\hline 30 & Q42912 & Serine/threonine-protein phosphatase & M. domestica & 2 & 94 & 0.33 & 5.01 & 35419 & protein \\
\hline 31 & K3XLU5 & Uncharacterized protein & S. italica & 4 & 62 & 0.31 & 10.08 & 24413 & protein \\
\hline
\end{tabular}




\begin{tabular}{|c|c|c|c|c|c|c|c|c|c|}
\hline 32 & B6ZK00 & Peroxisomal biogenesis factor 11 family protein & G. $\max$ & 2 & 72 & 0.30 & 9.76 & 26114 & cell \\
\hline 33 & K3ZWL4 & Uncharacterized protein & S. italica & 3 & 79 & 0.30 & 9.66 & 26013 & protein \\
\hline 34 & K3YR06 & Uncharacterized protein & S. italica & 5 & 220 & 0.29 & 6.16 & 64225 & protein \\
\hline 35 & Q9ZNX1 & NAD-dependent isocitrate dehydrogenase & N. tabacum & 5 & 82 & 0.28 & 7.52 & 40651 & TCA \\
\hline 36 & E5GBL8 & ATP-dependent clp protease & C. melo & 18 & 173 & 0.27 & 7.23 & 103416 & protein \\
\hline 37 & F2D9E0 & Predicted protein & H. vulgare & 3 & 102 & 0.27 & 11.14 & 28037 & protein \\
\hline 38 & K3Z5F7 & Uncharacterized protein & S. italica & 3 & 120 & 0.27 & 6.67 & 58832 & protein \\
\hline 39 & K017G7 & Thioredoxin peroxidase & N. tabacum & 7 & 89 & 0.25 & 8.03 & 29972 & redox \\
\hline 40 & Q4W5U7 & Calnexin-like protein & S. lycopersicum & 5 & 88 & 0.25 & 4.82 & 61338 & signalling \\
\hline 41 & K4A7I4 & Uncharacterized protein & S. italica & 12 & 343 & 0.25 & 5.4 & 61666 & photosynthesis \\
\hline 42 & Q8H945 & Phosphoenolpyruvate carboxylase & L. japonicus & 9 & 150 & 0.25 & 5.88 & 111020 & glycolysis \\
\hline 43 & I0J3D8 & Proteasome subunit beta type & A. halleri & 4 & 113 & 0.24 & 6.06 & 33367 & protein \\
\hline 44 & C6T859 & Beta-form rubisco activase & G. $\max$ & 9 & 216 & 0.24 & 7.24 & 48764 & photosynthesis \\
\hline 45 & F2DXI0 & Predicted protein & H. vulgare & 14 & 134 & 0.22 & 7.09 & 102090 & protein \\
\hline 46 & D7NHW9 & 2-phospho-D-glycerate hydrolase & P. trifoliata & 5 & 69 & 0.22 & 5.78 & 47986 & glycolysis \\
\hline 47 & C9WSP8 & Plasma membrane intrinsic protein & H. brasiliensis & 2 & 167 & 0.18 & 7.74 & 30978 & transport \\
\hline 48 & K3ZUF9 & Uncharacterized protein & S. italica & 8 & 108 & 0.18 & 8.6 & 41440 & signalling \\
\hline 49 & Q1I0X5 & Pyruvate kinase & C. аппиит & 4 & 118 & 0.17 & 6.84 & 56114 & glycolysis \\
\hline 50 & Q5GAQ3 & Putative alanine aminotransferase & Z. mays & 3 & 98 & 0.17 & 5.64 & 57003 & amino acid metabolism \\
\hline 51 & E4MXI2 & mRNA, clone: RTFL01-39-D20 & T. halophila & 7 & 93 & 0.17 & 5.21 & 73890 & stress \\
\hline 52 & C5X0Q9 & Sucrose synthase & S. bicolor & 7 & 44 & 0.16 & 6.86 & 92627 & major $\mathrm{CHO}$ metabolism \\
\hline 53 & F2E797 & Predicted protein & H. vulgare & 7 & 127 & 0.16 & 5.72 & 47111 & TCA \\
\hline 54 & Q7YAM3 & NADH dehydrogenase subunit 7 & C. vulgaris & 2 & 89 & 0.16 & 6.98 & 45253 & mitoETC \\
\hline 55 & K4A5J5 & Uncharacterized protein & S. italica & 4 & 98 & 0.16 & 5.08 & 100819 & cell \\
\hline 56 & Q9SK84 & Class-II DAHP synthetase-like protein & A. thaliana & 11 & 114 & 0.16 & 8.32 & 58689 & amino acid metabolism \\
\hline 57 & K4A6V8 & Uncharacterized protein & S. italica & 3 & 128 & 0.15 & 7.03 & 70549 & glycolysis \\
\hline 58 & B3H778 & Argininosuccinate synthase & A. thaliana & 4 & 52 & 0.15 & 7.72 & 49340 & amino acid metabolism \\
\hline 59 & F2CRZ5 & Predicted protein & H. vulgare & 3 & 52 & 0.15 & 5.52 & 49253 & protein \\
\hline 60 & K3YHH7 & Uncharacterized protein & S. italica & 3 & 76 & 0.15 & 8.72 & 51287 & protein \\
\hline 61 & Q8LCK7 & Putative uncharacterized protein & A. thaliana & 5 & 82 & 0.15 & 7.56 & 48349 & not assigned \\
\hline 62 & Q9SUU0 & Serine hydroxymethyltransferase & A. thaliana & 4 & 161 & 0.15 & 8.05 & 50988 & photosynthesis \\
\hline 63 & Q0W9E2 & Beta chaperonin 60 & S. commersonii & 7 & 95 & 0.15 & 7.27 & 63333 & protein \\
\hline
\end{tabular}




\begin{tabular}{|c|c|c|c|c|c|c|c|c|c|}
\hline 64 & K3ZQC3 & Uncharacterized protein & S. italica & 5 & 111 & 0.14 & 7.23 & 115569 & TCA \\
\hline 65 & F2CSM1 & Predicted protein & H. vulgare & 6 & 46 & 0.13 & 6.84 & 55969 & protein \\
\hline 66 & Q9SZ27 & Putative aspartate-tRNA ligase & A. thaliana & 2 & 96 & 0.13 & 6.37 & 57768 & protein \\
\hline 67 & Q39834 & Clathrin heavy chain & G. $\max$ & 13 & 255 & 0.12 & 5.55 & 194430 & cell \\
\hline 68 & K3Y4M8 & Uncharacterized protein & S. italica & 7 & 199 & 0.10 & 5.39 & 194893 & cell \\
\hline 69 & Q30DX9 & 2-isopropylmalate synthase & B. insularis & 2 & 99 & 0.08 & 6.44 & 68109 & amino acid metabolism \\
\hline 70 & Q38766 & Victorin binding protein & A. sativa & 6 & 128 & 0.08 & 6.96 & 112298 & photosynthesis \\
\hline 71 & Q4LDR0 & Heat shock protein & S. lycopersicum & 8 & 137 & 0.08 & 6.55 & 110564 & stress \\
\hline 72 & Q9XFX7 & Phospholipase D & C. plantagineum & 5 & 103 & 0.07 & 5.66 & 92228 & lipid metabolism \\
\hline 73 & K3ZQ66 & Uncharacterized protein & S. italica & 9 & 51 & 0.06 & 4.92 & 124515 & not assigned \\
\hline
\end{tabular}

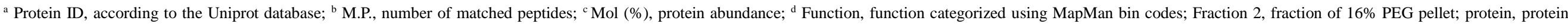
synthesis/folding/degradation; cell, cell organization/vesicle transport/cycle/division; mitoETC, mitochondrial electron transport chains; redox, redox ascorbate/glutathione metabolism; and TCA, tricarboxylic acid cycle;. 
Supplemental Table 9. Proteins Identified in L. japonica Immature Flower Buds at Fraction 3 using Polyethylene Glycol Fractionation.

\begin{tabular}{|c|c|c|c|c|c|c|c|c|c|}
\hline No. & Protein ID ${ }^{\text {a }}$ & Description & Species & M.P. ${ }^{b}$ & Score & $\operatorname{Mol}(\%)^{\mathrm{c}}$ & $\mathrm{pI}$ & Mass (Da) & Function $^{\mathrm{d}}$ \\
\hline 1 & O81976 & 14-3-3 Protein & G. $\max$ & 56 & 1109 & 2.45 & 4.21 & 7619 & signalling \\
\hline 2 & Q9ZQT0 & Actin & G. echinata & 46 & 468 & 1.85 & 6.19 & 17082 & cell \\
\hline 3 & G0XNX8 & Actin & H. brasiliensis & 111 & 1792 & 1.46 & 5.49 & 41897 & cell \\
\hline 4 & Q41534 & ATP synthase subunit B. & T. aestivum & 125 & 2262 & 1.33 & 5.86 & 59326 & mitoETC \\
\hline 5 & C6GFP3 & ATP synthase subunit B. & G. hirsutum & 157 & 2631 & 1.18 & 6.29 & 59987 & mitoETC \\
\hline 6 & Q8L4S4 & Phosphoglycerate kinase & A. speltoides & 54 & 486 & 0.91 & 5.01 & 31433 & photosynthesis \\
\hline 7 & Q944T3 & Glyceraldehyde 3-phosphate dehydrogenase 2 & F. ananassa & 17 & 414 & 0.89 & 9.2 & 13954 & glycolysis \\
\hline 8 & Q75ZD6 & 14-3-3 h-1 protein & N. tabacum & 88 & 1312 & 0.86 & 4.78 & 29121 & signalling \\
\hline 9 & F2D884 & Predicted protein & H. vulgare & 112 & 1862 & 0.86 & 5.19 & 71476 & stress \\
\hline 10 & Q75ZE0 & 14-3-3 e-1 protein & N. tabacum & 75 & 872 & 0.85 & 4.78 & 29479 & signalling \\
\hline 11 & O65165 & 14-3-3 like protein & M. crystalL. & 2 & 162 & 0.84 & 4.26 & 4502 & signalling \\
\hline 12 & F8WL60 & B. actin & R. stylosa & 59 & 902 & 0.81 & 5.27 & 33424 & cell \\
\hline 13 & C0KZ35 & Glyceraldehyde-3-phosphate dehydrogenase & S. arboricola & 35 & 709 & 0.74 & 7.28 & 21225 & glycolysis \\
\hline 14 & Q40463 & NTGB2 & N. tabacum & 11 & 235 & 0.74 & 6.19 & 15827 & signalling \\
\hline 15 & $\mathrm{C} 8 \mathrm{CP} 48$ & Ubiquitin-conjugating enzyme variant & C. sinensis & 10 & 423 & 0.69 & 6.68 & 16734 & protein \\
\hline 16 & P93785 & 14-3-3 protein & S. tuberosum & 74 & 1304 & 0.67 & 4.82 & 29014 & signalling \\
\hline 17 & I4DSV6 & 14-3-3 protein & M. polymorpha & 73 & 1074 & 0.65 & 4.69 & 29526 & signalling \\
\hline 18 & Q4H1G1 & Adenosylhomocysteinase & B. vulgaris & 80 & 1413 & 0.63 & 6.19 & 54052 & amino acid metabolism \\
\hline 19 & A9P8Q7 & Predicted protein & P. trichocarpa & 62 & 802 & 0.60 & 4.84 & 28824 & signalling \\
\hline 20 & G1DVA7 & Non-specific lipid-transfer protein & H. annuиs & 4 & 43 & 0.60 & 8.41 & 12193 & lipid metabolism \\
\hline 21 & F2DJB5 & Predicted protein & H. vulgare & 17 & 224 & 0.59 & 9.32 & 12285 & mitoETC \\
\hline 22 & Q08II7 & Heat shock protein 70 -like protein & L. sativa & 10 & 220 & 0.58 & 10.33 & 12539 & stress \\
\hline 23 & F2CYA6 & Predicted protein & H. vulgare & 66 & 826 & 0.57 & 6.06 & 17119 & protein \\
\hline 24 & G5DVX2 & Phosphoglycerate kinase & S. latifolia & 72 & 1178 & 0.55 & 6.58 & 51352 & photosynthesis \\
\hline 25 & F2D4W6 & Malate dehydrogenase & H. vulgare & 82 & 1458 & 0.54 & 5.88 & 35920 & TCA \\
\hline 26 & K4ACE3 & Uncharacterized protein & S. italica & 83 & 1374 & 0.54 & 6.09 & 35803 & TCA \\
\hline 27 & D7NHW9 & 2-phospho-D-glycerate hydrolase & P. trifoliata & 63 & 1532 & 0.53 & 5.78 & 47986 & glycolysis \\
\hline 28 & D9ZBV9 & Malate dehydrogenase & S. globosa & 18 & 286 & 0.51 & 4.92 & 13766 & TCA \\
\hline 29 & F8WQS4 & Quinone reductase & N. benthamiana & 13 & 145 & 0.50 & 6.18 & 21229 & lipid metabolism \\
\hline 30 & F2CRF1 & Predicted protein & H. vulgare & 78 & 796 & 0.48 & 4.88 & 29361 & signalling \\
\hline 32 & F2CT86 & Predicted protein & H. vulgare & 13 & 238 & 0.47 & 7.47 & 22104 & signalling \\
\hline
\end{tabular}




\begin{tabular}{|c|c|c|c|c|c|c|c|c|c|}
\hline 31 & Q6PKU2 & Putative glyceraldehyde-3-phosphate dehydrogenase & O. minor & 25 & 351 & 0.47 & 7.09 & 22122 & glycolysis \\
\hline 33 & O81830 & Putative uncharacterized protein AT4g27270 & A. thaliana & 16 & 236 & 0.47 & 6.79 & 22355 & lipid metabolism \\
\hline 34 & Q41340 & Small GTP-binding protein & S. lycopersicum & 12 & 192 & 0.46 & 5.44 & 22775 & signalling \\
\hline 35 & B2VQE0 & Methionine synthase & O. ramosa & 190 & 2182 & 0.45 & 6.4 & 84862 & amino acid metabolism \\
\hline 36 & Q19TV8 & UDP-glucose pyrophosphorylase & C. melo & 28 & 319 & 0.45 & 7.17 & 52201 & glycolysis \\
\hline 37 & D0ELH5 & Peptidyl-prolyl cis-trans isomerase & C. sinensis & 16 & 324 & 0.44 & 8.46 & 18310 & cell \\
\hline 38 & G8GJ68 & Adenosylhomocysteinase & L. usitatissimum & 71 & 1209 & 0.43 & 5.99 & 61872 & amino acid metabolism \\
\hline 39 & B4FB55 & Ras-related protein ARA-3 & Z. mays & 12 & 144 & 0.42 & 8.22 & 24038 & signalling \\
\hline 40 & O04428 & Lactoylglutathione lyase & C. paradisi & 26 & 251 & 0.42 & 5.59 & 32737 & biodegradation of xenobiotics \\
\hline 41 & K4A6U5 & Uncharacterized protein & S. italica & 51 & 854 & 0.41 & 5.34 & 71710 & stress \\
\hline 42 & B3TLL4 & Triosephosphate isomerase & E. guineensis & 22 & 87 & 0.41 & 6.23 & 27476 & glycolysis \\
\hline 43 & Q9M6B3 & Malate dehydrogenase & $V$. vinifera & 57 & 1753 & 0.39 & 8.62 & 37137 & TCA \\
\hline 44 & B6SIC6 & Ubiquitin-conjugating enzyme E2 N & Z. mays & 16 & 200 & 0.38 & 7.34 & 17234 & protein \\
\hline 45 & B3TLW5 & Ubiquitin-conjugating enzyme 1 & E. guineensis & 15 & 210 & 0.38 & 6.57 & 17280 & protein \\
\hline 46 & Q6UNT4 & Putative GTP-binding protein & C. sativus & 14 & 59 & 0.38 & 5.19 & 23366 & signalling \\
\hline 47 & G9M8Q6 & S-adenosylmethionine synthase & L. grandiflorum & 41 & 409 & 0.37 & 8.53 & 36539 & amino acid metabolism \\
\hline 48 & K3XL57 & Uncharacterized protein & S. italica & 19 & 100 & 0.36 & 5.48 & 27435 & glycolysis \\
\hline 49 & B4FL17 & Translationally-controlled tumor protein & Z. mays & 18 & 176 & 0.34 & 4.67 & 18773 & development \\
\hline 50 & G1FB60 & Cytochrome b559 subunit alpha & S. polyrrhiza & 7 & 137 & 0.33 & 4.83 & 9408 & photosynthesis \\
\hline 51 & G5DXK7 & Membrane steroid-binding protein & S. latifolia & 6 & 136 & 0.33 & 4.61 & 23493 & redox \\
\hline 52 & Q6T379 & Triosephosphate isomerase & S. chacoense & 19 & 374 & 0.32 & 5.99 & 27251 & glycolysis \\
\hline 53 & В6Т3Р9 & Enolase & Z. mays & 42 & 867 & 0.31 & 5.82 & 48386 & glycolysis \\
\hline 54 & B6TCZ3 & Malate dehydrogenase & Z. mays & 24 & 514 & 0.31 & 7.74 & 41394 & TCA \\
\hline 55 & A1Z3W7 & eIF5A & R. chinensis & 6 & 156 & 0.31 & 5.99 & 17703 & protein \\
\hline 56 & K3ZXL7 & Uncharacterized protein & S. italica & 12 & 196 & 0.31 & 5.99 & 17613 & protein \\
\hline 57 & Q5ZF92 & Eukaryotic translation initiation factor $5 \mathrm{~A}-1$ & P. major & 8 & 111 & 0.31 & 5.57 & 17486 & protein \\
\hline 58 & A8IZZ4 & Bi-ubiquitin & C. reinhardtii & 20 & 47 & 0.31 & 6.06 & 17185 & protein \\
\hline 59 & $\mathrm{~K} 3 \mathrm{XXC5}$ & Uncharacterized protein & S. italica & 43 & 319 & 0.30 & 7.06 & 42409 & glycolysis \\
\hline 60 & E4MWP5 & mRNA, clone: RTFL01-15-G22 & T. halophila & 17 & 116 & 0.30 & 6.16 & 35258 & photosynthesis \\
\hline 61 & D6PPS8 & 6-phosphogluconate dehydrogenase family protein & C. grandiflora & 4 & 144 & 0.30 & 9.22 & 21010 & OPP \\
\hline 62 & K3Z5U9 & Uncharacterized protein & S. italica & 34 & 400 & 0.30 & 6.47 & 49826 & photosynthesis \\
\hline 63 & K3XJN7 & Uncharacterized protein & S. italica & 37 & 468 & 0.30 & 7.85 & 35629 & TCA \\
\hline
\end{tabular}




\begin{tabular}{|c|c|c|c|c|c|c|c|c|c|}
\hline 64 & Q706C9 & GDP dissociation inhibitor & M. truncatula & 16 & 214 & 0.30 & 5.88 & 50211 & signalling \\
\hline 65 & D3G6B9 & Snakin-1 & S. bulbocastanum & 8 & 57 & 0.29 & 8.72 & 10442 & hormone metabolism \\
\hline 66 & B6TN41 & Regulator of ribonuclease activity A & Z. mays & 5 & 45 & 0.29 & 6.18 & 18390 & C1-metabolism \\
\hline 67 & E4MWV7 & mRNA, clone: RTFL01-09-M07 & T. halophila & 4 & 54 & 0.29 & 7.42 & 10792 & protein \\
\hline 68 & Q8LC80 & Putative calcium-binding protein, calreticulin & A. thaliana & 26 & 509 & 0.28 & 4.53 & 48385 & signalling \\
\hline 69 & F2D5B1 & Predicted protein & H. vulgare & 2 & 77 & 0.28 & 9 & 10876 & mitoETC \\
\hline 70 & K3Z913 & Uncharacterized protein & S. italica & 24 & 441 & 0.28 & 9.47 & 26793 & cell \\
\hline 71 & Q9SAT7 & Superoxide dismutase $[\mathrm{Cu}-\mathrm{Zn}]$ & P. tremuloides & 14 & 101 & 0.27 & 5.82 & 15420 & redox \\
\hline 72 & G5DXP1 & Fructose-bisphosphate aldolase & S. latifolia & 15 & 322 & 0.27 & 6.16 & 42771 & photosynthesis \\
\hline 73 & Q58H58 & Chloroplast photosynthetic oxygen-evolving protein $33 \mathrm{kDa}$ subunit & N. benthamiana & 20 & 160 & 0.27 & 5.66 & 35374 & photosynthesis \\
\hline 74 & G0WP59 & Superoxide dismutase $[\mathrm{Cu}-\mathrm{Zn}]$ & W. somnifera & 17 & 157 & 0.27 & 5.64 & 15782 & redox \\
\hline 75 & Q3HVN5 & Dehydroascorbate reductase & S. tuberosum & 5 & 137 & 0.26 & 6.54 & 23596 & redox \\
\hline 76 & K3YR06 & Uncharacterized protein & S. italica & 23 & 627 & 0.26 & 6.16 & 64225 & protein \\
\hline 77 & Q43874 & Ribulose bisphosphate carboxylase small chain & P. vulgaris & 17 & 167 & 0.26 & 9.03 & 20355 & photosynthesis \\
\hline 78 & Q06Z74 & Fibrillin 4 & C. canephora & 7 & 124 & 0.26 & 8.88 & 32064 & cell \\
\hline 79 & K3ZUR5 & Uncharacterized protein & S. italica & 11 & 368 & 0.26 & 6.24 & 36749 & co-factor and vitamine metabolism \\
\hline 80 & D2KZ81 & S-adenosylmethionine synthase & M. domestica & 8 & 368 & 0.25 & 4.94 & 24831 & amino acid metabolism \\
\hline 81 & Q069K2 & 5-methyltetrahydropteroyltriglutamate--homocysteine methyltransferase & N. suaveolens & 66 & 786 & 0.24 & 6.62 & 85060 & amino acid metabolism \\
\hline 82 & A9CM22 & Voltage-dependent anion channel & N. tabacum & 10 & 126 & 0.24 & 8.32 & 29694 & transport \\
\hline 83 & Q43796 & Inorganic pyrophosphatase & N. tabacum & 16 & 199 & 0.24 & 5.34 & 56198 & transport \\
\hline 84 & A1BLP6 & Thioredoxin & M. truncatula & 10 & 55 & 0.23 & 6.16 & 12904 & redox \\
\hline 85 & B6EBD6 & Heat shock protein $90-2$ & G. $\max$ & 29 & 423 & 0.23 & 5.07 & 80392 & stress \\
\hline 86 & D0EJY9 & Molecular chaperone Hsp90-3 & N. benthamiana & 24 & 420 & 0.23 & 5.02 & 80638 & stress \\
\hline 87 & Q9SRV5 & 5-methyltetrahydropteroyltriglutamate--homocysteine methyltransferase & A. thaliana & 96 & 1022 & 0.23 & 6.51 & 84873 & amino acid metabolism \\
\hline 88 & Q84XR5 & GCPE protein & C. roseus & 31 & 239 & 0.23 & 5.92 & 82522 & secondary metabolism \\
\hline 89 & F8RW92 & Peptidyl-prolyl cis-trans isomerase & H. brasiliensis & 8 & 171 & 0.22 & 8.44 & 18210 & cell \\
\hline 90 & E3NYI6 & Peptidyl-prolyl cis-trans isomerase & A. diogoi & 17 & 97 & 0.22 & 8.44 & 18332 & cell \\
\hline 91 & Q4H1G2 & Methionine synthase & B. vulgaris & 112 & 1323 & 0.22 & 6.46 & 88546 & amino acid metabolism \\
\hline 92 & A8MRE8 & Triosephosphate isomerase & A. thaliana & 10 & 110 & 0.22 & 7.49 & 32458 & photosynthesis \\
\hline 93 & D6PAY2 & Peptidyl-prolyl cis-trans isomerase & Vanda hybrid cultivar & 30 & 397 & 0.22 & 8.46 & 18582 & cell \\
\hline 94 & O81609 & Nodule-enhanced malate dehydrogenase & P. sativum & 19 & 514 & 0.22 & 7.75 & 42106 & TCA \\
\hline 95 & $\mathrm{D} 2 \mathrm{X} 5 \mathrm{~K} 2$ & DEAD-box RNA helicase-like protein & P. persica & 20 & 395 & 0.21 & 5.69 & 47191 & protein \\
\hline
\end{tabular}




\begin{tabular}{|c|c|c|c|c|c|c|c|c|c|}
\hline 96 & O48903 & Malate dehydrogenase & M. sativa & 18 & 226 & 0.21 & 8.29 & 38397 & gluconeogenesis \\
\hline 97 & F2CRM1 & Predicted protein & H. vulgare & 36 & 410 & 0.21 & 5.83 & 43138 & metal handling \\
\hline 98 & Q9FE12 & Peroxiredoxin & P. vulgaris & 15 & 250 & 0.21 & 5.33 & 28776 & redox \\
\hline 99 & B6TGL7 & Enolase & Z. mays & 10 & 368 & 0.20 & 6.23 & 50512 & glycolysis \\
\hline 100 & B4FPK8 & Cytochrome b5 & Z. mays & 6 & 156 & 0.20 & 5.3 & 14958 & redox \\
\hline 102 & A5Z1Y7 & Cytochrome b5 & M. domestica & 3 & 87 & 0.19 & 5.52 & 15243 & redox \\
\hline 101 & F2DAU4 & Predicted protein & H. vulgare & 15 & 231 & 0.19 & 7.56 & 62696 & protein \\
\hline 103 & H6VP90 & ATP synthase subunit B. & C. lobatus & 16 & 247 & 0.19 & 5.19 & 47813 & photosynthesis \\
\hline 104 & Q84P56 & TGB12K interacting protein 2 & N. tabacum & 14 & 80 & 0.19 & 4.55 & 37547 & RNA \\
\hline 105 & F2CQP8 & Predicted protein & H. vulgare & 14 & 153 & 0.18 & 5.49 & 32811 & biodegradation of xenobiotics \\
\hline 106 & K3ZXR4 & Uncharacterized protein & S. italica & 4 & 118 & 0.18 & 7.24 & 16069 & cell \\
\hline 108 & D7UPN0 & SEC13 family protein & L. japonicus & 8 & 53 & 0.18 & 6.01 & 32940 & protein \\
\hline 107 & E5GCQ8 & Inorganic pyrophosphatase & C. melo & 7 & 145 & 0.18 & 5.78 & 33109 & nucleotide metabolism \\
\hline 109 & B6TPE4 & Glutamate-1-semialdehyde 2,1-aminomutase & Z. mays & 6 & 81 & 0.18 & 6.55 & 50223 & tetrapyrrole synthesis \\
\hline 110 & K4A7I4 & Uncharacterized protein & S. italica & 20 & 358 & 0.18 & 5.4 & 61666 & photosynthesis \\
\hline 111 & A5YRY0 & Alpha-tubulin 3 & P. tremuloides & 6 & 118 & 0.18 & 5.01 & 50353 & cell \\
\hline 112 & O78327 & Transketolase 1 & C. аппиит & 36 & 212 & 0.17 & 6.62 & 80398 & OPP \\
\hline 113 & F2D1V6 & Predicted protein & H. vulgare & 6 & 83 & 0.17 & 6.6 & 34598 & minor $\mathrm{CHO}$ metabolism \\
\hline 114 & F2DBE0 & Predicted protein & H. vulgare & 6 & 144 & 0.17 & 8.22 & 34595 & TCA \\
\hline 115 & D8L9Q5 & Fructose-bisphosphate aldolase & T. aestivum & 12 & 299 & 0.17 & 8.09 & 41564 & photosynthesis \\
\hline 116 & B6UDL5 & Hydroxymethylbutenyl 4-diphosphate synthase & Z. mays & 26 & 205 & 0.17 & 5.97 & 82691 & secondary metabolism \\
\hline 117 & Q7XAE2 & Putative fructokinase 2 & P. integrifolia & 11 & 39 & 0.17 & 5.35 & 35181 & major $\mathrm{CHO}$ metabolism \\
\hline 118 & K3ZXP5 & Uncharacterized protein & S. italica & 9 & 50 & 0.17 & 6.8 & 17359 & protein \\
\hline 119 & K3XIT0 & Uncharacterized protein & S. italica & 19 & 375 & 0.17 & 8.78 & 41915 & photosynthesis \\
\hline 120 & Q5S7Y5 & Triosephosphate isomerase & C. reinhardtii & 18 & 87 & 0.16 & 7.65 & 30433 & photosynthesis \\
\hline 121 & E4MVY3 & mRNA, clone: RTFL01-01-M18 & T. halophila & 24 & 370 & 0.16 & 6.25 & 94734 & protein \\
\hline 122 & D7SS06 & Putative uncharacterized protein & V. vinifera & 10 & 254 & 0.16 & 5.38 & 68964 & transport \\
\hline 123 & E4MY36 & mRNA, clone: RTFL01-37-B16 & T. halophila & 16 & 131 & 0.15 & 6.25 & 63555 & glycolysis \\
\hline 124 & Q8LBY1 & Hydroxymethyltransferase & A. thaliana & 8 & 126 & 0.15 & 7.02 & 52129 & C1-metabolism \\
\hline 125 & B6TJT2 & Auxin-induced protein PCNT115 & Z. mays & 8 & 124 & 0.15 & 6.73 & 38553 & hormone metabolism \\
\hline 126 & K3Z7I7 & Uncharacterized protein & S. italica & 7 & 128 & 0.15 & 6.19 & 38341 & misc \\
\hline 127 & K3ZVK5 & Uncharacterized protein & S. italica & 19 & 33 & 0.15 & 6.57 & 32436 & photosynthesis \\
\hline
\end{tabular}




\begin{tabular}{|c|c|c|c|c|c|c|c|c|c|}
\hline 128 & D3GQL1 & Aconitate hydratase 3 & C. clementina & 25 & 131 & 0.15 & 6.3 & 98669 & TCA \\
\hline 129 & Q8LG34 & 6-phosphogluconate dehydrogenase, decarboxylating & A. thaliana & 6 & 226 & 0.15 & 5.8 & 53555 & OPP \\
\hline 130 & K3ZXB8 & Uncharacterized protein & S. italica & 4 & 44 & 0.14 & 8.6 & 19603 & protein \\
\hline 131 & $\mathrm{O} 23962$ & Translation elongation factor-TU & G. $\max$ & 5 & 37 & 0.14 & 5.19 & 27275 & protein \\
\hline 135 & B6T6Q8 & Alcohol dehydrogenase class 3 & Z. mays & 8 & 232 & 0.14 & 7.02 & 41639 & misc \\
\hline 132 & B6T9G3 & Alpha-1,4-glucan-protein synthase 1 & Z. mays & 7 & 166 & 0.14 & 7.05 & 41278 & cell wall \\
\hline 133 & K3Y823 & Uncharacterized protein & S. italica & 15 & 48 & 0.14 & 6.71 & 41387 & hormone metabolism \\
\hline 134 & Q6VWJ5 & Fructokinase 3 & S. lycopersicum & 6 & 121 & 0.14 & 5.8 & 41803 & major $\mathrm{CHO}$ metabolism \\
\hline 136 & A9ZMZ4 & Acetyl-CoA C-acetyltransferase & H. brasiliensis & 5 & 47 & 0.14 & 7.33 & 41798 & secondary metabolism \\
\hline 137 & B9ILW3 & Predicted protein & P. trichocarpa & 2 & 129 & 0.13 & 6.25 & 35485 & minor $\mathrm{CHO}$ metabolism \\
\hline 138 & B9SGJ6 & Ketol-acid reductoisomerase, chloroplast, putative & R. communis & 10 & 337 & 0.13 & 6.8 & 63712 & amino acid metabolism \\
\hline 139 & D7LW57 & Ketol-acid reductoisomerase & A. lyrata & 10 & 408 & 0.13 & 6.8 & 64346 & amino acid metabolism \\
\hline 140 & Q0W9E2 & B. chaperonin 60 & S. commersonii & 14 & 431 & 0.13 & 7.27 & 63333 & protein \\
\hline 141 & F2DWX8 & Predicted protein & H. vulgare & 3 & 65 & 0.13 & 6.39 & 21692 & lipid metabolism \\
\hline 142 & K3ZSX0 & Uncharacterized protein & S. italica & 5 & 193 & 0.13 & 7.64 & 53564 & amino acid metabolism \\
\hline 143 & F2DLJ7 & Predicted protein & H. vulgare & 12 & 163 & 0.13 & 7.77 & 44423 & hormone metabolism \\
\hline 145 & E4MVQ7 & mRNA, clone: RTFL01-03-C18 & T. halophila & 11 & 263 & 0.12 & 6.6 & 46631 & redox \\
\hline 144 & K4A6V8 & Uncharacterized protein & S. italica & 9 & 139 & 0.12 & 7.03 & 70549 & glycolysis \\
\hline 146 & K3XVH0 & Uncharacterized protein & S. italica & 22 & 141 & 0.12 & 6.62 & 80203 & photosynthesis \\
\hline 148 & B6SHL1 & Photosystem I reaction center subunit XI & Z. mays & 2 & 41 & 0.12 & 9.95 & 23193 & photosynthesis \\
\hline 147 & $\mathrm{~K} 3 \mathrm{XZA} 2$ & Uncharacterized protein & S. italica & 17 & 329 & 0.12 & 9.58 & 23221 & cell \\
\hline 149 & B6SVI5 & Putative uncharacterized protein & Z. mays & 10 & 34 & 0.12 & 8.67 & 24337 & not assigned \\
\hline 150 & E5LCX1 & C14 cysteine protease & S. demissum & 3 & 131 & 0.12 & 4.98 & 23974 & protein \\
\hline 151 & I0CC95 & Type II peroxiredoxin & T. hispida & 4 & 62 & 0.12 & 8.31 & 24226 & redox \\
\hline 152 & O49045 & Heat shock 70 protein & S. oleracea & 9 & 251 & 0.12 & 5.67 & 72558 & stress \\
\hline 153 & E4MXI2 & mRNA, clone: RTFL01-39-D20 & T. halophila & 14 & 343 & 0.12 & 5.21 & 73890 & stress \\
\hline 154 & B3TLP3 & Translation elongation factor EF-1 B. chain & E. guineensis & 5 & 90 & 0.11 & 4.69 & 24523 & protein \\
\hline 155 & K3YG24 & Uncharacterized protein & S. italica & 18 & 229 & 0.11 & 7.31 & 107135 & TCA \\
\hline 156 & A3QQY2 & Sucrose synthase & C. intybus & 21 & 242 & 0.11 & 6.39 & 92338 & major $\mathrm{CHO}$ metabolism \\
\hline 157 & B4G194 & $40 \mathrm{~S}$ ribosomal protein SA & Z. mays & 11 & 39 & 0.11 & 5.08 & 33640 & protein \\
\hline 158 & B6ZK00 & Peroxisomal biogenesis factor 11 family protein & G. $\max$ & 2 & 89 & 0.11 & 9.76 & 26114 & cell \\
\hline 160 & F2CXV7 & Predicted protein & H. vulgare & 7 & 322 & 0.11 & 8.05 & 25809 & protein \\
\hline
\end{tabular}




\begin{tabular}{|c|c|c|c|c|c|c|c|c|c|}
\hline 159 & F2DP49 & Predicted protein & H. vulgare & 11 & 181 & 0.11 & 8.63 & 25913 & hormone metabolism \\
\hline 162 & Q8VYN9 & Putative uncharacterized protein At5g54430 & A. thaliana & 2 & 62 & 0.11 & 6.57 & 26414 & stress \\
\hline 161 & Q93YG1 & Monodehydroascorbate reductase & M. crystalL. & 28 & 142 & 0.11 & 6.81 & 51944 & redox \\
\hline 164 & K3XL01 & Uncharacterized protein & S. italica & 4 & 54 & 0.10 & 9.09 & 27473 & photosynthesis \\
\hline 163 & K4ADT5 & Uncharacterized protein & S. italica & 2 & 154 & 0.10 & 5.97 & 26876 & $\operatorname{misc}$ \\
\hline 165 & O65156 & Glutathione peroxidase & Z. aethiopica & 5 & 121 & 0.10 & 9.73 & 26916 & redox \\
\hline 166 & Q5GAQ3 & Putative alanine aminotransferase & Z. mays & 8 & 150 & 0.10 & 5.64 & 57003 & amino acid metabolism \\
\hline 167 & $\mathrm{O} 22143$ & Transketolase-2, chloroplastic & A. thaliana & 8 & 136 & 0.10 & 5.96 & 69264 & OPP \\
\hline 168 & K3XL18 & Uncharacterized protein & S. italica & 6 & 90 & 0.10 & 6.28 & 28593 & mitoETC \\
\hline 169 & A9PAK9 & Putative uncharacterized protein & P. trichocarpa & 6 & 54 & 0.09 & 6.89 & 50623 & amino acid metabolism \\
\hline 170 & A5JVD4 & Putative uncharacterized protein & B. campestris & 8 & 54 & 0.09 & 6.99 & 30584 & cell wall \\
\hline 171 & F2CXX0 & Predicted protein & H. vulgare & 3 & 31 & 0.09 & 5.5 & 30839 & photosynthesis \\
\hline 172 & Q0MX14 & Putative aquaporin & V. vinifera & 3 & 66 & 0.09 & 8.15 & 30470 & transport \\
\hline 173 & B1NHU0 & Aquaporin PIP1-2 & G. hirsutum & 6 & 41 & 0.09 & 8.4 & 31129 & transport \\
\hline 174 & B4FH62 & NAD-dependent epimerase/dehydratase & Z. mays & 16 & 279 & 0.09 & 9.04 & 31917 & not assigned \\
\hline 177 & Q40270 & RNA-binding protein & M. crystalL. & 5 & 73 & 0.09 & 4.79 & 32001 & RNA \\
\hline 175 & Q40556 & Protein phosphatase $2 \mathrm{~A}$ & N. tabacum & 3 & 49 & 0.09 & 5.17 & 66079 & protein \\
\hline 176 & Q5PY86 & NADH:cytochrome b5 reductase & V. fordii & 5 & 35 & 0.09 & 7.15 & 31341 & redox \\
\hline 180 & A8JCE1 & Predicted protein & C. reinhardtii & 15 & 36 & 0.08 & 7.94 & 33842 & protein \\
\hline 181 & A9ZN09 & 2-C-methyl-D-erythritol 4-phosphate cytidylyltransferase & H. brasiliensis & 10 & 48 & 0.08 & 7.28 & 34509 & secondary metabolism \\
\hline 179 & B3TM50 & $26 \mathrm{~S}$ proteasome regulatory particle non-ATPase subunit 8 & E. guineensis & 5 & 29 & 0.08 & 6.28 & 34814 & protein \\
\hline 178 & F2E397 & Predicted protein & H. vulgare & 5 & 108 & 0.08 & 5.87 & 34933 & major $\mathrm{CHO}$ metabolism \\
\hline 184 & B3VMR3 & Epoxide hydrolase & N. benthamiana & 3 & 41 & 0.08 & 6.46 & 35842 & misc \\
\hline 183 & B6TB29 & Fructokinase-2 & Z. mays & 18 & 61 & 0.08 & 5.58 & 35875 & major $\mathrm{CHO}$ metabolism \\
\hline 182 & Q8LCW6 & Similar to late embryogenesis abundant proteins & A. thaliana & 6 & 57 & 0.08 & 4.87 & 36056 & development \\
\hline 185 & B9GYJ8 & Predicted protein & P. trichocarpa & 2 & 59 & 0.07 & 4.67 & 37573 & misc \\
\hline 190 & C5Z0K4 & Putative uncharacterized protein Sb09g004370 & S. bicolor & 6 & 49 & 0.07 & 6.87 & 40310 & redox \\
\hline 191 & E4MW60 & mRNA, clone: RTFL01-03-M14 & T. halophila & 6 & 50 & 0.07 & 7.42 & 39457 & secondary metabolism \\
\hline 186 & G5DWR0 & Branched-chain-amino-acid aminotransferase & S. latifolia & 5 & 97 & 0.07 & 5.83 & 38581 & amino acid metabolism \\
\hline 187 & K3XJA4 & Uncharacterized protein & S. italica & 2 & 101 & 0.07 & 8.07 & 39886 & C1-metabolism \\
\hline 189 & O24153 & S-adenosyl-methionine-sterol-C-methyltransferase homolog & N. tabacum & 2 & 46 & 0.07 & 7.55 & 39707 & hormone metabolism \\
\hline 188 & Q645M9 & Glyoxisomal malate dehydrogenase & S. lycopersicum & 3 & 121 & 0.07 & 7.94 & 38007 & gluconeogenesis \\
\hline
\end{tabular}




\begin{tabular}{|c|c|c|c|c|c|c|c|c|c|}
\hline 192 & Q9AXR6 & ATP:citrate lyase & C. аппиит & 11 & 54 & 0.07 & 7.39 & 66376 & TCA \\
\hline 193 & K3XWI6 & Uncharacterized protein & S. italica & 11 & 189 & 0.07 & 6.6 & 55489 & glycolysis \\
\hline 194 & E5GBJ6 & Alcohol dehydrogenase & C. melo & 3 & 76 & 0.07 & 8.47 & 40658 & misc \\
\hline 195 & Q9ZNX1 & NAD-dependent isocitrate dehydrogenase & N. tabacum & 3 & 114 & 0.07 & 7.52 & 40651 & TCA \\
\hline 197 & B4FYM0 & Acetyl-CoA acetyltransferase, cytosolic 2 & Z. mays & 3 & 137 & 0.06 & 7.66 & 43450 & secondary metabolism \\
\hline 196 & F2CTA9 & Predicted protein & H. vulgare & 4 & 75 & 0.06 & 4.75 & 42321 & cell \\
\hline 198 & F2CTR2 & Predicted protein & H. vulgare & 8 & 84 & 0.06 & 6.99 & 59167 & protein \\
\hline 199 & A5AW47 & Profilin & V. vinifera & 21 & 157 & 0.06 & 6.16 & 92852 & cell \\
\hline 204 & A9P9A2 & Putative uncharacterized protein & P. trichocarpa & 8 & 32 & 0.06 & 9.36 & 46272 & RNA \\
\hline 203 & E5GBW6 & $26 \mathrm{~S}$ proteasome non-ATPase regulatory subunit & C. melo & 2 & 38 & 0.06 & 5.92 & 44456 & protein \\
\hline 200 & F2DEC1 & Predicted protein & H. vulgare & 2 & 66 & 0.06 & 5.08 & 44443 & development \\
\hline 202 & Q1KUM6 & Putative uncharacterized protein & C. spinosa & 4 & 66 & 0.06 & 4.81 & 45374 & protein \\
\hline 201 & Q4ZJ73 & 12-oxophytodienoate reductase & H. brasiliensis & 4 & 66 & 0.06 & 8.72 & 44571 & hormone metabolism \\
\hline 206 & I1LCM5 & Uncharacterized protein & G. $\max$ & 2 & 51 & 0.06 & 6.96 & 47008 & TCA \\
\hline 205 & K3ZT72 & Uncharacterized protein & S. italica & 5 & 88 & 0.06 & 9.48 & 47497 & lipid metabolism \\
\hline 208 & A9RZ64 & Predicted protein & P. patens & 3 & 124 & 0.05 & 7.87 & 50746 & amino acid metabolism \\
\hline 207 & B9S7T6 & Transaminase mtnE, putative & R. communis & 6 & 55 & 0.05 & 7.28 & 50909 & amino acid metabolism \\
\hline 212 & B9UP05 & Hydroxymethylbutenyl diphosphate reductase & Oncidium hybrid cultivar & 3 & 56 & 0.05 & 6.2 & 52389 & secondary metabolism \\
\hline 209 & F2DDG9 & Predicted protein & H. vulgare & 6 & 100 & 0.05 & 5.66 & 52441 & cell \\
\hline 210 & P93698 & Lipoxygenase & V. unguiculata & 10 & 207 & 0.05 & 6.49 & 102731 & hormone metabolism \\
\hline 211 & Q8L935 & B.-1,3-glucanase-like protein & A. thaliana & 3 & 38 & 0.05 & 9.39 & 48952 & misc \\
\hline 214 & K4A938 & Uncharacterized protein & S. italica & 2 & 41 & 0.05 & 6.79 & 53547 & nucleotide metabolism \\
\hline 215 & $\mathrm{O} 24135$ & Citrate synthase & N. tabacum & 3 & 89 & 0.05 & 7.85 & 52744 & TCA \\
\hline 213 & Q9S768 & Alanine aminotransferase & O. sativa & 4 & 190 & 0.05 & 6.65 & 53130 & amino acid metabolism \\
\hline 217 & $\mathrm{~K} 3 \mathrm{XWC} 3$ & Uncharacterized protein & S. italica & 3 & 171 & 0.05 & 9 & 58110 & development \\
\hline 216 & K3YH65 & Uncharacterized protein & S. italica & 2 & 58 & 0.05 & 7.56 & 56313 & amino acid metabolism \\
\hline 220 & A8JIB7 & Chaperonin 60A & C. reinhardtii & 3 & 87 & 0.04 & 5.62 & 61911 & photosynthesis \\
\hline 222 & B9ICC7 & Predicted protein & P. trichocarpa & 4 & 52 & 0.04 & 4.78 & 60802 & signalling \\
\hline 218 & H9A1W2 & Acyl-activating enzyme 10 & C. sativa & 3 & 49 & 0.04 & 6.49 & 62013 & lipid metabolism \\
\hline 221 & Q84RQ1 & Calcium-dependent protein kinase & S. oligorhiza & 2 & 125 & 0.04 & 7.23 & 61263 & signalling \\
\hline 219 & Q9SA89 & FAD-binding and BBE domain-containing protein & A. thaliana & 5 & 76 & 0.04 & 8.6 & 60060 & misc \\
\hline 223 & B2KNE6 & Phospholipase D & H. annuиs & 9 & 144 & 0.04 & 5.52 & 92315 & lipid metabolism \\
\hline
\end{tabular}




\begin{tabular}{|c|c|c|c|c|c|c|c|c|c|}
\hline 224 & K3ZC75 & Uncharacterized protein & S. italica & 9 & 37 & 0.04 & 4.72 & 96887 & protein \\
\hline 225 & F8WQA8 & S9 Tyrosyl aminopeptidase & R. sativus & 5 & 97 & 0.03 & 5.45 & 76789 & protein \\
\hline 226 & E4MW95 & mRNA, clone: RTFL01-05-M24 & T. halophila & 3 & 74 & 0.03 & 5.45 & 79691 & misc \\
\hline 227 & Q9XFX7 & Phospholipase D & C. plantagineum & 8 & 34 & 0.03 & 5.66 & 92228 & lipid metabolism \\
\hline 228 & Q9ZVY6 & $\mathrm{T} 25 \mathrm{~N} 20.17$ & A. thaliana & 3 & 56 & 0.03 & 5.58 & 90732 & protein \\
\hline 229 & Q6E438 & ACT11D09.4 & C. melo & 5 & 106 & 0.03 & 7.93 & 100881 & not assigned \\
\hline 230 & K3ZH11 & Uncharacterized protein & S. italica & 11 & 67 & 0.02 & 5.38 & 118046 & protein \\
\hline
\end{tabular}

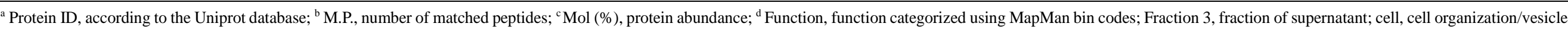

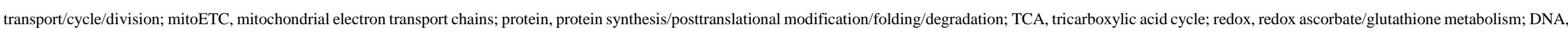
DNA synthesis/repair; OPP, oxidative pentose phosphate; RNA, RNA processing/regulation of transcription; and misc, miscellaneous. 
Supplemental Table 10. Crude Extract Proteins Identified in L. japonica Immature Flower Buds.

\begin{tabular}{|c|c|c|c|c|c|c|c|c|c|}
\hline No. & Protein $\mathrm{ID}^{\mathrm{a}}$ & Description & Species & M.P. ${ }^{\mathrm{b}}$ & Score & $\operatorname{Mol}(\%)^{\mathrm{c}}$ & pI & Mass (Da) & Function $^{\mathrm{d}}$ \\
\hline 1 & H1ZY49 & Translation elongation factor 1-alpha & L. maackii & 51 & 413 & 1.58 & 5.00 & 11619 & protein \\
\hline 2 & B5M4B1 & Beta-tubulin & S. tuberosum & 111 & 1790 & 1.32 & 4.86 & 51166 & cell \\
\hline 3 & O82722 & ATP synthase subunit beta & N. sylvestris & 184 & 4136 & 1.22 & 6.06 & 59638 & mitoETC \\
\hline 4 & C4B8E5 & Glyceraldehyde-3-phosphate dehydrogenase & T. gesneriana & 64 & 1778 & 1.21 & 7.96 & 20964 & glycolysis \\
\hline 5 & K3Z679 & Uncharacterized protein & S. italica & 99 & 1356 & 1.13 & 4.88 & 50728 & cell \\
\hline 6 & C6GFP3 & ATP synthase subunit beta & G. hirsutum & 162 & 3341 & 0.94 & 6.29 & 59987 & mitoETC \\
\hline 7 & D6C638 & Ribulose-1,5-bisphosphate carboxylase/oxygenase large subunit & Psychotria sp. & 122 & 994 & 0.87 & 6.58 & 50770 & photosynthesis \\
\hline 8 & H6T8A4 & ATP synthase subunit beta & M. poeppigii & 68 & 1264 & 0.81 & 6.33 & 52110 & photosynthesis \\
\hline 9 & F2D884 & Predicted protein & H. vulgare & 169 & 3234 & 0.80 & 5.19 & 71476 & stress \\
\hline 10 & K3Z4G6 & Uncharacterized protein & S. italica & 174 & 2573 & 0.77 & 5.25 & 71367 & stress \\
\hline 11 & Q6PKU2 & Putative glyceraldehyde-3-phosphate dehydrogenase & O. minor & 53 & 1226 & 0.74 & 7.09 & 22122 & glycolysis \\
\hline 12 & A8MQP1 & Adenosylhomocysteinase 1 & A. thaliana & 72 & 855 & 0.64 & 5.16 & 36022 & amino acid metabolism \\
\hline 13 & E5GBV9 & Glyceraldehyde-3-phosphate dehydrogenase & C. melo & 71 & 768 & 0.64 & 6.62 & 36471 & glycolysis \\
\hline 14 & A7KQH0 & Beta-tubulin & E. grandis & 76 & 885 & 0.62 & 4.94 & 50615 & cell \\
\hline 15 & J3RTS9 & Glyceraldehyde-3-phosphate dehydrogenase & C. pentagona & 88 & 1832 & 0.58 & 6.72 & 32382 & glycolysis \\
\hline 16 & A4ZW20 & Granule-bound starch synthase I & V. foetidum & 28 & 182 & 0.56 & 6.60 & 22721 & protein \\
\hline 17 & Q9M6B3 & Malate dehydrogenase & V. vinifera & 71 & 2087 & 0.54 & 8.62 & 37137 & TCA \\
\hline 18 & Q08II7 & Heat shock protein 70-like protein & L. sativa & 15 & 571 & 0.54 & 10.33 & 12539 & stress \\
\hline 19 & B6SPX4 & Tubulin alpha- 3 chain & Z. mays & 53 & 669 & 0.50 & 5.02 & 50370 & cell \\
\hline 20 & E4MW44 & mRNA, clone: RTFL01-05-I15 & T. halophila & 92 & 607 & 0.49 & 5.58 & 42078 & cell \\
\hline 21 & $\mathrm{~F} 2 \mathrm{E} 4 \mathrm{C} 2$ & Predicted protein & H. vulgare & 132 & 1083 & 0.49 & 5.25 & 72202 & stress \\
\hline 22 & D9ZBV9 & Malate dehydrogenase & S. globosa & 7 & 156 & 0.48 & 4.92 & 13766 & TCA \\
\hline 23 & K3ZWC0 & Uncharacterized protein & S. italica & 15 & 334 & 0.47 & 10.64 & 28758 & protein \\
\hline 24 & K4ACE3 & Uncharacterized protein & S. italica & 73 & 1217 & 0.46 & 6.09 & 35803 & TCA \\
\hline 25 & Q4H1G1 & Adenosylhomocysteinase & B. vulgaris & 87 & 885 & 0.45 & 6.19 & 54052 & amino acid metabolism \\
\hline 26 & B6EBD6 & Heat shock protein $90-2$ & G. $\max$ & 120 & 1509 & 0.44 & 5.07 & 80392 & stress \\
\hline 27 & K4A9V6 & Uncharacterized protein & S. italica & 38 & 498 & 0.44 & 5.10 & 50519 & cell \\
\hline 28 & F2EG92 & Predicted protein & H. vulgare & 4 & 117 & 0.41 & 12.12 & 7602 & protein \\
\hline 29 & Q84RD8 & Adenosylhomocysteinase & M. truncatula & 67 & 923 & 0.40 & 6.01 & 53790 & amino acid metabolism \\
\hline 30 & J9QAL9 & Ribosomal protein L2 & A. corniculatum & 12 & 120 & 0.40 & 10.23 & 21703 & protein \\
\hline 31 & Q9FS70 & Putative granule bound starch synthase & T. grandis & 4 & 39 & 0.39 & 5.17 & 7851 & major $\mathrm{CHO}$ metabolism \\
\hline
\end{tabular}




\begin{tabular}{|c|c|c|}
\hline 32 & Q8MCY2 & Ribulose bisphosphate carboxylase large chain \\
\hline 33 & K3YQW5 & Uncharacterized protein \\
\hline 34 & D0EJY9 & Molecular chaperone Hsp90-3 \\
\hline 35 & E4MXI2 & mRNA, clone: RTFL01-39-D20 \\
\hline 36 & K3XJN7 & Uncharacterized protein \\
\hline 37 & Q645N1 & Malate dehydrogenase \\
\hline 38 & Q6T379 & Triosephosphate isomerase \\
\hline 40 & K3ZUR5 & Uncharacterized protein \\
\hline 39 & B3TLL4 & Triosephosphate isomerase \\
\hline 41 & D7NHW9 & 2-phospho-D-glycerate hydrolase \\
\hline 42 & B6SJZ8 & Glyceraldehyde-3-phosphate dehydrogenase, cytosolic \\
\hline 43 & Q43833 & Glyceraldehyde 3-phosphate dehydrogenase \\
\hline 44 & F2EFD1 & $40 \mathrm{~S}$ ribosomal protein $\mathrm{S} 27$ \\
\hline 45 & G5DVX2 & Phosphoglycerate kinase \\
\hline 46 & F2DAF1 & Predicted protein \\
\hline 47 & Q9AXR6 & ATP:citrate lyase \\
\hline 48 & K3XEI1 & Uncharacterized protein \\
\hline 49 & K3YR06 & Uncharacterized protein \\
\hline 50 & Q6ZXH7 & Putative ascorbate peroxidase \\
\hline 51 & K3Z5U9 & Uncharacterized protein \\
\hline 52 & $\mathrm{O} 22331$ & Glutamine synthetase \\
\hline 53 & H9NIE1 & Cell division cycle protein 48 \\
\hline 54 & B4F8L7 & Glyceraldehyde-3-phosphate dehydrogenase B \\
\hline 55 & B6TPE4 & Glutamate-1-semialdehyde 2,1-aminomutase \\
\hline 56 & F2DAU4 & Predicted protein \\
\hline 57 & B2VQE0 & Methionine synthase \\
\hline 58 & Q645M9 & Glyoxisomal malate dehydrogenase \\
\hline 59 & K3XFR6 & Uncharacterized protein \\
\hline 60 & K3XXC5 & Uncharacterized protein \\
\hline 61 & O81609 & Nodule-enhanced malate dehydrogenase \\
\hline 62 & E4MXI1 & mRNA, clone: RTFL01-39-B07 \\
\hline 63 & $\mathrm{O} 48903$ & Malate dehydrogenase \\
\hline 64 & F2D690 & Predicted protein \\
\hline
\end{tabular}

\begin{tabular}{|c|c|c|c|c|c|c|}
\hline V. baccifera & 63 & 525 & 0.38 & 6.64 & 48739 & photosynthesis \\
\hline S. italica & 38 & 878 & 0.37 & 5.39 & 68637 & transport \\
\hline N. benthamiana & 117 & 1669 & 0.37 & 5.02 & 80638 & stress \\
\hline T. halophila & 60 & 865 & 0.36 & 5.21 & 73890 & stress \\
\hline S. italica & 32 & 492 & 0.36 & 7.85 & 35629 & TCA \\
\hline S. lycopersicum & 27 & 982 & 0.35 & 8.72 & 36357 & TCA \\
\hline S. chacoense & 17 & 220 & 0.34 & 5.99 & 27251 & glycolysis \\
\hline S. italica & 18 & 536 & 0.34 & 6.24 & 36749 & co-factor and vitamine metabolism \\
\hline E. guineensis & 20 & 172 & 0.34 & 6.23 & 27476 & glycolysis \\
\hline P. trifoliata & 35 & 930 & 0.34 & 5.78 & 47986 & glycolysis \\
\hline Z. mays & 64 & 841 & 0.31 & 6.96 & 36614 & glycolysis \\
\hline S. tuberosum & 30 & 804 & 0.31 & 6.44 & 32598 & glycolysis \\
\hline H. vulgare & 2 & 48 & 0.30 & 8.72 & 9905 & protein \\
\hline S. latifolia & 76 & 1194 & 0.30 & 6.58 & 51352 & photosynthesis \\
\hline H. vulgare & 25 & 503 & 0.29 & 9.80 & 17888 & protein \\
\hline C. аппиит & 34 & 539 & 0.27 & 7.39 & 66376 & TCA \\
\hline S. italica & 60 & 792 & 0.26 & 6.27 & 94876 & protein \\
\hline S. italica & 52 & 1399 & 0.26 & 6.16 & 64225 & protein \\
\hline P. canadensis & 11 & 143 & 0.26 & 4.97 & 22506 & redox \\
\hline S. italica & 44 & 477 & 0.26 & 6.47 & 49826 & photosynthesis \\
\hline H. brasiliensis & 15 & 192 & 0.26 & 6.20 & 39504 & $\mathrm{~N}$-metabolism \\
\hline C. sinensis & 44 & 939 & 0.26 & 5.29 & 90644 & cell \\
\hline Z. mays & 17 & 269 & 0.26 & 6.39 & 47663 & photosynthesis \\
\hline Z. mays & 13 & 240 & 0.25 & 6.55 & 50223 & tetrapyrrole synthesis \\
\hline H. vulgare & 55 & 659 & 0.25 & 7.56 & 62696 & protein \\
\hline O. ramosa & 131 & 1248 & 0.25 & 6.40 & 84862 & amino acid metabolism \\
\hline S. lycopersicum & 16 & 379 & 0.24 & 7.94 & 38007 & gluconeogenesis \\
\hline S. italica & 27 & 505 & 0.23 & 7.46 & 66448 & TCA \\
\hline S. italica & 55 & 497 & 0.23 & 7.06 & 42409 & glycolysis \\
\hline P. sativum & 14 & 408 & 0.23 & 7.75 & 42106 & TCA \\
\hline T. halophila & 19 & 357 & 0.23 & 6.98 & 48063 & photosynthesis \\
\hline M. sativa & 19 & 351 & 0.23 & 8.29 & 38397 & gluconeogenesis \\
\hline H. vulgare & 5 & 163 & 0.23 & 4.37 & 12678 & protein \\
\hline
\end{tabular}




\begin{tabular}{|c|c|c|}
\hline 66 & Q9ATF4 & Ribosomal protein L33 \\
\hline 65 & C1M2W0 & Somatic embryogenesis cinnamyl alcohol dehydrogenase 1 \\
\hline 67 & O82089 & AT3g56240/F18O21_200 \\
\hline 68 & B4FL17 & Translationally-controlled tumor protein \\
\hline 69 & Q34691 & ATP synthase subunit alpha \\
\hline 70 & D0ELH5 & Peptidyl-prolyl cis-trans isomerase \\
\hline 71 & Q7XJH9 & Transaldolase \\
\hline 72 & Q8GZR6 & Hydroxymethylbutenyl 4-diphosphate synthase \\
\hline 73 & D3GQL1 & Aconitate hydratase 3 \\
\hline 74 & K3ZW60 & Uncharacterized protein \\
\hline 75 & K3XVH0 & Uncharacterized protein \\
\hline 76 & A8MQR4 & $60 \mathrm{~S}$ acidic ribosomal protein $\mathrm{P} 0-2$ \\
\hline 77 & H2BBB1 & ATP synthase subunit alpha, chloroplastic \\
\hline 78 & A9ZN14 & 4-hydroxy-3-methylbut-2-en-1-yl diphosphate synthase \\
\hline 79 & K4A7I4 & Uncharacterized protein \\
\hline 80 & Q9SAT7 & Superoxide dismutase $[\mathrm{Cu}-\mathrm{Zn}]$ \\
\hline 81 & E5GBS0 & $40 \mathrm{~S}$ ribosomal protein $\mathrm{S} 24$ \\
\hline 82 & Q0W9E2 & Beta chaperonin 60 \\
\hline 83 & B6UFB3 & Stromal $70 \mathrm{kDa}$ heat shock-related protein \\
\hline 84 & Q8LC80 & Putative calcium-binding protein, calreticulin \\
\hline 85 & K3YZ28 & Uncharacterized protein \\
\hline 86 & G5DXP1 & Fructose-bisphosphate aldolase \\
\hline 87 & B6TGT0 & $60 \mathrm{~S}$ ribosomal protein $\mathrm{L} 2$ \\
\hline 88 & Q42425 & Aspartate aminotransferase \\
\hline 89 & Q8LBY1 & Hydroxymethyltransferase \\
\hline 90 & Q1KUX5 & 6-phosphogluconate dehydrogenase, decarboxylating \\
\hline 91 & E1Z5J4 & Cytoplasmic ribosomal protein S13 \\
\hline 92 & Q6H8J2 & $40 \mathrm{~S}$ ribosomal protein S9 \\
\hline 94 & E1Z823 & $40 \mathrm{~S}$ ribosomal protein $\mathrm{S} 18$ \\
\hline 93 & B9RZK1 & Major allergen Pru ar, putative \\
\hline 95 & K4AAQ4 & Uncharacterized protein \\
\hline 96 & D6PAY2 & Peptidyl-prolyl cis-trans isomerase \\
\hline 97 & A4UMS1 & Myo-inositol-1-phosphate synthase isoform 1 \\
\hline
\end{tabular}

\begin{tabular}{|c|c|c|c|c|c|c|}
\hline C. sativa & 4 & 182 & 0.22 & 10.58 & 12831 & protein \\
\hline C. sativus & 27 & 286 & 0.22 & 7.44 & 39462 & secondary metabolism \\
\hline A. thaliana & 6 & 121 & 0.22 & 4.93 & 13077 & metal handling \\
\hline Z. mays & 8 & 80 & 0.21 & 4.67 & 18773 & development \\
\hline H. annuиs & 34 & 280 & 0.21 & 6.60 & 55766 & mitoETC \\
\hline C. sinensis & 5 & 169 & 0.21 & 8.46 & 18310 & cell \\
\hline S. lycopersicum & 18 & 331 & 0.20 & 5.96 & 48062 & OPP \\
\hline S. lycopersicum & 50 & 1173 & 0.20 & 6.11 & 82805 & secondary metabolism \\
\hline C. clementina & 41 & 462 & 0.19 & 6.30 & 98669 & TCA \\
\hline S. italica & 46 & 861 & 0.19 & 9.76 & 29913 & protein \\
\hline S. italica & 58 & 273 & 0.19 & 6.62 & 80203 & photosynthesis \\
\hline A. thaliana & 18 & 134 & 0.19 & 4.84 & 30656 & protein \\
\hline P. diguetii & 30 & 386 & 0.19 & 5.74 & 55710 & photosynthesis \\
\hline H. brasiliensis & 51 & 1067 & 0.18 & 6.47 & 82653 & secondary metabolism \\
\hline S. italica & 28 & 660 & 0.18 & 5.40 & 61666 & photosynthesis \\
\hline P. tremuloides & 7 & 94 & 0.18 & 5.82 & 15420 & redox \\
\hline C. melo & 11 & 240 & 0.18 & 10.64 & 15761 & protein \\
\hline S. commersonii & 39 & 1009 & 0.18 & 7.27 & 63333 & protein \\
\hline Z. mays & 35 & 270 & 0.18 & 5.21 & 74853 & stress \\
\hline A. thaliana & 15 & 400 & 0.18 & 4.53 & 48385 & signalling \\
\hline S. italica & 4 & 103 & 0.17 & 10.59 & 15971 & protein \\
\hline S. latifolia & 10 & 293 & 0.17 & 6.16 & 42771 & photosynthesis \\
\hline Z. mays & 6 & 91 & 0.17 & 11.08 & 28373 & protein \\
\hline P. miliaceum & 14 & 124 & 0.17 & 8.48 & 50477 & amino acid metabolism \\
\hline A. thaliana & 13 & 304 & 0.16 & 7.02 & 52129 & C1-metabolism \\
\hline C. spinosa & 14 & 202 & 0.16 & 6.06 & 53777 & OPP \\
\hline C. variabilis & 2 & 37 & 0.16 & 10.48 & 17007 & protein \\
\hline C. roseus & 4 & 41 & 0.16 & 10.20 & 23015 & protein \\
\hline C. variabilis & 13 & 40 & 0.16 & 10.27 & 17472 & protein \\
\hline R. communis & 2 & 61 & 0.16 & 4.98 & 17294 & stress \\
\hline S. italica & 16 & 650 & 0.15 & 10.56 & 44399 & protein \\
\hline V. hybrid cultivar & 15 & 292 & 0.15 & 8.46 & 18582 & cell \\
\hline X. viscosa & 16 & 255 & 0.15 & 5.41 & 56899 & minor $\mathrm{CHO}$ metabolism \\
\hline
\end{tabular}




\begin{tabular}{lll}
98 & O23963 & Elongation factor Tu \\
99 & A8MRE8 & Triosephosphate isomerase \\
100 & K3Z913 & Uncharacterized protein \\
101 & E4MX73 & mRNA, clone: RTFL01-10-N01 \\
102 & B3F8H6 & Sucrose synthase \\
103 & F6H3T7 & Putative uncharacterized protein \\
104 & Q19TV8 & UDP-glucose pyrophosphorylase \\
105 & B6T6Q8 & Alcohol dehydrogenase class 3 \\
106 & B3TLQ5 & 60S ribosomal protein L11 \\
107 & K3ZX77 & Uncharacterized protein \\
108 & B3TM23 & 40S ribosomal protein S4 \\
110 & Q4H1G2 & Methionine synthase \\
109 & D2IR31 & Granule-bound starch synthase \\
111 & O78327 & Uncharacterized protein \\
112 & Q9XGU8 & Isocitrate dehydrogenase [NADP] \\
113 & O22402 & GDP dissociation inhibitor \\
114 & K3YR95 & Uncharacterized protein \\
115 & A9CM22 & Voltage-dependent anion channel \\
116 & Q9STA4 & Cysteine protease \\
117 & F2E797 & Predicted protein \\
118 & A8MQA1 & 60S ribosomal protein L13 \\
119 & E5LCX1 & C14 cysteine protease \\
120 & B3TLP3 & Translation elongation factor EF-1 beta chain \\
121 & K3XLU5 & Uncharacterized protein \\
122 & K3Y9Y1 & Uncharacterized protein \\
123 & D2KZ81 & S-adenosylmethionine synthase \\
124 & E4MXR5 & mRNA, clone: RTFL01-40-M04 \\
127 & B6ZK00 & Peroxisomal biogenesis factor 11 family protein \\
126 & F2DP49 & Predicted protein \\
128 & F2CQY1 & Predicted protein \\
125 & F2DLC2 & Proteasome subunit alpha type \\
Q3V5S6 & K3XZ1a-conglycinin alpha subunit & Uncharacterized protein \\
\hline 10 & &
\end{tabular}

\begin{tabular}{|c|c|c|c|c|c|c|}
\hline G. $\max$ & 17 & 125 & 0.14 & 5.14 & 38044 & protein \\
\hline A. thaliana & 6 & 105 & 0.14 & 7.49 & 32458 & photosynthesis \\
\hline S. italica & 5 & 245 & 0.14 & 9.47 & 26793 & cell \\
\hline T. halophila & 12 & 386 & 0.14 & 5.83 & 39684 & TCA \\
\hline N. langsdorffii & 47 & 480 & 0.14 & 6.40 & 92814 & major CHO metabolism \\
\hline$V$. vinifera & 18 & 80 & 0.14 & 6.11 & 40651 & not assigned \\
\hline C. melo & 12 & 72 & 0.14 & 7.17 & 52201 & glycolysis \\
\hline Z. mays & 13 & 250 & 0.13 & 7.02 & 41639 & misc \\
\hline E. guineensis & 6 & 119 & 0.13 & 9.92 & 20950 & protein \\
\hline S. italica & 4 & 153 & 0.13 & 11.34 & 21045 & protein \\
\hline E. guineensis & 15 & 139 & 0.13 & 10.24 & 30023 & protein \\
\hline B. vulgaris & 75 & 1023 & 0.13 & 6.46 & 88546 & amino acid metabolism \\
\hline Z. mays & 20 & 247 & 0.13 & 6.84 & 66351 & major $\mathrm{CHO}$ metabolism \\
\hline C. аппиит & 36 & 254 & 0.13 & 6.62 & 80398 & photosynthesis \\
\hline O. sativa & 17 & 152 & 0.13 & 6.80 & 46356 & $\mathrm{TCA}$ \\
\hline N. tabacum & 14 & 84 & 0.13 & 5.67 & 50127 & signalling \\
\hline S. italica & 19 & 216 & 0.12 & 7.65 & 58776 & protein \\
\hline N. tabacum & 6 & 152 & 0.12 & 8.32 & 29694 & transport \\
\hline M. sativa & 2 & 44 & 0.12 & 5.57 & 22875 & protein \\
\hline H. vulgare & 19 & 161 & 0.12 & 5.72 & 47111 & $\mathrm{TCA}$ \\
\hline A. thaliana & 3 & 102 & 0.11 & 11.00 & 23525 & protein \\
\hline S. demissum & 6 & 236 & 0.11 & 4.98 & 23974 & protein \\
\hline E. guineensis & 6 & 171 & 0.11 & 4.69 & 24523 & protein \\
\hline S. italica & 3 & 32 & 0.11 & 10.08 & 24413 & protein \\
\hline S. italica & 7 & 53 & 0.11 & 10.07 & 24396 & protein \\
\hline M. domestica & 7 & 362 & 0.11 & 4.94 & 24831 & amino acid metabolism \\
\hline T. halophila & 9 & 180 & 0.11 & 5.90 & 73489 & stress \\
\hline G. $\max$ & 2 & 94 & 0.11 & 9.76 & 26114 & cell \\
\hline H. vulgare & 2 & 84 & 0.11 & 8.63 & 25913 & not assigned \\
\hline H. vulgare & 2 & 66 & 0.11 & 9.52 & 25529 & protein \\
\hline H. vulgare & 2 & 154 & 0.11 & 4.68 & 26087 & protein \\
\hline G. $\max$ & 8 & 239 & 0.10 & 5.15 & 70526 & RNA \\
\hline S. italica & 4 & 64 & 0.10 & 6.20 & 26286 & protein \\
\hline
\end{tabular}




\begin{tabular}{|c|c|c|}
\hline 131 & Q6L5N6 & Heat shock protein 70 \\
\hline 132 & K4A6V8 & Uncharacterized protein \\
\hline 134 & Q7XAE2 & Putative fructokinase 2 \\
\hline 133 & E4MWP5 & mRNA, clone: RTFL01-15-G22 \\
\hline 135 & A0FKE6 & Chloroplast threonine deaminase 1 \\
\hline 136 & K3XL18 & Uncharacterized protein \\
\hline 137 & B5LAV3 & Isopropylmalate synthase \\
\hline 138 & B6TNX7 & Proteasome subunit alpha type \\
\hline 139 & O04946 & Enoyl-ACP reductase \\
\hline 140 & Q948V5 & Mitochondrial processing peptidase alpha subunit \\
\hline 141 & F6HGZ1 & Pectinesterase \\
\hline 142 & A9P9A2 & Putative uncharacterized protein \\
\hline 143 & D7UPN0 & SEC13 family protein \\
\hline 144 & A9ZMZ5 & Acetyl-CoA C-acetyltransferase \\
\hline 145 & С0Р9J6 & Aminoaldehyde dehydrogenase 1 \\
\hline 146 & Q8LFI3 & Nuclear antigen homolog \\
\hline 147 & B9RI89 & Serine-threonine protein kinase, plant-type, putative \\
\hline 150 & Q39986 & Cysteine proteinase \\
\hline 148 & B8AYG0 & Putative uncharacterized protein \\
\hline 149 & C1IC54 & Cinnamyl alcohol dehydrogenase \\
\hline 151 & B9HJ23 & Predicted protein \\
\hline 153 & E5F725 & Putative uncharacterized protein \\
\hline 154 & Q8S917 & Phosphoenolpyruvate carboxylase \\
\hline 152 & B4FYM0 & Acetyl-CoA acetyltransferase, cytosolic 2 \\
\hline 155 & Q4LER6 & Beta-conglycinin alpha prime subunit \\
\hline 156 & K3XWI6 & Uncharacterized protein \\
\hline 157 & F2DLJ7 & Predicted protein \\
\hline 158 & E4MVQ7 & mRNA, clone: RTFL01-03-C18 \\
\hline 160 & A9PAK9 & Putative uncharacterized protein \\
\hline 159 & F2DDG9 & Predicted protein \\
\hline 162 & Q9M318 & Polygalacturonase-like protein \\
\hline 161 & Q8VWP8 & Acyltransferase-like protein \\
\hline 163 & Q93YG1 & Monodehydroascorbate reductase \\
\hline
\end{tabular}

\begin{tabular}{|c|c|c|c|c|c|c|}
\hline N. benthamiana & 2 & 60 & 0.10 & 5.26 & 26923 & stress \\
\hline S. italica & 9 & 95 & 0.10 & 7.03 & 70549 & glycolysis \\
\hline P. integrifolia & 8 & 49 & 0.10 & 5.35 & 35181 & major $\mathrm{CHO}$ metabolism \\
\hline T. halophila & 11 & 43 & 0.10 & 6.16 & 35258 & photosynthesis \\
\hline S. lycopersicum & 10 & 79 & 0.10 & 6.37 & 66653 & amino acid metabolism \\
\hline S. italica & 3 & 67 & 0.09 & 6.28 & 28593 & mitoETC \\
\hline C. аппиит & 6 & 59 & 0.09 & 6.54 & 66084 & amino acid metabolism \\
\hline Z. mays & 4 & 82 & 0.09 & 5.40 & 30582 & protein \\
\hline N. tabacum & 7 & 42 & 0.09 & 8.78 & 41925 & lipid metabolism \\
\hline M. alba & 6 & 138 & 0.08 & 8.25 & 54978 & protein \\
\hline$V$. vinifera & 9 & 186 & 0.08 & 7.49 & 63329 & cell wall \\
\hline P. trichocarpa & 8 & 170 & 0.08 & 9.36 & 46272 & RNA \\
\hline L. japonicus & 2 & 41 & 0.08 & 6.01 & 32940 & protein \\
\hline H. brasiliensis & 13 & 354 & 0.08 & 6.68 & 42039 & secondary metabolism \\
\hline Zea mays & 5 & 79 & 0.08 & 5.45 & 55733 & secondary metabolism \\
\hline A. thaliana & 2 & 34 & 0.07 & 8.73 & 37414 & RNA \\
\hline R. communis & 5 & 63 & 0.07 & 8.18 & 40375 & not assigned \\
\hline Hemerocallis sp. & 2 & 79 & 0.07 & 5.50 & 40006 & protein \\
\hline O. sativa & 8 & 39 & 0.07 & 6.99 & 40287 & redox \\
\hline G. hirsutum & 7 & 52 & 0.07 & 6.25 & 39574 & secondary metabolism \\
\hline P. trichocarpa & 13 & 258 & 0.06 & 6.54 & 101700 & misc \\
\hline E. parvulum & 2 & 153 & 0.06 & 7.11 & 42041 & cell wall \\
\hline N. sylvestris & 7 & 116 & 0.06 & 6.74 & 86261 & glycolysis \\
\hline Z. mays & 7 & 95 & 0.06 & 7.66 & 43450 & secondary metabolism \\
\hline G. $\max$ & 2 & 58 & 0.06 & 5.71 & 72469 & development \\
\hline S. italica & 14 & 196 & 0.06 & 6.60 & 55489 & glycolysis \\
\hline H. vulgare & 4 & 133 & 0.06 & 7.77 & 44423 & not assigned \\
\hline T. halophila & 8 & 156 & 0.06 & 6.60 & 46631 & redox \\
\hline P. trichocarpa & 6 & 71 & 0.05 & 6.89 & 50623 & amino acid metabolism \\
\hline H. vulgare & 2 & 46 & 0.05 & 5.66 & 52441 & cell \\
\hline A. thaliana & 2 & 141 & 0.05 & 6.29 & 52533 & cell wall \\
\hline G. hirsutum & 2 & 146 & 0.05 & 5.96 & 48286 & not assigned \\
\hline M. crystallinum & 15 & 144 & 0.05 & 6.81 & 51944 & redox \\
\hline
\end{tabular}




\begin{tabular}{|c|c|c|c|c|c|c|c|c|}
\hline Q9S768 & Alanine aminotransferase & O. sativa & 3 & 147 & 0.05 & 6.65 & 53130 & amino acid metabolism \\
\hline K3YRV8 & Uncharacterized protein & S. italica & 3 & 37 & 0.05 & 7.27 & 55802 & TCA \\
\hline F2DGF3 & Predicted protein & H. vulgare & 13 & 167 & 0.04 & 6.81 & 115601 & TCA \\
\hline B5BLW2 & Carotenoid cleavage dioxygenase 1 & M. truncatula & 3 & 42 & 0.04 & 6.79 & 61063 & secondary metabolism \\
\hline A8MQH4 & Methylmalonate-semialdehyde dehydrogenase & A. thaliana & 2 & 69 & 0.04 & 8.76 & 65229 & amino acid metabolism \\
\hline K4AMY6 & Uncharacterized protein & S. italica & 11 & 183 & 0.04 & 5.91 & 66919 & C1-metabolism \\
\hline F2DIS4 & Malic enzyme & H. vulgare & 4 & 56 & 0.04 & 7.40 & 68411 & TCA \\
\hline K3YKV0 & Uncharacterized protein & S. italica & 12 & 79 & 0.03 & 5.54 & 87462 & cell \\
\hline Q38766 & Victorin binding protein & A. sativa & 5 & 70 & 0.03 & 6.96 & 112298 & photosynthesis \\
\hline B2KNE6 & Phospholipase D & H. annuиs & 9 & 142 & 0.03 & 5.52 & 92315 & lipid metabolism \\
\hline Q9XFX7 & Phospholipase D & C. plantagineum & 10 & 37 & 0.03 & 5.66 & 92228 & lipid metabolism \\
\hline Q9ZVY6 & $\mathrm{T} 25 \mathrm{~N} 20.17$ & A. thaliana & 5 & 53 & 0.03 & 5.58 & 90732 & protein \\
\hline J3MFV3 & Lipoxygenase & O. brachyantha & 11 & 47 & 0.02 & 6.77 & 103742 & hormone metabolism \\
\hline Q9ARB0 & Nbi-D protein & L. usitatissimum & 19 & 42 & 0.02 & 7.25 & 127688 & stress \\
\hline
\end{tabular}

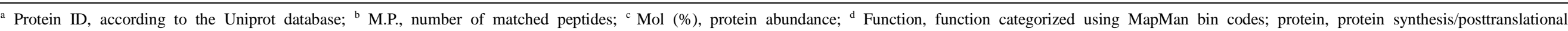

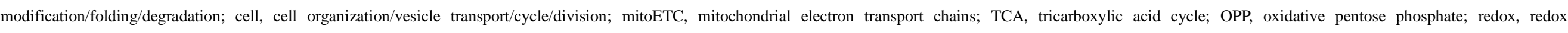
ascorbate/glutathione metabolism; RNA, RNA processing/regulation of transcription; and misc, miscellaneous. 
Supplemental Table 11. Proteins Identified in L. japonica Immature Flower Buds using Combinatorial Peptide Ligand Libraries Fractionation.

\begin{tabular}{|c|c|c|c|c|c|c|c|c|}
\hline No. & Protein $\mathrm{ID}^{\mathrm{a}}$ & Description & Species & M.P. ${ }^{b}$ & Score & $\mathrm{pI}$ & Mass (Da) & Function $^{\mathrm{c}}$ \\
\hline 1 & A0FKE6 & Chloroplast threonine deaminase 1 & S. lycopersicum & 9 & 173 & 6.37 & 66653 & amino acid metabolism \\
\hline 2 & A7WMM1 & Alanine:glyoxylate aminotransferase & L. caprifolium & 6 & 38 & 8.43 & 13805 & amino acid metabolism \\
\hline 3 & A8J6J6 & Acetyl-CoA acyltransferase & C. reinhardtii & 8 & 65 & 7.99 & 47670 & amino acid metabolism \\
\hline 4 & A8MQH4 & Methylmalonate-semialdehyde dehydrogenase [acylating] & A. thaliana & 2 & 90 & 8.76 & 65229 & amino acid metabolism \\
\hline 5 & A8MQP1 & Adenosylhomocysteinase 1 & A. thaliana & 35 & 283 & 5.16 & 36022 & amino acid metabolism \\
\hline 6 & А9РАK9 & Putative uncharacterized protein & P. trichocarpa & 11 & 198 & 6.89 & 50623 & amino acid metabolism \\
\hline 7 & A9RZ64 & Predicted protein & P. patens & 2 & 154 & 7.87 & 50746 & amino acid metabolism \\
\hline 8 & B2VQE0 & Methionine synthase & O. ramosa & 171 & 2629 & 6.40 & 84862 & amino acid metabolism \\
\hline 9 & B5LAV3 & Putative isopropylmalate synthase & C. аппиит & 5 & 119 & 6.54 & 66084 & amino acid metabolism \\
\hline 10 & B6THZ8 & Threonine synthase & Z. mays & 2 & 48 & 6.92 & 58091 & amino acid metabolism \\
\hline 11 & B9S7T6 & Transaminase mtnE, putative & R. communis & 7 & 71 & 7.28 & 50909 & amino acid metabolism \\
\hline 12 & C8YNG6 & 3-ketoacyl CoA thiolase 1 & P. hybrida & 10 & 96 & 8.29 & 49405 & amino acid metabolism \\
\hline 13 & D2KZ81 & S-adenosylmethionine synthase & M. domestica & 3 & 141 & 4.94 & 24831 & amino acid metabolism \\
\hline 14 & D7LW57 & Ketol-acid reductoisomerase & A. lyrata & 11 & 300 & 6.80 & 64346 & amino acid metabolism \\
\hline 15 & E1ZNM5 & Putative uncharacterized protein & C. variabilis & 8 & 47 & 6.83 & 60332 & amino acid metabolism \\
\hline 16 & E4MWC3 & Aspartate aminotransferase & T. halophila & 8 & 66 & 9.25 & 49678 & amino acid metabolism \\
\hline 17 & E5GCI7 & Ornithine carbamoyltransferase & C. melo & 3 & 123 & 8.40 & 42175 & amino acid metabolism \\
\hline 18 & F2CSU5 & Predicted protein & H. vulgare & 4 & 60 & 8.32 & 45209 & amino acid metabolism \\
\hline 19 & F2CYN2 & Predicted protein & H. vulgare & 52 & 794 & 6.18 & 84718 & amino acid metabolism \\
\hline 20 & F2DAD8 & Predicted protein & H. vulgare & 6 & 54 & 7.15 & 50084 & amino acid metabolism \\
\hline 21 & G5DWR0 & Branched-chain-amino-acid aminotransferase & S. latifolia & 4 & 33 & 5.83 & 38581 & amino acid metabolism \\
\hline 22 & G8HAB2 & PLP-dependent aminotransferase & P. somniferum & 8 & 144 & 5.63 & 53501 & amino acid metabolism \\
\hline 23 & H9C956 & 3-deoxy-D-arabino-heptulosonate 7-phosphate synthase & N. benthamiana & 5 & 98 & 8.28 & 53191 & amino acid metabolism \\
\hline 24 & K3YH65 & Uncharacterized protein & S. italica & 2 & 40 & 7.56 & 56313 & amino acid metabolism \\
\hline 25 & K3YH94 & Uncharacterized protein & S. italica & 14 & 195 & 5.99 & 56064 & amino acid metabolism \\
\hline 26 & K3YSB2 & Uncharacterized protein & S. italica & 21 & 133 & 8.63 & 50388 & amino acid metabolism \\
\hline 27 & K3ZSX0 & Uncharacterized protein & S. italica & 6 & 120 & 7.64 & 53564 & amino acid metabolism \\
\hline 28 & Q069K2 & 5-methyltetrahydropteroyltriglutamate--homocysteine methyltransferase & N. suaveolens & 72 & 857 & 6.62 & 85060 & amino acid metabolism \\
\hline 29 & Q42425 & Aspartate aminotransferase & P. miliaceum & 17 & 124 & 8.48 & 50477 & amino acid metabolism \\
\hline 30 & Q43057 & Aspartate aminotransferase & P. miliaceum & 6 & 49 & 7.33 & 48154 & amino acid metabolism \\
\hline 31 & Q4H1G1 & Adenosylhomocysteinase & B. vulgaris & 32 & 204 & 6.19 & 54052 & amino acid metabolism \\
\hline
\end{tabular}




\begin{tabular}{|c|c|c|c|c|c|c|c|c|}
\hline 32 & Q4H1G2 & Methionine synthase & B. vulgaris & 89 & 1267 & 6.46 & 88546 & amino acid metabolism \\
\hline 33 & Q5GAQ3 & Putative alanine aminotransferase & Z. mays & 11 & 163 & 5.64 & 57003 & amino acid metabolism \\
\hline 34 & Q84RD8 & Adenosylhomocysteinase & M. truncatula & 37 & 427 & 6.01 & 53790 & amino acid metabolism \\
\hline 35 & Q8LGJ6 & Phosphoglycerate dehydrogenase-like protein & A. thaliana & 6 & 116 & 6.76 & 63556 & amino acid metabolism \\
\hline 36 & Q9S768 & Alanine aminotransferase & O. sativa & 6 & 207 & 6.65 & 53130 & amino acid metabolism \\
\hline 37 & Q9SRV5 & 5-methyltetrahydropteroyltriglutamate--homocysteine methyltransferase & A. thaliana & 83 & 1029 & 6.51 & 84873 & amino acid metabolism \\
\hline 38 & F2CQP8 & Predicted protein & H. vulgare & 9 & 61 & 5.49 & 32811 & biodegradation of xenobiotics. \\
\hline 39 & F2DM28 & Predicted protein & H. vulgare & 8 & 84 & 7.33 & 38865 & biodegradation of xenobiotics. \\
\hline 40 & K3Z7G1 & Uncharacterized protein & S. italica & 11 & 130 & 6.60 & 39285 & biodegradation of xenobiotics. \\
\hline 41 & O04428 & Putative uncharacterized protein & C. paradisi & 15 & 236 & 5.59 & 32737 & biodegradation of xenobiotics. \\
\hline 42 & A6XMY8 & Methylenetetrahydrofolate reductase & T. monococcum & 13 & 146 & 6.20 & 65461 & C1-metabolism \\
\hline 43 & B6TN41 & Regulator of ribonuclease activity A & Z. mays & 4 & 63 & 6.18 & 18390 & C1-metabolism \\
\hline 44 & F2D3S8 & Serine hydroxymethyltransferase & H. vulgare & 20 & 77 & 8.21 & 56215 & C1-metabolism \\
\hline 45 & K3Z566 & Uncharacterized protein & S. italica & 29 & 401 & 7.77 & 59005 & C1-metabolism \\
\hline 46 & $\mathrm{O} 23254$ & Serine hydroxymethyltransferase & A. thaliana & 50 & 647 & 7.23 & 52141 & C1-metabolism \\
\hline 47 & Q8LBY1 & Hydroxymethyltransferase & A. thaliana & 19 & 457 & 7.02 & 52129 & C1-metabolism \\
\hline 48 & Q9SUU0 & Serine hydroxymethyltransferase & A. thaliana & 6 & 85 & 8.05 & 50988 & C1-metabolism \\
\hline 49 & A2Q5W0 & Alpha-tubulin & M. truncatula & 18 & 384 & 5.10 & 50457 & cell \\
\hline 50 & A4ZSZ4 & Actin & P. trichocarpa & 22 & 330 & 5.49 & 41913 & cell \\
\hline 51 & A5AW47 & Profilin & V. vinifera & 17 & 71 & 6.16 & 92852 & cell \\
\hline 52 & A7KQH1 & Beta-tubulin & E. grandis & 69 & 882 & 4.86 & 51031 & cell \\
\hline 53 & A7KQH2 & Beta-tubulin & E. grandis & 63 & 782 & 4.84 & 50255 & cell \\
\hline 54 & A8DSD0 & Alpha-tubulin 7 & P. tremuloides & 23 & 337 & 5.15 & 50382 & cell \\
\hline 55 & A8IM71 & Coatomer subunit gamma & C. reinhardtii & 4 & 38 & 5.29 & 97872 & cell \\
\hline 56 & В6TJ90 & Histone $\mathrm{H} 4$ & Z. mays & 9 & 119 & 9.67 & 20120 & cell \\
\hline 57 & B6ZK00 & Peroxisomal biogenesis factor 11 family protein & G. $\max$ & 2 & 110 & 9.76 & 26114 & cell \\
\hline 58 & D0ELH5 & Peptidyl-prolyl cis-trans isomerase & C. sinensis & 10 & 209 & 8.46 & 18310 & cell \\
\hline 59 & D6PAY2 & Peptidyl-prolyl cis-trans isomerase & V. hybrid cultivar & 15 & 217 & 8.46 & 18582 & cell \\
\hline 60 & E0D6S0 & Actin & G. bicolor & 63 & 1195 & 5.31 & 41913 & cell \\
\hline 61 & E1ZSN8 & Putative uncharacterized protein & C. variabilis & 5 & 55 & 5.14 & 39874 & cell \\
\hline 62 & E3NYI6 & Peptidyl-prolyl cis-trans isomerase & A. diogoi & 10 & 59 & 8.44 & 18332 & cell \\
\hline 63 & E4MW44 & mRNA, clone: RTFL01-05-I15 & T. halophila & 36 & 462 & 5.58 & 42078 & cell \\
\hline
\end{tabular}




\begin{tabular}{|c|c|c|c|c|c|c|c|c|}
\hline 64 & F2DDG9 & Predicted protein & H. vulgare & 2 & 38 & 5.66 & 52441 & cell \\
\hline 65 & F2E6F3 & Coatomer subunit gamma & H. vulgare & 4 & 165 & 5.17 & 100353 & cell \\
\hline 66 & F2E7L1 & Predicted protein & H. vulgare & 17 & 197 & 9.95 & 50919 & cell \\
\hline 67 & F2EL23 & Predicted protein & H. vulgare & 37 & 937 & 5.29 & 90854 & cell \\
\hline 68 & F8WL60 & Beta actin & R. stylosa & 35 & 774 & 5.27 & 33424 & cell \\
\hline 69 & G7L7D9 & Profilin & M. truncatula & 8 & 40 & 5.40 & 14401 & cell \\
\hline 70 & H9NIE1 & Cell division cycle protein 48 & C. sinensis & 29 & 551 & 5.29 & 90644 & cell \\
\hline 71 & K3XE87 & Uncharacterized protein & S. italica & 6 & 70 & 5.90 & 106127 & cell \\
\hline 72 & K3XFY9 & Uncharacterized protein & S. italica & 14 & 175 & 6.81 & 64010 & cell \\
\hline 73 & K3XZA2 & Uncharacterized protein & S. italica & 13 & 178 & 9.58 & 23221 & cell \\
\hline 74 & K3Y4M8 & Uncharacterized protein & S. italica & 6 & 151 & 5.39 & 194893 & cell \\
\hline 75 & K3YKV0 & Uncharacterized protein & S. italica & 8 & 117 & 5.54 & 87462 & cell \\
\hline 76 & K3Z913 & Uncharacterized protein & S. italica & 16 & 316 & 9.47 & 26793 & cell \\
\hline 77 & K4A4Z8 & Uncharacterized protein & S. italica & 7 & 40 & 6.96 & 137642 & cell \\
\hline 78 & K4A9V6 & Uncharacterized protein & S. italica & 17 & 193 & 5.10 & 50519 & cell \\
\hline 79 & Q06Z74 & Fibrillin 4 & C. canephora & 11 & 288 & 8.88 & 32064 & cell \\
\hline 80 & Q2TFP1 & Tubulin B4 & G. $\max$ & 35 & 465 & 4.83 & 51083 & cell \\
\hline 81 & Q39834 & Clathrin heavy chain & G. $\max$ & 8 & 72 & 5.55 & 194430 & cell \\
\hline 82 & Q52UN0 & Peptidyl-prolyl cis-trans isomerase & C. sativus & 13 & 52 & 8.44 & 18340 & cell \\
\hline 83 & Q9XEN8 & Annexin & N.tabacum & 5 & 44 & 5.53 & 36029 & cell \\
\hline 84 & Q9ZQT0 & Actin & G. echinata & 22 & 282 & 6.19 & 17082 & cell \\
\hline 85 & $\mathrm{~A} 2 \mathrm{PZC} 2$ & UDP-Glucose:protein transglucosylase & C. reinhardtii & 7 & 111 & 6.33 & 39846 & cell wall \\
\hline 86 & A5JVC6 & Putative uncharacterized protein & B. campestris & 7 & 142 & 6.25 & 34259 & cell wall \\
\hline 87 & A8MR50 & $60 \mathrm{~S}$ ribosomal protein $\mathrm{L} 34-1$ & A. thaliana & 2 & 107 & 11.84 & 11023 & cell wall \\
\hline 88 & $\mathrm{~B} 1 \mathrm{P} 2 \mathrm{~T} 2$ & Cellulose synthase-like CslF3 & H. vulgare & 24 & 34 & 7.74 & 96260 & cell wall \\
\hline 89 & B6T9G3 & Alpha-1,4-glucan-protein synthase 1 & Z. mays & 34 & 411 & 7.05 & 41278 & cell wall \\
\hline 90 & B9GXZ8 & Pectinesterase & P. trichocarpa & 6 & 77 & 8.98 & 63820 & cell wall \\
\hline 91 & E5F725 & Putative uncharacterized protein & E. parvulum & 6 & 255 & 7.11 & 42041 & cell wall \\
\hline 92 & F2CVG4 & Predicted protein & H. vulgare & 27 & 371 & 6.73 & 76549 & cell wall \\
\hline 93 & F6HGZ1 & Pectinesterase & V. vinifera & 6 & 74 & 7.49 & 63329 & cell wall \\
\hline 94 & F6HXK8 & Pectinesterase & V. vinifera & 6 & 92 & 8.54 & 69782 & cell wall \\
\hline 95 & G5DXR5 & UDP-glucuronic acid decarboxylase & S. latifolia & 15 & 323 & 7.27 & 38756 & cell wall \\
\hline
\end{tabular}




\begin{tabular}{|c|c|c|c|c|c|c|c|c|}
\hline 96 & J9Z414 & B.-D-glucan exohydolase & E. guineensis & 4 & 111 & 7.88 & 69462 & cell wall \\
\hline 97 & Q1EMR1 & Nucleoside-diphopshate-sugar dehydratase & P. major & 7 & 69 & 5.17 & 22710 & cell wall \\
\hline 98 & Q5ZF83 & Putative uncharacterized protein & P. major & 15 & 120 & 7.28 & 25390 & cell wall \\
\hline 99 & Q8GZD5 & Xyloglucan endotransglycosylase & P. tremula & 39 & 430 & 7.75 & 34481 & cell wall \\
\hline 100 & Q8LDN8 & At1g63180 & A. thaliana & 8 & 63 & 6.73 & 39171 & cell wall \\
\hline 101 & Q9FZG9 & Putative pectinesterase & A. thaliana & 19 & 111 & 9.45 & 60580 & cell wall \\
\hline 102 & Q9LF33 & UDP-glucose 6-dehydrogenase & A. thaliana & 15 & 466 & 6.04 & 53653 & cell wall \\
\hline 103 & Q9LLB8 & Exoglucanase & Z. mays & 9 & 138 & 7.34 & 67356 & cell wall \\
\hline 104 & Q9SN95 & AT3G46440 protein & A. thaliana & 20 & 344 & 7.52 & 38593 & cell wall \\
\hline 105 & Q9ZV36 & Putative nucleotide-sugar dehydratase & A. thaliana & 15 & 245 & 8.46 & 38768 & cell wall \\
\hline 106 & G5DWK9 & 3-dehydroquinate synthase & S. latifolia & 3 & 37 & 7.03 & 13075 & co-factor and vitamine metabolism \\
\hline 107 & K3ZUR5 & Uncharacterized protein & S. italica & 22 & 758 & 6.24 & 36749 & co-factor and vitamine metabolism \\
\hline 108 & Q6QND3 & Putative pyridoxine biosynthesis protein isoform A & N. tabacum & 6 & 122 & 6.32 & 33353 & co-factor and vitamine metabolism \\
\hline 109 & $\mathrm{~A} 2 \mathrm{~V} 884$ & Translationally controlled tumor protein like protein & N. tabacum & 4 & 46 & 4.53 & 18052 & development \\
\hline 110 & B4FL17 & Translationally-controlled tumor protein & Z. mays & 16 & 123 & 4.67 & 18773 & development \\
\hline 111 & E4MWY0 & mRNA, clone: RTFL01-19-L03 & T. halophila & 19 & 102 & 9.39 & 120492 & development \\
\hline 112 & F2DWM5 & Predicted protein & H. vulgare & 13 & 134 & 9.22 & 92351 & development \\
\hline 113 & I6LE48 & Uncharacterized protein & D. excelsa & 4 & 48 & 5.03 & 33659 & development \\
\hline 114 & Q2LFC3 & AGO1-2 & N. benthamiana & 19 & 261 & 9.31 & 109989 & development \\
\hline 115 & Q2LFC4 & AGO1-1 & N. benthamiana & 19 & 154 & 9.45 & 118109 & development \\
\hline 116 & A8JJQ6 & Histone $\mathrm{H} 2 \mathrm{~B}$ & C. reinhardtii & 8 & 70 & 9.85 & 16544 & DNA \\
\hline 117 & E1Z839 & Putative uncharacterized protein & C. variabilis & 11 & 38 & 6.28 & 111491 & DNA \\
\hline 118 & $\mathrm{~A} 5 \mathrm{C} 113$ & Putative uncharacterized protein & V. vinifera & 4 & 90 & 5.59 & 17365 & fermentation \\
\hline 119 & A8II37 & Predicted protein & C. reinhardtii & 11 & 86 & 6.52 & 101172 & fermentation \\
\hline 120 & B6T715 & Aldehyde dehydrogenase & Z. mays & 3 & 80 & 6.76 & 59108 & fermentation \\
\hline 121 & K3ZR86 & Uncharacterized protein & S. italica & 6 & 35 & 9.01 & 74682 & fermentation \\
\hline 122 & Q42953 & Alcohol dehydrogenase & N.tabacum & 8 & 54 & 7.05 & 41981 & fermentation \\
\hline 123 & B0M1B8 & Citrate synthase & G. $\max$ & 4 & 55 & 8.09 & 54427 & gluconeogenesis \\
\hline 124 & O48903 & Malate dehydrogenase & M. sativa & 31 & 780 & 8.29 & 38397 & gluconeogenesis \\
\hline 125 & Q645M9 & Glyoxisomal malate dehydrogenase & S. lycopersicum & 17 & 395 & 7.94 & 38007 & gluconeogenesis \\
\hline 126 & A3QVW8 & Glucose-6-phosphate isomerase & M. polymorpha & 10 & 68 & 7.18 & 68171 & glycolysis \\
\hline 127 & A8ASG2 & Phosphoenolpyruvate carboxylase & A. arborescens & 21 & 226 & 6.46 & 110813 & glycolysis \\
\hline
\end{tabular}




\begin{tabular}{|c|c|c|c|c|c|c|c|c|}
\hline 128 & B3TLL4 & Triosephosphate isomerase & E. guineensis & 24 & 247 & 6.23 & 27476 & glycolysis \\
\hline 129 & B4FNW1 & Triosephosphate isomerase & Z. mays & 28 & 344 & 5.68 & 27236 & glycolysis \\
\hline 130 & B4FQM2 & Pyrophosphate--fructose 6-phosphate 1-phosphotransferase B. subunit & Z. mays & 7 & 110 & 6.35 & 61607 & glycolysis \\
\hline 131 & B4G0K4 & Phosphoglycerate kinase & Z. mays & 38 & 569 & 5.87 & 42470 & glycolysis \\
\hline 132 & B6SJZ8 & Glyceraldehyde-3-phosphate dehydrogenase, cytosolic & Z. mays & 100 & 2845 & 6.96 & 36614 & glycolysis \\
\hline 133 & B6SWT4 & Glucose-6-phosphate isomerase & Z. mays & 8 & 273 & 5.99 & 67980 & glycolysis \\
\hline 134 & В6Т3Р9 & Enolase & Z. mays & 10 & 352 & 5.82 & 48386 & glycolysis \\
\hline 135 & B6TGG2 & 2,3-bisphosphoglycerate-independent phosphoglycerate mutase & Z. mays & 15 & 174 & 5.53 & 60811 & glycolysis \\
\hline 136 & B6TGL7 & Enolase & Z. mays & 14 & 502 & 6.23 & 50512 & glycolysis \\
\hline 137 & C4B8E5 & Glyceraldehyde-3-phosphate dehydrogenase & T. gesneriana & 144 & 4605 & 7.96 & 20964 & glycolysis \\
\hline 138 & D7NHW9 & 2-phospho-D-glycerate hydrolase & P. trifoliata & 61 & 1595 & 5.78 & 47986 & glycolysis \\
\hline 139 & E0D6S1 & Glyceraldehyde 3-phosphate dehydrogenase & G. bicolor & 61 & 1472 & 6.13 & 36957 & glycolysis \\
\hline 140 & E4MWY6 & Glucose-6-phosphate isomerase & T. halophila & 8 & 233 & 5.60 & 67312 & glycolysis \\
\hline 141 & E4MX61 & mRNA, clone: RTFL01-20-P01 & T. halophila & 10 & 72 & 6.84 & 53948 & glycolysis \\
\hline 142 & E4MY36 & mRNA, clone: RTFL01-37-B16 & T. halophila & 10 & 80 & 6.25 & 63555 & glycolysis \\
\hline 143 & E5GBV8 & Glyceraldehyde-3-phosphate dehydrogenase & C. melo & 109 & 2853 & 7.12 & 36754 & glycolysis \\
\hline 144 & E5GBV9 & Glyceraldehyde-3-phosphate dehydrogenase & C. melo & 149 & 2823 & 6.62 & 36471 & glycolysis \\
\hline 145 & E5GBW6 & $26 \mathrm{~S}$ proteasome non-ATPase regulatory subunit & C. melo & 2 & 76 & 5.92 & 44456 & glycolysis \\
\hline 146 & F2CR08 & Predicted protein & H. vulgare & 45 & 870 & 5.55 & 48427 & glycolysis \\
\hline 147 & F2CS51 & Pyruvate kinase & H. vulgare & 7 & 23 & 7.56 & 56102 & glycolysis \\
\hline 148 & $\mathrm{~F} 2 \mathrm{CW} 31$ & Predicted protein & H. vulgare & 14 & 205 & 6.21 & 63075 & glycolysis \\
\hline 149 & F2D6I8 & Predicted protein & H. vulgare & 67 & 1731 & 7.14 & 36874 & glycolysis \\
\hline 150 & F2EAT2 & Pyruvate kinase & H. vulgare & 2 & 40 & 6.35 & 55816 & glycolysis \\
\hline 151 & G9MA91 & Fructose-bisphosphate aldolase & L. grandiflorum & 8 & 266 & 6.28 & 37690 & glycolysis \\
\hline 152 & J3RTS9 & Glyceraldehyde-3-phosphate dehydrogenase & C. pentagona & 166 & 4476 & 7.23 & 32382 & glycolysis \\
\hline 153 & K3XFX7 & Uncharacterized protein & S. italica & 2 & 96 & 6.68 & 64026 & glycolysis \\
\hline 154 & K3XL57 & Uncharacterized protein & S. italica & 21 & 171 & 5.48 & 27435 & glycolysis \\
\hline 155 & K3XWI6 & Uncharacterized protein & S. italica & 10 & 162 & 6.60 & 55489 & glycolysis \\
\hline 156 & K3XXC5 & Uncharacterized protein & S. italica & 59 & 577 & 7.06 & 42409 & glycolysis \\
\hline 157 & K3Y5R7 & Uncharacterized protein & S. italica & 14 & 93 & 9.55 & 73000 & glycolysis \\
\hline 158 & K3Z5E2 & Uncharacterized protein & S. italica & 5 & 110 & 8.09 & 56837 & glycolysis \\
\hline 159 & K4A6V8 & Uncharacterized protein & S. italica & 13 & 138 & 7.03 & 70549 & glycolysis \\
\hline
\end{tabular}




\begin{tabular}{|c|c|c|c|c|c|c|c|c|}
\hline 160 & O04975 & Fructose-bisphosphate aldolase & M. crystalL. & 12 & 182 & 6.92 & 38362 & glycolysis \\
\hline 161 & Q19TV8 & UDP-glucose pyrophosphorylase & C. melo & 29 & 455 & 7.17 & 52201 & glycolysis \\
\hline 162 & Q110X5 & Pyruvate kinase & C. аппиит & 10 & 44 & 6.84 & 56114 & glycolysis \\
\hline 163 & Q1WFH5 & Phosphoenolpyruvate carboxylase isoform 3 & C. hilariana & 18 & 125 & 8.94 & 41462 & glycolysis \\
\hline 164 & Q6PKU2 & Putative glyceraldehyde-3-phosphate dehydrogenase & O. minor & 91 & 2316 & 7.09 & 22122 & glycolysis \\
\hline 165 & Q6T379 & Triosephosphate isomerase & S. chacoense & 23 & 470 & 5.99 & 27251 & glycolysis \\
\hline 166 & Q8H945 & Phosphoenolpyruvate carboxylase & L. japonicus & 15 & 206 & 5.88 & 111020 & glycolysis \\
\hline 167 & Q944T3 & Glyceraldehyde 3-phosphate dehydrogenase 2 & F. ananassa & 81 & 2694 & 9.20 & 13954 & glycolysis \\
\hline 168 & Q9FX54 & At1g13440/F13B4_8 & A. thaliana & 132 & 3915 & 7.18 & 37004 & glycolysis \\
\hline 169 & B6T6Q5 & GAST1 protein & Z. mays & 8 & 35 & 9.10 & 12384 & hormone metabolism \\
\hline 170 & C4NZX3 & Lipoxygenase & C. sinensis & 14 & 165 & 6.83 & 102365 & hormone metabolism \\
\hline 171 & E3WHD5 & Lipoxygenase & V. hybrid cultivar & 15 & 279 & 6.55 & 100738 & hormone metabolism \\
\hline 172 & F2DLJ7 & Predicted protein & H. vulgare & 2 & 143 & 7.77 & 44423 & hormone metabolism \\
\hline 173 & F2DP49 & Predicted protein & H. vulgare & 12 & 116 & 8.63 & 25913 & hormone metabolism \\
\hline 174 & F6HZ11 & Lipoxygenase & V. vinifera & 5 & 74 & 6.04 & 99463 & hormone metabolism \\
\hline 175 & I1LJS6 & Lipoxygenase & G. $\max$ & 14 & 304 & 7.36 & 104140 & hormone metabolism \\
\hline 176 & J3MFV3 & Lipoxygenase & O. brachyantha & 11 & 35 & 6.77 & 103742 & hormone metabolism \\
\hline 177 & K3XHS0 & Uncharacterized protein & S. italica & 2 & 37 & 5.81 & 48047 & hormone metabolism \\
\hline 178 & P93698 & Lipoxygenase & V. unguiculata & 13 & 179 & 6.49 & 102731 & hormone metabolism \\
\hline 179 & Q0VYL5 & GASA & F. sylvatica & 2 & 44 & 9.03 & 12634 & hormone metabolism \\
\hline 180 & Q4ZJ73 & 12-oxophytodienoate reductase & H. brasiliensis & 5 & 34 & 8.72 & 44571 & hormone metabolism \\
\hline 181 & B2KNE6 & Phospholipase D & H. annuus & 8 & 142 & 5.52 & 92315 & lipid metabolism \\
\hline 182 & B5LAU5 & DH putative B.-hydroxyacyl-ACP dehydratase & C. аппиит & 8 & 78 & 9.29 & 24080 & lipid metabolism \\
\hline 183 & B6SXV4 & Peroxisomal fatty acid beta-oxidation multifunctional protein & Z. mays & 5 & 79 & 9.17 & 79104 & lipid metabolism \\
\hline 184 & E4MVK9 & mRNA, clone: RTFL01-02-O16 & T. halophila & 3 & 128 & 8.41 & 92372 & lipid metabolism \\
\hline 185 & F2CTK4 & Predicted protein & H. vulgare & 16 & 65 & 9.04 & 83843 & lipid metabolism \\
\hline 186 & F2DWX8 & Predicted protein & H. vulgare & 2 & 27 & 6.39 & 21692 & lipid metabolism \\
\hline 187 & F2EG33 & Predicted protein & H. vulgare & 9 & 92 & 8.76 & 52947 & lipid metabolism \\
\hline 188 & F2WMV4 & Acetyl-CoA carboxylase BC subunit & J. curcas & 6 & 76 & 7.71 & 58747 & lipid metabolism \\
\hline 189 & G1DVA7 & Non-specific lipid-transfer protein & H. annuиs & 4 & 42 & 8.41 & 12193 & lipid metabolism \\
\hline 190 & H9A1W2 & Acyl-activating enzyme 10 & C. sativa & 9 & 150 & 6.49 & 62013 & lipid metabolism \\
\hline 191 & O04946 & Enoyl-ACP reductase & N. tabacum & 12 & 159 & 8.78 & 41925 & lipid metabolism \\
\hline
\end{tabular}




\begin{tabular}{|c|c|c|c|c|c|c|c|c|}
\hline 192 & O81830 & Putative uncharacterized protein AT4g27270 & A. thaliana & 9 & 223 & 6.79 & 22355 & lipid metabolism \\
\hline 193 & Q42793 & Acetyl CoA carboxylase & G. $\max$ & 5 & 46 & 6.32 & 253225 & lipid metabolism \\
\hline 194 & Q43248 & Acetyl CoA carboxylase & Z. mays & 8 & 37 & 6.28 & 188959 & lipid metabolism \\
\hline 195 & Q9LLR1 & Acetyl-CoA carboxylase & G. $\max$ & 10 & 223 & 7.50 & 59306 & lipid metabolism \\
\hline 196 & Q9SQI8 & 2-oxoacid dehydrogenases acyltransferase family protein & A. thaliana & 9 & 154 & 8.37 & 50106 & lipid metabolism \\
\hline 197 & Q9XFX7 & Phospholipase D & C. plantagineum & 7 & 35 & 5.66 & 92228 & lipid metabolism \\
\hline 198 & Q9XFX8 & Phospholipase D & C. plantagineum & 3 & 99 & 5.44 & 92155 & lipid metabolism \\
\hline 199 & A3QQY2 & Sucrose synthase & C. intybus & 41 & 363 & 6.39 & 92338 & major $\mathrm{CHO}$ metabolism \\
\hline 200 & B3F8H6 & Sucrose synthase & N. langsdorffii & 36 & 453 & 6.40 & 92814 & major $\mathrm{CHO}$ metabolism \\
\hline 201 & В6ТВ29 & Fructokinase-2 & Z. mays & 14 & 129 & 5.58 & 35875 & major $\mathrm{CHO}$ metabolism \\
\hline 202 & F2E397 & Predicted protein & H. vulgare & 10 & 262 & 5.87 & 34933 & major $\mathrm{CHO}$ metabolism \\
\hline 203 & G3JZV4 & Sucrose synthase & O. ramosa & 40 & 562 & 6.74 & 92420 & major $\mathrm{CHO}$ metabolism \\
\hline 204 & Q1HG95 & Sucrose synthase & V. album & 15 & 50 & 6.09 & 92607 & major $\mathrm{CHO}$ metabolism \\
\hline 205 & Q5BLY1 & Plastid alpha-amylase & A. chinensis & 8 & 59 & 6.30 & 101618 & major $\mathrm{CHO}$ metabolism \\
\hline 206 & Q6VWJ5 & Fructokinase 3 & S. lycopersicum & 14 & 221 & 5.80 & 41803 & major $\mathrm{CHO}$ metabolism \\
\hline 207 & Q7XAE2 & Putative fructokinase 2 & P. integrifolia & 33 & 477 & 5.35 & 35181 & major $\mathrm{CHO}$ metabolism \\
\hline 208 & K3XHU5 & Uncharacterized protein & S. italica & 15 & 228 & 6.43 & 48590 & metal handling \\
\hline 209 & A4UMS1 & Myo-inositol-1-phosphate synthase isoform 1 & X. viscosa & 27 & 479 & 5.41 & 56899 & minor CHO metabolism \\
\hline 210 & A2JGX1 & Beta-galactosidase & S. lycopersicum & 4 & 51 & 8.09 & 98181 & misc \\
\hline 211 & A8MRF3 & 60S ribosomal protein L18a-1 & A. thaliana & 3 & 77 & 9.73 & 36337 & misc \\
\hline 212 & B0M183 & Acetylcholinesterase & M. atropurpureum & 4 & 117 & 6.77 & 43320 & misc \\
\hline 213 & B3TLW0 & Glutathione S-transferase & E. guineensis & 3 & 43 & 5.76 & 27569 & misc \\
\hline 214 & B6SUH6 & Non-cyanogenic B.-glucosidase & Z. mays & 27 & 66 & 5.80 & 56909 & misc \\
\hline 215 & B6T6Q8 & Alcohol dehydrogenase class 3 & Z. mays & 8 & 143 & 7.02 & 41639 & misc \\
\hline 216 & B9HJ23 & Predicted protein & P. trichocarpa & 12 & 179 & 6.54 & 101700 & misc \\
\hline 217 & E5GBJ6 & Alcohol dehydrogenase & C. melo & 3 & 131 & 8.47 & 40658 & misc \\
\hline 218 & F2CRY1 & Predicted protein & H. vulgare & 3 & 34 & 8.70 & 69011 & misc \\
\hline 219 & F2CUK9 & Predicted protein & H. vulgare & 15 & 27 & 8.82 & 31275 & misc \\
\hline 220 & F2CYA6 & Predicted protein & H. vulgare & 93 & 1319 & 6.06 & 17119 & misc \\
\hline 221 & Q6L619 & B.-galactosidase & $R$. sativus & 6 & 101 & 8.38 & 93664 & misc \\
\hline 222 & Q9LLA9 & Fatty acid hydroperoxide lyase & S. lycopersicum & 3 & 124 & 6.81 & 53737 & misc \\
\hline 223 & Q9ZVV4 & At1g55570/T5A14_1 & A. thaliana & 4 & 32 & 8.91 & 62687 & misc \\
\hline
\end{tabular}




\begin{tabular}{|c|c|c|c|c|c|c|c|c|}
\hline 224 & B5TM95 & NADH dehydrogenase subunit 7 & A. thaliana & 10 & 202 & 7.12 & 45555 & mitoETC \\
\hline 225 & C6GFP3 & ATP synthase subunit B. & G. hirsutum & 148 & 2953 & 6.29 & 59987 & mitoETC \\
\hline 226 & J7MPY1 & ATP synthase subunit alpha & M. domestica & 18 & 125 & 6.61 & 55600 & mitoETC \\
\hline 227 & K3ZS26 & Uncharacterized protein & S. italica & 4 & 33 & 8.02 & 60854 & mitoETC \\
\hline 228 & Q2F951 & NADH dehydrogenase subunit 9 & O. sativa & 7 & 29 & 7.82 & 22694 & mitoETC \\
\hline 229 & Q41534 & ATP synthase subunit B. & T. aestivum & 132 & 2910 & 5.86 & 59326 & mitoETC \\
\hline 230 & Q5IA96 & Cytochrome oxidase subunit II & A. trichopoda & 2 & 91 & 4.75 & 9794 & mitoETC \\
\hline 231 & Q6R9J5 & ATPase subunit 4 & Z. mays & 4 & 39 & 8.48 & 24941 & mitoETC \\
\hline 232 & Q9SAQ0 & ATP synthase subunit beta & N. sylvestris & 74 & 1333 & 6.06 & 59597 & mitoETC \\
\hline 233 & F2D5T2 & Predicted protein & H. vulgare & 3 & 110 & 8.09 & 27859 & mitoETC \\
\hline 234 & Q34691 & ATP synthase subunit alpha & H. annuus & 22 & 314 & 6.60 & 55766 & mitoETC \\
\hline 235 & B5LAU9 & Glutamine synthetase & C. аппиит & 22 & 152 & 6.93 & 47926 & $\mathrm{~N}$-metabolism \\
\hline 236 & F2E3I7 & Glutamine synthetase & H. vulgare & 27 & 266 & 5.48 & 39331 & $\mathrm{~N}$-metabolism \\
\hline 237 & $\mathrm{O} 22331$ & Glutamine synthetase & H. brasiliensis & 15 & 309 & 6.20 & 39504 & $\mathrm{~N}$-metabolism \\
\hline 238 & B4FH62 & NAD-dependent epimerase/dehydratase & Z. mays & 7 & 68 & 9.04 & 31917 & not assigned \\
\hline 239 & B4FRS3 & Uncharacterized protein & Z. mays & 3 & 31 & 8.06 & 22134 & not assigned \\
\hline 240 & B6SVI5 & Putative uncharacterized protein & Z. mays & 8 & 32 & 8.67 & 24337 & not assigned \\
\hline 241 & B9H5E3 & Predicted protein & P. trichocarpa & 15 & 98 & 9.01 & 61685 & not assigned \\
\hline 242 & B9RI89 & Serine-threonine protein kinase, plant-type, putative & R. communis & 4 & 107 & 8.18 & 40375 & not assigned \\
\hline 243 & E5GCD6 & Multicopper oxidase & C. melo & 29 & 135 & 9.32 & 60810 & not assigned \\
\hline 244 & F2CT97 & Predicted protein & H. vulgare & 2 & 43 & 8.51 & 30094 & not assigned \\
\hline 245 & F2D642 & Predicted protein & H. vulgare & 5 & 40 & 8.72 & 44355 & not assigned \\
\hline 246 & F2DAC4 & Predicted protein & H. vulgare & 3 & 73 & 7.18 & 12136 & not assigned \\
\hline 247 & F6H3T7 & Putative uncharacterized protein & V. vinifera & 16 & 85 & 6.11 & 40651 & not assigned \\
\hline 248 & K3Z3S8 & Uncharacterized protein & S. italica & 4 & 37 & 6.64 & 95959 & not assigned \\
\hline 249 & Q04130 & G.-rich protein & S. lycopersicum & 2 & 32 & 9.98 & 7327 & not assigned \\
\hline 250 & Q8VWP8 & Acyltransferase-like protein & G. hirsutum & 3 & 103 & 5.96 & 48286 & not assigned \\
\hline 251 & Q9C6U3 & Putative uncharacterized protein T8G24.2 & A. thaliana & 6 & 120 & 7.59 & 34907 & not assigned \\
\hline 252 & Q9SA89 & FAD-binding and BBE domain-containing protein & A. thaliana & 8 & 38 & 8.60 & 60060 & not assigned \\
\hline 253 & Q9SIR5 & Expressed protein & A. thaliana & 4 & 42 & 6.84 & 32851 & not assigned \\
\hline 254 & Q9SP13 & Nucleoside diphosphate kinase & P. sativum & 8 & 66 & 9.32 & 25362 & not assigned \\
\hline 255 & F2DDZ1 & Predicted protein & H. vulgare & 2 & 116 & 6.42 & 46133 & nucleotide metabolism \\
\hline
\end{tabular}




\begin{tabular}{|c|c|c|c|c|c|c|c|c|}
\hline 256 & F2DTH4 & Adenylate kinase & H. vulgare & 5 & 99 & 7.81 & 26477 & nucleotide metabolism \\
\hline 257 & K4A938 & Uncharacterized protein & S. italica & 2 & 55 & 6.79 & 53547 & nucleotide metabolism \\
\hline 258 & A1BQW9 & Transketolase & N. attenuata & 28 & 170 & 6.80 & 32653 & OPP \\
\hline 259 & D6PPS8 & AT3G02360-like protein & C. grandiflora & 9 & 161 & 9.22 & 21010 & OPP \\
\hline 260 & D6PPS9 & AT3G02360-like protein & C. grandiflora & 12 & 177 & 9.22 & 21022 & OPP \\
\hline 261 & F2Z9R1 & Glucose-6-phosphate 1-dehydrogenase & N. benthamiana & 12 & 68 & 6.49 & 58894 & OPP \\
\hline 262 & K3XH89 & Uncharacterized protein & S. italica & 16 & 129 & 5.87 & 52978 & OPP \\
\hline 263 & O78327 & Transketolase 1 & C. аппиит & 38 & 338 & 6.62 & 80398 & OPP \\
\hline 264 & Q1KUX5 & 6-phosphogluconate dehydrogenase, decarboxylating & C. spinosa & 22 & 184 & 6.06 & 53777 & OPP \\
\hline 265 & Q7XJH9 & Transaldolase & S. lycopersicum & 17 & 198 & 5.96 & 48062 & OPP \\
\hline 266 & Q8LDR5 & Obg-like ATPase 1 & A. thaliana & 9 & 46 & 6.81 & 44776 & OPP \\
\hline 267 & Q9SH69 & 6-phosphogluconate dehydrogenase, decarboxylating & A. thaliana & 14 & 246 & 5.45 & 53686 & OPP \\
\hline 268 & A1YUL9 & Importin subunit alpha & N. benthamiana & 16 & 345 & 5.38 & 59114 & protein \\
\hline 269 & А2PYH3 & Alpha chain of nascent polypeptide associated complex & N. benthamiana & 4 & 225 & 4.50 & 21911 & protein \\
\hline 270 & A2Y926 & Proteasome subunit beta type & O. sativa & 2 & 63 & 5.48 & 26357 & protein \\
\hline 271 & A8I1Q9 & Ser/thr protein kinase & C. reinhardtii & 3 & 68 & 7.14 & 33293 & protein \\
\hline 272 & A8J1X8 & Aspartyl-tRNA synthetase & C. reinhardtii & 3 & 45 & 5.83 & 60686 & protein \\
\hline 273 & A8J597 & Ribosomal protein L12 & C. reinhardtii & 11 & 125 & 9.06 & 17822 & protein \\
\hline 274 & A8JCE1 & Predicted protein & C. reinhardtii & 17 & 36 & 7.94 & 33842 & protein \\
\hline 275 & A8MQA1 & $60 \mathrm{~S}$ ribosomal protein $\mathrm{L} 13$ & A. thaliana & 3 & 114 & 11.00 & 23525 & protein \\
\hline 276 & A8MQR4 & $60 \mathrm{~S}$ acidic ribosomal protein $\mathrm{P} 0-2$ & A. thaliana & 10 & 170 & 4.84 & 30656 & protein \\
\hline 277 & A8MR12 & $26 \mathrm{~S}$ proteasome non-ATPase regulatory subunit 14 & A. thaliana & 6 & 75 & 6.54 & 29246 & protein \\
\hline 278 & A8MRH4 & $60 \mathrm{~S}$ ribosomal protein $\mathrm{L} 7-2$ & A. thaliana & 5 & 40 & 10.10 & 21540 & protein \\
\hline 279 & A8MSE8 & Elongation factor 1-alpha & A. thaliana & 44 & 490 & 8.34 & 44670 & protein \\
\hline 280 & A9PD17 & Predicted protein & P. trichocarpa & 39 & 1072 & 5.82 & 47103 & protein \\
\hline 281 & A9PE52 & Gamma class glutathione transferase EF1Bgamma2 & P. trichocarpa & 7 & 141 & 5.80 & 48127 & protein \\
\hline 282 & В3H621 & Peptidase M1 family protein & A. thaliana & 5 & 121 & 6.55 & 106723 & protein \\
\hline 283 & B3H7J6 & 40S ribosomal protein S9-1 & A. thaliana & 27 & 202 & 9.80 & 19090 & protein \\
\hline 284 & B3TLL5 & 40S ribosomal protein $\mathrm{S} 19$ & E. guineensis & 13 & 58 & 10.04 & 16202 & protein \\
\hline 285 & B3TLN9 & Cytoplasmic ribosomal protein S15a & E. guineensis & 8 & 82 & 9.89 & 14862 & protein \\
\hline 286 & B3TLP3 & Translation elongation factor EF-1 B. chain & E. guineensis & 6 & 179 & 4.69 & 24523 & protein \\
\hline 287 & B3TLP4 & Ribosomal protein L30 & E. guineensis & 7 & 68 & 9.72 & 12451 & protein \\
\hline
\end{tabular}




\begin{tabular}{|c|c|c|c|c|c|c|c|c|}
\hline 288 & B3TLP8 & Ubiquitin extension protein-like protein & E. guineensis & 92 & 1286 & 9.79 & 17902 & protein \\
\hline 289 & B3TLQ5 & $60 \mathrm{~S}$ ribosomal protein $\mathrm{L} 11$ & E. guineensis & 6 & 154 & 9.92 & 20950 & protein \\
\hline 290 & B3TLV4 & $60 \mathrm{~S}$ ribosomal protein $\mathrm{L} 27$ & E. guineensis & 4 & 38 & 10.40 & 15833 & protein \\
\hline 291 & B3TLY3 & Ribosomal protein L15 & E. guineensis & 2 & 64 & 11.49 & 24371 & protein \\
\hline 292 & B3TM07 & Ribosomal protein L17 & E. guineensis & 11 & 36 & 10.49 & 20744 & protein \\
\hline 293 & В3ТМ23 & 40S ribosomal protein S4 & E. guineensis & 20 & 125 & 10.24 & 30023 & protein \\
\hline 294 & В3TM36 & $40 \mathrm{~S}$ ribosomal protein $\mathrm{S} 8$ & E. guineensis & 8 & 227 & 10.43 & 25347 & protein \\
\hline 295 & В3ТМ39 & Ribosomal protein S14 & E. guineensis & 6 & 89 & 10.70 & 16428 & protein \\
\hline 296 & B6T1W9 & $60 \mathrm{~S}$ ribosomal protein L12 & Z. mays & 9 & 152 & 9.85 & 17770 & protein \\
\hline 297 & B6TGB2 & Elongation factor 1-gamma 3 & Z. mays & 11 & 75 & 6.37 & 47357 & protein \\
\hline 298 & B6THG9 & $60 \mathrm{~S}$ ribosomal protein $\mathrm{L} 5-1$ & Z. mays & 10 & 183 & 9.32 & 34462 & protein \\
\hline 299 & B6TLT1 & Serine/threonine-protein phosphatase $2 \mathrm{~A} 65 \mathrm{kDa}$ regulatory subunit $\mathrm{AB}$. isoform & Z. mays & 4 & 43 & 5.03 & 66318 & protein \\
\hline 300 & B6TNX7 & Proteasome subunit alpha type & Z. mays & 5 & 147 & 5.40 & 30582 & protein \\
\hline 301 & B6TWN7 & Elongation factor 1-alpha & Z. mays & 21 & 72 & 9.07 & 49594 & protein \\
\hline 302 & B6UGQ7 & $60 \mathrm{~S}$ ribosomal protein $\mathrm{L} 23$ & Z. mays & 19 & 405 & 10.39 & 15232 & protein \\
\hline 303 & B9N744 & Gamma class glutathione transferase EF1Bgamma3 & P. trichocarpa & 18 & 300 & 6.05 & 48353 & protein \\
\hline 304 & C5WQZ5 & Putative uncharacterized protein Sb01g041210 & S. bicolor & 3 & 117 & 7.65 & 51802 & protein \\
\hline 305 & $\mathrm{D} 2 \mathrm{X} 5 \mathrm{~K} 2$ & DEAD-box RNA helicase-like protein & P. persica & 27 & 772 & 5.69 & 47191 & protein \\
\hline 306 & D7UPN0 & SEC13 family protein & L. japonicus & 3 & 71 & 6.01 & 32940 & protein \\
\hline 307 & E1Z4U0 & Putative uncharacterized protein & C. variabilis & 15 & 165 & 6.95 & 60152 & protein \\
\hline 308 & E1Z980 & Putative uncharacterized protein & C. variabilis & 3 & 54 & 6.49 & 44716 & protein \\
\hline 309 & E1Z9U8 & Putative uncharacterized protein & C. variabilis & 2 & 102 & 5.67 & 27282 & protein \\
\hline 310 & E1ZL24 & Putative uncharacterized protein & C. variabilis & 39 & 398 & 6.93 & 20995 & protein \\
\hline 311 & E4MVL4 & mRNA, clone: RTFL01-01-E20 & T. halophila & 7 & 126 & 5.58 & 54789 & protein \\
\hline 312 & E4MVY3 & mRNA, clone: RTFL01-01-M18 & T. halophila & 43 & 764 & 6.25 & 94734 & protein \\
\hline 313 & E4MWV7 & mRNA, clone: RTFL01-09-M07 & T. halophila & 2 & 106 & 7.42 & 10792 & protein \\
\hline 314 & E4MX84 & mRNA, clone: RTFL01-14-C02 & T. halophila & 2 & 30 & 5.54 & 55142 & protein \\
\hline 315 & E4MXV1 & mRNA, clone: RTFL01-35-G02 & T. halophila & 2 & 50 & 6.00 & 40626 & protein \\
\hline 316 & E5GBD5 & 60S ribosomal protein 19 & C. melo & 3 & 72 & 9.45 & 21994 & protein \\
\hline 317 & E5GBL8 & ATP-dependent clp protease & C. melo & 19 & 256 & 7.23 & 103416 & protein \\
\hline 318 & E5GBS0 & 40 S ribosomal protein $\mathrm{S} 24$ & C. melo & 3 & 85 & 10.64 & 15761 & protein \\
\hline 319 & E5LCX1 & C14 cysteine protease & S. demissum & 6 & 195 & 4.98 & 23974 & protein \\
\hline
\end{tabular}




\begin{tabular}{|c|c|c|c|c|c|c|c|c|}
\hline 320 & E5RDE1 & $26 \mathrm{~S}$ proteasome non-ATPase regulatory subunit & C. melo & 4 & 53 & 4.73 & 34256 & protein \\
\hline 321 & F2CQ90 & Predicted protein & H. vulgare & 2 & 52 & 7.12 & 14005 & protein \\
\hline 322 & F2CQY1 & Predicted protein & H. vulgare & 3 & 113 & 9.52 & 25529 & protein \\
\hline 323 & F2CRM6 & Predicted protein & H. vulgare & 4 & 97 & 5.52 & 121394 & protein \\
\hline 324 & F2CTR2 & Predicted protein & H. vulgare & 3 & 120 & 6.99 & 59167 & protein \\
\hline 325 & F2CWX1 & Predicted protein & H. vulgare & 25 & 299 & 6.10 & 86113 & protein \\
\hline 326 & F2CXV7 & Predicted protein & H. vulgare & 2 & 106 & 8.05 & 25809 & protein \\
\hline 327 & F2D1A7 & Predicted protein & H. vulgare & 35 & 254 & 9.80 & 17886 & protein \\
\hline 328 & F2D448 & Predicted protein & H. vulgare & 11 & 127 & 10.17 & 30607 & protein \\
\hline 329 & F2D690 & Predicted protein & H. vulgare & 2 & 156 & 4.37 & 12678 & protein \\
\hline 330 & F2D861 & Predicted protein & H. vulgare & 8 & 384 & 10.43 & 15765 & protein \\
\hline 331 & F2D8B8 & Proteasome subunit alpha type & H. vulgare & 9 & 124 & 7.43 & 27434 & protein \\
\hline 332 & F2D9E0 & Predicted protein & H. vulgare & 4 & 110 & 11.14 & 28037 & protein \\
\hline 333 & F2DAU4 & Predicted protein & H. vulgare & 35 & 398 & 7.56 & 62696 & protein \\
\hline 334 & F2DBR2 & Predicted protein & H. vulgare & 2 & 23 & 5.20 & 112909 & protein \\
\hline 335 & F2DBW7 & Predicted protein & H. vulgare & 5 & 31 & 9.55 & 12439 & protein \\
\hline 336 & F2DLC2 & Proteasome subunit alpha type & H. vulgare & 4 & 186 & 4.68 & 26087 & protein \\
\hline 337 & F2DUH3 & Predicted protein & H. vulgare & 4 & 53 & 5.63 & 60964 & protein \\
\hline 338 & F2E941 & Predicted protein & H. vulgare & 8 & 140 & 6.67 & 52201 & protein \\
\hline 339 & F2EE28 & Predicted protein & H. vulgare & 24 & 401 & 5.55 & 61280 & protein \\
\hline 340 & F2EG92 & Predicted protein & H. vulgar & 4 & 120 & 12.12 & 7602 & protein \\
\hline 341 & F2EKH0 & Predicted protein & H. vulgare & 9 & 258 & 4.60 & 31771 & protein \\
\hline 342 & F2EKQ2 & Predicted protein & H. vulgare & 9 & 58 & 10.33 & 28399 & protein \\
\hline 343 & F8WLB7 & $40 \mathrm{~S}$ ribosomal protein $\mathrm{S} 3$ & C. unshiu & 4 & 106 & 9.63 & 20599 & protein \\
\hline 344 & G2XK63 & Putative uncharacterized protein & Z. mays & 3 & 68 & 5.82 & 57701 & protein \\
\hline 345 & G3CHK8 & Eukaryotic translation initiation factor 3 subunit $\mathrm{E}$ & P. trifoliata & 3 & 41 & 6.14 & 51916 & protein \\
\hline 346 & H1ZY49 & Translation elongation factor 1-alpha & L. maackii & 20 & 96 & 5.00 & 11619 & protein \\
\hline 347 & H6VUT7 & Translation elongation factor & A. mongolicus & 2 & 111 & 4.63 & 25300 & protein \\
\hline 348 & I0J3D8 & Proteasome subunit B. type & A. halleri & 2 & 111 & 6.06 & 33367 & protein \\
\hline 349 & I6RZD4 & Putative NAD-dependent dehydrogenase 2 & E. coca & 2 & 71 & 5.83 & 27370 & protein \\
\hline 350 & J9QAL9 & Ribosomal protein L2 & A. corniculatum & 5 & 128 & 10.23 & 21703 & protein \\
\hline 351 & K3XEI1 & Uncharacterized protein & S. italica & 50 & 561 & 6.27 & 94876 & protein \\
\hline
\end{tabular}




\begin{tabular}{|c|c|c|c|c|c|c|c|c|}
\hline 352 & K3XGJ6 & Uncharacterized protein & S. italica & 11 & 103 & 5.22 & 58129 & protein \\
\hline 353 & K3XIP8 & Uncharacterized protein & S. italica & 4 & 50 & 6.40 & 43030 & protein \\
\hline 354 & K3XJZ1 & Uncharacterized protein & S. italica & 6 & 170 & 9.39 & 35932 & protein \\
\hline 355 & K3XKD1 & Uncharacterized protein & S. italica & 3 & 99 & 6.76 & 34429 & protein \\
\hline 356 & K3XVB0 & Uncharacterized protein & S. italica & 6 & 125 & 7.81 & 87653 & protein \\
\hline 357 & K3XX30 & Uncharacterized protein & S. italica & 7 & 93 & 5.07 & 47991 & protein \\
\hline 358 & $\mathrm{~K} 3 \mathrm{XX} 43$ & Uncharacterized protein & S. italica & 9 & 110 & 6.29 & 48119 & protein \\
\hline 359 & K3XZ10 & Uncharacterized protein & S. italica & 7 & 140 & 6.20 & 26286 & protein \\
\hline 360 & K3XZE3 & Uncharacterized protein & S. italica & 3 & 147 & 5.27 & 23153 & protein \\
\hline 361 & K3XZW6 & Uncharacterized protein & S. italica & 3 & 36 & 10.55 & 15740 & protein \\
\hline 362 & K3Y9X5 & Uncharacterized protein & S. italica & 13 & 364 & 10.39 & 24963 & protein \\
\hline 363 & K3Y9Y1 & Uncharacterized protein & S. italica & 5 & 41 & 10.07 & 24396 & protein \\
\hline 364 & K3YPS9 & Uncharacterized protein & S. italica & 13 & 134 & 5.24 & 97789 & protein \\
\hline 365 & K3YR06 & Uncharacterized protein & S. italica & 28 & 828 & 6.16 & 64225 & protein \\
\hline 366 & K3YR24 & Uncharacterized protein & S. italica & 11 & 226 & 7.91 & 62012 & protein \\
\hline 367 & K3YR95 & Uncharacterized protein & S. italica & 26 & 322 & 7.65 & 58776 & protein \\
\hline 368 & K3YVE3 & Uncharacterized protein & S. italica & 20 & 204 & 5.53 & 25864 & protein \\
\hline 369 & K3YZ28 & Uncharacterized protein & S. italica & 2 & 78 & 10.59 & 15971 & protein \\
\hline 370 & K3Z3M1 & Uncharacterized protein & S. italica & 14 & 247 & 7.06 & 102144 & protein \\
\hline 371 & K3ZH11 & Uncharacterized protein & S. italica & 12 & 61 & 5.38 & 118046 & protein \\
\hline 372 & K3ZH26 & Uncharacterized protein & S. italica & 10 & 54 & 6.07 & 120414 & protein \\
\hline 373 & K3ZU91 & Uncharacterized protein & S. italica & 6 & 71 & 8.70 & 42567 & protein \\
\hline 374 & K3ZVI3 & Uncharacterized protein & S. italica & 11 & 80 & 5.16 & 33393 & protein \\
\hline 375 & K3ZW60 & Uncharacterized protein & S. italica & 4 & 28 & 9.76 & 29913 & protein \\
\hline 376 & K3ZW85 & Uncharacterized protein & S. italica & 15 & 74 & 10.35 & 29312 & protein \\
\hline 377 & K3ZWX8 & Uncharacterized protein & S. italica & 14 & 56 & 11.49 & 24135 & protein \\
\hline 378 & K3ZX77 & Uncharacterized protein & S. italica & 10 & 73 & 11.34 & 21045 & protein \\
\hline 379 & K4A6E4 & Uncharacterized protein & S. italica & 5 & 31 & 5.16 & 83215 & protein \\
\hline 380 & K4A7N1 & Uncharacterized protein & S. italica & 13 & 166 & 6.02 & 61055 & protein \\
\hline 381 & K4A7Q9 & Uncharacterized protein & S. italica & 13 & 197 & 5.74 & 60770 & protein \\
\hline 382 & K4AAQ4 & Uncharacterized protein & S. italica & 6 & 128 & 10.56 & 44399 & protein \\
\hline 383 & $\mathrm{~K} 4 \mathrm{ACC} 4$ & Uncharacterized protein & S. italica & 8 & 64 & 5.66 & 38305 & protein \\
\hline
\end{tabular}




\begin{tabular}{|c|c|c|c|c|c|c|c|c|}
\hline 384 & $\mathrm{O} 23963$ & Elongation factor $\mathrm{Tu}$ & G. $\max$ & 21 & 190 & 5.14 & 38044 & protein \\
\hline 385 & O65879 & Translation initiation factor & P. sativum & 7 & 75 & 7.46 & 46184 & protein \\
\hline 386 & Q0W9E2 & B. chaperonin 60 & S. commersonii & 17 & 478 & 7.27 & 63333 & protein \\
\hline 387 & Q1KUM7 & Putative uncharacterized protein & C. spinosa & 5 & 110 & 6.62 & 59525 & protein \\
\hline 388 & Q39986 & Cysteine proteinase & Hemerocallis sp. & 4 & 155 & 5.50 & 40006 & protein \\
\hline 389 & Q40556 & Protein phosphatase $2 \mathrm{~A}$ & N. tabacum & 17 & 81 & 5.17 & 66079 & protein \\
\hline 390 & Q6B443 & $26 \mathrm{~S}$ proteasome B. subunit & L. minor & 11 & 248 & 9.72 & 12530 & protein \\
\hline 391 & Q6H8J2 & 40S ribosomal protein S9 & C. roseus & 13 & 65 & 10.20 & 23015 & protein \\
\hline 392 & Q6SKP4 & Ribosomal protein L3 & S. lycopersicum & 14 & 123 & 10.08 & 44836 & protein \\
\hline 393 & Q6Y1C4 & $40 \mathrm{~S}$ ribosomal protein $\mathrm{S} 9$ & L. saligna & 23 & 58 & 10.95 & 14138 & protein \\
\hline 394 & Q84QC6 & Ubiquitin-conjugating enzyme & H. vulgare & 7 & 43 & 7.02 & 16667 & protein \\
\hline 395 & Q8GZD8 & Leucine aminopeptidase 2 , chloroplastic & S. lycopersicum & 10 & 134 & 7.80 & 60813 & protein \\
\hline 396 & Q8L994 & T-complex protein 1 subunit delta & A. thaliana & 3 & 69 & 7.68 & 58177 & protein \\
\hline 397 & Q8LAW8 & Serine/threonine-protein phosphatase & A. thaliana & 4 & 141 & 4.96 & 35539 & protein \\
\hline 398 & Q8RVT3 & Elongation factor EF-2 & P. sativum & 35 & 340 & 6.18 & 55623 & protein \\
\hline 399 & Q948V5 & Mitochondrial processing peptidase alpha subunit & M. alba & 3 & 38 & 8.25 & 54978 & protein \\
\hline 400 & Q9ATF4 & Ribosomal protein L33 & C. sativa & 3 & 116 & 10.58 & 12831 & protein \\
\hline 401 & Q9FRW7 & Aspartic proteinase 3 & N. alata & 2 & 85 & 5.22 & 55666 & protein \\
\hline 402 & Q9SF16 & AT3g11830/F26K24_12 & A. thaliana & 9 & 92 & 6.39 & 60195 & protein \\
\hline 403 & Q9STA4 & Cysteine protease & M. sativa & 2 & 27 & 5.57 & 22875 & protein \\
\hline 404 & Q9SZ27 & Putative aspartate-tRNA ligase & A. thaliana & 2 & 41 & 6.37 & 57768 & protein \\
\hline 405 & Q9T2N3 & Chaperonin-60 alpha 1 fragment (Fragments) & B. napus & 2 & 45 & 4.01 & 6261 & protein \\
\hline 406 & Q9XEW9 & Elongation factor 1-alpha & L. longiflorum & 61 & 388 & 9.07 & 49711 & protein \\
\hline 407 & Q9ZVY6 & $\mathrm{T} 25 \mathrm{~N} 20.17$ & A. thaliana & 5 & 48 & 5.58 & 90732 & protein \\
\hline 408 & A0ZQA9 & ATP synthase subunit B. & P. baccatum & 31 & 334 & 5.40 & 52897 & photosynthesis \\
\hline 409 & A4UTS5 & Chloroplast ferredoxin-NADP+ reductase & P. sativum & 11 & 180 & 8.40 & 40392 & photosynthesis \\
\hline 410 & A8JHR9 & Glyceraldehyde 3-phosphate dehydrogenase, dominant splicing variant & C. reinhardtii & 6 & 157 & 8.95 & 39963 & photosynthesis \\
\hline 411 & A8MRE8 & Triosephosphate isomerase & A. thaliana & 15 & 185 & 7.49 & 32458 & photosynthesis \\
\hline 412 & B0M1B1 & Peroxisomal glycolate oxidase & G. $\max$ & 33 & 275 & 9.13 & 40825 & photosynthesis \\
\hline 413 & B3H4P2 & Glyceraldehyde-3-phosphate dehydrogenase (NADP+) (Phosphorylating) & A. thaliana & 6 & 64 & 7.77 & 37929 & photosynthesis \\
\hline 414 & B5LBN4 & Ribulose bisphosphate carboxylase large chain & B. alleghaniensis & 50 & 370 & 6.05 & 22478 & photosynthesis \\
\hline 415 & B6TI65 & Fructose-bisphosphate aldolase & Z. mays & 13 & 176 & 7.83 & 41924 & photosynthesis \\
\hline
\end{tabular}




\begin{tabular}{|c|c|c|c|c|c|c|c|c|}
\hline 416 & B6UDN1 & Fructose-bisphosphate aldolase & Z. mays & 9 & 173 & 7.96 & 41956 & photosynthesis \\
\hline 417 & C6T859 & B.-form rubisco activase & G. $\max$ & 21 & 571 & 7.24 & 48764 & photosynthesis \\
\hline 418 & D0F109 & Ribulose bisphosphate carboxylase large chain & S. dariensis & 36 & 269 & 7.68 & 19294 & photosynthesis \\
\hline 419 & D2CJB9 & ATP synthase subunit beta & B. multifida & 21 & 395 & 5.05 & 51275 & photosynthesis \\
\hline 420 & D2DMF5 & Chloroplast plastocyanin & N. benthamiana & 12 & 28 & 5.20 & 17202 & photosynthesis \\
\hline 421 & D6C638 & Ribulose-1,5-bisphosphate carboxylase/oxygenase large subunit & P. sp. PSN 1 & 91 & 904 & 6.58 & 50770 & photosynthesis \\
\hline 422 & D8L9Q5 & Fructose-bisphosphate aldolase & T. aestivum & 13 & 351 & 8.09 & 41564 & photosynthesis \\
\hline 423 & E1AXT8 & Glycolate oxidase & N. benthamiana & 19 & 128 & 9.00 & 40627 & photosynthesis \\
\hline 424 & E1ZKK0 & Putative uncharacterized protein & C. variabilis & 9 & 167 & 7.20 & 35948 & photosynthesis \\
\hline 425 & E4MWP5 & mRNA, clone: RTFL01-15-G22 & T. halophila & 20 & 58 & 6.16 & 35258 & photosynthesis \\
\hline 426 & E4MXI1 & mRNA, clone: RTFL01-39-B07 & T. halophila & 20 & 348 & 6.98 & 48063 & photosynthesis \\
\hline 427 & F2DTB2 & Triosephosphate isomerase & H. vulgare & 19 & 104 & 7.42 & 32679 & photosynthesis \\
\hline 428 & F2DTL1 & Fructose-bisphosphate aldolase & H. vulgare & 9 & 185 & 8.35 & 41916 & photosynthesis \\
\hline 429 & G5DVX2 & Phosphoglycerate kinase & S. latifolia & 54 & 964 & 6.58 & 51352 & photosynthesis \\
\hline 430 & G5DXP1 & Fructose-bisphosphate aldolase & S. latifolia & 14 & 410 & 6.16 & 42771 & photosynthesis \\
\hline 431 & G8HAA9 & PLP-dependent aminotransferase & P. somniferum & 5 & 67 & 6.57 & 53606 & photosynthesis \\
\hline 432 & H2BBB1 & ATP synthase subunit alpha, chloroplastic & P. diguetii & 14 & 316 & 5.74 & 55710 & photosynthesis \\
\hline 433 & H6T8A4 & ATP synthase subunit B. & M. poeppigii & 50 & 1017 & 6.33 & 52110 & photosynthesis \\
\hline 434 & H6VP90 & ATP synthase subunit B. & C. lobatus & 19 & 387 & 5.19 & 47813 & photosynthesis \\
\hline 435 & К3ХІT0 & Uncharacterized protein & S. italica & 16 & 445 & 8.78 & 41915 & photosynthesis \\
\hline 436 & K3XVH0 & Uncharacterized protein & S. italica & 35 & 313 & 6.62 & 80203 & photosynthesis \\
\hline 437 & K3Z5U9 & Uncharacterized protein & S. italica & 31 & 465 & 6.47 & 49826 & photosynthesis \\
\hline 438 & K3ZA95 & Uncharacterized protein & S. italica & 26 & 35 & 8.53 & 19471 & photosynthesis \\
\hline 439 & K3ZIK0 & Uncharacterized protein & S. italica & 17 & 474 & 6.61 & 47729 & photosynthesis \\
\hline 440 & K3ZTJ3 & Uncharacterized protein & S. italica & 14 & 269 & 8.94 & 47124 & photosynthesis \\
\hline 441 & K4A7I4 & Uncharacterized protein & S. italica & 19 & 477 & 5.40 & 61666 & photosynthesis \\
\hline 442 & O03843 & Ribulose-bisphosphate carboxylase & T. glandulifera & 150 & 1940 & 6.04 & 53346 & photosynthesis \\
\hline 443 & $\mathrm{O} 22143$ & At2g45290/F4L23.20 & A. thaliana & 14 & 218 & 5.96 & 69264 & photosynthesis \\
\hline 444 & Q1A5W2 & Ribulose-1,5-bisphosphate carboxylase/oxygenase large subunit & B. hatcheri & 69 & 542 & 6.43 & 47148 & photosynthesis \\
\hline 445 & Q2V3V9 & Putative peroxisomal (S)-2-hydroxy-acid oxidase 2 & A. thaliana & 14 & 302 & 8.80 & 40332 & photosynthesis \\
\hline 446 & Q38766 & Victorin binding protein & A. sativa & 5 & 57 & 6.96 & 112298 & photosynthesis \\
\hline 447 & Q39640 & Glycolate oxidase & C.. Kurokawa & 30 & 334 & 8.79 & 40345 & photosynthesis \\
\hline
\end{tabular}




\begin{tabular}{|c|c|c|c|c|c|c|c|c|}
\hline 448 & Q3LGW4 & Ribulose-1,5-bisphosphate carboxylase/oxygenase large subunit & F. benjamina & 10 & 181 & 4.67 & 9052 & photosynthesis \\
\hline 449 & Q58H58 & Chloroplast photosynthetic oxygen-evolving protein $33 \mathrm{kDa}$ subunit & N. benthamiana & 3 & 34 & 5.66 & 35374 & photosynthesis \\
\hline 450 & Q5GLI7 & Ribulose 1,5-bisphosphate carboxylase/oxygenase large subunit & E. ghellinckii & 153 & 1484 & 6.89 & 48283 & photosynthesis \\
\hline 451 & Q5KTY2 & Ribulose-1,5-bisphosphate carboxylase large subunit & A. sutepensis & 119 & 1409 & 6.92 & 51143 & photosynthesis \\
\hline 452 & Q7DM38 & Ribulose bisphosphate carboxylase small chain & P. vulgaris & 28 & 116 & 8.43 & 15877 & photosynthesis \\
\hline 453 & Q8L4S4 & Phosphoglycerate kinase & A. speltoides & 32 & 396 & 5.01 & 31433 & photosynthesis \\
\hline 454 & Q8MCW6 & Ribulose-1,5-bisphosphate carboxylase large subunit & M. racemosa & 160 & 1680 & 6.58 & 51631 & photosynthesis \\
\hline 455 & Q8MCX1 & Ribulose-1,5-bisphosphate carboxylase large subunit & C. axillaris & 120 & 1282 & 6.58 & 52386 & photosynthesis \\
\hline 456 & Q8MCY2 & Ribulose bisphosphate carboxylase large chain & V. baccifera & 36 & 346 & 6.64 & 48739 & photosynthesis \\
\hline 457 & Q8VWP3 & Glyceraldehyde-3-phosphate dehydrogenase & C. аппиит & 9 & 122 & 6.70 & 33465 & photosynthesis \\
\hline 458 & Q9GD57 & Ribulose 5-bisphosphate carboxylase, large subunit & P. yemenensis & 183 & 1688 & 6.89 & 51857 & photosynthesis \\
\hline 459 & Q9SXX4 & Fructose-bisphosphate aldolase & N. paniculata & 16 & 376 & 7.33 & 42832 & photosynthesis \\
\hline 460 & Q9SXX5 & Fructose-bisphosphate aldolase & N. paniculata & 9 & 307 & 6.79 & 43074 & photosynthesis \\
\hline 461 & Q9TKG9 & Ribulose 1,5-bisphosphate carboxylase large subunit & R. tomentosum & 136 & 1336 & 7.11 & 52772 & photosynthesis \\
\hline 462 & Q9TKH9 & Ribulose 1,5-bisphosphate carboxylase large subunit & H. albomarginata & 108 & 1548 & 6.37 & 51833 & photosynthesis \\
\hline 463 & A0EJL8 & GDP-D-mannose-3',5'-epimerase & M. glabra & 14 & 137 & 6.21 & 42927 & redox \\
\hline 464 & A1BLP6 & Thioredoxin & M. truncatula & 11 & 69 & 6.16 & 12904 & redox \\
\hline 465 & A5JPK7 & Monodehydroascorbate reductase & V. vinifera & 24 & 157 & 6.24 & 47478 & redox \\
\hline 466 & A9Z0Q0 & Catalase & P. ginseng & 20 & 388 & 7.18 & 57016 & redox \\
\hline 467 & B4FPK8 & Cytochrome b5 & Z. mays & 4 & 101 & 5.30 & 14958 & redox \\
\hline 468 & C0LQA1 & GDP-D-mannose-3',5'-epimerase & M. domestica & 13 & 114 & 6.70 & 42854 & redox \\
\hline 469 & C5X277 & Putative uncharacterized protein $\mathrm{Sb} 02 \mathrm{~g} 026300$ & S. bicolor & 3 & 97 & 5.71 & 47330 & redox \\
\hline 470 & E4MVQ7 & mRNA, clone: RTFL01-03-C18 & T. halophila & 7 & 125 & 6.60 & 46631 & redox \\
\hline 471 & F2CQX6 & Thioredoxin reductase & H. vulgare & 3 & 59 & 6.23 & 35031 & redox \\
\hline 472 & G0WP59 & Superoxide dismutase $[\mathrm{Cu}-\mathrm{Zn}]$ & W. somnifera & 10 & 224 & 5.64 & 15782 & redox \\
\hline 473 & K0I7G7 & Thioredoxin peroxidase & N. tabacum & 8 & 64 & 8.03 & 29972 & redox \\
\hline 474 & K3ZI40 & Uncharacterized protein & S. italica & 6 & 90 & 5.01 & 56750 & redox \\
\hline 475 & $\mathrm{O} 24511$ & Catalase & N. tabacum & 19 & 133 & 6.86 & 57225 & redox \\
\hline 476 & O65156 & Glutathione peroxidase & Z. aethiopica & 4 & 51 & 9.73 & 26916 & redox \\
\hline 477 & Q2I826 & Monodehydroascorbate reductase III & P. patens & 4 & 29 & 5.82 & 46597 & redox \\
\hline 478 & Q5PY86 & NADH:cytochrome b5 reductase & V. fordii & 2 & 49 & 7.15 & 31341 & redox \\
\hline 479 & Q66PF9 & Monodehydroascorbate reductase I & P. sativum & 11 & 96 & 6.11 & 47436 & redox \\
\hline
\end{tabular}




\begin{tabular}{|c|c|c|c|c|c|c|c|c|}
\hline 480 & Q6XXZ2 & Gamma-glutamylcysteine synthetase & L. japonicus & 9 & 184 & 6.67 & 56232 & redox \\
\hline 481 & Q6ZXH7 & Putative ascorbate peroxidase & P. canadensis & 12 & 153 & 4.97 & 22506 & redox \\
\hline 482 & Q7XTK8 & Catalase & P. persica & 39 & 658 & 7.43 & 57289 & redox \\
\hline 483 & Q7XTK9 & Catalase & P. persica & 41 & 662 & 7.25 & 57262 & redox \\
\hline 484 & Q93YG1 & Monodehydroascorbate reductase & M. crystalL. & 15 & 115 & 6.81 & 51944 & redox \\
\hline 485 & Q96350 & Catalase & B. napus & 13 & 152 & 7.81 & 57170 & redox \\
\hline 486 & Q9FE12 & Peroxiredoxin & P. vulgaris & 7 & 223 & 5.33 & 28776 & redox \\
\hline 487 & Q9FEM4 & Catalase & B. pendula & 25 & 219 & 5.48 & 17247 & redox \\
\hline 488 & Q9SAT7 & Superoxide dismutase $[\mathrm{Cu}-\mathrm{Zn}]$ & P. tremuloides & 6 & 77 & 5.82 & 15420 & redox \\
\hline 489 & A9P9A2 & Putative uncharacterized protein & P. trichocarpa & 14 & 163 & 9.36 & 46272 & RNA \\
\hline 490 & E5GBL0 & Short-chain dehydrogenase/reductase & C. melo & 9 & 78 & 6.89 & 108844 & RNA \\
\hline 491 & Q2LFC1 & AGO4-2 & N. benthamiana & 9 & 92 & 8.78 & 102331 & RNA \\
\hline 492 & Q40270 & RNA-binding protein & M. crystallinum & 2 & 46 & 4.79 & 32001 & RNA \\
\hline 493 & Q8L5C2 & $110 \mathrm{kDa} 4 \mathrm{SNc}-$ Tudor domain protein & P. sativum & 4 & 30 & 7.36 & 108600 & RNA \\
\hline 494 & A1 KXW2 & 1-deoxy-D-xylulose 5-phosphate reductoisomerase & H. brasiliensis & 22 & 154 & 6.00 & 51428 & secondary metabolism \\
\hline 495 & A7BG58 & 2-C-methyl-D-erythritol 2,4-cyclodiphosphate synthase & C. jambhiri & 3 & 38 & 8.78 & 25632 & secondary metabolism \\
\hline 496 & A9ZMZ5 & Acetyl-CoA C-acetyltransferase & H. brasiliensis & 29 & 789 & 6.68 & 42039 & secondary metabolism \\
\hline 497 & B1NYI4 & Phytoene desaturase & N. benthamiana & 5 & 65 & 6.74 & 65942 & secondary metabolism \\
\hline 498 & B2Z6P0 & 4-Coumarate:CoA ligase & P. trichocarpa & 3 & 63 & 5.69 & 59541 & secondary metabolism \\
\hline 499 & B4FYM0 & Acetyl-CoA acetyltransferase, cytosolic 2 & Z. mays & 7 & 205 & 7.66 & 43450 & secondary metabolism \\
\hline 500 & B5BLW2 & Carotenoid cleavage dioxygenase 1 & M. truncatula & 5 & 58 & 6.79 & 61063 & secondary metabolism \\
\hline 501 & B6UDL5 & Hydroxymethylbutenyl 4-diphosphate synthase & Z. mays & 29 & 412 & 5.97 & 82691 & secondary metabolism \\
\hline 502 & B7UCR9 & 1-deoxy-d-xylulose 5-phosphate reductoisomerase & S. miltiorrhiza & 12 & 152 & 6.40 & 51960 & secondary metabolism \\
\hline 503 & С0Р9J6 & Aminoaldehyde dehydrogenase 1 & Z. mays & 4 & 49 & 5.45 & 55733 & secondary metabolism \\
\hline 504 & C1IC54 & Cinnamyl alcohol dehydrogenase & G. hirsutum & 9 & 48 & 6.25 & 39574 & secondary metabolism \\
\hline 505 & C1M2W0 & Somatic embryogenesis cinnamyl alcohol dehydrogenase 1 & C. sativus & 11 & 203 & 7.44 & 39462 & secondary metabolism \\
\hline 506 & E2FYC3 & Caffeic acid O-methyltransferase & C. sinensis & 2 & 80 & 6.05 & 40149 & secondary metabolism \\
\hline 507 & E4MXV2 & mRNA, clone: RTFL01-41-F15 & T. halophila & 10 & 90 & 6.09 & 64768 & secondary metabolism \\
\hline 508 & G8HAB1 & PLP-dependent aminotransferase & P. somniferum & 4 & 78 & 7.09 & 50587 & secondary metabolism \\
\hline 509 & K3XIH5 & Uncharacterized protein & S. italica & 4 & 28 & 6.44 & 44308 & secondary metabolism \\
\hline 510 & K3YQC4 & Uncharacterized protein & S. italica & 7 & 49 & 6.33 & 78439 & secondary metabolism \\
\hline 511 & K4A9K9 & Uncharacterized protein & S. italica & 2 & 37 & 5.83 & 52168 & secondary metabolism \\
\hline
\end{tabular}




\begin{tabular}{|c|c|c|c|c|c|c|c|c|}
\hline 512 & Q3KN68 & Isoflavone reductase-like protein 5 & V. vinifera & 17 & 170 & 6.10 & 33865 & secondary metabolism \\
\hline 513 & Q8GZR6 & GcpE & S. lycopersicum & 22 & 205 & 6.11 & 82805 & secondary metabolism \\
\hline 514 & Q8L8H6 & Phytoene desaturase & T. erecta & 4 & 57 & 6.70 & 38216 & secondary metabolism \\
\hline 515 & A2V880 & G protein B.-subunit-like protein & N. tabacum & 5 & 91 & 7.09 & 16100 & signalling \\
\hline 516 & A6MIZ2 & Trehalose-phosphate synthase 5 & P. patens & 5 & 104 & 5.58 & 97638 & signalling \\
\hline 517 & A8IXU7 & Phototropin & C. reinhardtii & 12 & 74 & 8.47 & 81928 & signalling \\
\hline 518 & A8J195 & Small rab-related GTPase & C. reinhardtii & 5 & 124 & 7.46 & 23825 & signalling \\
\hline 519 & A9P8Q7 & Predicted protein & P. trichocarpa & 36 & 406 & 4.84 & 28824 & signalling \\
\hline 520 & B6TVN4 & Phospholipase C & Z. mays & 3 & 51 & 8.43 & 46868 & signalling \\
\hline 521 & F2CQ27 & Predicted protein & H. vulgare & 11 & 54 & 6.66 & 25460 & signalling \\
\hline 522 & F2CRF1 & Predicted protein & H. vulgare & 46 & 387 & 4.88 & 29361 & signalling \\
\hline 523 & F2CT86 & Predicted protein & H. vulgare & 7 & 140 & 7.47 & 22104 & signalling \\
\hline 524 & G5DW29 & Putative calreticulin & S. latifolia & 6 & 54 & 6.23 & 48820 & signalling \\
\hline 525 & I6YMA7 & Uncharacterized protein & L. usitatissimum & 7 & 34 & 6.39 & 89937 & signalling \\
\hline 526 & $\mathrm{O} 22402$ & GDP dissociation inhibitor & N. tabacum & 21 & 104 & 5.67 & 50127 & signalling \\
\hline 527 & O81976 & 14-3-3 Protein & G. $\max$ & 30 & 395 & 4.21 & 7619 & signalling \\
\hline 528 & Q39860 & GTPase & G. $\max$ & 7 & 47 & 5.34 & 24700 & signalling \\
\hline 529 & Q40463 & NTGB2 & N. tabacum & 7 & 153 & 6.19 & 15827 & signalling \\
\hline 530 & Q6H3X8 & $14-3-3$ protein isoform $16 R$ & S. tuberosum & 20 & 209 & 4.78 & 29032 & signalling \\
\hline 531 & Q706C9 & GDP dissociation inhibitor & M. truncatula & 5 & 84 & 5.88 & 50211 & signalling \\
\hline 532 & Q75ZE0 & 14-3-3 e-1 protein & N. tabacum & 44 & 490 & 4.78 & 29479 & signalling \\
\hline 533 & Q8LC80 & Putative calcium-binding protein, calreticulin & A. thaliana & 35 & 742 & 4.53 & 48385 & signalling \\
\hline 534 & Q9SCB1 & Rab11 GTPase & S. lycopersicum & 8 & 81 & 6.00 & 24289 & signalling \\
\hline 535 & Q9SXR9 & LeArcA1 protein & S. lycopersicum & 6 & 54 & 7.12 & 36363 & signalling \\
\hline 536 & A8I7T1 & Heat shock protein 90B & C. reinhardtii & 9 & 203 & 4.88 & 87547 & stress \\
\hline 537 & B4YYB3 & ST103-2 & T. halophila & 5 & 60 & 9.70 & 9286 & stress \\
\hline 538 & B6EBD6 & Heat shock protein $90-2$ & G. $\max$ & 43 & 448 & 5.07 & 80392 & stress \\
\hline 539 & B6SZ69 & Heat shock cognate $70 \mathrm{kDa}$ protein 2 & Z. mays & 43 & 712 & 5.19 & 71493 & stress \\
\hline 540 & B6U0V6 & Endoplasmin & Z. mays & 18 & 324 & 4.97 & 92831 & stress \\
\hline 541 & B6U237 & Heat shock $70 \mathrm{kDa}$ protein 4 & Z. mays & 8 & 109 & 5.43 & 94309 & stress \\
\hline 542 & B7SA66 & Hsp90-like protein & D. glomerata & 17 & 189 & 4.97 & 92918 & stress \\
\hline 543 & B9RZK1 & Major allergen Pru ar, putative & R. communis & 2 & 46 & 4.98 & 17294 & stress \\
\hline
\end{tabular}




\begin{tabular}{|c|c|c|c|c|c|c|c|c|}
\hline 544 & D0EJY9 & Molecular chaperone Hsp90-3 & N. benthamiana & 31 & 582 & 5.02 & 80638 & stress \\
\hline 545 & D2XNF3 & Chaperone protein DnaK & P. patens & 13 & 136 & 5.50 & 75674 & stress \\
\hline 546 & D7SY81 & Putative uncharacterized protein & V. vinifera & 4 & 88 & 5.48 & 12099 & stress \\
\hline 547 & E4MXI2 & mRNA, clone: RTFL01-39-D20 & T. halophila & 34 & 348 & 5.21 & 73890 & stress \\
\hline 548 & E4MXR5 & mRNA, clone: RTFL01-40-M04 & T. halophila & 24 & 373 & 5.90 & 73489 & stress \\
\hline 549 & F2D269 & Predicted protein & H. vulgare & 13 & 185 & 5.04 & 73306 & stress \\
\hline 550 & F2D884 & Predicted protein & H. vulgare & 98 & 1736 & 5.19 & 71476 & stress \\
\hline 551 & F2E2M4 & Predicted protein & H. vulgare & 13 & 244 & 5.29 & 92002 & stress \\
\hline 552 & $\mathrm{~F} 2 \mathrm{E} 4 \mathrm{C} 2$ & Predicted protein & H. vulgare & 60 & 838 & 5.25 & 72202 & stress \\
\hline 553 & K3Z4G6 & Uncharacterized protein & S. italica & 83 & 1517 & 5.25 & 71367 & stress \\
\hline 554 & K3ZA87 & Uncharacterized protein & S. italica & 5 & 42 & 8.87 & 18363 & stress \\
\hline 555 & K4A6U5 & Uncharacterized protein & S. italica & 55 & 920 & 5.34 & 71710 & stress \\
\hline 556 & O50036 & Heat shock 70 protein & S. oleracea & 41 & 393 & 5.30 & 76266 & stress \\
\hline 557 & Q08II7 & Heat shock protein 70 -like protein & L. sativa & 8 & 405 & 10.33 & 12539 & stress \\
\hline 558 & Q0MYQ7 & Germin-like protein 2 & V. vinifera & 3 & 157 & 8.41 & 22832 & stress \\
\hline 559 & Q4LDR0 & Heat shock protein & S. lycopersicum & 5 & 81 & 6.55 & 110564 & stress \\
\hline 560 & Q5EBY7 & Heat shock protein 70 & Z. mays & 46 & 680 & 4.78 & 41240 & stress \\
\hline 561 & Q8H6B6 & Chaperone protein HtpG & $X$. viscosa & 20 & 179 & 5.02 & 92957 & stress \\
\hline 562 & Q8LG68 & UDP-glucose 6-dehydrogenase & A. thaliana & 19 & 393 & 6.18 & 53591 & stress \\
\hline 563 & Q9ARB0 & Nbi-D protein & L. usitatissimum & 19 & 39 & 6.93 & 127688 & stress \\
\hline 564 & A7WPL0 & Putative reductase & N. tabacum & 13 & 142 & 8.09 & 44428 & TCA \\
\hline 565 & В3H477 & Fumarate hydratase 2 & A. thaliana & 2 & 128 & 6.76 & 45707 & TCA \\
\hline 566 & B5LAT6 & Dihydrolipoyl dehydrogenase & C. аппиит & 5 & 36 & 6.86 & 54133 & TCA \\
\hline 567 & B6SHD3 & Malate dehydrogenase & Z. mays & 62 & 767 & 8.32 & 42623 & TCA \\
\hline 568 & B6TCZ3 & Malate dehydrogenase & Z. mays & 47 & 676 & 7.74 & 41394 & TCA \\
\hline 569 & B6TJD7 & Isocitrate dehydrogenase subunit 1 & Z. mays & 4 & 55 & 7.14 & 40798 & TCA \\
\hline 570 & D3GQL1 & Aconitate hydratase 3 & C. clementina & 64 & 936 & 6.30 & 98669 & TCA \\
\hline 571 & D3GQL2 & Aconitate hydratase 2 & C. clementina & 80 & 828 & 6.54 & 98475 & TCA \\
\hline 572 & D9ZBV9 & Malate dehydrogenase & S. globosa & 13 & 265 & 4.92 & 13766 & TCA \\
\hline 573 & D9ZC79 & Malate dehydrogenase & S. globosa & 8 & 213 & 5.02 & 12369 & TCA \\
\hline 574 & E4MX73 & mRNA, clone: RTFL01-10-N01 & T. halophila & 12 & 399 & 5.83 & 39684 & TCA \\
\hline 575 & F2DAP7 & Malic enzyme & H. vulgare & 6 & 170 & 5.78 & 69436 & TCA \\
\hline
\end{tabular}




\begin{tabular}{|c|c|c|c|c|c|c|c|c|}
\hline 576 & F2DBE0 & Predicted protein & H. vulgare & 6 & 87 & 8.22 & 34595 & TCA \\
\hline 577 & F2DDZ5 & Predicted protein & H. vulgare & 2 & 71 & 5.48 & 40470 & TCA \\
\hline 578 & F2DGF3 & Predicted protein & H. vulgare & 7 & 94 & 6.81 & 115601 & TCA \\
\hline 579 & F2DIS4 & Malic enzyme & H. vulgare & 4 & 35 & 7.40 & 68411 & TCA \\
\hline 580 & F2E797 & Predicted protein & H. vulgare & 6 & 90 & 5.72 & 47111 & TCA \\
\hline 581 & F2EL27 & Predicted protein & H. vulgare & 9 & 98 & 6.57 & 68608 & TCA \\
\hline 582 & I1LCM5 & Uncharacterized protein & G. $\max$ & 4 & 65 & 6.96 & 47008 & TCA \\
\hline 583 & I6QZA8 & Malate dehydrogenase & E. coca & 100 & 1879 & 7.62 & 39915 & TCA \\
\hline 584 & K3XFR6 & Uncharacterized protein & S. italica & 27 & 337 & 7.46 & 66448 & TCA \\
\hline 585 & K3XJN7 & Uncharacterized protein & S. italica & 65 & 1004 & 7.85 & 35629 & TCA \\
\hline 586 & K3Y7B0 & Uncharacterized protein & S. italica & 17 & 60 & 8.70 & 48942 & TCA \\
\hline 587 & K3YG24 & Uncharacterized protein & S. italica & 58 & 561 & 7.31 & 107135 & TCA \\
\hline 588 & K3YRV8 & Uncharacterized protein & S. italica & 4 & 53 & 7.27 & 55802 & TCA \\
\hline 589 & K4A8Y9 & Uncharacterized protein & S. italica & 4 & 192 & 7.61 & 53531 & TCA \\
\hline 590 & K4ACE3 & Uncharacterized protein & S. italica & 93 & 1641 & 6.09 & 35803 & TCA \\
\hline 591 & $\mathrm{O} 24135$ & Citrate synthase & N. tabacum & 6 & 108 & 7.85 & 52744 & TCA \\
\hline 592 & O81609 & Nodule-enhanced malate dehydrogenase & P. sativum & 25 & 804 & 7.75 & 42106 & TCA \\
\hline 593 & P93133 & Isocitrate dehydrogenase [NADP] & E. globulus & 10 & 172 & 6.93 & 47106 & TCA \\
\hline 594 & Q42737 & NADP-malate dehydrogenase & F. trinervia & 2 & 79 & 6.46 & 46155 & TCA \\
\hline 595 & Q5ZFR7 & Malate dehydrogenase & P. major & 81 & 1429 & 6.54 & 36026 & TCA \\
\hline 596 & Q645N1 & Malate dehydrogenase & S. lycopersicum & 65 & 1409 & 8.72 & 36357 & TCA \\
\hline 597 & Q93WQ1 & Dihydrolipoyl dehydrogenase & B. gymnorhiza & 6 & 108 & 7.17 & 54389 & TCA \\
\hline 598 & Q9AXR6 & ATP:citrate lyase & C. аппиит & 26 & 360 & 7.39 & 66376 & TCA \\
\hline 599 & Q9M2T8 & 2-oxoglutarate dehydrogenase, E1 subunit-like protein & A. thaliana & 12 & 139 & 7.14 & 114888 & TCA \\
\hline 600 & Q9M6B3 & Malate dehydrogenase & V. vinifera & 133 & 3075 & 8.62 & 37137 & TCA \\
\hline 601 & Q9SW73 & Isocitrate dehydrogenase [NADP] & C. limon & 14 & 237 & 6.95 & 46823 & TCA \\
\hline 602 & Q9ZNX1 & NAD-dependent isocitrate dehydrogenase & N. tabacum & 2 & 90 & 7.52 & 40651 & TCA \\
\hline 603 & В6T0С0 & Heme-binding protein 2 & Z. mays & 2 & 142 & 4.78 & 23860 & tetrapyrrole synthesis \\
\hline 604 & B6TPE4 & Glutamate-1-semialdehyde 2,1-aminomutase & Z. mays & 16 & 163 & 6.55 & 50223 & tetrapyrrole synthesis \\
\hline 605 & E5GCQ7 & Glutamate-1-semialdehyde 2,1-aminomutase & C. melo & 6 & 158 & 6.61 & 50339 & tetrapyrrole synthesis \\
\hline 606 & A9CM22 & Voltage-dependent anion channel & N. tabacum & 5 & 101 & 8.32 & 29694 & transport \\
\hline 607 & B3VDR8 & Plasma membrane proton pump & C. sativus & 22 & 120 & 6.96 & 105581 & transport \\
\hline
\end{tabular}




\begin{tabular}{|c|c|c|c|c|c|c|c|c|}
\hline 608 & B6SVF4 & Oligopeptide transporter 9 & Z. mays & 6 & 44 & 6.47 & 87421 & transport \\
\hline 609 & E5GB77 & Adenine nucleotide translocator & C. melo & 5 & 104 & 9.77 & 42664 & transport \\
\hline 610 & F2CQQ3 & Predicted protein & H. vulgare & 32 & 909 & 5.16 & 54310 & transport \\
\hline 611 & F2CTZ5 & Predicted protein & H. vulgare & 29 & 803 & 5.23 & 68752 & transport \\
\hline 612 & I3NVX2 & V-type ATPase subunit A & S. europaea & 31 & 548 & 5.20 & 68845 & transport \\
\hline 613 & K3YQW5 & Uncharacterized protein & S. italica & 34 & 805 & 5.39 & 68637 & transport \\
\hline 614 & K3ZD99 & Uncharacterized protein & S. italica & 3 & 49 & 9.16 & 26325 & transport \\
\hline
\end{tabular}

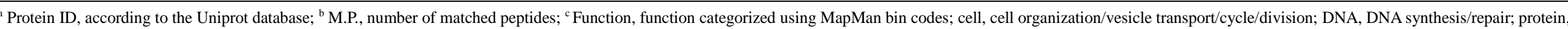

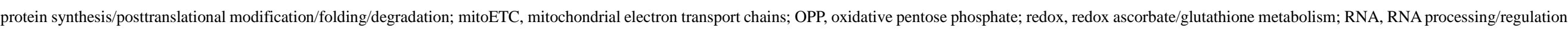
of transcription; TCA, tricarboxylic acid cycle; and misc, miscellaneous. 
Supplemental Table 12. Proteins Identified in L. japonica Immature Flower Buds using Polyethylene Glycol Fractionation.

\begin{tabular}{|c|c|c|c|c|c|c|c|c|}
\hline No. & Protein ID ${ }^{a}$ & Description & Species & M.P. ${ }^{b}$ & Score & $\mathrm{pI}$ & Mass (Da) & Function $^{\mathrm{c}}$ \\
\hline 1 & Q9SMC2 & Acetolactate synthase small subunit & N. plumbaginifolia & 5 & 102 & 7.27 & 50423 & amino acid metabolism \\
\hline 2 & B5LAW6 & Putative S-adenosylmethionine synthetase & C. аппиит & 14 & 229 & 6.05 & 43056 & amino acid metabolism \\
\hline 3 & B0FNB2 & Pyrroline-5-carboxylate synthetase & P. vulgaris & 4 & 47 & 6.62 & 78099 & amino acid metabolism \\
\hline 4 & B9DH71 & Ketol-acid reductoisomerase & A. thaliana & 8 & 272 & 6.99 & 64142 & amino acid metabolism \\
\hline 5 & B9SGJ6 & Ketol-acid reductoisomerase, chloroplast, putative & R. communis & 9 & 293 & 6.80 & 63712 & amino acid metabolism \\
\hline 6 & G8GJ68 & Adenosylhomocysteinase & L. usitatissimum & 34 & 587 & 5.99 & 61872 & amino acid metabolism \\
\hline 7 & Q5D6C3 & Adenosylhomocysteinase & A. thaliana & 51 & 772 & 6.10 & 53970 & amino acid metabolism \\
\hline 8 & K3YGV5 & Uncharacterized protein & S. italica & 4 & 73 & 8.10 & 62283 & amino acid metabolism \\
\hline 9 & A9NUN5 & Putative uncharacterized protein & P. sitchensis & 3 & 90 & 8.81 & 45137 & amino acid metabolism \\
\hline 10 & Q8LFK9 & 3-dehydroquinate synthase-like protein & A. thaliana & 4 & 118 & 7.15 & 48377 & amino acid metabolism \\
\hline 11 & A0FKE6 & Chloroplast threonine deaminase 1 & S. lycopersicum & 20 & 387 & 6.37 & 66653 & amino acid metabolism \\
\hline 12 & B2VQE0 & Methionine synthase & O. ramosa & 55 & 726 & 6.40 & 84862 & amino acid metabolism \\
\hline 13 & C8YNG6 & 3-ketoacyl CoA thiolase 1 & P. hybrida & 7 & 127 & 8.29 & 49405 & amino acid metabolism \\
\hline 14 & D2KZ81 & S-adenosylmethionine synthase & M. domestica & 7 & 333 & 4.94 & 24831 & amino acid metabolism \\
\hline 15 & K3ZSX0 & Uncharacterized protein & S. italica & 8 & 156 & 7.64 & 53564 & amino acid metabolism \\
\hline 16 & Q4H1G2 & Methionine synthase & B. vulgaris & 28 & 511 & 6.46 & 88546 & amino acid metabolism \\
\hline 17 & Q84RD8 & Adenosylhomocysteinase & M. truncatula & 43 & 629 & 6.01 & 53790 & amino acid metabolism \\
\hline 18 & Q8LGJ6 & Phosphoglycerate dehydrogenase-like protein & A. thaliana & 7 & 152 & 6.76 & 63556 & amino acid metabolism \\
\hline 19 & Q9S768 & Alanine aminotransferase & O. sativa & 2 & 195 & 6.65 & 53130 & amino acid metabolism \\
\hline 20 & Q9SRV5 & 5-methyltetrahydropteroyltriglutamate--homocysteine methyltransferase & A. thaliana & 34 & 477 & 6.51 & 84873 & amino acid metabolism \\
\hline 21 & B3H778 & Argininosuccinate synthase & A. thaliana & 4 & 52 & 7.72 & 49340 & amino acid metabolism \\
\hline 22 & B9H2U7 & Predicted protein & P. trichocarpa & 7 & 191 & 7.28 & 64204 & amino acid metabolism \\
\hline 23 & Q30DX9 & 2-isopropylmalate synthase & B. insularis & 2 & 99 & & 68109 & amino acid metabolism \\
\hline 24 & Q5GAQ3 & Putative alanine aminotransferase & Z. mays & 3 & 98 & 5.64 & 57003 & amino acid metabolism \\
\hline 25 & Q9SK84 & Class-II DAHP synthetase-like protein & A. thaliana & 11 & 114 & 8.32 & 58689 & amino acid metabolism \\
\hline 26 & A9PAK9 & Putative uncharacterized protein & P. trichocarpa & 6 & 54 & 6.89 & 50623 & amino acid metabolism \\
\hline 27 & A9RZ64 & Predicted protein & P. patens & 3 & 124 & 7.87 & 50746 & amino acid metabolism \\
\hline 28 & B9S7T6 & Transaminase mtnE, putative & R. communis & 6 & 55 & 7.28 & 50909 & amino acid metabolism \\
\hline 29 & D7LW57 & Ketol-acid reductoisomerase & A. lyrata & 10 & 408 & 6.80 & 64346 & amino acid metabolism \\
\hline 30 & G5DWR0 & Branched-chain-amino-acid aminotransferase & S. latifolia & 5 & 97 & 5.83 & 38581 & amino acid metabolism \\
\hline 31 & G9M8Q6 & S-adenosylmethionine synthase & L. grandiflorum & 41 & 409 & 8.53 & 36539 & amino acid metabolism \\
\hline
\end{tabular}




\begin{tabular}{|c|c|c|c|c|c|c|c|c|}
\hline 32 & K3YH65 & Uncharacterized protein & S. italica & 2 & 58 & 7.56 & 56313 & amino acid metabolism \\
\hline 33 & Q069K2 & 5-methyltetrahydropteroyltriglutamate--homocysteine methyltransferase & N. suaveolens & 66 & 786 & 6.62 & 85060 & amino acid metabolism \\
\hline 34 & Q4H1G1 & Adenosylhomocysteinase & B. vulgaris & 80 & 1413 & 6.19 & 54052 & amino acid metabolism \\
\hline 35 & F2CQP8 & Predicted protein & H. vulgare & 14 & 153 & 5.49 & 32811 & biodegradation of xenobiotics \\
\hline 36 & O04428 & Lactoylglutathione lyase & C. paradisi & 26 & 251 & 5.59 & 32737 & biodegradation of xenobiotics \\
\hline 37 & K3Z566 & Uncharacterized protein & S. italica & 10 & 177 & 7.77 & 59005 & C1-metabolism \\
\hline 38 & $\mathrm{O} 23254$ & Serine hydroxymethyltransferase & A. thaliana & 23 & 441 & 7.23 & 52141 & C1-metabolism \\
\hline 39 & B6TN41 & Regulator of ribonuclease activity $\mathrm{A}$ & Z. mays & 5 & 45 & 6.18 & 18390 & C1-metabolism \\
\hline 40 & K3XJA4 & Uncharacterized protein & S. italica & 2 & 101 & 8.07 & 39886 & C1-metabolism \\
\hline 41 & Q8LBY1 & Hydroxymethyltransferase & A. thaliana & 8 & 126 & 7.02 & 52129 & C1-metabolism \\
\hline 42 & F2DSZ0 & Predicted protein & H. vulgare & 32 & 617 & 5.14 & 50087 & cell \\
\hline 43 & Q0ZUN6 & Alpha-tubulin & C. scutata & 81 & 1802 & 6.19 & 44358 & cell \\
\hline 44 & K3XE63 & Uncharacterized protein & S. italica & 4 & 90 & 6.04 & 108302 & cell \\
\hline 45 & D6PPX2 & AT3G08530-like protein & N. paniculata & 7 & 150 & 4.56 & 20434 & cell \\
\hline 46 & E1ZQ18 & Putative uncharacterized protein & C. variabilis & 4 & 26 & 5.39 & 183606 & cell \\
\hline 47 & E1Z5R3 & Putative uncharacterized protein & C. variabilis & 47 & 1069 & 5.12 & 93194 & cell \\
\hline 48 & F2DR86 & Predicted protein & H. vulgare & 42 & 429 & 5.48 & 41914 & cell \\
\hline 49 & F2E3Y2 & Predicted protein & H. vulgare & 77 & 1694 & 5.16 & 65885 & cell \\
\hline 50 & Q9SQH4 & Actin bundling protein $\mathrm{ABP} 135$ & L. longiflorum & 5 & 38 & 5.97 & 107053 & cell \\
\hline 51 & F2DKP3 & Beta-adaptin-like protein & H. vulgare & 2 & 39 & 5.10 & 99927 & cell \\
\hline 52 & Q8H6N0 & Beta-tubulin 1 & G. hirsutum & 89 & 1476 & 4.87 & 50441 & cell \\
\hline 53 & A6YIK6 & Alpha-tubulin 4 & P. tremuloides & 46 & 733 & 5.01 & 50481 & cell \\
\hline 54 & F2YP47 & Beta-tubulin & Vanilla planifolia & 68 & 1217 & 4.87 & 50673 & cell \\
\hline 55 & K4A5J5 & Uncharacterized protein & S. italica & 10 & 259 & 5.08 & 100819 & cell \\
\hline 56 & O82564 & Actin 2 & A. phyllitidis & 56 & 706 & 5.49 & 41828 & cell \\
\hline 57 & A2Q5W0 & Alpha-tubulin & M. truncatula & 67 & 1809 & 5.10 & 50457 & cell \\
\hline 58 & A7KQH0 & B.-tubulin & E. grandis & 81 & 1267 & 4.94 & 50615 & cell \\
\hline 59 & B5M4B1 & Beta-tubulin & S. tuberosum & 95 & 1872 & 4.86 & 51166 & cell \\
\hline 60 & B6TJ90 & Histone H4 & Z. mays & 15 & 173 & 9.67 & 20120 & cell \\
\hline 61 & B6ZK00 & Peroxisomal biogenesis factor 11 family protein & G. $\max$ & 4 & 93 & 9.76 & 26114 & cell \\
\hline 62 & D0ELH5 & Peptidyl-prolyl cis-trans isomerase & C. sinensis & 3 & 102 & 8.46 & 18310 & cell \\
\hline 63 & D6PAY2 & Peptidyl-prolyl cis-trans isomerase & V. hybrid cultivar & 4 & 78 & 8.46 & 18582 & cell \\
\hline
\end{tabular}




\begin{tabular}{|c|c|c|c|c|c|c|c|c|}
\hline 64 & H9NIE1 & Cell division cycle protein 48 & C. sinensis & 79 & 1861 & 5.29 & 90644 & cell \\
\hline 65 & K3XE87 & Uncharacterized protein & S. italica & 7 & 139 & 5.90 & 106127 & cell \\
\hline 66 & K3YKV0 & Uncharacterized protein & S. italica & 18 & 403 & 5.54 & 87462 & cell \\
\hline 67 & K3Z679 & Uncharacterized protein & S. italica & 113 & 1832 & 4.88 & 50728 & cell \\
\hline 68 & K3Z913 & Uncharacterized protein & S. italica & 5 & 131 & 9.47 & 26793 & cell \\
\hline 69 & K4A4Z8 & Uncharacterized protein & S. italica & 14 & 193 & 6.96 & 137642 & cell \\
\hline 70 & K4A9V6 & Uncharacterized protein & S. italica & 58 & 1077 & 5.10 & 50519 & cell \\
\hline 71 & Q2TFP1 & Tubulin B4 & G. $\max$ & 118 & 2374 & 4.83 & 51083 & cell \\
\hline 72 & Q39834 & Clathrin heavy chain & G. $\max$ & 59 & 725 & 5.55 & 194430 & cell \\
\hline 73 & Q9XEN8 & Annexin & N. tabacum & 7 & 150 & 5.53 & 36029 & cell \\
\hline 74 & Q9ZQT0 & Actin & G. echinata & 30 & 707 & 6.19 & 17082 & cell \\
\hline 75 & E0D6S0 & Actin & G. bicolor & 23 & 395 & 5.31 & 41913 & cell \\
\hline 76 & K3Y4M8 & Uncharacterized protein & S. italica & 7 & 199 & 5.39 & 194893 & cell \\
\hline 77 & A5AW47 & Profilin & V. vinifera & 21 & 157 & 6.16 & 92852 & cell \\
\hline 78 & A5YRY0 & Alpha-tubulin 3 & P. tremuloides & 6 & 118 & 5.01 & 50353 & cell \\
\hline 79 & E3NYI6 & Peptidyl-prolyl cis-trans isomerase & A. diogoi & 17 & 97 & 8.44 & 18332 & cell \\
\hline 80 & F2CTA9 & Predicted protein & H. vulgare & 4 & 75 & 4.75 & 42321 & cell \\
\hline 81 & F2DDG9 & Predicted protein & H. vulgare & 6 & 100 & 5.66 & 52441 & cell \\
\hline 82 & F8RW92 & Peptidyl-prolyl cis-trans isomerase & H. brasiliensis & 8 & 171 & 8.44 & 18210 & cell \\
\hline 83 & F8WL60 & B. actin & R. stylosa & 59 & 902 & 5.27 & 33424 & cell \\
\hline 84 & G0XNX8 & Actin & H. brasiliensis & 111 & 1792 & 5.49 & 41897 & cell \\
\hline 85 & K3XZA2 & Uncharacterized protein & S. italica & 17 & 329 & 9.58 & 23221 & cell \\
\hline 86 & K3ZXR4 & Uncharacterized protein & S. italica & 4 & 118 & 7.24 & 16069 & cell \\
\hline 87 & Q06Z74 & Fibrillin 4 & C. canephora & 7 & 124 & 8.88 & 32064 & cell \\
\hline 88 & F2DJQ0 & Predicted protein & H. vulgare & 16 & 261 & 7.72 & 36458 & cell wall \\
\hline 89 & K3XI07 & Uncharacterized protein & S. italica & 11 & 247 & 8.73 & 47392 & cell wall \\
\hline 90 & Q6B6L9 & UDP-D-glucuronate decarboxylase & H. vulgare & 10 & 346 & 8.76 & 44245 & cell wall \\
\hline 91 & K4ABP3 & Uncharacterized protein & S. italica & 28 & 379 & 6.13 & 41769 & cell wall \\
\hline 92 & F6H740 & Putative uncharacterized protein & V. vinifera & 2 & 31 & 7.99 & 84234 & cell wall \\
\hline 93 & A5JVC6 & Putative uncharacterized protein & B. campestris & 9 & 64 & 6.25 & 34259 & cell wall \\
\hline 94 & B6T9G3 & Alpha-1,4-glucan-protein synthase 1 & Z. mays & 26 & 499 & 7.05 & 41278 & cell wall \\
\hline 95 & Q5ZF83 & Putative uncharacterized protein & P. major & 14 & 224 & 7.28 & 25390 & cell wall \\
\hline
\end{tabular}




\begin{tabular}{|c|c|c|c|c|c|c|c|c|}
\hline 96 & A5JVD4 & Putative uncharacterized protein & B. campestris & 8 & 54 & 6.99 & 30584 & cell wall \\
\hline 97 & K3ZUR5 & Uncharacterized protein & S. italica & 11 & 368 & 6.24 & 36749 & co-factor and vitamine metabolism \\
\hline 98 & Q2LFC3 & AGO1-2 & N. benthamiana & 8 & 201 & 9.31 & 109989 & development \\
\hline 99 & B4FL17 & Translationally-controlled tumor protein & Z. mays & 18 & 176 & 4.67 & 18773 & development \\
\hline 100 & F2DEC1 & Predicted protein & H. vulgare & 2 & 66 & 5.08 & 44443 & development \\
\hline 101 & K3XWC3 & Uncharacterized protein & S. italica & 3 & 171 & 9.00 & 58110 & development \\
\hline 102 & Q8LCW6 & Similar to late embryogenesis abundant proteins & A. thaliana & 6 & 57 & 4.87 & 36056 & development \\
\hline 103 & $\mathrm{O} 48903$ & Malate dehydrogenase & M. sativa & 16 & 171 & 8.29 & 38397 & gluconeogenesis \\
\hline 104 & Q645M9 & Glyoxisomal malate dehydrogenase & S. lycopersicum & 8 & 155 & 7.94 & 38007 & gluconeogenesis \\
\hline 105 & F2D319 & Citrate synthase & H. vulgare & 3 & 125 & 7.24 & 40252 & gluconeogenesis \\
\hline 106 & Q7XAP7 & Glyceraldehyde-3-phosphate dehydrogenase & H. cordata & 59 & 2207 & 8.34 & 24077 & glycolysis \\
\hline 107 & F2DY57 & Predicted protein & H. vulgare & 3 & 66 & 5.95 & 50880 & glycolysis \\
\hline 108 & K3YH95 & Uncharacterized protein & S. italica & 15 & 229 & 7.05 & 53771 & glycolysis \\
\hline 109 & $\mathrm{O} 23930$ & Phosphoenolpyruvate carboxylase & F. pringlei & 5 & 197 & 4.59 & 10308 & glycolysis \\
\hline 110 & K3XL78 & Triosephosphate isomerase & S. italica & 7 & 162 & 5.49 & 27278 & glycolysis \\
\hline 111 & B6SJZ8 & Glyceraldehyde-3-phosphate dehydrogenase, cytosolic & Z. mays & 57 & 2135 & 6.96 & 36614 & glycolysis \\
\hline 112 & В6Т3Р9 & Enolase & Z. mays & 8 & 291 & 5.82 & 48386 & glycolysis \\
\hline 113 & C4B8E5 & Glyceraldehyde-3-phosphate dehydrogenase & T. gesneriana & 94 & 3314 & 7.96 & 20964 & glycolysis \\
\hline 114 & D7NHW9 & 2-phospho-D-glycerate hydrolase & P. trifoliata & 18 & 453 & 5.78 & 47986 & glycolysis \\
\hline 115 & E4MY36 & mRNA, clone: RTFL01-37-B16 & T. halophila & 11 & 86 & 6.25 & 63555 & glycolysis \\
\hline 116 & E5GBV8 & Glyceraldehyde-3-phosphate dehydrogenase & C. melo & 62 & 2356 & 7.12 & 36754 & glycolysis \\
\hline 117 & F2D6I8 & Predicted protein & H. vulgare & 69 & 2643 & 7.14 & 36874 & glycolysis \\
\hline 118 & G9MA91 & Fructose-bisphosphate aldolase & L. grandiflorum & 5 & 202 & 6.28 & 37690 & glycolysis \\
\hline 119 & J3RTS9 & Glyceraldehyde-3-phosphate dehydrogenase & C. pentagona & 120 & 3365 & 7.23 & 32382 & glycolysis \\
\hline 120 & K3XWI6 & Uncharacterized protein & S. italica & 8 & 210 & 6.60 & 55489 & glycolysis \\
\hline 121 & K4A6V8 & Uncharacterized protein & S. italica & 7 & 149 & 7.03 & 70549 & glycolysis \\
\hline 122 & Q1I0X5 & Pyruvate kinase & C. аппиит & 8 & 40 & 6.84 & 56114 & glycolysis \\
\hline 123 & Q1WFH5 & Phosphoenolpyruvate carboxylase isoform 3 & C. hilariana & 17 & 139 & 8.94 & 41462 & glycolysis \\
\hline 124 & Q43833 & Glyceraldehyde 3-phosphate dehydrogenase & S. tuberosum & 51 & 1238 & 6.44 & 32598 & glycolysis \\
\hline 125 & Q6PKU2 & Putative glyceraldehyde-3-phosphate dehydrogenase & O. minor & 60 & 1849 & 7.09 & 22122 & glycolysis \\
\hline 126 & Q6T379 & Triosephosphate isomerase & S. chacoense & 4 & 31 & 5.99 & 27251 & glycolysis \\
\hline 127 & Q8H945 & Phosphoenolpyruvate carboxylase & L. japonicus & 43 & 527 & 5.88 & 111020 & glycolysis \\
\hline
\end{tabular}




\begin{tabular}{|c|c|c|c|c|c|c|c|c|}
\hline 128 & B3TLL4 & Triosephosphate isomerase & E. guineensis & 22 & 87 & 6.23 & 27476 & glycolysis \\
\hline 129 & B6TGL7 & Enolase & Z. mays & 10 & 368 & 6.23 & 50512 & glycolysis \\
\hline 130 & C0KZ35 & Glyceraldehyde-3-phosphate dehydrogenase & S. arboricola & 35 & 709 & 7.28 & 21225 & glycolysis \\
\hline 131 & K3XL57 & Uncharacterized protein & S. italica & 19 & 100 & 5.48 & 27435 & glycolysis \\
\hline 132 & K3XXC5 & Uncharacterized protein & S. italica & 43 & 319 & 7.06 & 42409 & glycolysis \\
\hline 133 & Q19TV8 & UDP-glucose pyrophosphorylase & C. melo & 28 & 319 & 7.17 & 52201 & glycolysis \\
\hline 134 & Q944T3 & Glyceraldehyde 3-phosphate dehydrogenase 2 & F. ananassa & 17 & 414 & 9.20 & 13954 & glycolysis \\
\hline 135 & Q4ZJ74 & Allene oxide synthase & H. brasiliensis & 2 & 100 & 8.65 & 58783 & hormone metabolism \\
\hline 136 & F2DLJ7 & Predicted protein & H. vulgare & 2 & 112 & 7.77 & 44423 & hormone metabolism \\
\hline 137 & F2DP49 & Predicted protein & H. vulgare & 5 & 128 & 8.63 & 25913 & hormone metabolism \\
\hline 138 & I1LJS6 & Lipoxygenase & G. $\max$ & 4 & 107 & 7.36 & 104140 & hormone metabolism \\
\hline 139 & P93698 & Lipoxygenase & V. unguiculata & 7 & 190 & 6.49 & 102731 & hormone metabolism \\
\hline 140 & B6TJT2 & Auxin-induced protein PCNT115 & Z. mays & 8 & 124 & 6.73 & 38553 & hormone metabolism \\
\hline 141 & D3G6B9 & Snakin-1 & S. bulbocastanum & 8 & 57 & 8.72 & 10442 & hormone metabolism \\
\hline 142 & K3Y823 & Uncharacterized protein & S. italica & 15 & 48 & 6.71 & 41387 & hormone metabolism \\
\hline 143 & $\mathrm{O} 24153$ & S-adenosyl-methionine-sterol-C-methyltransferase homolog & N. tabacum & 2 & 46 & 7.55 & 39707 & hormone metabolism \\
\hline 144 & Q4ZJ73 & 12-oxophytodienoate reductase & H. brasiliensis & 4 & 66 & 8.72 & 44571 & hormone metabolism \\
\hline 145 & Q5CCG4 & Acetyl-CoA carboxylase & A. myosuroides & 4 & 109 & 5.87 & 82215 & lipid metabolism \\
\hline 146 & B5LAS7 & Putative acetyl co-enzyme A carboxylase biotin carboxylase subunit & C. аппиит & 10 & 125 & 7.06 & 58977 & lipid metabolism \\
\hline 147 & B2KNE6 & Phospholipase D & H. annuиs & 10 & 189 & 5.52 & 92315 & lipid metabolism \\
\hline 148 & B5LAU5 & DH putative B.-hydroxyacyl-ACP dehydratase & C. аппиит & 9 & 48 & 9.29 & 24080 & lipid metabolism \\
\hline 149 & Q42793 & Acetyl CoA carboxylase & G. $\max$ & 18 & 182 & 6.32 & 253225 & lipid metabolism \\
\hline 150 & Q9XFX7 & Phospholipase D & C. plantagineum & 5 & 103 & 5.66 & 92228 & lipid metabolism \\
\hline 151 & F2DWX8 & Predicted protein & H. vulgare & 3 & 65 & 6.39 & 21692 & lipid metabolism \\
\hline 152 & F8WQS4 & Quinone reductase & N. benthamiana & 13 & 145 & 6.18 & 21229 & lipid metabolism \\
\hline 153 & G1DVA7 & Non-specific lipid-transfer protein & H. аппии & 4 & 43 & 8.41 & 12193 & lipid metabolism \\
\hline 154 & H9A1W2 & Acyl-activating enzyme 10 & C. sativa & 3 & 49 & 6.49 & 62013 & lipid metabolism \\
\hline 155 & K3ZT72 & Uncharacterized protein & S. italica & 5 & 88 & 9.48 & 47497 & lipid metabolism \\
\hline 156 & O81830 & Putative uncharacterized protein AT4g27270 & A. thaliana & 16 & 236 & 6.79 & 22355 & lipid metabolism \\
\hline 157 & Q08130 & Branching enzyme & M. esculenta & 4 & 28 & 4.70 & 17765 & major $\mathrm{CHO}$ metabolism \\
\hline 158 & A3QQY2 & Sucrose synthase & C. intybus & 11 & 92 & 6.39 & 92338 & major $\mathrm{CHO}$ metabolism \\
\hline 159 & C5X0Q9 & Sucrose synthase & S. bicolor & 7 & 44 & 6.86 & 92627 & major $\mathrm{CHO}$ metabolism \\
\hline
\end{tabular}




\begin{tabular}{|c|c|c|c|c|c|c|c|c|}
\hline 160 & В6ТВ29 & Fructokinase-2 & Z. mays & 18 & 61 & 5.58 & 35875 & major CHO metabolism \\
\hline 161 & F2E397 & Predicted protein & H. vulgare & 5 & 108 & 5.87 & 34933 & major CHO metabolism \\
\hline 162 & Q6VWJ5 & Fructokinase 3 & S. lycopersicum & 6 & 121 & 5.80 & 41803 & major CHO metabolism \\
\hline 163 & Q7XAE2 & Putative fructokinase 2 & P. integrifolia & 11 & 39 & 5.35 & 35181 & major $\mathrm{CHO}$ metabolism \\
\hline 164 & F2CRM1 & Predicted protein & H. vulgare & 36 & 410 & 5.83 & 43138 & metal handling \\
\hline 165 & B9ILW3 & Predicted protein & P. trichocarpa & 2 & 129 & 6.25 & 35485 & minor $\mathrm{CHO}$ metabolism \\
\hline 166 & F2D1V6 & Predicted protein & H. vulgare & 6 & 83 & 6.60 & 34598 & minor $\mathrm{CHO}$ metabolism \\
\hline 167 & G1JSJ5 & At1g59610 & A. thaliana & 9 & 202 & 9.04 & 100452 & misc \\
\hline 168 & K3ZS25 & Uncharacterized protein & S. italica & 5 & 145 & 6.39 & 61335 & misc \\
\hline 169 & K4A6N0 & Uncharacterized protein & S. italica & 10 & 173 & 7.78 & 75831 & misc \\
\hline 170 & A8MRF3 & $60 \mathrm{~S}$ ribosomal protein L18a-1 & A. thaliana & 3 & 44 & 9.73 & 36337 & misc \\
\hline 171 & B9HJ23 & Predicted protein & P. trichocarpa & 9 & 158 & 6.54 & 101700 & misc \\
\hline 172 & B3VMR3 & Epoxide hydrolase & N. benthamiana & 3 & 41 & 6.46 & 35842 & misc \\
\hline 173 & B6T6Q8 & Alcohol dehydrogenase class 3 & Z. mays & 8 & 232 & 7.02 & 41639 & misc \\
\hline 174 & B9GYJ8 & Predicted protein & P. trichocarpa & 2 & 59 & 4.67 & 37573 & misc \\
\hline 175 & E4MW95 & mRNA, clone: RTFL01-05-M24 & T. halophila & 3 & 74 & 5.45 & 79691 & misc \\
\hline 176 & E5GBJ6 & Alcohol dehydrogenase & C. melo & 3 & 76 & 8.47 & 40658 & misc \\
\hline 177 & K3Z7I7 & Uncharacterized protein & S. italica & 7 & 128 & 6.19 & 38341 & misc \\
\hline 178 & K4ADT5 & Uncharacterized protein & S. italica & 2 & 154 & 5.97 & 26876 & misc \\
\hline 179 & Q8L935 & B.-1,3-glucanase-like protein & A. thaliana & 3 & 38 & 9.39 & 48952 & misc \\
\hline 180 & Q9SA89 & FAD-binding and BBE domain-containing protein & A. thaliana & 5 & 76 & 8.60 & 60060 & misc \\
\hline 181 & C6GFP3 & ATP synthase subunit beta & G. hirsutum & 16 & 380 & 6.29 & 59987 & mitoETC \\
\hline 182 & G9FCM9 & ATP synthase subunit alpha & B. hygrometrica & 27 & 242 & 6.61 & 55364 & mitoETC \\
\hline 183 & G9FCP1 & NADH-quinone oxidoreductase subunit D & B. hygrometrica & 4 & 76 & 7.56 & 44776 & mitoETC \\
\hline 184 & Q5S817 & ATP synthase subunit alpha & H. madagascariensis & 33 & 553 & 6.3 & 46438 & mitoETC \\
\hline 185 & Q7YAM3 & NADH dehydrogenase subunit 7 & C. vulgaris & 2 & 89 & 6.98 & 45253 & mitoETC \\
\hline 186 & Q45Q23 & PHB2 & N. benthamiana & 7 & 170 & 9.50 & 31837 & mitoETC \\
\hline 187 & E5GBX7 & NADH-ubiquinone oxidoreductase flavoprotein & C. melo & 11 & 130 & 8.05 & 53578 & mitoETC \\
\hline 188 & K4A699 & Uncharacterized protein & S. italica & 9 & 437 & 6.16 & 81644 & mitoETC \\
\hline 189 & Q5MFD0 & ATPase subunit 8 & L. Bergthorsson & 3 & 60 & 8.84 & 15817 & mitoETC \\
\hline 190 & B5TM95 & NADH dehydrogenase subunit 7 & A. thaliana & 10 & 223 & 7.12 & 45555 & mitoETC \\
\hline 191 & Q34691 & ATP synthase subunit alpha & H. annuиs & 33 & 516 & 6.60 & 55766 & mitoETC \\
\hline
\end{tabular}




\begin{tabular}{|c|c|c|c|c|c|c|c|c|}
\hline 192 & Q6R9J5 & ATPase subunit 4 & Z. mays & 2 & 74 & 8.48 & 24941 & mitoETC \\
\hline 193 & Q9SAQ0 & ATP synthase subunit B. & N. sylvestris & 35 & 1110 & 6.06 & 59597 & mitoETC \\
\hline 194 & F2D5B1 & Predicted protein & H. vulgare & 2 & 77 & 9.00 & 10876 & mitoETC \\
\hline 195 & F2DJB5 & Predicted protein & H. vulgare & 17 & 224 & 9.32 & 12285 & mitoETC \\
\hline 196 & K3XL18 & Uncharacterized protein & S. italica & 6 & 90 & 6.28 & 28593 & mitoETC \\
\hline 197 & Q41534 & ATP synthase subunit B. & T. aestivum & 125 & 2262 & 5.86 & 59326 & mitoETC \\
\hline 198 & O04998 & Glutamine synthetase & M. truncatula & 5 & 59 & 5.73 & 39326 & N-metabolism \\
\hline 199 & B5LAU8 & Putative ferredoxin-dependent glutamate synthase 1 & C. аппиит & 13 & 182 & 6.54 & 179205 & N-metabolism \\
\hline 200 & Q40360 & NADH-dependent glutamate synthase & M. sativa & 12 & 81 & 6.27 & 242513 & $\mathrm{~N}$-metabolism \\
\hline 201 & B5LAU9 & Glutamine synthetase & C. аппиит & 15 & 169 & 6.93 & 47926 & $\mathrm{~N}$-metabolism \\
\hline 202 & F2E3I7 & Glutamine synthetase & H. vulgare & 12 & 127 & 5.48 & 39331 & N-metabolism \\
\hline 203 & E4MVJ5 & mRNA, clone: RTFL01-06-D09 & T. halophila & 3 & 50 & 8.91 & 40486 & not assigned \\
\hline 204 & Q9LY69 & Putative uncharacterized protein MAA21_90 & A. thaliana & 2 & 49 & 5.11 & 119971 & not assigned \\
\hline 205 & K3YSH1 & Uncharacterized protein & S. italica & 8 & 52 & 9.01 & 48860 & not assigned \\
\hline 206 & B4G1D2 & CBS domain protein & Z. mays & 4 & 56 & 9.33 & 22541 & not assigned \\
\hline 207 & Q5GI04 & Hypersensitive-induced reaction protein & C. аппиит & 4 & 89 & 6.42 & 31614 & not assigned \\
\hline 208 & F6H3T7 & Putative uncharacterized protein & V. vinifera & 15 & 174 & 6.11 & 40651 & not assigned \\
\hline 209 & Q9C6U3 & Putative uncharacterized protein $\mathrm{T} 8 \mathrm{G} 24.2$ & A. thaliana & 5 & 135 & 7.59 & 34907 & not assigned \\
\hline 210 & K3ZQ66 & Uncharacterized protein & S. italica & 9 & 51 & 4.92 & 124515 & not assigned \\
\hline 211 & Q8LCK7 & Putative uncharacterized protein & A. thaliana & 5 & 82 & 7.56 & 48349 & not assigned \\
\hline 212 & B4FH62 & NAD-dependent epimerase/dehydratase & Z. mays & 16 & 279 & 9.04 & 31917 & not assigned \\
\hline 213 & B6SVI5 & Putative uncharacterized protein & Z. mays & 10 & 34 & 8.67 & 24337 & not assigned \\
\hline 214 & Q6E438 & ACT11D09.4 & C. melo & 5 & 106 & 7.93 & 100881 & not assigned \\
\hline 215 & Q8L6J9 & Putative carbamoyl phosphate synthase large subunit & N. tabacum & 7 & 98 & 5.91 & 133241 & nucleotide metabolism \\
\hline 216 & E5GCQ8 & Inorganic pyrophosphatase & C. melo & 7 & 145 & 5.78 & 33109 & nucleotide metabolism \\
\hline 217 & K4A938 & Uncharacterized protein & S. italica & 2 & 41 & 6.79 & 53547 & nucleotide metabolism \\
\hline 218 & F2Z9R3 & Glucose-6-phosphate 1-dehydrogenase & N. benthamiana & 9 & 159 & 8.24 & 67846 & OPP \\
\hline 219 & D6PPS8 & AT3G02360-like protein & C. grandiflora & 7 & 275 & 9.22 & 21010 & OPP \\
\hline 220 & F2Z9R1 & Glucose-6-phosphate 1-dehydrogenase & N. benthamiana & 18 & 141 & 6.49 & 58894 & OPP \\
\hline 221 & K3XH89 & Uncharacterized protein & S. italica & 19 & 310 & 5.87 & 52978 & OPP \\
\hline 222 & Q1KUX5 & 6-phosphogluconate dehydrogenase, decarboxylating & C. spinosa & 25 & 435 & 6.06 & 53777 & OPP \\
\hline 223 & Q7XJH9 & Transaldolase & S. lycopersicum & 10 & 248 & 5.96 & 48062 & OPP \\
\hline
\end{tabular}




\begin{tabular}{|c|c|c|c|c|c|c|c|c|}
\hline 224 & Q9SH69 & 6-phosphogluconate dehydrogenase, decarboxylating & A. thaliana & 14 & 474 & 5.45 & 53686 & OPP \\
\hline 225 & $\mathrm{O} 22143$ & Transketolase-2, chloroplastic & A. thaliana & 8 & 136 & 5.96 & 69264 & OPP \\
\hline 226 & Q8LG34 & 6-phosphogluconate dehydrogenase, decarboxylating & A. thaliana & 6 & 226 & 5.80 & 53555 & OPP \\
\hline 227 & Q5NKW4 & Photosystem I reaction center subunit II, $20 \mathrm{kDa}$ & C. reinhardtii & 4 & 55 & 10.30 & 21442 & photosynthesis \\
\hline 228 & Q93XK4 & Chlorophyll a/b binding protein & P. contorta & 8 & 113 & 5.59 & 29128 & photosynthesis \\
\hline 229 & D4N5G0 & Alpha-form rubisco activase & G. $\max$ & 35 & 946 & 6.25 & 52531 & photosynthesis \\
\hline 230 & B6T416 & Ribulose bisphosphate carboxylase/oxygenase activase & Z. mays & 28 & 985 & 6.73 & 48079 & photosynthesis \\
\hline 231 & F2CXX0 & Predicted protein & H. vulgare & 2 & 73 & 5.50 & 30839 & photosynthesis \\
\hline 232 & E4MVN2 & mRNA, clone: RTFL01-06-F21 & T. halophila & 4 & 83 & 6.42 & 31122 & photosynthesis \\
\hline 233 & G9FCD2 & ATP synthase subunit alpha, chloroplastic & B. hygrometrica & 19 & 319 & 5.34 & 55392 & photosynthesis \\
\hline 234 & Q4FGD9 & ATP synthase epsilon chain, chloroplastic & G. biloba & 2 & 106 & 6.11 & 14962 & photosynthesis \\
\hline 235 & Q32121 & Ribulose bisphosphate carboxylase large chain & D. californica & 74 & 1208 & 8.18 & 40464 & photosynthesis \\
\hline 236 & Q8MCW3 & Ribulose bisphosphate carboxylase large chain & L. coelhoi & 65 & 1114 & 8.88 & 34008 & photosynthesis \\
\hline 237 & A0ZQA9 & ATP synthase subunit B. & P. baccatum & 18 & 255 & 5.40 & 52897 & photosynthesis \\
\hline 238 & A8MRE8 & Triosephosphate isomerase & A. thaliana & 5 & 119 & 7.49 & 32458 & photosynthesis \\
\hline 239 & B0M1B1 & Peroxisomal glycolate oxidase & G. $\max$ & 11 & 68 & 9.13 & 40825 & photosynthesis \\
\hline 240 & B4F8L7 & Glyceraldehyde-3-phosphate dehydrogenase B & Z. mays & 22 & 173 & 6.39 & 47663 & photosynthesis \\
\hline 241 & B6TI65 & Fructose-bisphosphate aldolase & Z. mays & 7 & 345 & 7.83 & 41924 & photosynthesis \\
\hline 242 & B6UDN1 & Fructose-bisphosphate aldolase & Z. mays & 6 & 189 & 7.96 & 41956 & photosynthesis \\
\hline 243 & E4MXI1 & mRNA, clone: RTFL01-39-B07 & T. halophila & 19 & 295 & 6.98 & 48063 & photosynthesis \\
\hline 244 & G5DVX2 & Phosphoglycerate kinase & S. latifolia & 25 & 506 & 6.58 & 51352 & photosynthesis \\
\hline 245 & G5DXP1 & Fructose-bisphosphate aldolase & S. latifolia & 7 & 291 & 6.16 & 42771 & photosynthesis \\
\hline 246 & H6VP90 & ATP synthase subunit B. & C. lobatus & 14 & 263 & 5.19 & 47813 & photosynthesis \\
\hline 247 & K3XVH0 & Uncharacterized protein & S. italica & 9 & 69 & 6.62 & 80203 & photosynthesis \\
\hline 248 & K3Z5U9 & Uncharacterized protein & S. italica & 19 & 200 & 6.47 & 49826 & photosynthesis \\
\hline 249 & K3ZTJ3 & Uncharacterized protein & S. italica & 7 & 90 & 8.94 & 47124 & photosynthesis \\
\hline 250 & K4A7I4 & Uncharacterized protein & S. italica & 15 & 393 & 5.40 & 61666 & photosynthesis \\
\hline 251 & $\mathrm{O} 03843$ & Ribulose-bisphosphate carboxylase & T. glandulifera & 114 & 1828 & 6.04 & 53346 & photosynthesis \\
\hline 252 & $\mathrm{O} 78327$ & Transketolase 1 & C. аппиит & 4 & 89 & 6.62 & 80398 & photosynthesis \\
\hline 253 & Q38766 & Victorin binding protein & A. sativa & 7 & 69 & 6.96 & 112298 & photosynthesis \\
\hline 254 & Q5KTY2 & Ribulose-1,5-bisphosphate carboxylase large subunit & A. sutepensis & 83 & 1025 & 6.92 & 51143 & photosynthesis \\
\hline 255 & Q8L4S4 & Phosphoglycerate kinase & A. speltoides & 22 & 261 & 5.01 & 31433 & photosynthesis \\
\hline
\end{tabular}




\begin{tabular}{|c|c|c|c|c|c|c|c|c|}
\hline 256 & Q8VWP3 & Glyceraldehyde-3-phosphate dehydrogenase & C. аппиит & 17 & 184 & 6.70 & 33465 & photosynthesis \\
\hline 257 & B8PRL9 & Ribulose bisphosphate carboxylase large chain & A. canadensis & 39 & 278 & 5.62 & 52414 & photosynthesis \\
\hline 258 & C3S8R0 & Ribulose-1,5-bisphosphate carboxylase/oxygenase large subunit & L. gracilis & 41 & 259 & 6.92 & 47818 & photosynthesis \\
\hline 259 & C6T859 & Beta-form rubisco activase & G. $\max$ & 9 & 216 & 7.24 & 48764 & photosynthesis \\
\hline 260 & K3ZA95 & Ribulose bisphosphate carboxylase small chain & S. italica & 9 & 59 & 8.53 & 19471 & photosynthesis \\
\hline 261 & Q8MCW6 & Ribulose-1,5-bisphosphate carboxylase large subunit & M. racemosa & 43 & 349 & 6.58 & 51631 & photosynthesis \\
\hline 262 & Q8MCY5 & Ribulose bisphosphate carboxylase large chain & T. cymosum & 28 & 210 & 7.56 & 44221 & photosynthesis \\
\hline 263 & Q9SC26 & ATP synthase subunit beta & S. californica & 10 & 229 & 5.24 & 53636 & photosynthesis \\
\hline 264 & Q9SUU0 & Serine hydroxymethyltransferase & A. thaliana & 4 & 161 & 8.05 & 50988 & photosynthesis \\
\hline 265 & A8JIB7 & Chaperonin $60 \mathrm{~A}$ & C. reinhardtii & 3 & 87 & 5.62 & 61911 & photosynthesis \\
\hline 266 & B6SHL1 & Photosystem I reaction center subunit XI & Z. mays & 2 & 41 & 9.95 & 23193 & photosynthesis \\
\hline 267 & D8L9Q5 & Fructose-bisphosphate aldolase & T. aestivum & 12 & 299 & 8.09 & 41564 & photosynthesis \\
\hline 268 & E4MWP5 & mRNA, clone: RTFL01-15-G22 & T. halophila & 17 & 116 & 6.16 & 35258 & photosynthesis \\
\hline 269 & G1FB60 & Cytochrome b559 subunit alpha & S. polyrrhiza & 7 & 137 & 4.83 & 9408 & photosynthesis \\
\hline 270 & K3XIT0 & Uncharacterized protein & S. italica & 19 & 375 & 8.78 & 41915 & photosynthesis \\
\hline 271 & K3XL01 & Uncharacterized protein & S. italica & 4 & 54 & 9.09 & 27473 & photosynthesis \\
\hline 272 & K3ZVK5 & Uncharacterized protein & S. italica & 19 & 33 & 6.57 & 32436 & photosynthesis \\
\hline 273 & Q43874 & Ribulose bisphosphate carboxylase small chain & P. vulgaris & 17 & 167 & 9.03 & 20355 & photosynthesis \\
\hline 274 & Q58H58 & Chloroplast photosynthetic oxygen-evolving protein $33 \mathrm{kDa}$ subunit & N. benthamiana & 20 & 160 & 5.66 & 35374 & photosynthesis \\
\hline 275 & Q5S7Y5 & Triosephosphate isomerase & C. reinhardtii & 18 & 87 & 7.65 & 30433 & photosynthesis \\
\hline 276 & Q2V4Q4 & $50 \mathrm{~S}$ ribosomal protein $\mathrm{L} 4$ & A. thaliana & 3 & 77 & 9.26 & 30124 & protein \\
\hline 277 & B3TLQ8 & Ribosomal protein & E. guineensis & 11 & 327 & 9.79 & 24908 & protein \\
\hline 278 & K3ZXL7 & Eukaryotic translation initiation factor $5 \mathrm{~A}$ & S. italica & 4 & 115 & 5.99 & 17613 & protein \\
\hline 279 & K3XVN4 & Uncharacterized protein & S. italica & 5 & 113 & 5.72 & 72670 & protein \\
\hline 280 & Q9FVU8 & Putative UDP-glucose:glycoprotein glucosyltransferase; 101200-91134 & A. thaliana & 7 & 104 & 5.77 & 189722 & protein \\
\hline 281 & F2E0C0 & Predicted protein & H. vulgare & 13 & 49 & 10.04 & 28326 & protein \\
\hline 282 & F2DQ10 & Predicted protein & H. vulgare & 16 & 279 & 5.19 & 98573 & protein \\
\hline 283 & Q41444 & Mitochondrial processing peptidase & S. tuberosum & 5 & 110 & 6.64 & 59463 & protein \\
\hline 284 & Q41445 & Mitochondrial processing peptidase & S. tuberosum & 2 & 37 & 6.73 & 60107 & protein \\
\hline 285 & Q8LFN3 & Putative chaperonin & A. thaliana & 21 & 165 & 6.21 & 59466 & protein \\
\hline 286 & K3Z3P5 & Uncharacterized protein & S. italica & 11 & 98 & 6.25 & 95578 & protein \\
\hline 287 & Q9SR15 & Putative tryptophanyl-tRNA synthetase & A. thaliana & 3 & 108 & 5.95 & 46010 & protein \\
\hline
\end{tabular}




\begin{tabular}{|c|c|c|c|c|c|c|c|c|}
\hline 288 & Q9AYS9 & Tat binding protein like protein & B. campestris & 30 & 724 & 5.03 & 47733 & protein \\
\hline 289 & K3XW85 & T-complex protein 1 subunit alpha & S. italica & 12 & 359 & 6.09 & 59355 & protein \\
\hline 290 & Q0ZII1 & Joka20 & N. plumbaginifolia & 2 & 92 & 8.94 & 17109 & protein \\
\hline 291 & B4FD78 & $60 \mathrm{~S}$ acidic ribosomal protein $\mathrm{P} 2 \mathrm{~B}$ & Z. mays & 11 & 300 & 4.27 & 11693 & protein \\
\hline 292 & A8MS83 & $60 \mathrm{~S}$ ribosomal protein L23a-2 & A. thaliana & 13 & 127 & 10.17 & 16733 & protein \\
\hline 293 & B4FP40 & ADP-ribosylation factor & Z. mays & 9 & 204 & 6.95 & 20710 & protein \\
\hline 294 & B4FUS2 & 40S ribosomal protein $\mathrm{S} 18$ & Z. mays & 7 & 48 & 10.74 & 17741 & protein \\
\hline 295 & A8MQP6 & Nascent polypeptide-associated complex subunit alpha-like protein 4 & A. thaliana & 4 & 112 & 4.42 & 23100 & protein \\
\hline 296 & F2DN74 & Methionine--tRNA ligase & H. vulgare & 2 & 64 & 7.01 & 72371 & protein \\
\hline 297 & K3ZTA6 & Uncharacterized protein & S. italica & 26 & 473 & 6.21 & 49589 & protein \\
\hline 298 & Q9M084 & AT4G31180 protein & A. thaliana & 3 & 148 & 6.23 & 63391 & protein \\
\hline 299 & Q9FY49 & Leukotriene-A4 hydrolase & A. thaliana & 4 & 67 & 5.24 & 69621 & protein \\
\hline 300 & B3TM44 & Ribosomal protein S7 & E. guineensis & 2 & 68 & 9.77 & 21923 & protein \\
\hline 301 & Q940P8 & T-complex protein 1 subunit beta & A. thaliana & 19 & 336 & 5.87 & 57763 & protein \\
\hline 302 & B4FLQ8 & $26 \mathrm{~S}$ proteasome non-ATPase regulatory subunit 14 & Z. mays & 7 & 93 & 6.76 & 34443 & protein \\
\hline 303 & Q7XJB0 & Eukaryotic translation initiation factor iso $4 \mathrm{E}$ & L. sativa & 2 & 154 & 5.69 & 22125 & protein \\
\hline 304 & E1ZP98 & Putative uncharacterized protein & C. variabilis & 18 & 305 & 5.74 & 93462 & protein \\
\hline 305 & K3Y516 & Uncharacterized protein & S. italica & 41 & 803 & 6.61 & 102308 & protein \\
\hline 306 & B1PBX8 & Putative uncharacterized protein & A. lyrata & 11 & 243 & 8.94 & 19793 & protein \\
\hline 307 & B6TDT1 & $26 \mathrm{~S}$ protease regulatory subunit $6 \mathrm{~B}$ & Z. mays & 18 & 305 & 6.07 & 46827 & protein \\
\hline 308 & А9РВT8 & Putative uncharacterized protein & P. trichocarpa & 4 & 78 & 6.67 & 48985 & protein \\
\hline 309 & $\mathrm{H} 2 \mathrm{BC} 31$ & 50S ribosomal protein L14, chloroplastic & B. liliputana & 2 & 160 & 10.32 & 13665 & protein \\
\hline 310 & F2DRC5 & Predicted protein & H. vulgare & 20 & 517 & 5.92 & 57700 & protein \\
\hline 311 & G5DXC9 & $26 \mathrm{~S}$ proteasome regulatory subunit $\mathrm{N} 1$ & S. latifolia & 19 & 502 & 5.16 & 97731 & protein \\
\hline 312 & Q94KB0 & Importin subunit alpha & C. аппиит & 9 & 259 & 5.39 & 59910 & protein \\
\hline 313 & A1YUL9 & Importin subunit alpha & $N$. benthamiana & 17 & 363 & 5.38 & 59114 & protein \\
\hline 314 & A8MRH4 & $60 \mathrm{~S}$ ribosomal protein $\mathrm{L} 7-2$ & A. thaliana & 4 & 40 & 10.10 & 21540 & protein \\
\hline 315 & A9PD17 & Predicted protein & P. trichocarpa & 35 & 615 & 5.82 & 47103 & protein \\
\hline 316 & A9PE52 & Gamma class glutathione transferase EF1Bgamma2 & P. trichocarpa & 10 & 212 & 5.80 & 48127 & protein \\
\hline 317 & B3TLL5 & 40S ribosomal protein S19 & E. guineensis & 12 & 44 & 10.04 & 16202 & protein \\
\hline 318 & B3TLN9 & Cytoplasmic ribosomal protein S15a & E. guineensis & 4 & 46 & 9.89 & 14862 & protein \\
\hline 319 & B3TLP3 & Translation elongation factor EF-1 B. chain & E. guineensis & 10 & 216 & 4.69 & 24523 & protein \\
\hline
\end{tabular}




\begin{tabular}{|c|c|c|c|c|c|c|c|c|}
\hline 320 & B3TLP4 & Ribosomal protein L30 & E. guineensis & 5 & 162 & 9.72 & 12451 & protein \\
\hline 321 & B3TLY3 & Ribosomal protein L15 & E. guineensis & 2 & 127 & 11.49 & 24371 & protein \\
\hline 322 & В3TM23 & $40 \mathrm{~S}$ ribosomal protein $\mathrm{S} 4$ & E. guineensis & 15 & 148 & 10.24 & 30023 & protein \\
\hline 323 & В3TM36 & $40 \mathrm{~S}$ ribosomal protein $\mathrm{S} 8$ & E. guineensis & 3 & 175 & 10.43 & 25347 & protein \\
\hline 324 & В3TM39 & Ribosomal protein S14 & E. guineensis & 11 & 232 & 10.70 & 16428 & protein \\
\hline 325 & B6TGB2 & Elongation factor 1-gamma 3 & Z. mays & 12 & 288 & 6.37 & 47357 & protein \\
\hline 326 & B6THG9 & $60 \mathrm{~S}$ ribosomal protein $\mathrm{L} 5-1$ & Z. mays & 13 & 338 & 9.32 & 34462 & protein \\
\hline 327 & B6TLT1 & Serine/threonine-protein phosphatase $2 \mathrm{~A} 65 \mathrm{kDa}$ regulatory subunit $\mathrm{AB}$. isoform & Z. mays & 12 & 136 & 5.03 & 66318 & protein \\
\hline 328 & B9N744 & Gamma class glutathione transferase EF1Bgamma3 & P. trichocarpa & 13 & 292 & 6.05 & 48353 & protein \\
\hline 329 & C5WQZ5 & Putative uncharacterized protein $\mathrm{Sb} 01 \mathrm{~g} 041210$ & S. bicolor & 3 & 155 & 7.65 & 51802 & protein \\
\hline 330 & E4MVY3 & mRNA, clone: RTFL01-01-M18 & T. halophila & 54 & 1191 & 6.25 & 94734 & protein \\
\hline 331 & E5GBS0 & 40 S ribosomal protein $\mathrm{S} 24$ & C. melo & 2 & 93 & 10.64 & 15761 & protein \\
\hline 332 & E5LCX1 & C14 cysteine protease & S. demissum & 2 & 125 & 4.98 & 23974 & protein \\
\hline 333 & E5RDE1 & $26 \mathrm{~S}$ proteasome non-ATPase regulatory subunit & C. melo & 4 & 113 & 4.73 & 34256 & protein \\
\hline 334 & F2CRM6 & Predicted protein & H. vulgare & 2 & 163 & 5.52 & 121394 & protein \\
\hline 335 & F2D448 & Predicted protein & H. vulgare & 13 & 273 & 10.17 & 30607 & protein \\
\hline 336 & F2D861 & Predicted protein & H. vulgare & 6 & 245 & 10.43 & 15765 & protein \\
\hline 337 & F2DAU4 & Predicted protein & H. vulgare & 39 & 678 & 7.56 & 62696 & protein \\
\hline 338 & F2DLC2 & Proteasome subunit alpha type & H. vulgare & 6 & 300 & 4.68 & 26087 & protein \\
\hline 339 & F2EKH0 & Predicted protein & H. vulgare & 6 & 276 & 4.60 & 31771 & protein \\
\hline 340 & F8WLB7 & $40 \mathrm{~S}$ ribosomal protein $\mathrm{S} 3$ & C. unshiu & 10 & 130 & 9.63 & 20599 & protein \\
\hline 341 & H1ZY49 & Translation elongation factor 1-alpha & L. maackii & 33 & 467 & 5.00 & 11619 & protein \\
\hline 342 & H6VUT7 & Translation elongation factor & A. mongolicus & 12 & 168 & 4.63 & 25300 & protein \\
\hline 343 & I0J3D8 & Proteasome subunit B. type & A. halleri & 2 & 133 & 6.06 & 33367 & protein \\
\hline 344 & J9QAL9 & Ribosomal protein L2 & A. corniculatum & 13 & 343 & 10.23 & 21703 & protein \\
\hline 345 & K3XJZ1 & Uncharacterized protein & S. italica & 2 & 51 & 9.39 & 35932 & protein \\
\hline 346 & K3XX30 & Uncharacterized protein & S. italica & 30 & 630 & 5.07 & 47991 & protein \\
\hline 347 & $\mathrm{~K} 3 \mathrm{XX} 43$ & Uncharacterized protein & S. italica & 21 & 404 & 6.29 & 48119 & protein \\
\hline 348 & K3YR06 & Uncharacterized protein & S. italica & 11 & 356 & 6.16 & 64225 & protein \\
\hline 349 & K3YZ28 & Uncharacterized protein & S. italica & 2 & 90 & 10.59 & 15971 & protein \\
\hline 350 & K3ZVI3 & Uncharacterized protein & S. italica & 11 & 271 & 5.16 & 33393 & protein \\
\hline 351 & K4A6E4 & Uncharacterized protein & S. italica & 6 & 76 & 5.16 & 83215 & protein \\
\hline
\end{tabular}




\begin{tabular}{|c|c|c|c|c|c|c|c|c|}
\hline 352 & K4AAQ4 & Uncharacterized protein & S. italica & 7 & 204 & 10.56 & 44399 & protein \\
\hline 353 & Q0W9E2 & B. chaperonin 60 & S. commersonii & 12 & 340 & 7.27 & 63333 & protein \\
\hline 354 & Q1KUM7 & Putative uncharacterized protein & C. spinosa & 20 & 280 & 6.62 & 59525 & protein \\
\hline 355 & Q39986 & Cysteine proteinase & Hemerocallis & 3 & 98 & 5.50 & 40006 & protein \\
\hline 356 & Q8L994 & T-complex protein 1 subunit delta & A. thaliana & 13 & 238 & 7.68 & 58177 & protein \\
\hline 357 & Q9ATF4 & Ribosomal protein L33 & C. sativa & 5 & 148 & 10.58 & 12831 & protein \\
\hline 358 & Q9SZ27 & Putative aspartate-tRNA ligase & A. thaliana & 2 & 128 & 6.37 & 57768 & protein \\
\hline 359 & Q9XEW9 & Elongation factor 1-alpha & L. longiflorum & 45 & 411 & 9.07 & 49711 & protein \\
\hline 360 & A2Y926 & Proteasome subunit beta type & O. sativa & 5 & 75 & 5.48 & 26357 & protein \\
\hline 361 & E5GBL8 & ATP-dependent clp protease & C. melo & 18 & 173 & 7.23 & 103416 & protein \\
\hline 362 & F2CRZ5 & Predicted protein & H. vulgare & 3 & 52 & 5.52 & 49253 & protein \\
\hline 363 & F2CSM1 & Predicted protein & H. vulgare & 6 & 46 & 6.84 & 55969 & protein \\
\hline 364 & F2D9E0 & Predicted protein & H. vulgare & 3 & 102 & 11.14 & 28037 & protein \\
\hline 365 & F2DXI0 & Predicted protein & H. vulgare & 14 & 134 & 7.09 & 102090 & protein \\
\hline 366 & F2VXU4 & Arf2 & H. brasiliensis & 8 & 205 & 6.43 & 20724 & protein \\
\hline 367 & K3XLU5 & Uncharacterized protein & S. italica & 4 & 62 & 10.08 & 24413 & protein \\
\hline 368 & K3YHH7 & Uncharacterized protein & S. italica & 3 & 76 & 8.72 & 51287 & protein \\
\hline 369 & K3Z5F7 & Uncharacterized protein & S. italica & 3 & 120 & 6.67 & 58832 & protein \\
\hline 370 & K3ZDK9 & Uncharacterized protein & S. italica & 2 & 112 & & 15191 & protein \\
\hline 371 & K3ZWL4 & Uncharacterized protein & S. italica & 3 & 79 & 9.66 & 26013 & protein \\
\hline 372 & Q42912 & Serine/threonine-protein phosphatase & M. domestica & 2 & 94 & & 35419 & protein \\
\hline 373 & A1Z3W7 & eIF5A & R. chinensis & 6 & 156 & 5.99 & 17703 & protein \\
\hline 374 & A8IZZ4 & Bi-ubiquitin & C. reinhardtii & 20 & 47 & 6.06 & 17185 & protein \\
\hline 375 & A8JCE1 & Predicted protein & C. reinhardtii & 15 & 36 & 7.94 & 33842 & protein \\
\hline 376 & B3TLW5 & Ubiquitin-conjugating enzyme 1 & E. guineensis & 15 & 210 & 6.57 & 17280 & protein \\
\hline 377 & B3TM50 & $26 \mathrm{~S}$ proteasome regulatory particle non-ATPase subunit 8 & E. guineensis & 5 & 29 & 6.28 & 34814 & protein \\
\hline 378 & B4G194 & $40 \mathrm{~S}$ ribosomal protein $\mathrm{SA}$ & Z. mays & 11 & 39 & 5.08 & 33640 & protein \\
\hline 379 & B6SIC6 & Ubiquitin-conjugating enzyme E2 N & Z. mays & 16 & 200 & 7.34 & 17234 & protein \\
\hline 380 & $\mathrm{C} 8 \mathrm{CP} 48$ & Ubiquitin-conjugating enzyme variant & C. sinensis & 10 & 423 & 6.68 & 16734 & protein \\
\hline 381 & $\mathrm{D} 2 \mathrm{X} 5 \mathrm{~K} 2$ & DEAD-box RNA helicase-like protein & P. persica & 20 & 395 & 5.69 & 47191 & protein \\
\hline 382 & D7UPN0 & SEC13 family protein & L. japonicus & 8 & 53 & 6.01 & 32940 & protein \\
\hline 383 & E4MWV7 & mRNA, clone: RTFL01-09-M07 & T. halophila & 4 & 54 & 7.42 & 10792 & protein \\
\hline
\end{tabular}




\begin{tabular}{|c|c|c|c|c|c|c|c|c|}
\hline 384 & E5GBW6 & $26 \mathrm{~S}$ proteasome non-ATPase regulatory subunit & C. melo & 2 & 38 & 5.92 & 44456 & protein \\
\hline 385 & F2CTR2 & Predicted protein & H. vulgare & 8 & 84 & 6.99 & 59167 & protein \\
\hline 386 & F2CXV7 & Predicted protein & H. vulgare & 7 & 322 & 8.05 & 25809 & protein \\
\hline 387 & F2CYA6 & Predicted protein & H. vulgare & 66 & 826 & 6.06 & 17119 & protein \\
\hline 388 & F8WQA8 & S9 Tyrosyl aminopeptidase & R. sativus & 5 & 97 & 5.45 & 76789 & protein \\
\hline 389 & K3ZC75 & Uncharacterized protein & S. italica & 9 & 37 & 4.72 & 96887 & protein \\
\hline 390 & K3ZH11 & Uncharacterized protein & S. italica & 11 & 67 & 5.38 & 118046 & protein \\
\hline 391 & K3ZXB8 & Uncharacterized protein & S. italica & 4 & 44 & 8.60 & 19603 & protein \\
\hline 392 & K3ZXP5 & Uncharacterized protein & S. italica & 9 & 50 & 6.80 & 17359 & protein \\
\hline 393 & O23962 & Translation elongation factor-TU & G. $\max$ & 5 & 37 & 5.19 & 27275 & protein \\
\hline 394 & Q1KUM6 & Putative uncharacterized protein & C. spinosa & 4 & 66 & 4.81 & 45374 & protein \\
\hline 395 & Q40556 & Protein phosphatase $2 \mathrm{~A}$ & N. tabacum & 3 & 49 & 5.17 & 66079 & protein \\
\hline 396 & Q5ZF92 & Eukaryotic translation initiation factor $5 \mathrm{~A}-1$ & P. major & 8 & 111 & 5.57 & 17486 & protein \\
\hline 397 & Q9ZVY6 & $\mathrm{T} 25 \mathrm{~N} 20.17$ & A. thaliana & 3 & 56 & 5.58 & 90732 & protein \\
\hline 398 & I0CC95 & Type II peroxiredoxin & T. hispida & 2 & 50 & 8.31 & 24226 & redox \\
\hline 399 & A9PII8 & Putative uncharacterized protein & P. trichocarpa & 5 & 61 & 8.29 & 67342 & redox \\
\hline 400 & $\mathrm{O} 24511$ & Catalase & N. tabacum & 19 & 236 & 7.33 & 57225 & redox \\
\hline 401 & Q6ZXH7 & Putative ascorbate peroxidase & P. canadensis & 7 & 197 & 4.97 & 22506 & redox \\
\hline 402 & Q7XTK9 & Catalase & P. persica & 20 & 422 & 7.25 & 57262 & redox \\
\hline 403 & Q9FE12 & Peroxiredoxin & P. vulgaris & 10 & 178 & 5.33 & 28776 & redox \\
\hline 404 & K017G7 & Thioredoxin peroxidase & N. tabacum & 7 & 89 & 8.03 & 29972 & redox \\
\hline 405 & Q9FEM4 & Catalase & B. pendula & 5 & 99 & 5.48 & 17247 & redox \\
\hline 406 & A1BLP6 & Thioredoxin & M. truncatula & 10 & 55 & 6.16 & 12904 & redox \\
\hline 407 & A5Z1Y7 & Cytochrome b5 & M. domestica & 3 & 87 & 5.52 & 15243 & redox \\
\hline 408 & B4FPK8 & Cytochrome b5 & Z. mays & 6 & 156 & 5.30 & 14958 & redox \\
\hline 409 & C5Z0K4 & Putative uncharacterized protein Sb09g004370 & S. bicolor & 6 & 49 & 6.87 & 40310 & redox \\
\hline 410 & E4MVQ7 & mRNA, clone: RTFL01-03-C18 & T. halophila & 11 & 263 & 6.60 & 46631 & redox \\
\hline 411 & G0WP59 & Superoxide dismutase $[\mathrm{Cu}-\mathrm{Zn}]$ & W. somnifera & 17 & 157 & 5.64 & 15782 & redox \\
\hline 412 & G5DXK7 & Membrane steroid-binding protein & S. latifolia & 6 & 136 & 4.61 & 23493 & redox \\
\hline 413 & O65156 & Glutathione peroxidase & Z. aethiopica & 5 & 121 & 9.73 & 26916 & redox \\
\hline 414 & Q3HVN5 & Dehydroascorbate reductase & S. tuberosum & 5 & 137 & 6.54 & 23596 & redox \\
\hline 415 & Q5PY86 & NADH:cytochrome b5 reductase & V. fordii & 5 & 35 & 7.15 & 31341 & redox \\
\hline
\end{tabular}




\begin{tabular}{|c|c|c|c|c|c|c|c|c|}
\hline 416 & Q93YG1 & Monodehydroascorbate reductase & M. crystalL. & 28 & 142 & 6.81 & 51944 & redox \\
\hline 417 & Q9SAT7 & Superoxide dismutase $[\mathrm{Cu}-\mathrm{Zn}]$ & P. tremuloides & 14 & 101 & 5.82 & 15420 & redox \\
\hline 418 & A8IBE0 & Predicted protein & C. reinhardtii & 2 & 26 & 9.22 & 75068 & RNA \\
\hline 419 & E1Z5Z1 & Putative uncharacterized protein & C. variabilis & 7 & 64 & 7.27 & 63288 & RNA \\
\hline 420 & Q9LY25 & Putative uncharacterized protein T2I1_60 & A. thaliana & 10 & 43 & 6.40 & 115126 & RNA \\
\hline 421 & G5DWG4 & DEAD-box ATP-dependent RNA helicase & S. latifolia & 5 & 28 & 6.02 & 85314 & RNA \\
\hline 422 & Q8LG45 & Small nuclear ribonucleoprotein-associated protein & A. thaliana & 2 & 77 & 10.71 & 26994 & RNA \\
\hline 423 & A9P9A2 & Putative uncharacterized protein & P. trichocarpa & 10 & 109 & 9.36 & 46272 & RNA \\
\hline 424 & E5GBL0 & Short-chain dehydrogenase/reductase & C. melo & 4 & 38 & 6.89 & 108844 & RNA \\
\hline 425 & Q2LFC1 & AGO4-2 & N. benthamiana & 8 & 40 & 8.78 & 102331 & RNA \\
\hline 426 & Q8L5C2 & $110 \mathrm{kDa} 4 \mathrm{SNc}$-Tudor domain protein & P. sativum & 7 & 39 & 7.36 & 108600 & RNA \\
\hline 427 & Q40270 & RNA-binding protein & M. crystalL. & 5 & 73 & 4.79 & 32001 & RNA \\
\hline 428 & Q84P56 & TGB12K interacting protein 2 & N. tabacum & 14 & 80 & 4.55 & 37547 & RNA \\
\hline 429 & K3XY79 & Uncharacterized protein & S. italica & 3 & 42 & 5.22 & 37046 & secondary metabolism \\
\hline 430 & J9XLE5 & Putative isopentenyl diphosphate isomerase & O. europaea & 11 & 111 & 5.38 & 25822 & secondary metabolism \\
\hline 431 & A9ZMZ5 & Acetyl-CoA C-acetyltransferase & H. brasiliensis & 3 & 117 & 6.68 & 42039 & secondary metabolism \\
\hline 432 & B4FYM0 & Acetyl-CoA acetyltransferase, cytosolic 2 & Z. mays & 4 & 147 & 7.66 & 43450 & secondary metabolism \\
\hline 433 & K4A9K9 & Uncharacterized protein & S. italica & 2 & 41 & 5.83 & 52168 & secondary metabolism \\
\hline 434 & Q8GZR6 & GcpE & S. lycopersicum & 29 & 726 & 6.11 & 82805 & secondary metabolism \\
\hline 435 & A9ZMZ4 & Acetyl-CoA C-acetyltransferase & H. brasiliensis & 5 & 47 & 7.33 & 41798 & secondary metabolism \\
\hline 436 & A9ZN09 & 2-C-methyl-D-erythritol 4-phosphate cytidylyltransferase & H. brasiliensis & 10 & 48 & 7.28 & 34509 & secondary metabolism \\
\hline 437 & B6UDL5 & Hydroxymethylbutenyl 4-diphosphate synthase & Z. mays & 26 & 205 & 5.97 & 82691 & secondary metabolism \\
\hline 438 & B9UP05 & Hydroxymethylbutenyl diphosphate reductase & Oncidium & 3 & 56 & 6.20 & 52389 & secondary metabolism \\
\hline 439 & E4MW60 & mRNA, clone: RTFL01-03-M14 & T. halophila & 6 & 50 & 7.42 & 39457 & secondary metabolism \\
\hline 440 & Q84XR5 & GCPE protein & C. roseus & 31 & 239 & 5.92 & 82522 & secondary metabolism \\
\hline 441 & A8JGV6 & 14-3-3 protein & C. reinhardtii & 10 & 164 & 5.00 & 29667 & signalling \\
\hline 442 & E1ZPL7 & Putative uncharacterized protein & C. variabilis & 2 & 71 & 8.88 & 41498 & signalling \\
\hline 443 & B9ICC7 & Calnexin family protein & P. trichocarpa & 5 & 71 & 4.78 & 60802 & signalling \\
\hline 444 & A2V880 & G protein B.-subunit-like protein & N. tabacum & 14 & 405 & 7.09 & 16100 & signalling \\
\hline 445 & F2CQ27 & Predicted protein & H. vulgare & 5 & 83 & 6.66 & 25460 & signalling \\
\hline 446 & F2CT86 & Predicted protein & H. vulgare & 2 & 60 & 7.47 & 22104 & signalling \\
\hline 447 & Q75ZE0 & 14-3-3 e-1 protein & N. tabacum & 9 & 145 & 4.78 & 29479 & signalling \\
\hline
\end{tabular}




\begin{tabular}{|c|c|c|c|c|c|c|c|c|}
\hline 448 & K3ZUF9 & Uncharacterized protein & S. italica & 8 & 108 & 8.6 & 41440 & signalling \\
\hline 449 & Q4W5U7 & Calnexin-like protein & S. lycopersicum & 5 & 88 & 4.82 & 61338 & signalling \\
\hline 450 & A9P8Q7 & Predicted protein & P. trichocarpa & 62 & 802 & 4.84 & 28824 & signalling \\
\hline 451 & B4FB55 & Ras-related protein ARA-3 & Z. mays & 12 & 144 & 8.22 & 24038 & signalling \\
\hline 452 & F2CRF1 & Predicted protein & H. vulgare & 78 & 796 & 4.88 & 29361 & signalling \\
\hline 453 & I4DSV6 & 14-3-3 protein & M. polymorpha & 73 & 1074 & 4.69 & 29526 & signalling \\
\hline 454 & O65165 & 14-3-3 like protein & M. crystalL. & 2 & 162 & 4.26 & 4502 & signalling \\
\hline 455 & O81976 & 14-3-3 Protein & G. $\max$ & 56 & 1109 & 4.21 & 7619 & signalling \\
\hline 456 & P93785 & 14-3-3 protein & S. tuberosum & 74 & 1304 & 4.82 & 29014 & signalling \\
\hline 457 & Q40463 & NTGB2 & N. tabacum & 11 & 235 & 6.19 & 15827 & signalling \\
\hline 458 & Q41340 & Small GTP-binding protein & S. lycopersicum & 12 & 192 & 5.44 & 22775 & signalling \\
\hline 459 & Q6UNT4 & Putative GTP-binding protein & C. sativus & 14 & 59 & 5.19 & 23366 & signalling \\
\hline 460 & Q706C9 & GDP dissociation inhibitor & M. truncatula & 16 & 214 & 5.88 & 50211 & signalling \\
\hline 461 & Q75ZD6 & 14-3-3 h-1 protein & N. tabacum & 88 & 1312 & 4.78 & 29121 & signalling \\
\hline 462 & Q84RQ1 & Calcium-dependent protein kinase & S. oligorhiza & 2 & 125 & 7.23 & 61263 & signalling \\
\hline 463 & Q8LC80 & Putative calcium-binding protein, calreticulin & A. thaliana & 26 & 509 & 4.53 & 48385 & signalling \\
\hline 464 & K3YHL2 & Uncharacterized protein & S. italica & 6 & 85 & 9.42 & 48818 & stress \\
\hline 465 & B6UBQ9 & Ankyrin protein kinase-like & Z. mays & 11 & 178 & 9.03 & 68790 & stress \\
\hline 466 & F2DX25 & Predicted protein & H. vulgare & 27 & 446 & 5.06 & 80668 & stress \\
\hline 467 & B6EBD6 & Heat shock protein $90-2$ & G. $\max$ & 51 & 851 & 5.07 & 80392 & stress \\
\hline 468 & B6U0V6 & Endoplasmin & Z. mays & 17 & 367 & 4.97 & 92831 & stress \\
\hline 469 & D0EJY9 & Molecular chaperone Hsp90-3 & N. benthamiana & 38 & 732 & 5.02 & 80638 & stress \\
\hline 470 & E4MXI2 & mRNA, clone: RTFL01-39-D20 & T. halophila & 48 & 999 & 5.21 & 73890 & stress \\
\hline 471 & F2D269 & Predicted protein & H. vulgare & 17 & 158 & 5.04 & 73306 & stress \\
\hline 472 & Q08II7 & Heat shock protein 70 -like protein & L. sativa & 4 & 291 & 10.33 & 12539 & stress \\
\hline 473 & Q0MYQ7 & Germin-like protein 2 & $V$. vinifera & 2 & 122 & 8.41 & 22832 & stress \\
\hline 474 & Q4LDR0 & Heat shock protein & S. lycopersicum & 7 & 120 & 6.55 & 110564 & stress \\
\hline 475 & Q8LG68 & UDP-glucose 6-dehydrogenase & A. thaliana & 8 & 199 & 6.18 & 53591 & stress \\
\hline 476 & B6EBD7 & Heat shock protein $90-1$ & G. $\max$ & 22 & 194 & 5.02 & 80700 & stress \\
\hline 477 & F2D884 & Predicted protein & H. vulgare & 112 & 1862 & 5.19 & 71476 & stress \\
\hline 478 & K4A6U5 & Uncharacterized protein & S. italica & 51 & 854 & 5.34 & 71710 & stress \\
\hline 479 & O49045 & Heat shock 70 protein & S. oleracea & 9 & 251 & 5.67 & 72558 & stress \\
\hline
\end{tabular}




\begin{tabular}{|c|c|c|c|c|c|c|c|c|}
\hline 480 & Q8VYN9 & Putative uncharacterized protein At5g54430 & A. thaliana & 2 & 62 & 6.57 & 26414 & stress \\
\hline 481 & Q9MAM6 & Pyruvate dehydrogenase E1 component subunit alpha-3, chloroplastic & A. thaliana & 10 & 45 & 8.76 & 75745 & TCA \\
\hline 482 & Q9C6Z3 & Pyruvate dehydrogenase E1 component subunit beta-2, chloroplastic & A. thaliana & 6 & 95 & 6.35 & 44672 & TCA \\
\hline 483 & B4G1C9 & Dihydrolipoyllysine-residue acetyltransferase component of pyruvatedehydrogenase & Z. mays & 9 & 216 & 8.10 & 47858 & TCA \\
\hline 484 & Q9C8P0 & $\begin{array}{l}\text { Dihydrolipoyllysine-residue acetyltransferase component } 5 \text { of pyruvate dehydrogenase } \\
\text { complex, chloroplastic }\end{array}$ & A. thaliana & 9 & 276 & 8.78 & 48334 & TCA \\
\hline 485 & Q9SPB1 & Leghemoglobin reductase & V. unguiculata & 5 & 62 & 8.05 & 56030 & TCA \\
\hline 486 & K3YH48 & Uncharacterized protein & S. italica & 5 & 56 & 8.66 & 56052 & TCA \\
\hline 487 & B6SHD3 & Malate dehydrogenase & Z. mays & 8 & 81 & 8.32 & 42623 & TCA \\
\hline 488 & D3GQL1 & Aconitate hydratase 3 & C. clementina & 25 & 310 & 6.30 & 98669 & TCA \\
\hline 489 & D3GQL2 & Aconitate hydratase 2 & C. clementina & 48 & 519 & 6.54 & 98475 & TCA \\
\hline 490 & E4MX73 & mRNA, clone: RTFL01-10-N01 & T. halophila & 4 & 126 & 5.83 & 39684 & TCA \\
\hline 491 & F2DAP7 & Malic enzyme & H. vulgare & 8 & 122 & 5.78 & 69436 & TCA \\
\hline 492 & F2DGF3 & Predicted protein & H. vulgare & 24 & 641 & 6.81 & 115601 & TCA \\
\hline 493 & F2DIS4 & Malic enzyme & H. vulgare & 6 & 99 & 7.40 & 68411 & TCA \\
\hline 494 & K3XFR6 & Uncharacterized protein & S. italica & 17 & 286 & 7.46 & 66448 & TCA \\
\hline 495 & K3Y7B0 & Uncharacterized protein & S. italica & 25 & 151 & 8.70 & 48942 & TCA \\
\hline 496 & K3YG24 & Uncharacterized protein & S. italica & 30 & 496 & 7.31 & 107135 & TCA \\
\hline 497 & K4ACE3 & Uncharacterized protein & S. italica & 19 & 171 & 6.09 & 35803 & TCA \\
\hline 498 & O81609 & Nodule-enhanced malate dehydrogenase & P. sativum & 4 & 173 & 7.75 & 42106 & TCA \\
\hline 499 & Q93WQ1 & Dihydrolipoyl dehydrogenase & B. gymnorhiza & 8 & 82 & 7.17 & 54389 & TCA \\
\hline 500 & Q9AXR6 & ATP:citrate lyase & C. аппиит & 22 & 502 & 7.39 & 66376 & TCA \\
\hline 501 & Q9M2T8 & 2-oxoglutarate dehydrogenase, E1 subunit-like protein & A. thaliana & 19 & 377 & 7.14 & 114888 & TCA \\
\hline 502 & Q9M6B3 & Malate dehydrogenase & V. vinifera & 15 & 419 & 8.62 & 37137 & TCA \\
\hline 503 & Q9ZNX1 & NAD-dependent isocitrate dehydrogenase & N. tabacum & 3 & 98 & 7.52 & 40651 & TCA \\
\hline 504 & F2E797 & Predicted protein & H. vulgare & 7 & 127 & 5.72 & 47111 & TCA \\
\hline 505 & K3ZQC3 & Uncharacterized protein & S. italica & 5 & 111 & 7.23 & 115569 & TCA \\
\hline 506 & B6TCZ3 & Malate dehydrogenase & Z. mays & 24 & 514 & 7.74 & 41394 & TCA \\
\hline 507 & D9ZBV9 & Malate dehydrogenase & S. globosa & 18 & 286 & 4.92 & 13766 & TCA \\
\hline 508 & F2D4W6 & Malate dehydrogenase & H. vulgare & 82 & 1458 & 5.88 & 35920 & TCA \\
\hline 509 & F2DBE0 & Predicted protein & H. vulgare & 6 & 144 & 8.22 & 34595 & TCA \\
\hline 510 & I1LCM5 & Uncharacterized protein & G. $\max$ & 2 & 51 & 6.96 & 47008 & TCA \\
\hline 511 & K3XJN7 & Uncharacterized protein & S. italica & 37 & 468 & 7.85 & 35629 & TCA \\
\hline
\end{tabular}




\begin{tabular}{|c|c|c|c|c|c|c|c|c|}
\hline 512 & $\mathrm{O} 24135$ & Citrate synthase & N. tabacum & 3 & 89 & 7.85 & 52744 & TCA \\
\hline 513 & B6TPE4 & Glutamate-1-semialdehyde 2,1-aminomutase & Z. mays & 6 & 81 & 6.55 & 50223 & tetrapyrrole synthesis \\
\hline 514 & B9HRI3 & V-type proton ATPase subunit a & P. trichocarpa & 6 & 86 & 6.10 & 93462 & transport \\
\hline 515 & K3XXR2 & Uncharacterized protein & S. italica & 13 & 117 & 9.20 & 39036 & transport \\
\hline 516 & F2D0V3 & Predicted protein & H. vulgare & 11 & 116 & 9.61 & 32911 & transport \\
\hline 517 & K3Z3J3 & Uncharacterized protein & S. italica & 34 & 482 & 6.64 & 105687 & transport \\
\hline 518 & F2CQQ3 & Predicted protein & H. vulgare & 18 & 688 & 5.16 & 54310 & transport \\
\hline 519 & K3YQW5 & Uncharacterized protein & S. italica & 21 & 599 & 5.39 & 68637 & transport \\
\hline 520 & K3ZD99 & Uncharacterized protein & S. italica & 3 & 90 & 9.16 & 26325 & transport \\
\hline 521 & C9WSP8 & Plasma membrane intrinsic protein & H. brasiliensis & 2 & 167 & & 30978 & transport \\
\hline 522 & Q43106 & $\mathrm{H}(+)$-transporting ATPase & P. vulgaris & 16 & 262 & 7.21 & 104776 & transport \\
\hline 523 & Q9AVU8 & Putative vacuolar ATP Synthase subunit A & M. crystallinum & 13 & 314 & 5.21 & 69139 & transport \\
\hline 524 & Q9C5M0 & Mitochondrial dicarboxylate/tricarboxylate transporter DTC & A. thaliana & 6 & 70 & 9.23 & 32347 & transport \\
\hline 525 & A9CM22 & Voltage-dependent anion channel & N. tabacum & 10 & 126 & 8.32 & 29694 & transport \\
\hline 526 & B1NHU0 & Aquaporin PIP1-2 & G. hirsutum & 6 & 41 & 8.40 & 31129 & transport \\
\hline 527 & D7SS06 & Putative uncharacterized protein & V. vinifera & 10 & 254 & 5.38 & 68964 & transport \\
\hline 528 & Q0MX14 & Putative aquaporin & $V$. vinifera & 3 & 66 & 8.15 & 30470 & transport \\
\hline 529 & Q43796 & Inorganic pyrophosphatase & N. tabacum & 16 & 199 & 5.34 & 56198 & transport \\
\hline
\end{tabular}

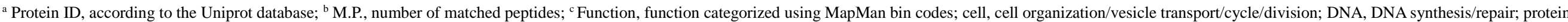

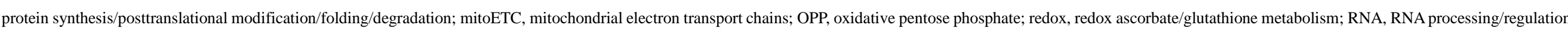
of transcription; TCA, tricarboxylic acid cycle; and misc, miscellaneous. 
Supplemental Table 13. Proteins Identified in Cerasus Flower Buds.

\begin{tabular}{|c|c|c|c|c|c|c|c|c|c|}
\hline No. & Protein ID ${ }^{\text {a }}$ & Description & Species & M.P. ${ }^{b}$ & Score & $\operatorname{Mol}(\%)^{\mathrm{c}}$ & $\mathrm{pI}$ & Mass (Da) & Function $^{\mathrm{d}}$ \\
\hline 1 & O81976 & 14-3-3 Protein & G. $\max$ & 21 & 797 & 1.94 & 4.21 & 7619 & signalling \\
\hline 2 & $\mathrm{O} 48874$ & Plasma membrane H+-ATPase & G. hirsutum & 6 & 291 & 1.85 & 7.25 & 7094 & transport \\
\hline 3 & B5M4B1 & Beta-tubulin & S. tuberosum & 87 & 1529 & 1.24 & 4.86 & 51166 & cell \\
\hline 4 & Q6QHU3 & Major cherry allergen Pru av 1.0201 & P. avium & 43 & 433 & 1.03 & 5.11 & 17397 & stress \\
\hline 5 & K3XG76 & Uncharacterized protein & S. italica & 111 & 2417 & 0.90 & 6.49 & 58945 & mitoETC \\
\hline 6 & C6GFP3 & ATP synthase subunit beta & G. hirsutum & 102 & 2113 & 0.88 & 6.29 & 59987 & mitoETC \\
\hline 7 & K3Z679 & Uncharacterized protein & S. italica & 95 & 965 & 0.86 & 4.76 & 50728 & cell \\
\hline 8 & A1BQW0 & Glyceraldehyde-3-phosphate dehydrogenase & N. attenuata & 87 & 1640 & 0.77 & 7.28 & 21799 & glycolysis \\
\hline 9 & C7SCX7 & eIF5A2 & P. deltoides & 13 & 218 & 0.74 & 5.99 & 17743 & protein \\
\hline 10 & B6ZAS7 & Vacuolar H+-ATPase subunit A & H. annuиs & 2 & 50 & 0.73 & 4.59 & 4224 & transport \\
\hline 11 & Q0ZUM8 & Beta-tubulin & M. viride & 58 & 538 & 0.70 & 5.46 & 45234 & cell \\
\hline 12 & Q7XAP7 & Glyceraldehyde-3-phosphate dehydrogenase & H. cordata & 50 & 1070 & 0.66 & 8.44 & 24077 & glycolysis \\
\hline 13 & B4FRM7 & $60 \mathrm{~S}$ ribosomal protein $\mathrm{L} 12$ & Z. mays & 14 & 243 & 0.65 & 8.95 & 17821 & protein \\
\hline 14 & E5GBV9 & Glyceraldehyde-3-phosphate dehydrogenase & C. melo & 74 & 1109 & 0.63 & 6.61 & 36471 & glycolysis \\
\hline 15 & D7NHW9 & 2-phospho-D-glycerate hydrolase & P. trifoliata & 68 & 1368 & 0.60 & 5.78 & 47986 & glycolysis \\
\hline 16 & A7KQH0 & Beta-tubulin & E. grandis & 74 & 642 & 0.59 & 4.94 & 50615 & cell \\
\hline 17 & Q1KUS3 & Putative uncharacterized protein & C. spinosa & 37 & 735 & 0.57 & 4.7 & 30545 & signalling \\
\hline 18 & Q41133 & Biotin carboxylase & R. communis & 9 & 116 & 0.48 & 7.56 & 13848 & lipid metabolism \\
\hline 19 & B9ZZE4 & Glyceraldehyde-3-phosphate dehydrogenase & I. nil & 88 & 1858 & 0.46 & 7.24 & 37071 & glycolysis \\
\hline 20 & Q0GY39 & UDP-glucose pyrophosphorylase & P. armeniaca & 17 & 277 & 0.45 & 7.05 & 20814 & glycolysis \\
\hline 21 & Q52RG9 & Peptidyl-prolyl cis-trans isomerase & S. tuberosum & 9 & 191 & 0.42 & 8.47 & 12829 & cell \\
\hline 22 & E5GBD5 & $60 \mathrm{~S}$ ribosomal protein 19 & C. melo & 8 & 175 & 0.42 & 9.45 & 21994 & protein \\
\hline 23 & E4MXI2 & mRNA, clone: RTFL01-39-D20 & T. halophila & 47 & 1110 & 0.41 & 5.21 & 73890 & stress \\
\hline 24 & B2XBQ5 & Mandelonitrile glucosyltransferase UGT85A19 & P. dulcis & 49 & 772 & 0.41 & 5.15 & 54175 & misc \\
\hline 25 & Q76KV5 & S-adenosylmethionine synthase & P. sativum & 27 & 407 & 0.38 & 6.76 & 37838 & amino acid metabolism \\
\hline 26 & Q32513 & Ribulosebiphosphate Carboxylase large subunit & L. coreana & 20 & 286 & 0.37 & 6.76 & 45993 & photosynthesis \\
\hline 27 & J7MPY1 & ATP synthase subunit alpha & M. domestica & 33 & 235 & 0.35 & 6.23 & 55600 & mitoETC \\
\hline 28 & D6PNH2 & AT1G18080-like protein & N. paniculata & 8 & 125 & 0.35 & 8.29 & 20212 & development \\
\hline 29 & K3YS05 & Uncharacterized protein & S. italica & 25 & 345 & 0.34 & 5.99 & 54002 & amino acid metabolism \\
\hline 30 & F2D4W6 & Malate dehydrogenase & H. vulgare & 41 & 954 & 0.34 & 5.88 & 35920 & TCA \\
\hline 31 & G4XN32 & Aquaporin PIP1 & M. prunifolia & 15 & 333 & 0.34 & 9.31 & 30913 & transport \\
\hline
\end{tabular}




\begin{tabular}{|c|c|c|c|c|c|c|c|c|c|}
\hline 32 & F2EG92 & Predicted protein & H. vulgare & 2 & 58 & 0.33 & 12.12 & 7602 & protein \\
\hline 33 & B6EBD7 & Heat shock protein $90-1$ & G. $\max$ & 35 & 554 & 0.32 & 5.02 & 80700 & stress \\
\hline 34 & K3Z675 & Uncharacterized protein & S. italica & 39 & 216 & 0.32 & 9.07 & 49563 & protein \\
\hline 35 & Q9LD48 & 40S ribosomal protein $\mathrm{S} 15$ & A. thaliana & 10 & 162 & 0.30 & 11.3 & 8134 & protein \\
\hline 36 & F2CTJ0 & Predicted protein & H. vulgare & 26 & 450 & 0.30 & 5.2 & 50525 & cell \\
\hline 37 & P93787 & 14-3-3 protein & S. tuberosum & 14 & 284 & 0.30 & 4.82 & 28354 & signalling \\
\hline 38 & J9PX40 & Enolase & P. americana & 35 & 986 & 0.30 & 5.57 & 48418 & glycolysis \\
\hline 39 & B6CQU8 & Profilin & P. dulcis & 11 & 244 & 0.30 & 4.84 & 14166 & cell \\
\hline 40 & Q40203 & RAB1C & L. japonicus & 13 & 305 & 0.30 & 5.44 & 22847 & signalling \\
\hline 41 & O48906 & Malate dehydrogenase & M. sativa & 32 & 420 & 0.29 & 7.97 & 43498 & TCA \\
\hline 42 & A0MQ91 & Phenylalanine ammonia-lyase & P. armeniaca & 5 & 115 & 0.29 & 6.54 & 32292 & secondary metabolism \\
\hline 43 & D6C638 & Ribulose-1,5-bisphosphate carboxylase/oxygenase large subunit & Psychotria & 14 & 258 & 0.28 & 6.58 & 50770 & photosynthesis \\
\hline 44 & B3TLQ5 & $60 \mathrm{~S}$ ribosomal protein L11 & E. guineensis & 5 & 162 & 0.28 & 9.92 & 20950 & protein \\
\hline 45 & K4ACE3 & Uncharacterized protein & S. italica & 37 & 859 & 0.28 & 6.09 & 35803 & TCA \\
\hline 46 & D7SS06 & Putative uncharacterized protein & V. vinifera & 23 & 399 & 0.27 & 5.38 & 68964 & transport \\
\hline 47 & K3XWW9 & Uncharacterized protein & S. italica & 41 & 662 & 0.26 & 5.31 & 48128 & glycolysis \\
\hline 48 & В6Т3Р9 & Enolase & Z. mays & 30 & 661 & 0.25 & 5.82 & 48386 & glycolysis \\
\hline 49 & A1YUL9 & Importin subunit alpha & N. benthamiana & 22 & 487 & 0.25 & 5.38 & 59114 & protein \\
\hline 50 & $\mathrm{~F} 2 \mathrm{CS} 54$ & Predicted protein & H. vulgare & 28 & 615 & 0.25 & 5.21 & 73301 & stress \\
\hline 51 & E5GBF5 & Pyridoxal biosynthesis lyase PdxS & C. melo & 11 & 349 & 0.24 & 6.18 & 33234 & co-factor and vitamine metabolism \\
\hline 52 & E4MX47 & mRNA, clone: RTFL01-10-J19 & T. halophila & 16 & 269 & 0.24 & 9.77 & 41718 & transport \\
\hline 53 & B6CBQ9 & Ribosomal protein S13 & G. $\max$ & 3 & 45 & 0.23 & 10.36 & 17187 & protein \\
\hline 54 & C7SCX8 & eIF5A3 & P. deltoides & 9 & 42 & 0.23 & 5.78 & 17612 & protein \\
\hline 55 & Q7M2B7 & Cytochrome-c oxidase chain II & Oenothera sp. & 4 & 140 & 0.23 & 6.49 & 10054 & mitoETC \\
\hline 56 & Q8MCY2 & Ribulose bisphosphate carboxylase large chain & V. baccifera & 15 & 198 & 0.23 & 6.64 & 48739 & not assigned \\
\hline 57 & Q8L4S4 & Phosphoglycerate kinase & A. speltoides & 17 & 145 & 0.23 & 5.01 & 31433 & photosynthesis \\
\hline 58 & D2KZ81 & S-adenosylmethionine synthase & M. domestica & 14 & 424 & 0.22 & 4.94 & 24831 & amino acid metabolism \\
\hline 59 & $\mathrm{C} 0 \mathrm{~K} 2 \mathrm{~V} 4$ & GDP-D-mannose pyrophosphorylase & R. nigrum & 8 & 218 & 0.22 & 7.06 & 28940 & cell wall \\
\hline 60 & Q8SF02 & Dicarboxylate/tricarboxylate carrier & N. tabacum & 12 & 162 & 0.22 & 9.44 & 32389 & transport \\
\hline 61 & E4MWB7 & mRNA, clone: RTFL01-04-D14 & T. halophila & 21 & 735 & 0.22 & 6.29 & 69655 & TCA \\
\hline 62 & B2VQE0 & Methionine synthase & O. ramosa & 53 & 823 & 0.22 & 6.4 & 84862 & amino acid metabolism \\
\hline 63 & A8MS28 & $60 \mathrm{~S}$ ribosomal protein $\mathrm{L} 27-3$ & A. thaliana & 2 & 58 & 0.22 & 10.15 & 15139 & protein \\
\hline
\end{tabular}




\begin{tabular}{|c|c|c|c|c|c|c|c|c|c|}
\hline 64 & Q8RVN2 & Class II chitinase & M. domestica & 2 & 146 & 0.21 & 7.24 & 11047 & stress \\
\hline 65 & K3Y885 & Uncharacterized protein & S. italica & 8 & 214 & 0.21 & 9.25 & 38281 & transport \\
\hline 66 & $\mathrm{O} 24106$ & RNA-binding protein & N. glutinosa & 4 & 132 & 0.20 & 5.66 & 15797 & RNA \\
\hline 67 & E1UHH0 & Plasma membrane ATPase 4 & M. balbisiana & 36 & 664 & 0.20 & 6.93 & 106079 & transport \\
\hline 68 & O48919 & Cytosolic ascorbate peroxidase & F. ananassa & 8 & 168 & 0.20 & 6.04 & 27374 & redox \\
\hline 69 & A0ZQC7 & ATP synthase subunit beta & E. hamiltonianus & 23 & 252 & 0.20 & 5.36 & 51418 & photosynthesis \\
\hline 70 & E4MWB5 & mRNA, clone: RTFL01-04-D06 & T. halophila & 33 & 498 & 0.19 & 6.67 & 85053 & amino acid metabolism \\
\hline 71 & Q8W594 & Prunasin hydrolase isoform $\mathrm{PH} \mathrm{C}$ & P. serotina & 16 & 258 & 0.19 & 5.8 & 61784 & misc \\
\hline 72 & Q9SGT4 & Elongation factor EF-2 & A. thaliana & 47 & 651 & 0.19 & 6.25 & 95098 & protein \\
\hline 73 & H9ZGE0 & Prunasin hydrolase & P. dulcis & 17 & 394 & 0.19 & 6.48 & 62281 & misc \\
\hline 74 & F2DSV2 & Predicted protein & H. vulgare & 16 & 222 & 0.19 & 9.8 & 41400 & transport \\
\hline 75 & Q84TK5 & Glutamate-1-semialdehyde 2,1-aminomutase & B. napus & 9 & 210 & 0.18 & 6.65 & 50632 & tetrapyrrole synthesis \\
\hline 76 & E5GCQ7 & Glutamate-1-semialdehyde 2,1-aminomutase & C. melo & 8 & 281 & 0.18 & 6.61 & 50339 & tetrapyrrole synthesis \\
\hline 77 & K3XXC5 & Uncharacterized protein & S. italica & 14 & 200 & 0.18 & 7.06 & 42409 & glycolysis \\
\hline 78 & A1X810 & $14-3-3 E$ & H. vulgare & 12 & 237 & 0.18 & 4.83 & 29533 & signalling \\
\hline 79 & C0LQ98 & Monodehydroascorbate reductase & M. domestica & 35 & 514 & 0.18 & 6.96 & 46997 & redox \\
\hline 80 & E0D8Z0 & Ribulose bisphosphate carboxylase large chain & Piptocarpha & 9 & 194 & 0.18 & 7.02 & 44514 & photosynthesis \\
\hline 81 & B5TGQ1 & Phospholipase D & P. persica & 26 & 384 & 0.18 & 6.34 & 92610 & lipid metabolism \\
\hline 82 & O81830 & Putative uncharacterized protein AT4g27270 & A. thaliana & 5 & 127 & 0.18 & 6.79 & 22355 & lipid metabolism \\
\hline 83 & O80415 & Mitochondrial phosphate transporter & A. thaliana & 6 & 186 & 0.17 & 9.19 & 31119 & transport \\
\hline 84 & Q4VPI0 & Major allergen Mal d 1.03F & M. domestica & 12 & 337 & 0.17 & 5.78 & 17625 & stress \\
\hline 85 & Q8LC80 & Putative calcium-binding protein, calreticulin & A. thaliana & 29 & 472 & 0.17 & 4.53 & 48385 & signalling \\
\hline 86 & E4MWK1 & mRNA, clone: RTFL01-14-N15 & T. halophila & 21 & 301 & 0.17 & 5.05 & 80304 & stress \\
\hline 87 & COSQK2 & Alpha expansin & Rosa & 6 & 93 & 0.17 & 9.17 & 26733 & cell wall \\
\hline 88 & Q42918 & Acetyl-CoA C-acyltransferase (3-ketoacyl-coa thiolase b) & M. indica & 14 & 343 & 0.17 & 8.21 & 45743 & amino acid metabolism \\
\hline 89 & K4A7N1 & Uncharacterized protein & S. italica & 19 & 193 & 0.16 & 6.02 & 61055 & protein \\
\hline 90 & K4A8Y9 & Uncharacterized protein & S. italica & 4 & 203 & 0.16 & 7.61 & 53531 & TCA \\
\hline 91 & Q9LM03 & Methionine synthase & S. tuberosum & 49 & 494 & 0.16 & 6.32 & 84898 & amino acid metabolism \\
\hline 92 & B5B3T4 & Fructose-bisphosphate aldolase & S. portulacastrum & 11 & 296 & 0.16 & 6.93 & 38685 & glycolysis \\
\hline 93 & Q53U38 & Similar to $60 \mathrm{~S}$ ribosomal protein $\mathrm{L} 35$ & S. lycopersicum & 7 & 185 & 0.16 & 10.81 & 14259 & protein \\
\hline 94 & E4MY36 & mRNA, clone: RTFL01-37-B16 & T. halophila & 16 & 420 & 0.16 & 6.25 & 63555 & glycolysis \\
\hline 95 & Q0W9E2 & Beta chaperonin 60 & S. commersonii & 16 & 445 & 0.16 & 7.27 & 63333 & protein \\
\hline
\end{tabular}




\begin{tabular}{|c|c|c|c|c|c|c|c|c|c|}
\hline 96 & K3YR06 & Uncharacterized protein & S. italica & 9 & 384 & 0.15 & 6.16 & 64225 & protein \\
\hline 97 & Q9M7E4 & Elongation factor 1-alpha & Z. mays & 16 & 65 & 0.15 & 9.29 & 49692 & protein \\
\hline 98 & O65744 & GDP dissociation inhibitor & C. arietinum & 17 & 249 & 0.15 & 5.97 & 50014 & signalling \\
\hline 99 & B9T1G7 & $\mathrm{H}(1+)$-transporting atpase plant/fungi plasma membrane type & R. communis & 26 & 320 & 0.15 & 6.81 & 105167 & transport \\
\hline 100 & Q43178 & $\mathrm{H}(+)$-transporting ATPase & S. tuberosum & 26 & 409 & 0.15 & 6.22 & 105458 & transport \\
\hline 101 & K3Z3J3 & Uncharacterized protein & S. italica & 30 & 547 & 0.15 & 6.64 & 105687 & transport \\
\hline 102 & B5TM95 & NADH dehydrogenase subunit 7 & A. thaliana & 6 & 197 & 0.15 & 7.12 & 45555 & mitoETC \\
\hline 103 & K3XFR6 & Uncharacterized protein & S. italica & 15 & 195 & 0.15 & 7.46 & 66448 & TCA \\
\hline 104 & A8MQR4 & $60 \mathrm{~S}$ acidic ribosomal protein $\mathrm{P} 0-2$ & A. thaliana & 6 & 167 & 0.15 & 4.84 & 30656 & protein \\
\hline 105 & D0UXW7 & Superoxide dismutase $[\mathrm{Cu}-\mathrm{Zn}]$ & B. oldhamii & 5 & 83 & 0.15 & 6.1 & 15200 & redox \\
\hline 106 & F2D269 & Predicted protein & H. vulgare & 10 & 113 & 0.14 & 5.04 & 73306 & stress \\
\hline 107 & E1ZFL5 & Putative uncharacterized protein & C. variabilis & 6 & 44 & 0.14 & 11.28 & 15659 & protein \\
\hline 108 & I6N9H0 & Ribosomal protein $\mathrm{S} 12$ & G. biloba & 4 & 33 & 0.14 & 11.12 & 15535 & protein \\
\hline 109 & C0LQA1 & GDP-D-mannose-3',5'-epimerase & M. domestica & 12 & 156 & 0.14 & 6.7 & 42854 & redox \\
\hline 110 & E5GBS0 & 40S ribosomal protein S24 & C. melo & 2 & 120 & 0.14 & 10.64 & 15761 & protein \\
\hline 111 & F2EHF8 & Triosephosphate isomerase & H. vulgare & 19 & 92 & 0.14 & 5.47 & 26990 & glycolysis \\
\hline 112 & G9MA91 & Fructose-bisphosphate aldolase & L. grandiflorum & 6 & 245 & 0.14 & 6.28 & 37690 & glycolysis \\
\hline 113 & B9N744 & Gamma class glutathione transferase EF1Bgamma3 & P. trichocarpa & 6 & 219 & 0.14 & 6.05 & 48353 & protein \\
\hline 114 & Q6T379 & Triosephosphate isomerase & S. chacoense & 14 & 278 & 0.14 & 5.99 & 27251 & glycolysis \\
\hline 115 & $\mathrm{O} 48903$ & Malate dehydrogenase & M. sativa & 9 & 271 & 0.13 & 8.29 & 38397 & gluconeogenesis \\
\hline 116 & Q9SJQ9 & Fructose-bisphosphate aldolase & A. thaliana & 8 & 183 & 0.13 & 7.39 & 38705 & glycolysis \\
\hline 117 & O04975 & Fructose-bisphosphate aldolase & M. crystallinum & 10 & 225 & 0.13 & 6.92 & 38362 & glycolysis \\
\hline 118 & I1JI00 & Uncharacterized protein & G. $\max$ & 4 & 92 & 0.13 & 9.33 & 28056 & cell wall \\
\hline 119 & B6STE5 & Peptidyl-prolyl cis-trans isomerase & Z. mays & 4 & 72 & 0.13 & 8.79 & 16531 & cell \\
\hline 120 & Q4W713 & Alpha-L-arabinofuranosidase / beta-D-xylosidase & P. pyrifolia & 41 & 896 & 0.13 & 8.38 & 84936 & cell wall \\
\hline 121 & B3TM39 & Ribosomal protein S14 & E. guineensis & 5 & 65 & 0.13 & 10.7 & 16428 & protein \\
\hline 122 & F2D9E0 & Predicted protein & H. vulgare & 6 & 196 & 0.13 & 11.14 & 28037 & protein \\
\hline 123 & A9PEX0 & Putative uncharacterized protein & P. trichocarpa & 17 & 168 & 0.13 & 6.92 & 40127 & not assigned \\
\hline 124 & Q3LAG5 & Cysteine synthase & N. tabacum & 3 & 61 & 0.13 & 5.83 & 34331 & amino acid metabolism \\
\hline 125 & A2TEI9 & Xyloglucan endotransglycosylase/hydrolase XTH-27 & P. trichocarpa & 11 & 296 & 0.13 & 8.03 & 34301 & cell wall \\
\hline 126 & D5HP43 & Xyloglucan endotransglucosylase/hydrolase 1 & F. chiloensis & 11 & 195 & 0.13 & 8.59 & 34493 & cell wall \\
\hline 127 & G5DVX2 & Phosphoglycerate kinase & S. latifolia & 10 & 230 & 0.13 & 6.58 & 51352 & photosynthesis \\
\hline
\end{tabular}




\begin{tabular}{|c|c|c|c|c|c|c|c|c|c|}
\hline 128 & Q9T030 & $\mathrm{NAD}(\mathrm{P}) \mathrm{H}$ oxidoreductase, isoflavone reductase-like protein & A. thaliana & 13 & 226 & 0.13 & 6.8 & 34222 & secondary metabolism \\
\hline 129 & A8MR07 & Pyruvate kinase & A. thaliana & 7 & 108 & 0.13 & 6.25 & 52256 & glycolysis \\
\hline 130 & K4A6V8 & Uncharacterized protein & S. italica & 12 & 210 & 0.13 & 7.03 & 70549 & glycolysis \\
\hline 131 & B6CQS1 & Putative allergen Pru du 1.03 & P. dulcis & 8 & 454 & 0.13 & 5.59 & 17231 & stress \\
\hline 132 & B6T834 & 40S ribosomal protein $\mathrm{S} 3 \mathrm{a}$ & Z. mays & 8 & 166 & 0.12 & 9.83 & 29947 & protein \\
\hline 133 & F2E397 & Predicted protein & H. vulgare & 9 & 199 & 0.12 & 5.87 & 34933 & major $\mathrm{CHO}$ metabolism \\
\hline 134 & Q7XAE2 & Putative fructokinase 2 & P. integrifolia & 13 & 240 & 0.12 & 5.35 & 35181 & major $\mathrm{CHO}$ metabolism \\
\hline 135 & K3YAF8 & Uncharacterized protein & S. italica & 4 & 133 & 0.12 & 5.67 & 17343 & photosynthesis \\
\hline 136 & G5DXN1 & Malic enzyme & S. latifolia & 7 & 168 & 0.12 & 5.85 & 35532 & TCA \\
\hline 137 & K3XFV5 & Uncharacterized protein & S. italica & 20 & 245 & 0.12 & 5.91 & 65866 & TCA \\
\hline 138 & E5GC94 & Fructokinase & C. melo & 9 & 188 & 0.12 & 5.83 & 35801 & major $\mathrm{CHO}$ metabolism \\
\hline 139 & K3XYP4 & Uncharacterized protein & S. italica & 5 & 79 & 0.12 & 5.88 & 30448 & protein \\
\hline 140 & C4PB36 & Calreticulin & C. papaya & 21 & 196 & 0.12 & 4.48 & 48438 & signalling \\
\hline 141 & E5GCD6 & Multicopper oxidase & C. melo & 15 & 148 & 0.12 & 9.32 & 60810 & not assigned \\
\hline 142 & Q8LFR4 & Glutamate decarboxylase, putative & A. thaliana & 11 & 212 & 0.12 & 5.66 & 56224 & amino acid metabolism \\
\hline 143 & G1JSH2 & At1g58380 & A. thaliana & 12 & 208 & 0.12 & 10.26 & 30980 & protein \\
\hline 144 & B1Q3F1 & Glutamate decarboxylase isoform2 & S. lycopersicum & 15 & 177 & 0.12 & 5.62 & 57263 & amino acid metabolism \\
\hline 145 & Q6TMX3 & Peptidyl-prolyl cis-trans isomerase & T. halophila & 4 & 77 & 0.12 & 8.43 & 18600 & cell \\
\hline 146 & D6PAY2 & Peptidyl-prolyl cis-trans isomerase & Vanda & 20 & 40 & 0.12 & 8.46 & 18582 & cell \\
\hline 147 & Q645M9 & Glyoxisomal malate dehydrogenase & S. lycopersicum & 6 & 112 & 0.12 & 7.94 & 38007 & gluconeogenesis \\
\hline 148 & В3TM36 & $40 \mathrm{~S}$ ribosomal protein $\mathrm{S} 8$ & E. guineensis & 4 & 148 & 0.12 & 10.43 & 25347 & protein \\
\hline 149 & K4A5Z8 & Uncharacterized protein & S. italica & 22 & 468 & 0.12 & 5.24 & 90338 & cell \\
\hline 150 & Q6SKP4 & Ribosomal protein L3 & S. lycopersicum & 10 & 104 & 0.11 & 10.08 & 44836 & protein \\
\hline 151 & E5GCD7 & Succinate dehydrogenase & C. melo & 4 & 110 & 0.11 & 8.44 & 31941 & TCA \\
\hline 152 & Q5QGZ8 & Nitrilase 4A & L. angustifolius & 8 & 289 & 0.11 & 5.57 & 38080 & misc \\
\hline 153 & Q8W132 & Serine carboxypeptidase & N. pseudonarcissus & 3 & 39 & 0.11 & 6.99 & 19157 & protein \\
\hline 154 & K3ZRM5 & Uncharacterized protein & S. italica & 13 & 311 & 0.11 & 5.44 & 65858 & stress \\
\hline 155 & F2D653 & Predicted protein & H. vulgare & 4 & 85 & 0.11 & 6.02 & 33393 & amino acid metabolism \\
\hline 156 & O78327 & Transketolase 1 & C. аппиит & 12 & 167 & 0.11 & 6.62 & 80398 & OPP \\
\hline 157 & Q9SF16 & AT3g11830/F26K24_12 & A. thaliana & 6 & 280 & 0.11 & 6.39 & 60195 & protein \\
\hline 158 & B9SIJ9 & O-methyltransferase, putative & R. communis & 8 & 111 & 0.11 & 5.68 & 40259 & secondary metabolism \\
\hline 159 & A5JPK7 & Monodehydroascorbate reductase & $V$. vinifera & 20 & 225 & 0.11 & 5.93 & 47478 & redox \\
\hline
\end{tabular}




\begin{tabular}{|c|c|c|c|c|c|c|c|c|c|}
\hline 160 & B8AWV4 & Putative uncharacterized protein & O. sativa & 5 & 246 & 0.10 & 6.43 & 62652 & amino acid metabolism \\
\hline 161 & B6T9G3 & Alpha-1,4-glucan-protein synthase 1 & Z. mays & 20 & 388 & 0.10 & 7.05 & 41278 & cell wall \\
\hline 162 & Q1KV01 & Putative uncharacterized protein & C. spinosa & 16 & 365 & 0.10 & 5.95 & 41768 & cell wall \\
\hline 163 & Q30E95 & Type II SK2 dehydrin & P. persica & 8 & 69 & 0.10 & 5.48 & 28525 & stress \\
\hline 164 & C8YNG6 & 3-ketoacyl CoA thiolase 1 & P. hybrida & 10 & 258 & 0.10 & 8.29 & 49405 & amino acid metabolism \\
\hline 165 & B3TLT9 & $60 \mathrm{~S}$ ribosomal protein $\mathrm{L} 7$ & E. guineensis & 10 & 38 & 0.10 & 10.1 & 28665 & protein \\
\hline 166 & D7LW57 & Ketol-acid reductoisomerase & A. lyrata & 9 & 455 & 0.10 & 6.8 & 64346 & amino acid metabolism \\
\hline 167 & Q8LGJ6 & Phosphoglycerate dehydrogenase-like protein & A. thaliana & 19 & 170 & 0.10 & 6.76 & 63556 & amino acid metabolism \\
\hline 168 & O50000 & Abscisic stress ripening protein homolog & P. armeniaca & 4 & 232 & 0.10 & 6.05 & 21228 & not assigned \\
\hline 169 & K3ZX77 & Uncharacterized protein & S. italica & 2 & 77 & 0.10 & 11.34 & 21045 & protein \\
\hline 170 & I1W1U1 & Sucrose synthase & P. persica & 14 & 324 & 0.10 & 6.37 & 92946 & major $\mathrm{CHO}$ metabolism \\
\hline 171 & B0M199 & Peroxisomal fatty acid beta-oxidation multifunctional protein & G. $\max$ & 17 & 241 & 0.10 & 9.06 & 79273 & lipid metabolism \\
\hline 172 & B4F9G8 & Pyruvate kinase & Z. mays & 9 & 186 & 0.10 & 6.9 & 57820 & glycolysis \\
\hline 173 & A3QVW4 & Chloroplast glyceraldehyde-3-phosphate dehydrogenase & M. polymorpha & 6 & 157 & 0.10 & 7.47 & 43558 & photosynthesis \\
\hline 174 & K3XVH0 & Uncharacterized protein & S. italica & 14 & 238 & 0.10 & 6.62 & 80203 & photosynthesis \\
\hline 175 & Q945D3 & $26 \mathrm{~S}$ proteasome regulatory subunit S12 isolog-like protein & C. sativa & 2 & 78 & 0.10 & 5.12 & 21855 & protein \\
\hline 176 & E4MY28 & mRNA, clone: RTFL01-42-K21 & T. halophila & 2 & 41 & 0.10 & 9.11 & 21788 & signalling \\
\hline 177 & F2DYD4 & Predicted protein & H. vulgare & 3 & 71 & 0.10 & 6.35 & 21984 & signalling \\
\hline 178 & Q9AXR6 & ATP:citrate lyase & C. аппиит & 13 & 164 & 0.10 & 7.39 & 66376 & TCA \\
\hline 179 & A9CM21 & Voltage-dependent anion channel & N. tabacum & 14 & 102 & 0.10 & 8.07 & 29399 & transport \\
\hline 180 & B9T501 & $\mathrm{H}(+)$-transporting atpase plant/fungi plasma membrane type, putative & R. communis & 17 & 113 & 0.10 & 8.18 & 82006 & transport \\
\hline 181 & O65449 & At4g22010 & A. thaliana & 12 & 118 & 0.09 & 9.89 & 60589 & misc \\
\hline 182 & Q8L9B9 & Chloroplast nucleoid DNA binding protein, putative & A. thaliana & 3 & 34 & 0.09 & 9.23 & 53080 & RNA \\
\hline 183 & O81413 & Dihydrolipoyl dehydrogenase & G. $\max$ & 9 & 208 & 0.09 & 7.34 & 53311 & TCA \\
\hline 184 & Q8LG68 & UDP-glucose 6-dehydrogenase & A. thaliana & 7 & 226 & 0.09 & 6.18 & 53591 & cell wall \\
\hline 185 & K3ZGW5 & Uncharacterized protein & S. italica & 33 & 431 & 0.09 & 5.39 & 194875 & cell \\
\hline 186 & Q9LF33 & UDP-glucose 6-dehydrogenase & A. thaliana & 9 & 326 & 0.09 & 6.04 & 53653 & cell wall \\
\hline 187 & C0J025 & Peptidyl-prolyl cis-trans isomerase & T. aestivum & 6 & 90 & 0.09 & 9.57 & 22954 & cell \\
\hline 188 & B6SPZ8 & Uncharacterized protein & Z. mays & 4 & 94 & 0.09 & 7.91 & 23348 & cell \\
\hline 189 & Q6UNT3 & Hypersensitive-induced response protein & C. sativus & 3 & 78 & 0.09 & 5.33 & 31452 & not assigned \\
\hline 190 & Q9FM97 & Pyruvate kinase & A. thaliana & 7 & 77 & 0.09 & 6.86 & 55060 & glycolysis \\
\hline 191 & K3XFX7 & Uncharacterized protein & S. italica & 7 & 149 & 0.09 & 6.68 & 64026 & glycolysis \\
\hline
\end{tabular}




\begin{tabular}{|c|c|c|c|c|c|c|c|c|c|}
\hline 192 & Q5G1U0 & Malic enzyme & Z. mays & 20 & 249 & 0.09 & 7.77 & 71934 & TCA \\
\hline 193 & F1T1E9 & Chaperone protein HtpG & C. braunii & 13 & 217 & 0.09 & 5.15 & 80715 & stress \\
\hline 194 & I1U4K5 & Polyphenol oxidase IVa & M. domestica & 9 & 381 & 0.09 & 6.84 & 65582 & not assigned \\
\hline 195 & Q9AYS1 & Beta-galactosidase & P. pyrifolia & 12 & 90 & 0.09 & 6.34 & 81555 & misc \\
\hline 196 & Q9ZNX1 & NAD-dependent isocitrate dehydrogenase & N. tabacum & 4 & 134 & 0.09 & 7.52 & 40651 & TCA \\
\hline 197 & K3Y9Y1 & Uncharacterized protein & S. italica & 2 & 68 & 0.09 & 10.07 & 24396 & protein \\
\hline 198 & B3TLY3 & Ribosomal protein L15 & E. guineensis & 2 & 137 & 0.09 & 11.49 & 24371 & protein \\
\hline 199 & K3Z566 & Uncharacterized protein & S. italica & 5 & 134 & 0.08 & 7.77 & 59005 & C1-metabolism \\
\hline 200 & A9RZ64 & Predicted protein & P.patens & 5 & 73 & 0.08 & 7.87 & 50746 & amino acid metabolism \\
\hline 201 & H6VUT7 & Translation elongation factor & A. mongolicus & 6 & 143 & 0.08 & 4.63 & 25300 & protein \\
\hline 202 & F2CSZ4 & Predicted protein & H. vulgare & 2 & 40 & 0.08 & 4.58 & 25458 & RNA \\
\hline 203 & F2D1P1 & Predicted protein & H. vulgare & 10 & 101 & 0.08 & 9.31 & 34416 & protein \\
\hline 204 & Q9LZ82 & AT5g04430/T32M21_30 & A. thaliana & 3 & 49 & 0.08 & 6.01 & 33857 & RNA \\
\hline 205 & F6HXK8 & Pectinesterase & $V$. vinifera & 9 & 124 & 0.08 & 8.54 & 69782 & cell wall \\
\hline 206 & D4N5G0 & Alpha-form rubisco activase & G. $\max$ & 8 & 129 & 0.08 & 6.25 & 52531 & photosynthesis \\
\hline 207 & $\mathrm{O} 24135$ & Citrate synthase & N. tabacum & 8 & 285 & 0.08 & 7.85 & 52744 & TCA \\
\hline 208 & G4XN37 & Aquaporin TIP1 & M. prunifolia & 4 & 57 & 0.08 & 5.68 & 26054 & transport \\
\hline 209 & K3YG24 & Uncharacterized protein & S. italica & 21 & 113 & 0.08 & 6.89 & 107135 & TCA \\
\hline 210 & K3Z913 & Uncharacterized protein & S. italica & 4 & 136 & 0.08 & 9.47 & 26793 & cell \\
\hline 211 & E5GCR9 & Methylmalonate-semialdehyde dehydrogenase & C. melo & 5 & 115 & 0.08 & 6.09 & 56147 & amino acid metabolism \\
\hline 212 & F6H740 & Putative uncharacterized protein & $V$. vinifera & 24 & 560 & 0.08 & 7.99 & 84234 & cell wall \\
\hline 213 & Q6RXY3 & Beta xylosidase & F. ananassa & 25 & 570 & 0.08 & 8.34 & 84347 & cell wall \\
\hline 214 & G5DXN4 & Oligosaccharyltransferase complex subunit beta & S. latifolia & 4 & 124 & 0.08 & 5.43 & 27704 & misc \\
\hline 215 & A6XMY8 & Methylenetetrahydrofolate reductase & T. monococcum & 11 & 105 & 0.07 & 6.2 & 65461 & C1-metabolism \\
\hline 216 & K3Y8G3 & Uncharacterized protein & S. italica & 7 & 95 & 0.07 & 5.58 & 37785 & nucleotide metabolism \\
\hline 217 & D8FSK3 & Aquaporin PIP2;4 & G. hirsutum & 6 & 77 & 0.07 & 9 & 29061 & transport \\
\hline 218 & K3ZTC4 & Uncharacterized protein & S. italica & 4 & 178 & 0.07 & 6.73 & 48904 & misc \\
\hline 219 & K3XYW1 & Uncharacterized protein & S. italica & 6 & 122 & 0.07 & 10.32 & 29438 & protein \\
\hline 220 & Q8LCM9 & Putative uncharacterized protein & A. thaliana & 7 & 290 & 0.07 & 7.71 & 39873 & not assigned \\
\hline 221 & F2D927 & Predicted protein & H. vulgare & 9 & 66 & 0.07 & 5.2 & 80638 & transport \\
\hline 222 & Q39834 & Clathrin heavy chain & G. $\max$ & 23 & 308 & 0.07 & 5.55 & 194430 & cell \\
\hline 223 & K3ZHY5 & Uncharacterized protein & S. italica & 7 & 50 & 0.07 & 5.43 & 59383 & protein \\
\hline
\end{tabular}




\begin{tabular}{|c|c|c|c|c|c|c|c|c|c|}
\hline 224 & Q9FZG9 & Putative pectinesterase & A. thaliana & 11 & 169 & 0.07 & 9.45 & 60580 & cell wall \\
\hline 225 & F2DEW5 & Predicted protein & H. vulgare & 10 & 284 & 0.07 & 5.47 & 61723 & photosynthesis \\
\hline 226 & B6TNX7 & Proteasome subunit alpha type & Z. mays & 4 & 123 & 0.07 & 5.4 & 30582 & protein \\
\hline 227 & K0I7G7 & Thioredoxin peroxidase & N. tabacum & 4 & 119 & 0.07 & 8.03 & 29972 & redox \\
\hline 228 & G9HVM7 & Dehydrin 1 & C. sinensis & 2 & 121 & 0.07 & 5.82 & 29994 & stress \\
\hline 229 & P93252 & Porin & M. crystallinum & 2 & 172 & 0.07 & 8.6 & 29551 & transport \\
\hline 230 & Q5DVU0 & Plasma membrane intrinsic protein $1 ; 1$ & M. pudica & 3 & 39 & 0.07 & 8.41 & 31055 & transport \\
\hline 231 & E4MXN9 & mRNA, clone: RTFL01-28-I17 & T. halophila & 13 & 56 & 0.07 & 5.85 & 51931 & glycolysis \\
\hline 232 & K3Y7X1 & Uncharacterized protein & S. italica & 4 & 112 & 0.07 & 6.4 & 41980 & photosynthesis \\
\hline 233 & A9ZMZ4 & Acetyl-CoA C-acetyltransferase & H. brasiliensis & 3 & 86 & 0.07 & 7.33 & 41798 & secondary metabolism \\
\hline 234 & Q8VXG8 & Phosphoenolpyruvate carboxylase & K. pinnata & 5 & 34 & 0.07 & 8.75 & 42203 & glycolysis \\
\hline 235 & Q9MAT0 & $26 \mathrm{~S}$ proteasome regulatory subunit $\mathrm{N} 2$ & A. thaliana & 10 & 56 & 0.07 & 5.35 & 109254 & protein \\
\hline 236 & Q8W4X6 & Lipoxygenase & P. dulcis & 11 & 225 & 0.06 & 5.8 & 97918 & hormone metabolism \\
\hline 237 & F2DQ10 & Predicted protein & H. vulgare & 11 & 199 & 0.06 & 5.19 & 98573 & protein \\
\hline 238 & Q9SM58 & Acetohydroxy acid isomeroreductase & P. sativum & 10 & 153 & 0.06 & 6.62 & 63155 & amino acid metabolism \\
\hline 239 & F6HGZ1 & Pectinesterase & V. vinifera & 4 & 102 & 0.06 & 7.49 & 63329 & cell wall \\
\hline 240 & $\mathrm{O} 22075$ & Acidic endochitinase & T. glabra & 2 & 38 & 0.06 & 8.63 & 33414 & stress \\
\hline 241 & F2CQ79 & 3-ketoacyl-CoA synthase & H. vulgare & 2 & 49 & 0.06 & 9.32 & 57056 & lipid metabolism \\
\hline 242 & I6YMA7 & Uncharacterized protein & L. usitatissimum & 7 & 180 & 0.06 & 6.39 & 89937 & co-factor and vitamine metabolism \\
\hline 243 & E1ZNW4 & Putative uncharacterized protein & C. variabilis & 3 & 40 & 0.06 & 7.74 & 33730 & co-factor and vitamine metabolism \\
\hline 244 & Q4L0W6 & Spermidine synthase & C. sativus & 4 & 48 & 0.06 & 5.06 & 34786 & polyamine metabolism \\
\hline 245 & K3ХKB6 & Uncharacterized protein & S. italica & 3 & 38 & 0.06 & 6.06 & 33579 & RNA \\
\hline 246 & Q7G1G6 & Aspartate aminotransferase & G. $\max$ & 9 & 75 & 0.06 & 7.88 & 45800 & amino acid metabolism \\
\hline 247 & A5AMY6 & Putative uncharacterized protein & V. vinifera & 12 & 71 & 0.06 & 5.96 & 59063 & protein \\
\hline 248 & K3XJZ1 & Uncharacterized protein & S. italica & 2 & 57 & 0.06 & 9.39 & 35932 & protein \\
\hline 249 & Q8H9C9 & 3-deoxy-D-arabino-heptulosonate 7-phosphate synthase & S. tuberosum & 8 & 42 & 0.06 & 8.85 & 60062 & amino acid metabolism \\
\hline 250 & K3ZR86 & Uncharacterized protein & S. italica & 8 & 143 & 0.06 & 9.01 & 74682 & fermentation \\
\hline 251 & F2CPZ4 & Acyl-coenzyme A oxidase & H. vulgare & 11 & 278 & 0.06 & 7.08 & 74458 & lipid metabolism \\
\hline 252 & F4YGN7 & Hexokinase 1 & H. vulgare & 3 & 80 & 0.06 & 5.94 & 36966 & major $\mathrm{CHO}$ metabolism \\
\hline 253 & O81299 & AT4g02340 protein & A. thaliana & 3 & 49 & 0.06 & 6.76 & 36805 & misc \\
\hline 254 & E4MXR5 & mRNA, clone: RTFL01-40-M04 & T. halophila & 4 & 218 & 0.06 & 5.9 & 73489 & stress \\
\hline 255 & F2EC99 & Predicted protein & H. vulgare & 8 & 64 & 0.06 & 9.51 & 60941 & not assigned \\
\hline
\end{tabular}




\begin{tabular}{|c|c|c|c|c|c|c|c|c|c|}
\hline 256 & A8I7T1 & Heat shock protein $90 \mathrm{~B}$ & C. reinhardtii & 7 & 219 & 0.06 & 4.88 & 87547 & stress \\
\hline 257 & Q9ZR53 & Annexin-like protein & M. sativa & 5 & 41 & 0.05 & 9.07 & 38139 & cell \\
\hline 258 & E4MW76 & mRNA, clone: RTFL01-03-P23 & T. halophila & 2 & 109 & 0.05 & 8.38 & 38469 & cell \\
\hline 259 & Q9LPD0 & F22M8.4 protein & A. thaliana & 2 & 53 & 0.05 & 4.81 & 38889 & transport \\
\hline 260 & I1UYB5 & Pectinesterase & C. arabica & 3 & 60 & 0.05 & 9.31 & 39662 & cell wall \\
\hline 261 & B9RI89 & Serine-threonine protein kinase, plant-type, putative & R. communis & 4 & 67 & 0.05 & 8.18 & 40375 & not assigned \\
\hline 262 & Q9SZ27 & Putative aspartate-tRNA ligase & A. thaliana & 2 & 56 & 0.05 & 6.37 & 57768 & protein \\
\hline 263 & B6TDJ2 & Putative uncharacterized protein & Z. mays & 3 & 142 & 0.05 & 6.55 & 41970 & amino acid metabolism \\
\hline 264 & O04946 & Enoyl-ACP reductase & N. tabacum & 5 & 32 & 0.05 & 8.78 & 41925 & lipid metabolism \\
\hline 265 & O65736 & Beta-galactosidase & C. arietinum & 7 & 97 & 0.05 & 8.24 & 81933 & misc \\
\hline 266 & Q9ZP30 & Beta-galactosidase & C. papaya & 10 & 85 & 0.05 & 8.03 & 81618 & misc \\
\hline 267 & Q9M8R4 & Class I glutamine amidotransferase-like domain-containing protein & A. thaliana & 4 & 51 & 0.05 & 5.41 & 42417 & not assigned \\
\hline 268 & E4MW02 & mRNA, clone: RTFL01-05-D24 & T. halophila & 6 & 128 & 0.05 & 7.36 & 41230 & protein \\
\hline 269 & Q9XF79 & Papain-like cysteine protease & S. aurantiaca & 8 & 345 & 0.05 & 7.08 & 41182 & protein \\
\hline 270 & K3XPZ4 & Uncharacterized protein & S. italica & 7 & 129 & 0.05 & 6.18 & 41692 & secondary metabolism \\
\hline 271 & Q43637 & Acetoacetyl-coenzyme A thiolase & R. sativus & 3 & 38 & 0.05 & 6.77 & 42507 & secondary metabolism \\
\hline 272 & $\mathrm{~A} 0 \mathrm{~A} 4 \mathrm{Y} 2$ & Malic enzyme & Z. mays & 4 & 109 & 0.05 & 6.92 & 71079 & TCA \\
\hline 273 & F2DEJ0 & Predicted protein & H. vulgare & 7 & 50 & 0.05 & 6.68 & 68490 & protein \\
\hline 274 & Q96327 & Cell cycle-related nuclear binding protein & A. thaliana & 4 & 52 & 0.05 & 6.8 & 43294 & protein \\
\hline 275 & O81340 & $26 \mathrm{~S}$ proteasome regulatory subunit S5A & M. crystallinum & 3 & 35 & 0.05 & 4.64 & 42960 & protein \\
\hline 276 & A9P9A2 & Putative uncharacterized protein & P. trichocarpa & 4 & 128 & 0.04 & 9.36 & 46272 & RNA \\
\hline 277 & Q8L934 & Nucleoid DNA-binding-like protein & A. thaliana & 4 & 34 & 0.04 & 9.09 & 46259 & RNA \\
\hline 278 & Q8LB37 & Mevalonate diphosphate decarboxylase & A. thaliana & 8 & 83 & 0.04 & 6.98 & 45041 & secondary metabolism \\
\hline 279 & Q8LBA3 & Phosphoserine aminotransferase & A. thaliana & 4 & 85 & 0.04 & 8.06 & 47739 & amino acid metabolism \\
\hline 280 & B9HXE1 & Predicted protein & P. trichocarpa & 2 & 44 & 0.04 & 4.96 & 47512 & cell wall \\
\hline 281 & K3ZR21 & Uncharacterized protein & S. italica & 5 & 191 & 0.04 & 7.56 & 79012 & C1-metabolism \\
\hline 282 & K4A6E4 & Uncharacterized protein & S. italica & 7 & 55 & 0.04 & 5.16 & 83215 & protein \\
\hline 283 & F2E7G1 & Predicted protein & H. vulgare & 3 & 86 & 0.04 & 6.21 & 49826 & protein \\
\hline 284 & E4MVV4 & mRNA, clone: RTFL01-03-G08 & T. halophila & 4 & 52 & 0.04 & 5.4 & 51398 & RNA \\
\hline 285 & G8HAB1 & PLP-dependent aminotransferase & P. somniferum & 3 & 59 & 0.04 & 7.09 & 50587 & secondary metabolism \\
\hline 286 & K3Z3U2 & Uncharacterized protein & S. italica & 11 & 166 & 0.04 & 5.07 & 93903 & stress \\
\hline 287 & Q8S531 & Cytosolic aldehyde dehydrogenase RF2C & Z. mays & 3 & 118 & 0.04 & 6.39 & 54331 & fermentation \\
\hline
\end{tabular}




\begin{tabular}{|c|c|c|c|c|c|c|c|c|c|}
\hline 288 & A8IVR6 & Pyruvate kinase & C. reinhardtii & 5 & 69 & 0.04 & 7.08 & 55233 & glycolysis \\
\hline 289 & H2DH15 & Cytochrome P450 CYP716A47 & P. ginseng & 5 & 46 & 0.04 & 9.1 & 55549 & misc \\
\hline 290 & F1LKN0 & Aspartic acid protease & P. vulgaris & 2 & 32 & 0.04 & 6.44 & 56341 & protein \\
\hline 291 & Q8L994 & T-complex protein 1 subunit delta & A. thaliana & 3 & 45 & 0.04 & 7.68 & 58177 & protein \\
\hline 292 & A7XTY1 & Cytosolic glutathione reductase & P. vulgaris & 4 & 155 & 0.04 & 6.71 & 55127 & redox \\
\hline 293 & K4A852 & Uncharacterized protein & S. italica & 2 & 39 & 0.04 & 6.95 & 58150 & RNA \\
\hline 294 & F2DG93 & Predicted protein & H. vulgare & 4 & 149 & 0.03 & 6.62 & 64487 & amino acid metabolism \\
\hline 295 & D3U5B9 & Soluble acid invertase & S. bicolor & 9 & 148 & 0.03 & 5.63 & 62209 & major $\mathrm{CHO}$ metabolism \\
\hline 296 & K3ZS26 & Uncharacterized protein & S. italica & 7 & 104 & 0.03 & 8.02 & 60854 & mitoETC \\
\hline 297 & Q9SGR6 & AT1G76160 protein & A. thaliana & 4 & 84 & 0.03 & 8.4 & 60174 & not assigned \\
\hline 298 & Q1KUM7 & Putative uncharacterized protein & C. spinosa & 14 & 72 & 0.03 & 6.62 & 59525 & protein \\
\hline 299 & O81390 & Calcium-dependent protein kinase & N. tabacum & 5 & 53 & 0.03 & 7.03 & 61894 & signalling \\
\hline 300 & Q4W5U7 & Calnexin-like protein & S. lycopersicum & 5 & 73 & 0.03 & 4.82 & 61338 & signalling \\
\hline 301 & Q9ZWJ4 & Malic enzyme & A. arborescens & 9 & 135 & 0.03 & 6.44 & 64854 & TCA \\
\hline 302 & Q945U3 & Acyl-coenzyme A oxidase & G. $\max$ & 8 & 76 & 0.03 & 8.1 & 75115 & lipid metabolism \\
\hline 303 & B6SVK5 & ACS-like protein & Z. mays & 4 & 135 & 0.03 & 6.76 & 72915 & lipid metabolism \\
\hline 304 & D2XNF3 & Chaperone protein DnaK & P.patens & 11 & 254 & 0.03 & 5.5 & 75674 & stress \\
\hline 305 & F2DIS4 & Malic enzyme & H. vulgare & 6 & 109 & 0.03 & 7.4 & 68411 & TCA \\
\hline 306 & A5AJ44 & Putative uncharacterized protein & V. vinifera & 5 & 112 & 0.03 & 9.29 & 78977 & protein \\
\hline 307 & Q8GZR6 & Hydroxymethylbutenyl 4-diphosphate synthase & S. lycopersicum & 6 & 106 & 0.02 & 6.11 & 82805 & secondary metabolism \\
\hline 308 & A8IXU7 & Phototropin & C. reinhardtii & 7 & 36 & 0.02 & 8.47 & 81928 & signalling \\
\hline 309 & K3XDS7 & Uncharacterized protein & S. italica & 12 & 137 & 0.02 & 7.83 & 163179 & transport \\
\hline 310 & Q8VWE9 & Phospholipase D & P. somniferum & 7 & 63 & 0.02 & 5.85 & 92326 & lipid metabolism \\
\hline 311 & E5GC29 & Trehalose-6-phosphate synthase & C. melo & 5 & 95 & 0.02 & 5.76 & 95059 & minor $\mathrm{CHO}$ metabolism \\
\hline 312 & K4ALA1 & Uncharacterized protein & S. italica & 8 & 46 & 0.02 & 5.52 & 129179 & redox \\
\hline 313 & A8JGS8 & Beta'-cop & C. reinhardtii & 4 & 36 & 0.02 & 4.68 & 108352 & cell \\
\hline 314 & K3XV56 & Uncharacterized protein & S. italica & 7 & 70 & 0.02 & 4.97 & 103520 & cell \\
\hline 315 & Q9SQH4 & Actin bundling protein $\mathrm{ABP} 135$ & L. longiflorum & 6 & 52 & 0.02 & 5.97 & 107053 & cell \\
\hline 316 & B9HJ23 & Predicted protein & P. trichocarpa & 8 & 40 & 0.02 & 6.54 & 101700 & misc \\
\hline 317 & E5GBL8 & ATP-dependent clp protease & C. melo & 8 & 252 & 0.02 & 7.23 & 103416 & protein \\
\hline 318 & F2CYQ7 & Predicted protein & H. vulgare & 11 & 76 & 0.02 & 6.49 & 123962 & protein \\
\hline 319 & G3LY22 & Auto-inhibited Ca2+-transporting ATPase 10 & S. lycopersicum & 7 & 44 & 0.02 & 6.84 & 118705 & signalling \\
\hline
\end{tabular}




\begin{tabular}{|c|c|c|c|c|c|c|c|c|c|}
\hline 320 & A8HRR9 & Alpha-COP & C. reinhardtii & 5 & 47 & 0.02 & 7.31 & 139241 & cell \\
\hline 321 & K3YPB4 & Uncharacterized protein & S. italica & 5 & 180 & 0.01 & 6.4 & 149094 & protein \\
\hline 322 & Q9SUC7 & Tripeptidyl-peptidase 2 & A. thaliana & 10 & 143 & 0.01 & 6.46 & 155105 & protein \\
\hline
\end{tabular}

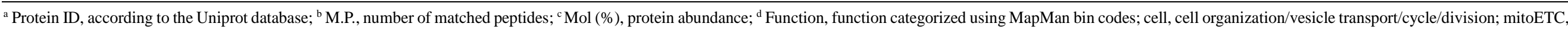

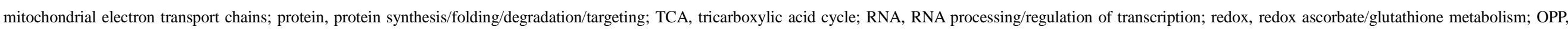
oxidative pentose phosphate; and misc, miscellaneous. 